University of Tennessee Health Science Center UTHSC Digital Commons

\title{
The Characterization of Hematopoiesis in Murine Fetal Bone
}

\section{Marrow}

Trent Daniel Hall

University of Tennessee Health Science Center

Follow this and additional works at: https://dc.uthsc.edu/dissertations

Part of the Medical Cell Biology Commons

\section{Recommended Citation}

Hall, Trent Daniel (https://orcid.org/0000-0003-0197-4328), "The Characterization of Hematopoiesis in Murine Fetal Bone Marrow" (2020). Theses and Dissertations (ETD). Paper 540. http://dx.doi.org/ 10.21007/etd.cghs.2020.0517.

This Dissertation is brought to you for free and open access by the College of Graduate Health Sciences at UTHSC Digital Commons. It has been accepted for inclusion in Theses and Dissertations (ETD) by an authorized administrator of UTHSC Digital Commons. For more information, please contact jwelch30@uthsc.edu. 


\title{
The Characterization of Hematopoiesis in Murine Fetal Bone Marrow
}

\begin{abstract}
Hematopoietic stem cells (HSCs) and their downstream progenitors are a heterogeneous population of cells that are indispensable for lifelong hematopoiesis and are often utilized in the clinic for the treatment of hematologic maladies via hematopoietic stem cell transplantation (HSCT). Over several decades, it has been discovered that HSCs arise in the dorsal aorta of the developing embryo, migrate to the fetal liver $(\mathrm{FL})$, and undergo a large expansion before reaching their final resting place in the bone marrow (BM). Many resources have been invested in understanding the roles of the different niches HSCs encounter along their journey. A greater understanding of HSC niche regulation could provide clues for HSC maintenance and expansion in vitro. One critical niche during HSC ontogeny that has been greatly overlooked is the fetal BM (FBM), of which the hematopoietic and HSC niche compartments are poorly understood. For this reason, we meticulously characterized the hematopoietic progenitor compartment of the whole skeleton FBM from its colonization until after birth via competitive transplantation, immunophenotypic analysis of the hematopoietic stem and progenitor cell (HSPC) compartment, functional assessment of specific progenitor populations, and single-cell RNA-sequencing (scRNA-Seq) of the hematopoietic and stromal FBM environment. Here, we provide the first report of the presence of bona fide HSCs within the E15.5 FBM. We also found that HSCs were present in the all sources of BM, including the forelimbs, hindlimbs, and trunk of E15.5 embryos. We are also the first to assess the BM immunophenotypic HSPC compartment from initial seeding to adulthood and found that specific multipotent progenitor (MPP) cells (MPP2s) are the predominant HSPC population in the FBM, appearing to have the ability to migrate to and seed the FBM directly from the FL. Interestingly, immunophenotypic MPP2s are not functional in vitro or in vivo until birth (E18.5-P0), and display reduced repopulating capacity compared to adult BM and time-matched FL MPP2s. Also, the frequencies of the different FBM HSPCs shift around birth from an MPP2-dominant phenotype to the MPP3/MPP4-dominant phenotype seen in adult BM. To identify the intrinsic and extrinsic mechanisms controlling MPP2 functional maturation, we isolated stromal and hematopoietic progenitor (HP) populations from E16.5, E18.5, P0, and adult BM, and constructed the first known scRNA-Seq dataset spanning the HP and stromal compartment across BM ontogeny. Preliminary analysis of our scRNA-Seq datasets show that the FBM stroma and HP compartments are compositionally distinct from the adult BM compartments, and this disparity in composition is even more drastic at E16.5, suggesting that the reduced function of FBM MPP2s may be due to a semi-incompatible FBM niche. Our future studies will focus on identifying intrinsic differences between immunophenotypic HSPC populations across FBM ontogeny in our HP scRNA-Seq dataset, as well as defining putative niches for HSPCs in the FBM. We hope that these analyses will identify novel hematopoietic factors in the FBM niche that can be therapeutically exploited to enhance HSC expansion/function/differentiation in the clinic.
\end{abstract}

Document Type

Dissertation

Degree Name

Doctor of Philosophy (PhD)

Program

Biomedical Sciences

Research Advisor

Shannon McKinney-Freeman, PhD 


\section{Keywords}

Bone Marrow, Cell Biology, Development, Hematopoiesis, Mouse Models, Stem Cells

\section{Subject Categories}

Medical Cell Biology | Medical Sciences | Medicine and Health Sciences 


\section{The Characterization of Hematopoiesis in Murine Fetal Bone Marrow}

Author:

Trent Daniel Hall
Advisor: Shannon McKinney-Freeman, $\mathrm{PhD}$

A Dissertation Presented for The Graduate Studies Council of

The University of Tennessee Health Science Center in Partial Fulfillment of the Requirements for the Doctor of Philosophy degree from

The University of Tennessee

in

Biomedical Sciences: Cancer and Developmental Biology College of Graduate Health Sciences 
Copyright $(\subset) 2020$ by Trent Hall. All rights reserved. 


\section{DEDICATION}

This work is dedicated to my wife and our children. Emily, thank you for your unending patience and love. I would be utterly lost without you. Kids, I hope you will

find a small sliver of inspiration here when you are older and life gets too busy. I love you all so much! 


\section{ACKNOWLEDGEMENTS}

I would first like to thank my mentor, Dr. Shannon McKinney-Freeman. I am forever indebted to her for the rigorous, persistent, patient, and fun training she provided for me over the years. No one is a greater advocate for their trainees' intellectual and professional development than Dr. McKinney-Freeman, and I will always look to her as a model for my conduct as a scientist, mentor, and colleague.

I would also like to thank the members of my committee: Dr. John Cox, Dr. Eric Enemark, Dr. Gerard Grosveld, Dr. Susan Miranda, Dr. Martine Roussel, and Dr. Mitch Weiss. Our discussions were vital to the progression of this story and to my development as a scientist. Thank you for agreeing to lead me through this process and for your desire to help me succeed.

Thank you to UTHSC, the CGHS, and the Cancer and Developmental Biology program leadership, faculty, and staff for giving me the opportunity to pursue my $\mathrm{PhD}$. Also, I would like to thank St. Jude Children's Research Hospital, with specific attention to the faculty and staff of the Department of Hematology, the Flow Cytometry Core, and the Animal Resource Center, without which none of this work would be possible.

I give my sincerest thanks to all the McKinney-Freeman lab mates I have had the pleasure of working with over the past seven years. Our conversations (scientific or otherwise) have been stimulating and fun, and I could not have asked for better colleagues. I would like to especially thank Dr. Miguel Ganuza, who was a tremendous mentor to me upon my entry into the lab. Dr. Ganuza's bottomless curiosity and unwavering rigor as a scientist still inspires me to think creatively when designing experiments and to strive for perfection at the bench, and he will always be a valued friend.

Finally, I would like to thank my wife, our parents, and our children for their constant encouragement. I love you all!

This work was funded in part by the NIDDK (F31DK118798). 


\begin{abstract}
Hematopoietic stem cells (HSCs) and their downstream progenitors are a heterogeneous population of cells that are indispensable for lifelong hematopoiesis and are often utilized in the clinic for the treatment of hematologic maladies via hematopoietic stem cell transplantation (HSCT). Over several decades, it has been discovered that HSCs arise in the dorsal aorta of the developing embryo, migrate to the fetal liver (FL), and undergo a large expansion before reaching their final destination in the bone marrow (BM). Many resources have been invested in understanding the roles of the different niches HSCs encounter along their journey. A greater understanding of HSC niche regulation could provide clues for HSC maintenance and expansion in vitro. One critical niche during HSC ontogeny that has been greatly overlooked is the fetal BM (FBM), of which the hematopoietic and HSC niche compartments are poorly understood. For this reason, we meticulously characterized the hematopoietic progenitor compartment of the whole skeleton FBM from its colonization until after birth via competitive transplantation, immunophenotypic analysis of the hematopoietic stem and progenitor cell (HSPC) compartment, functional assessment of specific progenitor populations, and single-cell RNA-sequencing (scRNA-Seq) of the hematopoietic and stromal FBM environment. Here, we provide the first report of the presence of bona fide HSCs within the E15.5 FBM. We also found that HSCs were present in all sources of BM, including the forelimbs, hindlimbs, and trunk of E15.5 embryos. We are also the first to assess the BM immunophenotypic HSPC compartment from initial seeding to adulthood and found that specific multipotent progenitor (MPP) cells (MPP2s) are the predominant HSPC population in the FBM, appearing to have the ability to migrate to and seed the FBM directly from the FL. Interestingly, immunophenotypic MPP2s are not functional in vitro or in vivo until birth (E18.5-P0), and display reduced repopulating capacity compared to adult BM and time-matched FL MPP2s. Also, the frequencies of the different FBM HSPCs shift around birth from an MPP2-dominant phenotype to the MPP3/MPP4dominant phenotype seen in adult BM. To identify the intrinsic and extrinsic mechanisms controlling MPP2 functional maturation, we isolated stromal and hematopoietic progenitor (HP) populations from E16.5, E18.5, P0, and adult BM, and constructed the first known scRNA-Seq dataset spanning the HP and stromal compartment across BM ontogeny. Preliminary analysis of our scRNA-Seq datasets show that the FBM stroma and HP compartments are compositionally distinct from the adult BM compartments, and this disparity in composition is even more drastic at E16.5, suggesting that the reduced function of FBM MPP2s may be due to a semi-incompatible FBM niche. Our future studies will focus on identifying intrinsic differences between immunophenotypic HSPC populations across FBM ontogeny in our HP scRNA-Seq dataset, as well as defining putative niches for HSPCs in the FBM. We hope that these analyses will identify novel hematopoietic factors in the FBM niche that can be therapeutically exploited to enhance HSC expansion/function/differentiation in the clinic.
\end{abstract}




\section{TABLE OF CONTENTS}

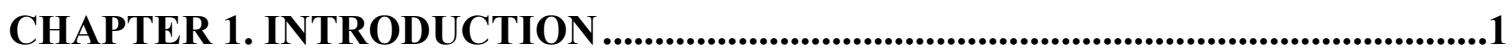

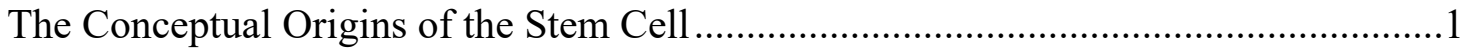

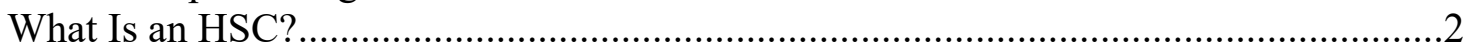

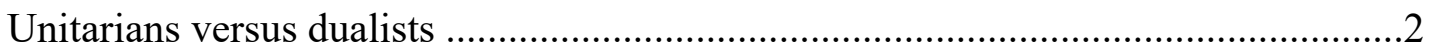

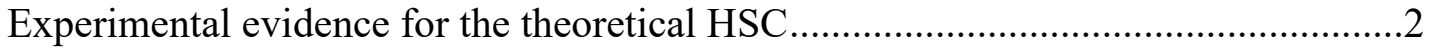

Development of assays for identifying properties of HSCs................................6

HSC isolation and establishment of a hematopoietic hierarchy ..............................8

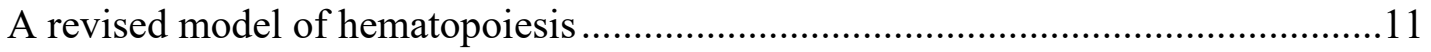

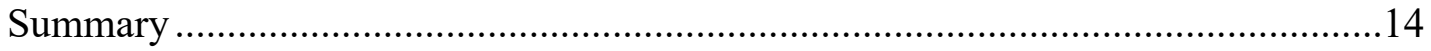

Where Do Hematopoietic Cells Come From? ..........................................................14

The first primitive wave is established by the hemangioblast .................................16

The first primitive wave: Blood cell types and their fate........................................17

The second primitive wave: Multipotent progenitors that are distinct from

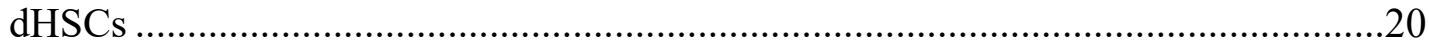

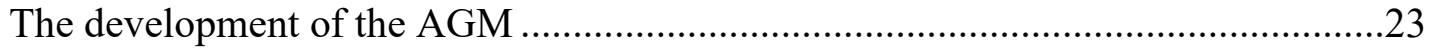

The AGM is the sole source of dHSC formation...................................................24

How does the AGM generate dHSCs? .............................................................25

Is the dHSC the foundational hematopoietic cell for steady state hematopoiesis?....29

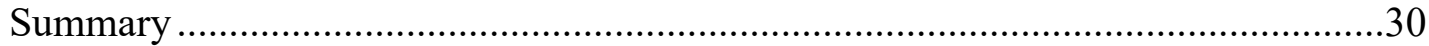

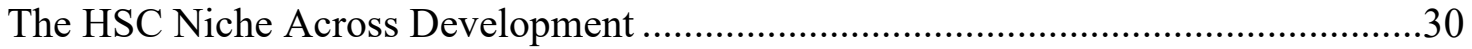

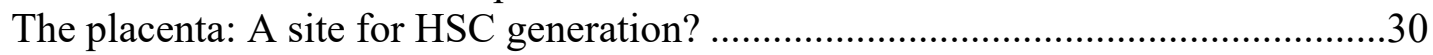

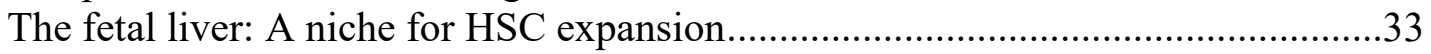

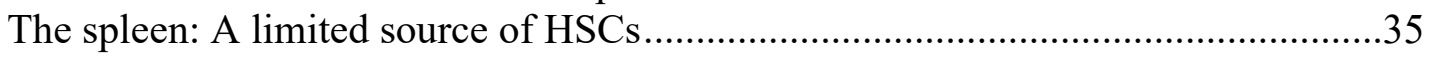

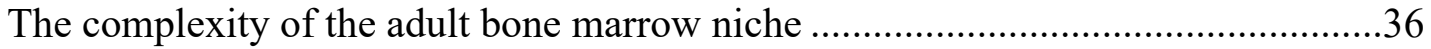

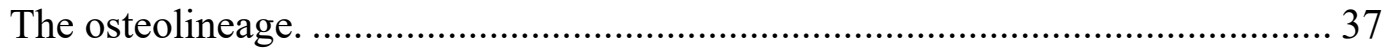

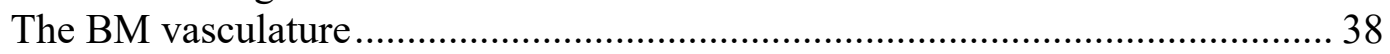

The sympathetic nervous system: Non-myelinating Schwann cells................... 39

Perivascular CAR cells ............................................................................... 39

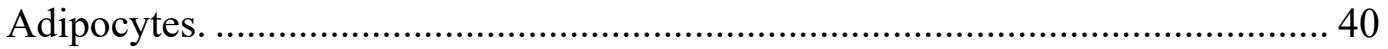

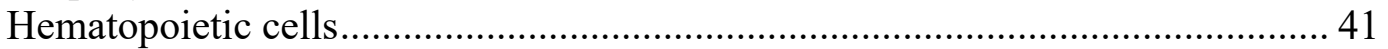

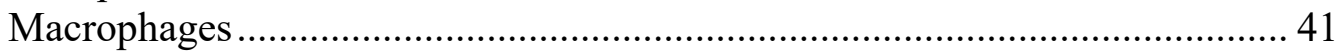

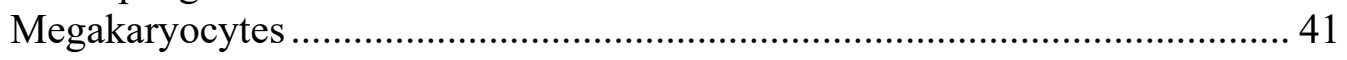

Other hematopoietic cells ............................................................... 42

The fetal bone marrow: An understudied but crucial niche in HSC ontogeny ..........42

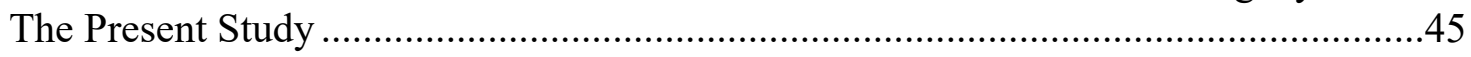

CHAPTER 2. METHODS ..........................................................................................47

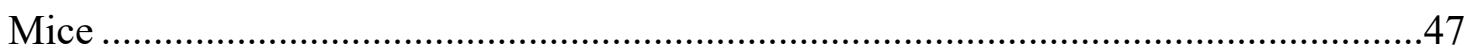

Isolation of Hematopoietic and Stromal Cells ......................................................47

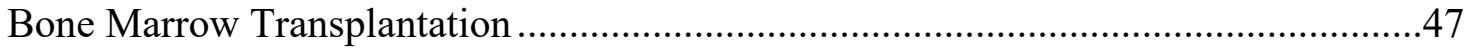

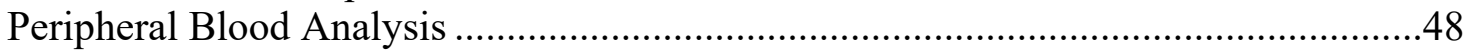

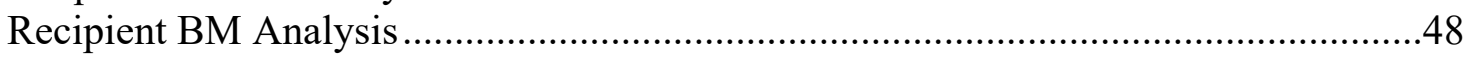


Analysis of Hematopoietic Stem and Progenitors Across Bone Marrow

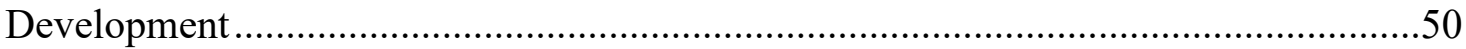

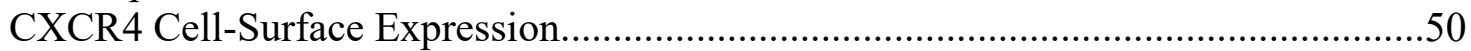

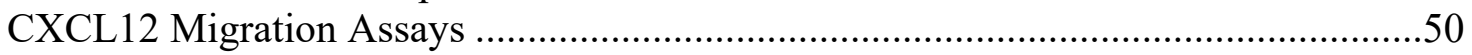

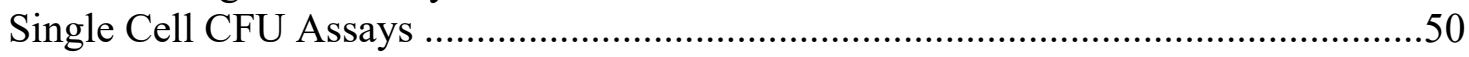

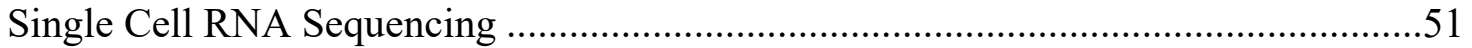

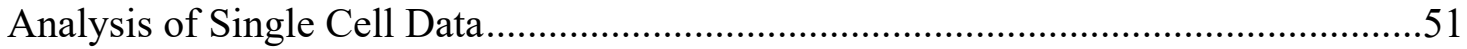

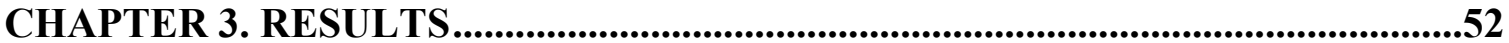

Long-Term Repopulating Cells Can Be Found in E15.5 FBM .................................52

Bona Fide HSCs Are Present in the E15.5 FBM.......................................................59

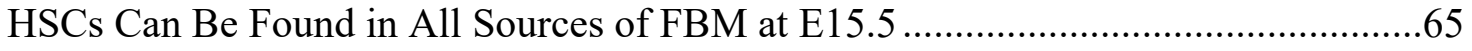

The FBM HSPC Compartment Is Compositionally Distinct From FL and Adult

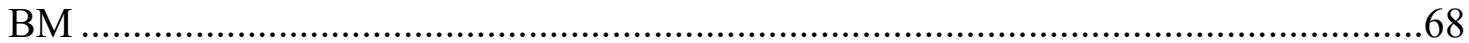

All FL HSPCs Have the Potential to Migrate to the FBM ..........................................71

FBM MPP2s Are Functionally Distinct From FL and Adult MPP2s..........................72

The FBM Hematopoietic Progenitor Compartment Undergoes Drastic Remodeling

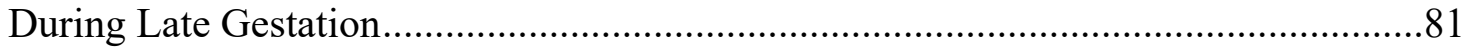

Immunophenotypic MPP2s Display Highly Heterogeneous Transcriptional

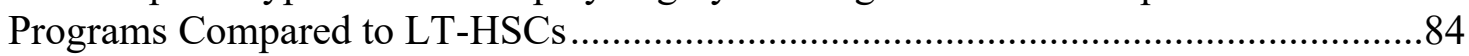

The Stem Cell-Specific Transcriptional Profiles of Immunophenotypic LT-HSCs

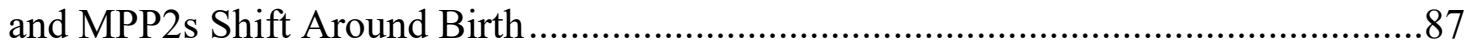

The FBM Stromal Compartment Contains Putative Niche Cells for HSPCs ...............90

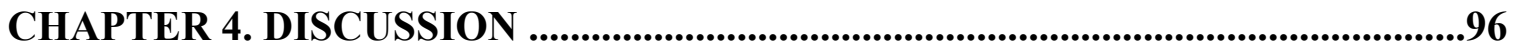

The Presence of Bona Fide HSCs in the E15.5 FBM...............................................96

Potential Mechanisms for the Shift in HSPC Frequencies Around Birth.....................99

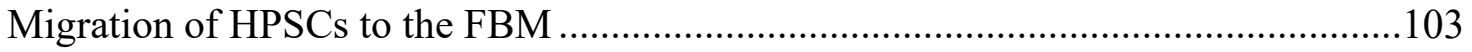

Functional Heterogeneity and Maturation of BM HSPCs .................................... 105

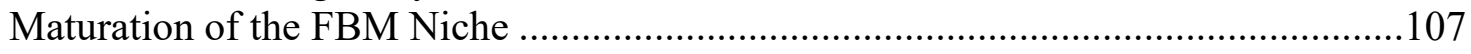

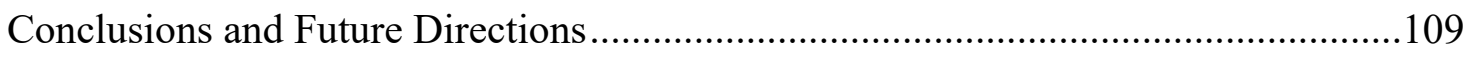

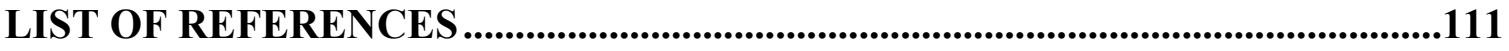

VITA 


\section{LIST OF TABLES}

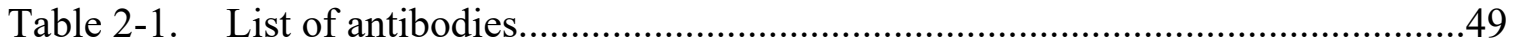




\section{LIST OF FIGURES}

Figure 1-1. Till and McCulloch identify clonal hematopoietic progenitors .....................5

Figure 1-2. The classic model of hematopoiesis ................................................ 10

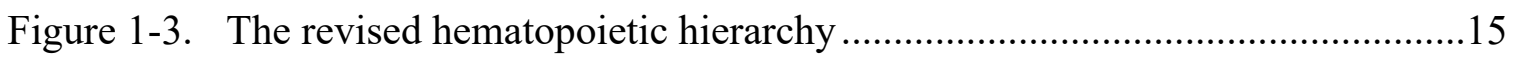

Figure 1-4. The first primitive wave of hematopoiesis.......................................... 18

Figure 1-5. The second primitive wave of hematopoiesis ........................................22

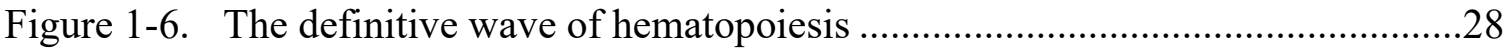

Figure 1-7. Developmental ontogeny of definitive hematopoiesis..............................31

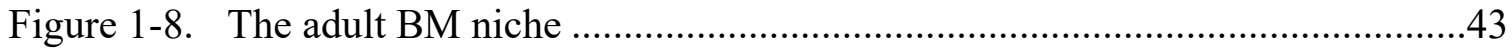

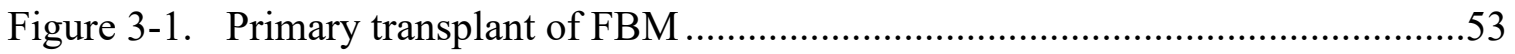

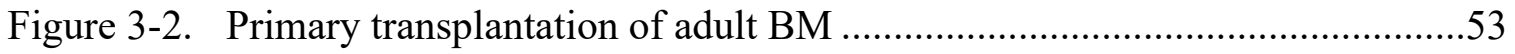

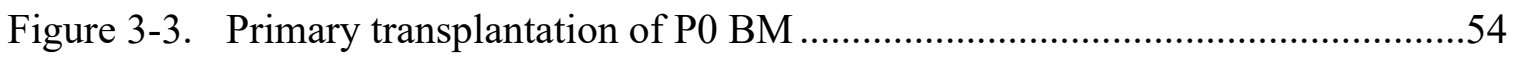

Figure 3-4. Primary transplantation of E18.5 FBM...............................................56

Figure 3-5. Primary transplantation of E17.5 FBM...............................................56

Figure 3-6. Primary transplantation of E16.5 FBM..................................................57

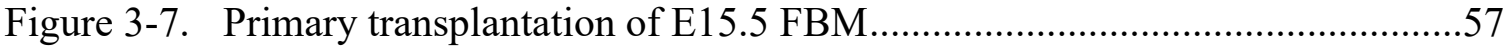

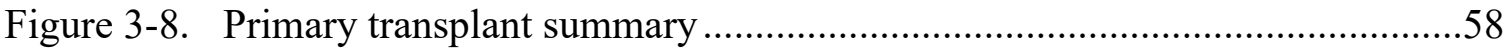

Figure 3-9. Secondary transplant of FBM .........................................................60

Figure 3-10. Secondary transplantation of adult BM ...............................................61

Figure 3-11. Secondary transplantation of P0 BM .................................................61

Figure $3-12$. Secondary transplantation of E18.5 FBM...........................................62

Figure 3-13. Secondary transplantation of E17.5 FBM..........................................62

Figure 3-14. Secondary transplantation of E16.5 FBM..............................................64

Figure 3-15. Secondary transplantation of E15.5 FBM.........................................64

Figure 3-16. Transplantation of individual E15.5 fetal bones .....................................66 
Figure 3-17. HSCs are found in all E15.5 BM sources ...........................................67

Figure 3-18. Analysis of HSPCs across BM and liver ontogeny ................................69

Figure 3-19. Frequency and total number of HSPCs in BM and liver ...........................70

Figure 3-20. FL HSPC CXCR4 cell surface expression..........................................73

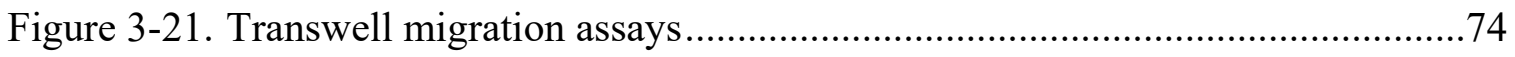

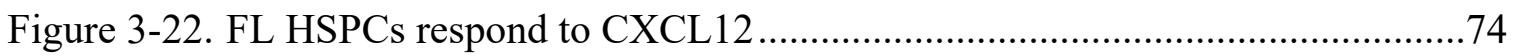

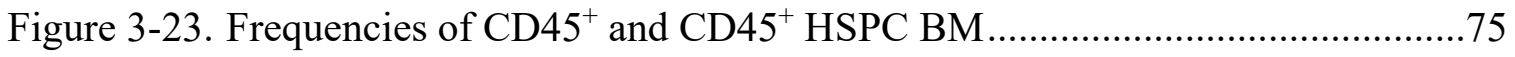

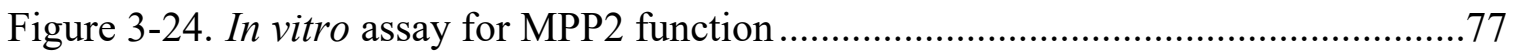

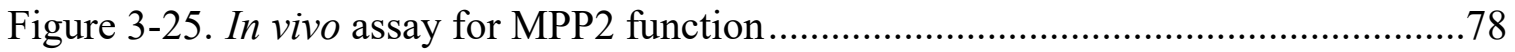

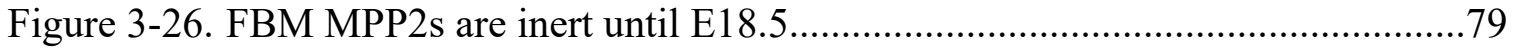

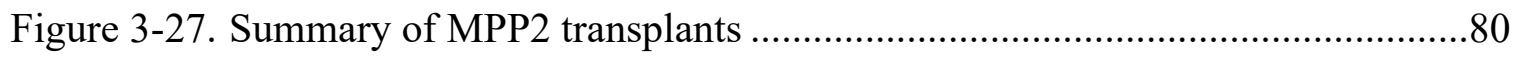

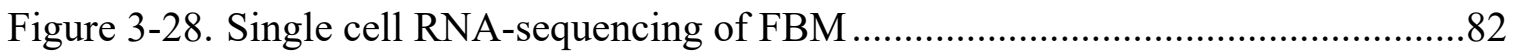

Figure 3-29. Hematopoietic clusters in FBM scRNA-Seq data ..................................83

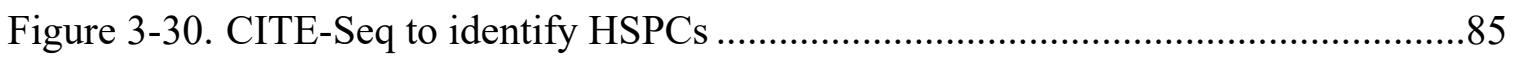

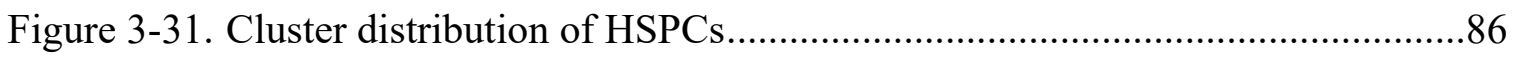

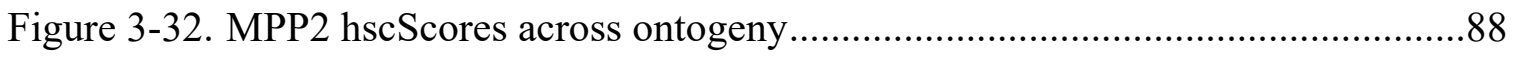

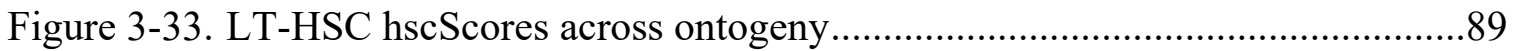

Figure 3-34. Stromal clusters in FBM scRNA-Seq data .......................................... 91

Figure 3-35. Stomal Cxcl12 expression across BM ontogeny ....................................93

Figure 3-36. Stromal Kitl expression across BM ontogeny.......................................94

Figure 4-1. A revised developmental timeline of FBM hematopoiesis........................97

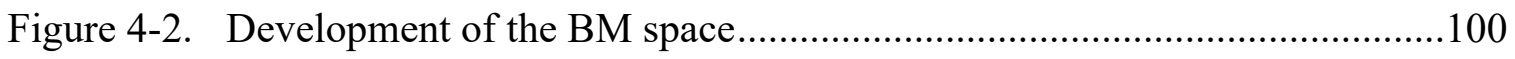

Figure 4-3. Potential effectors of HSPC frequency at birth...................................... 101

Figure 4-4. Models for HSPC migration and expansion in the FBM ........................... 104

Figure 4-5. Hypotheses for FBM MPP2 functional maturation .................................106 


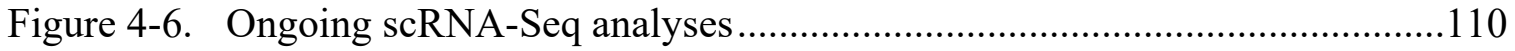




\section{LIST OF ABBREVIATIONS}

AGM

BM

CAR

CFU

CFU-C

CFU-S

CITE-Seq

CLP

CMP

CRU

DA

dHSC

e.e

E15.5

EMP

EPO

ESC

FACS

FBM

FITC

FL

Flt3L

G-CSF

GM

GM-CSF

GMP

HP

HPP-CFC

HSC

HSCT

HSPC

HUVEC

iPSC

IT-HSC

LMPP

LSK

LTC-IC

LT-HSC

MEP

MPP

msHSC

$\mathrm{P} 0$

PB
Aorta Gonad Mesonephros

Bone Marrow

CXCL12-Abundant Reticulocyte

Colony Forming Unit

Colony Forming Unit-Cell

Colony Forming Unit-Spleen

Cellular Indexing of Transcriptomes and Epitopes by Sequencing

Common Lymphoid Progenitor

Common Myeloid Progenitor

Competitive Repopulating Unit

Dorsal Aorta

Definitive Hematopoietic Stem Cell

Embryo Equivalent

Embryonic Day 15.5

Erythromyeloid Progenitor

Erythropoietin

Embryonic Stem Cell

Fluorescence-Activated Cell Sorting

Fetal Bone Marrow

Fluorescin Isothiocyanate

Fetal Liver

Flt3-Ligand

Granulocyte Colony-Stimulating Factor

Granulocyte/Macrophage

Granulocyte-Macrophage Colony-Stimulating Factor

Granulocyte/Macrophage Progenitor

Hematopoietic Progenitor

High-Proliferative Potential Colony Forming Cell

Hematopoietic Stem Cell

Hematopoietic Stem Cell Transplant

Hematopoietic Stem and Progenitor Cell

Human Umbilical Vein Endothelial Cell

Induced Pluripotent Stem Cell

Long-Term Hematopoietic Stem Cell

Lymphoid-Primed Multipotent Progenitor

Lineage $-\mathrm{Sca}-1^{+} \mathrm{c}-\mathrm{Kit}^{+} \mathrm{Cell}$

Long-Term Culture-Initiating Cell

Long-Term Hematopoietic Stem Cell

Megakaryocyte/Erythroid Progenitor

Multipotent Progenitor

Muscle Hematopoietic Stem Cell

Postnatal Day 0

Peripheral Blood 


$\begin{array}{ll}\text { PBS } & \text { Phosphate Buffered Saline } \\ \text { P-Sp } & \text { Para-Aortic Splanchnoleura } \\ \text { RBC } & \text { Red Blood Cell } \\ \text { RU } & \text { Repopulating Unit } \\ \text { SCF } & \text { Stem Cell Factor } \\ \text { scRNA-Seq } & \text { Single-Cell RNA Sequencing } \\ \text { SP } & \text { Side Population } \\ \text { ST-HSC } & \text { Short-Term Hematopoietic Stem Cell } \\ \text { TPO } & \text { Thrombopoietin } \\ \text { T-reg } & \text { Regulatory T-Cell } \\ \text { UGR } & \text { Urogenital Ridges } \\ \text { WBM } & \text { Whole Bone Marrow } \\ \text { YS } & \text { Yolk Sac } \\ \text { 5-FU } & \text { 5-Fluorouracil }\end{array}$




\section{CHAPTER 1. INTRODUCTION}

\section{The Conceptual Origins of the Stem Cell}

It was the German zoologist Ernst Haeckel who, after reading and being heavily influenced by Charles Darwin's work, began drawing Stammbäume - or "stem trees" - to describe the phylogeny of all living organisms (Haeckel 1868, Haeckel 1874). This led him to describe the unicellular organism from which he presumed all multicellular organisms derived as a "Stammzelle," or "stem cell" (Haeckel 1868, Haeckel 1874). Haeckel, also a developmental biologist, simultaneously used the term to refer to the fertilized egg of multicellular organisms (Haeckel 1877).

In 1885, August Weismann theorized that germ-plasm, a substance that is passed down through generations via sexual reproduction, is transmitted via specialized "germ cells" that are distinct from the rest of the body (Weismann 1885). This led the German biologist Theodor Boveri to try to identify the earliest germ cells in nematodes via microscopy. By tracing the cell lineages of fertilized Ascaris embryos in tree diagrams (again, coined "Stammbäume"), Boveri noticed that the germline cells that were destined to give rise to "somatic" cells displayed fragmentation and loss of chromatin as the cells divided. However, the germline cells destined to give rise to germ cells appeared to maintain the same compliment of chromatin along lineage commitment. Essentially, Boveri used this observation to conclude that these cells maintained the chromatin so that they could pass it on to the next generation. He called the cells that existed between the fertilized cell to the fully committed germ cells, "stem cells" (Boveri 1887, 1892a, 1892b). Around the same time, Häcker identified a cell in Cyclops embryos that become internalized upon gastrulation and underwent asymmetric cell division to produce a mesodermal precursor and a germ cell precursor and called it a "stem cell" (Häcker 1892). However, Häcker also used the term for cells that produce oocytes in the gonad later in development (Häcker 1892).

It was not until 1896 that the term "stem cell" became popularized in the English language by Edmund Wilson's "The Cell in Development and Inheritance," in which he reviewed Boveri's and Häcker's works (Wilson 1896). However, the conceptual definition of the "stem cell" was still geared toward a description of a cell that facilitates the production of germ cells.

In this introduction, I will review the origins and current state of hematopoietic stem cell (HSC) research. HSCs are the most well-characterized stem cell population to date, and their properties are often used as a standard for identifying stem cell populations of other tissues. The discovery of HSCs and their properties have also led to their widespread clinical use in hematopoietic stem cell transplantation (HSCT). Here, I will discuss the discovery and characterization of HSCs, the developmental origins of HSCs, and the regulatory niches that HSCs interact with during development and steady state. Finally, I will introduce the primary study central to this dissertation: characterizing the establishment of hematopoiesis in the fetal bone marrow (BM) 


\section{What Is an HSC?}

\section{Unitarians versus dualists}

Once Paul Ehrlich established staining techniques for leukocytes using alkaline, acid, and neutral dyes (Ehrlich, 1879), different white blood cell lineages could be observed. This led to years of research in which hematologists were divided as to the origins of different blood cell lineages (Maximow, 1924). Unitarians believed there was a common cell of origin for granulocytes, erythrocytes, and lymphocytes. Meanwhile, dualists believed that myeloid cells arose from common precursors in the BM and lymphoid cells derived from common precursors in the lymphatic glands/spleen. This made sense at the time, because the lymphatic system was the first tissue believed to harbor hematopoietic activity (Müller, 1844), while the BM was thought to be the primary site of hematopoiesis in the adult (Neumann, 1868). Therefore, many dualists (including Ehrlich) believed that different committed cells could be coming from distinct sources of hematopoietic tissue (Cooper, 2011).

Based on Ehrlich's staining protocols, it was clear that lymphocytes differed from other leukocytes and erythrocytes phenotypically. It was also noted that lymphocytes resembled more immature cells (e.g. the progenitor cells), therefore the first names given by the unitarians for a putative hematopoietic cell of origin took on lymphocytic nomenclatures [e.g., nongranular undifferentiated lymphocytes (Dantschakoff, 1908); lymphoidocytes (Pappenheim 1908)], although other terms were given [e.g. primary wandering cells (Saxer, 1896); hemocytoblasts (Zoja, 1910)].

Alexander Maximow is typically credited with coining the term "stem cell" to refer to the common hematopoietic cell of origin (1909), although it had been used as early as 1896 by Pappenheim (Pappenheim 1896) to describe a precursor cell capable of giving rise to both red and white blood cells. Neumann-who first hypothesized that the BM is the primary source of hematopoiesis in adults - also used the term "stem cell" to describe such a cell (Neumann, 1912), and he and others thought that the only way to determine if the unitarians or dualists were correct was to somehow assay the potential of the "stem cells" in in vitro culture. Therefore, at this time the term "stem cell" was used to at least describe both 1.) the cell of origin for germ cells and 2.) the common cell of origin for hematopoietic cells.

\section{Experimental evidence for the theoretical HSC}

Until the 1960s, there was no experimental evidence of a clone with HSC properties, namely, the ability to self-renewal and differentiate into all blood lineages. From 1945-1961, several studies focusing on transplantation led to the unequivocal discovery of HSCs. In 1945, Ray Owen looked for the presence of inherited cellular antigens in the blood of fraternal twin calves and found that the majority of twins have identical blood types, meaning that one twin had a portion of cells similar to the other 
twin and vice versa (Owen 1945). Owen postulated that since the only time there was interchange of blood was during embryogenesis, there must be some sort of cell from one twin - the "donor" - that establishes itself as a source of hematopoietic tissue in the other twin - the "host" - and continues to produce blood cells throughout adulthood (Owen 1945).

At the same time, the atomic bombings of 1945 in Japan shed light on the effects of total body irradiation on the hematopoietic system, as many civilians died of hematopoietic failure after being exposed. Therefore, many researchers focused on methods to shield the body from the effects of irradiation on the hematopoietic system. In 1950, Jacobson found that total body irradiation (600 rads) incurs severe anemia in mice, mostly as an effect of BM destruction. However, this could be ameliorated by a compensatory mechanism from the spleen, as mice that are given irradiation after splenectomy followed by reintroduction of the spleen show recovery of anemia after a recovery of BM hematopoiesis (Jacobson 1950). Irradiating the spleens prevents this compensatory mechanism from happening, but shielding the spleens with $1 / 4$ inch lead prevents the spleens from becoming compromised by the irradiation (Jacobson 1950). Lorenz showed that injection of heterologous and homologous BM into lethally irradiated mice and guinea pigs allowed for a recovery from radiation-induced death in recipients (Lorenz 1951). It was also noted that intravenous injections of homologous BM produced a greater incidence of survival (Lorenz 1951). Lorenz speculated that the factor providing protection from mortality was probably humoral, but he did not rule out the possibility of a cellular factor (Lorenz 1951).

In another study of cattle twins, Billingham tested whether skin grafts of twins from heterozygous and monozygotic twins would be accepted, with the assumption that monozygotic twins would perform better (Billingham 1952). However, they found that the grafts were accepted regardless of zygosity. They relied on the work of Owen to speculate that this acceptance was due to the "confluence of foetal circulations of cattle twins" (Billingham 1952). One year later, Billingham proposed the hypothesis of "acquired tolerance," by injecting fetal mice/chicks with cells from a different strain, followed by skin graft in adulthood. They showed that the creation of chimeras in the prenatal period allowed for immunological tolerance to skin grafts in adulthood from the same strain they were inoculated against (Billingham 1953). This led Main in 1955 to inject lethally irradiated mice of one strain with the BM of a different strain, followed by an attempted skin graft from the donor strain to see if the creation of a BM chimera would facilitate a successful skin graft (Main 1955). They were successfully grafted, thus suggesting the creation of murine "tolerant chimeras." However, there was still no confirmation of a humoral or cellular effect that allowed for tolerance of the graft (Main 1955).

In two groundbreaking studies, Nowell and Ford confirmed that the source of radioprotection in mice after BM or spleen injection was of cellular origin, as they showed via chromosomal marker staining that the donor cells established in the recipient BM and showed continued hematopoietic output (Nowell 1956, Ford 1956). The same year, mice were transplanted with isologous (same genetic background), homologous 
(same species), or heterologous (different species) BM following lethal irradiation to confirm that isologous and homologous BM offered the greatest protection (Trentin 1956). Shortly thereafter, the first human BM transplant was performed by E. Donnall Thomas (Thomas 1957). Importantly, human transplants showed that donor BM was able to engraft following moderate doses of radiation, and engraftment seemed to be prolonged after a radiation dose strong enough to produce bone-marrow aplasia in the patient had been administered. While instrumental to driving HSCT forward, these studies still did not confirm the existence of an HSC.

It was not until the 1960s that Ernest MuCulloch and James Till provided the first evidence of a true HSC. In one of their first studies, McCulloch and Till irradiated murine $\mathrm{BM}$ in vivo or ex vivo, diluted the irradiated cells to a known concentration, and injected the cell suspensions into lethally irradiated recipients in a series of limiting dilutions (McCulloch 1960). To quantify the number of protected repopulating cells, they used the percentage of surviving mice as a readout (McCulloch 1960). They also tested the number of radioprotected repopulating cells in the BM by subjecting their cell suspensions (or cells in vivo) to various doses of irradiation (McCulloch 1960). This was a key step to uncovering a quantitative method for identifying HSCs.

One of the defining moments in discovering the potential HSC was the observation that $\sim 10$ days after injecting mice with unirradiated BM cells, the spleens of the recipients formed several distinct nodules (Till 1961). McCulloch and Till called the cells that produced these nodules Colony Forming Units, or "CFU" (subsequently referred to as CFU-S for "CFU-Spleen"). They could also use the CFU-S in recipients as a readout to quantify the number of CFU-S in the injected donor BM, providing another quantification method for potential HSCs (Till 1961). Since there was a positive correlation between the number of CFU-S in recipients and the number of marrow cells injected, the prevailing notion was that the CFU-S were arising from single clones.

In a landmark study, McCulloch and Till induced chromosomal aberrations in donor BM to produce putative chromosomal markers of clonality in the BM resident CFU-S, followed by injection of BM and an observation of the clonal markers in cell suspensions of the recipient CFU-S (Becker 1963) (Figure 1-1). They found that the CFU-S was indeed a single clone, as nearly $100 \%$ of cells in a colony presented identical chromosomal markers (Becker 1963). This was the first proof of a hematopoietic clone in mouse BM. McCulloch and Till then wanted to show that the CFU-S had the ability to self-renew, a critical property of stem cells. Therefore, they isolated the CFU-S from primary recipients and secondarily transplanted them into new recipients to see if new CFU-S could be formed (Siminovitch 1963). They found that in some secondary recipients, new CFU-S were formed, confirming that the CFU-S had the stem cell property of self-renewal.

For the remainder of the decade, other key properties of the modern-day HSC were described using CFU-S as a model. Becker tested cells from different sources for their presence in $\mathrm{S}$ phase via exposure to thymidine followed by assaying them for CFU$\mathrm{S}$ ability and found that a large proportion of CFU-S from FL and from proliferating 


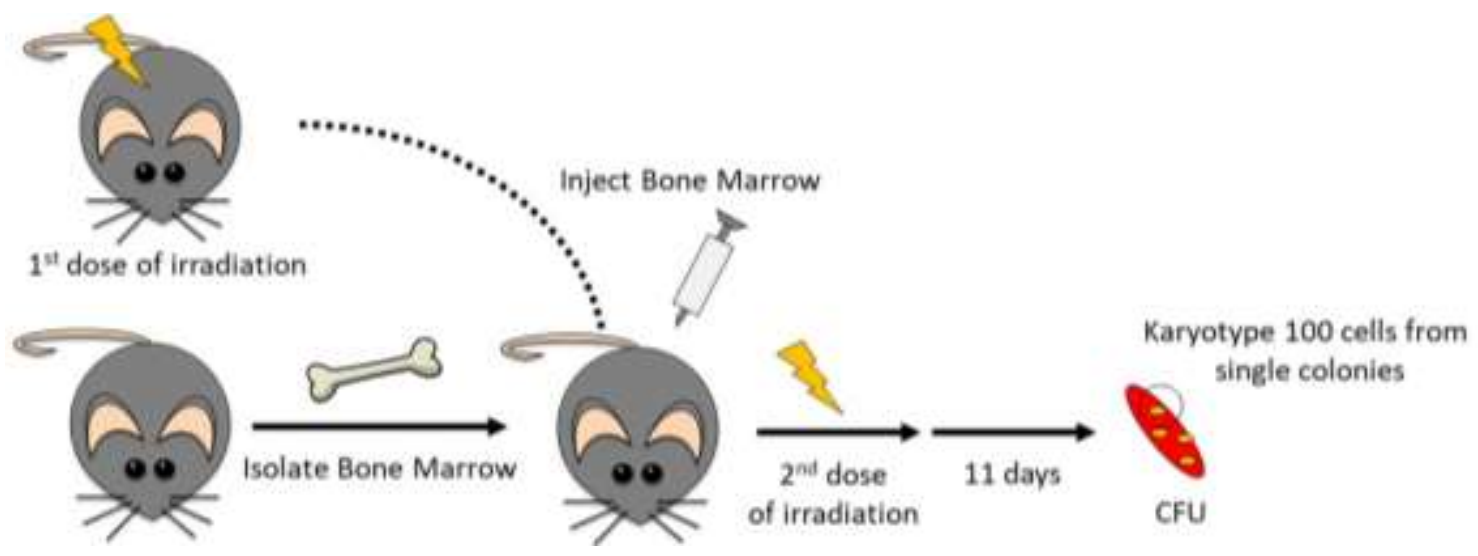

Figure 1-1. Till and McCulloch identify clonal hematopoietic progenitors

The experimental schematic used in Becker 1963 to identify the first hematopoietic clone in the mouse bone marrow. Injected bone marrow was irradiated within partially irradiated hosts to induce chromosomal abnormalities in donor cells, which could later be tracked for clonal contribution in CFU-S. 
transplants were in S phase, while adult spleen and BM CFU-S showed little to no cells in S phase (Becker 1965). This suggested that the CFU-S in adult BM was a quiescent population. In 1966, it was found that chemotherapeutic compounds most effective against cells undergoing division had less effect on the CFU-S ability of normal hematopoietic cells from mouse BM, suggesting that CFU-S are primarily in a "resting" or quiescent state (Bruce 1966). CFU-S were determined to be multipotent progenitors via the production of mature erythroid, megakaryocyte, granulocyte, and macrophage cell lineages in colonies (Wu 1967). Using the genetic labelling technique of Becker 1966, CFU-S were thought to have lymphoid potential via formation of lymphoid cells in the thymus of recipients of donor BM (Wu 1968a). This was the first demonstration of a progenitor with lymphoid, myeloid, and erythroid potential, although BM HSCs were likely the source of these donor lymphocytes.

While the CFU-S assay was critical for driving the search for the true HSC forward, it was eventually discovered and confirmed that the CFU-S was not the true HSC population. In 1979, the existence of a "pre-CFU-S" was hypothesized based on the observation that upon injecting 5-fluorouracil (5-FU) treated BM cells, there was an increase in the CFU-S potential of 5-FU recipient BM compared to untreated BM, and CFU-S showed a delay in formation in 5-FU treated BM recipients (Hodgson 1979). In a critical study, it was discovered that many of the CFU-S from 7-9 days post-transplant are not found 9-12 days post-transplant (Magli 1982). It was also found that only the 9-12 day CFU-S contain "primitive progenitors," while the 7-9 day CFU-S display an erythroid bias in in vitro colony forming assays (Magli 1982). It was therefore concluded that the notion that CFU-S are maturing and colonies are gaining more progenitors is likely not due to the nature of the clones to self-renew, but rather the appearance of new clones coming from some other source ( $\mathrm{Na} 2002)$.

In the late 70 s and early $80 \mathrm{~s}$, HSC transplantation was accelerating as a therapy for maladies of the hematopoietic system. Therefore, it was understood that HSCs were likely to exist and contribute to the regeneration of the hematopoietic system of transplant recipients, but there was broad agreement in the field that several progenitors contributed to regeneration. In turn, there was a shift in research aims from proving the existence of an HSC to isolating the HSC, with the defining features of HSCs-influenced by the incredible groundwork provided by McCulloch and Till and others - being single cells that display multipotency and self-renewal capacity (Weissman 2008).

\section{Development of assays for identifying properties of HSCs}

The ability to test for hematopoietic multipotency and self-renewal capacity was heavily supported by the development of protocols to assay these properties from different cell types via in vitro culture. These assays also paved the way for conceptualizing the presence of intermediate progenitors between the HSC and mature blood lineages. The primary limitation was that these assays only allowed for detection of myeloid output. In 1965, Pluznik plated spleen cells at limiting dilutions on agar with embryonic "feeder" cells and found that there was a positive correlation with the number 
of cells plated and the number of mast cell colonies formed (Pluznik 1965). This helped established an in vitro clonal assay for hematopoietic cells of the granulocyte/macrophage (GM) lineages (CFU-C). This was later replicated for mouse (Bradley 1966) and human (Senn 1967) BM. Later, CFU-S cells were plated in the same assay and CFU-C were observed, suggesting that there was a close relationship between the two types of CFU (Wu 1968b). To determine if the CFU-S and CFU-C assays detected the same cell type, equilibrium density gradient centrifugation and velocity sedimentation were used to separate mouse BM into various fractions, which were then tested for CFU potential via the in vivo and in vitro CFU assays (Worton 1969). Some cells displayed CFU potential in vivo but not in vitro (and vice versa), suggesting that at least some of the cells responsible for CFU-S are not identical to the cells responsible for CFU-C (Worton 1969). This was one of the first experimental demonstrations of different "classes" of stem and progenitor cells and questioned the validity of the CFU-S method to detect HSCs. However, there was still no conclusive evidence as to what the CFU-C were. CFU-C assays provided the first evidence for a theoretical class of progenitor cells that lay somewhere between an "HSC" (considered the CFU-S at the time of CFU-C discovery) and differentiated granulocytes (Eaves 2015). Later assays that allowed for the detection of other myeloid lineages reinforced this theory (Gregory 1973, Gregory 1976). Assays for the lymphoid potential of progenitor cells were not possible until the work of Dexter in 1977. Here, a co-culture system was developed in which hematopoietic cells were plated with "feeder" cells consisting of BM cells isolated from the femurs of mice (Dexter 1977). Culturing at $33^{\circ} \mathrm{C}$ and periodically replenishing the media and feeder cells allowed for CFU-S and CFU-C potential of the hematopoietic cells to be maintained for several months (Dexter 1977). This was used as a starting point for subsequent studies of ex vivo B-potential and long-term repopulating potential. Indeed, a BM culture method influenced by Dexter was devised in 1981 that allowed for differentiation and maintenance of the B-cell lineage (Whitlock 1982). It was later discovered that clonal lines from this culture method could differentiate adult BM towards the B cell lineage and maintain progenitor cultures for 4 weeks (Whitlock 1987). This provided a way to assay hematopoietic BM for B cell potential ex vivo, as well as a confirmation that this culture method could maintain CFU-S for 3-4 weeks.

In an attempt to identify thymic precursors in the murine BM (established by $\mathrm{Wu}$ 1968a), Lepault utilized fluorescently labelled fluorescin isothiocyanate (FITC+) BM cells and injected them into mice, followed by isolation of various tissues in recipients to see where the FITC+ cells were found (Lepault 1981). Most of the cells homed to the BM in levels proportional to the number of injected cells, and curiously, Thyl-antibody treatment of BM didn't seem to affect homing. This was initially an establishment of a protocol to detect "lymphoid-specific" BM progenitor cells, but was later recognized as a critical paper in identifying bona fide HSCs. It was also found that Thy1+ cells from donor BM could be visualized in recipient thymus using Thy 1 congenic mouse strains.

Many assays were also devised to test for self-renewal properties of HSCs, as well as to assess the absolute number of HSCs in a given tissue. The ex vivo stromal co-culture methods devised by Whitlock (Whitlock 1987) allowed researchers to assay hematopoietic cells for "cobblestone formation" after adding limiting dilutions of BM 
cells to supportive stroma. These "cobblestones" consisted of a stromal cell base with a clonal colony above and below, and had long-term ( $>4$ weeks) myeloerythroid/B cell potential (Whitlock 1987), and limiting dilutions could be used to calculate the number of these multipotent cells in a given cell solution (Ploemacher, 1989). Self-renewal could, in part, be defined by the ability of transplanted cells to repopulate an irradiated host "longterm" in the BM. In 1980, Harrison reported the "competitive repopulation assay" by mixing murine BM from donors with distinguishable hemoglobin and then monitoring the respective cells in the recipient peripheral blood (PB) (Harrison 1980). This allowed for monitoring of the potential stem cell compartment for several years (Shen 1985, Shen 1986). Today, the standard technique for determining the absolute number of HSC in a particular pool of cells in vivo is the competitive repopulating unit (CRU) assay, which relies on injecting a limiting dilution of cells into an irradiated host to calculate the absolute numbers of HSC based on reconstitution kinetics (Miller 2008).

Together, these in vitro and in vivo techniques and observations-which solidified HSC characteristics and defined the CFU-S assay as primarily a marker of progenitor cells - paved the way for isolation of HSCs via modern techniques such as fluorescenceactivated cell sorting (FACS).

\section{HSC isolation and establishment of a hematopoietic hierarchy}

The development of monoclonal antibodies to label specific hematopoietic lineages followed by FACS was instrumental for the modern-day identification of HSCs. Single cells or populations of cells could be sorted and tested for in vitro and in vivo functional potential. For instance, Coffman developed the B-cell lineage antibody, RA3$2 \mathrm{C} 2$, which marked nearly $100 \%$ of $\operatorname{sIg}^{+} \mathrm{B}$ cells and most plasma cells (Coffman 1981a). RA3-2C2 did not label the CFU-S, but it did label an sIg- population in the mouse BM that contained B cell precursors, based on the ability of these cells to give rise to sIg+ cells in vitro (Coffman 1981a). That same year, Coffman would also report development of the B-cell antibody B220 (Coffman 1981b).

In 1986, the B220 antibody was used in conjunction with the Thy 1 antibodywhich was thought of as a potential HSC marker at the time (Lepault 1981, Ezine 1984) - to isolate two early B lymphocyte precursor populations (Müller-Sieburg 1986). B220- Thy $1^{-}$populations contained B cell precursors that only showed short-term potential in Whitlock-White cultures (Whitlock 1987) and produced pre-B and B cells (Müller-Sieburg 1986). Incredibly, the addition of Thy $1^{\text {lo }}$ cells to Whitlock-White cultures resulted in long-term culture and differentiation into several lineages (MüllerSieburg 1986). This led to the hypothesis that the long-term culture initiation Thy $1^{\text {lo }}$ cells, being devoid of B-cell lineage markers, may also be negative for other lineage markers. Indeed, they found that Thy $1^{10}$ Lineagee cells were enriched for long-term culture initiation, CFU-S potential, long-term repopulation potential, and could give rise to all lineages in the spleen, BM, and thymus of irradiated hosts (Müller-Sieburg 1986). These cells also showed self-renewal capacity via serial transplantation (Müller-Sieburg 1986). Therefore, Thy $1^{10}$ Lineage ${ }^{-}$cells were considered one of the first true HSC populations. 
In an attempt to further enrich the HSC, Spangrude et al. obtained several monoclonal antibodies produced against Thy $1^{+}$BM hybridomas (Aihara 1986) and found that an antibody recognizing the surface marker now designated as "stem cell antigen-1" (Sca-1) separated the murine Thy $1{ }^{\text {lo }}$ Lineage- population into two subsets: $\sim 20-30 \%$ Sca$1^{+}$and $\sim 70-80 \%$ Sca- $^{-}$(Spangrude 1988). The Sca-1+ population appeared to contain all of the HSC capacity, and they only needed to inject 40 Thy $1{ }^{\text {lo }}$ Lineage-Sca- $1^{+}$cells to achieve multilineage reconstitution of irradiated murine hosts (Spangrude 1988). It was subsequently shown via single-cell transplants that $1 / 13$ Thy $1{ }^{\text {lo }}$ Lineage Sca- $1^{+}$cells showed $>1 \%$ multilineage reconstitution from 3-7 weeks post-transplantation, and a smaller subset showed long-term reconstitution (>20 weeks) (Smith 1991). This showed that only a subset of the newly defined HSC population were true HSC based on multipotency and self-renewal (Smith 1991). It would also later be confirmed (Uchida 1994) that these cells contributed to both short-term and long-term engraftment in irradiated mice. Shortly thereafter a monoclonal antibody for c-Kit-a receptor tyrosine kinase important for hematopoiesis - was produced (Ikuta 1992). When added to the

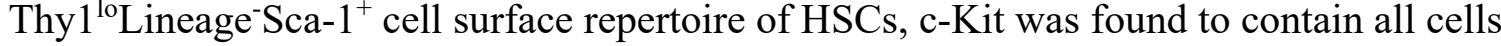
with long-term multilineage reconstitution potential in the murine adult BM and fetal liver (FL) (Ikuta 1992).

In 1994, Morrison first isolated a "subset" of HSCs, termed the "long-term HSC" (LT-HSC) which, by in vivo reconstitution assays, was reported to be c-Kit ${ }^{+} \mathrm{Mac}-1^{-} \mathrm{CD} 4^{-}$ (Morrison 1994). Other subpopulations [c-Kit"Mac- $1^{\text {lo }} \mathrm{CD}^{-}$( (short term-HSCs "STHSCs") and c-Kit"Mac- $1^{10} \mathrm{CD} 4{ }^{\text {lo }}$ (multipotent progenitors "MPPs")] were shown to be enriched for transiently reconstituting cells post-transplantation, and the LT-HSC subset was also shown to be in cell cycle less frequently (Morrison 1994). Through a series of transplantation assays, Morrison was able to show that LT-HSCs give rise to ST-HSCs and MPPs, while MPPs cannot self-renew in the BM of recipient mice (Morrison 1997). This established one of the first hierarchies of hematopoietic stem and progenitor cells (HSPCs), with LT-HSCs established at the top of the hierarchy, followed by ST-HSCs and MPPs. Around the same time, other multipotent and oligopotent progenitors were isolated and assayed for differentiation and self-renewal capacity. In 1997, a common lymphoid progenitor (CLP) that could reconstitute lymphoid but not myeloid cells in vivo at levels $>1 \%$ for $\sim 14$ weeks was isolated based on expression of the Il-7 receptor and reduced Sca-1 and c-Kit expression (Kondo 1997). Shortly thereafter, a hierarchy of myeloid progenitors was identified through $\mathrm{CFU}-\mathrm{C}$ and in vivo repopulating assays (Akashi 2000), in which a common myeloid progenitor (CMP) gives rise to granulocyte/macrophage progenitors (GMP) and megakaryocyte/erythroid progenitors (MEP). These studies provided the basis for the classical model of hematopoiesis, in which HSCs sit atop the hierarchy and give rise to progenitors that become progressively more specified for production of mature hematopoietic lineage cells (Figure 1-2). However, this classical model relied on immunophenotype to identify cell types and was also primarily established on the functional abilities of bulk cell types. Further enrichment of the HSC compartment provided clues that perhaps the contribution of the HSPC compartment to the hematopoietic hierarchy is more complicated than previously appreciated. 


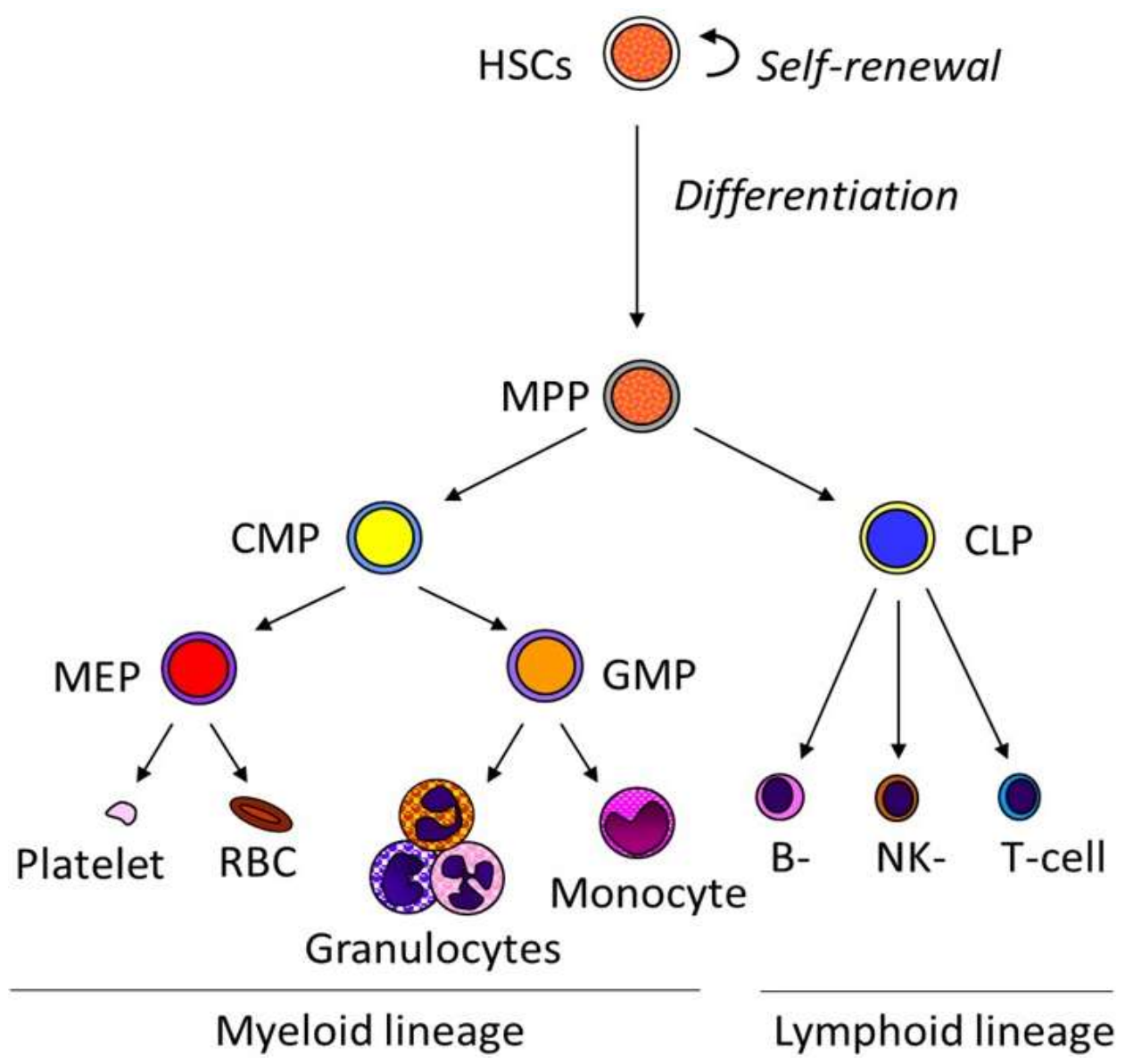

Figure 1-2. The classic model of hematopoiesis

The classic model of hematopoiesis established in the early 21 st century, in which the HSC sit atop the hierarchy and gives rise to progressively differentiated progeny. HSC, Hematopoietic stem cells; MPP, Multipotent progenitor; CMP, Common myeloid progenitor; CLP, Common lymphoid progenitor; MEP, Megakaryocyte/erythroid progenitor; GMP, Granulocyte/macrophage progenitor 


\section{A revised model of hematopoiesis}

Several efforts were made - and are still ongoing - to enrich the LT-HSC compartment for cells with the most potent self-renewal and differentiation properties, primarily through the use of different cell surface markers followed by tests for potency via $\mathrm{BM}$ transplant assay. The phosphoglycoprotein CD34 was recognized as a marker to isolate murine $\mathrm{HSCs}$, as CD34 ${ }^{-}$ineage ${ }^{-} \mathrm{Sca}-1^{+} \mathrm{c}-\mathrm{Kit}^{+}$(CD34 $\left.{ }^{\mathrm{lo} /} \mathrm{LSK}\right) \mathrm{BM}$ cells contained all of the long-term multilineage reconstituting potential in murine $\mathrm{BM}$, and transplantation of single cells showed that the CD $34^{\mathrm{lo} /}$ LSK population had a LT-HSC frequency of $\sim 20 \%$ (Osawa 1996). CD34 had previously been identified as a positive marker on human HSPCs (Baum 1992). Morrison identified a family of cell surface receptors (SLAM) that could be utilized to differentiate between highly purified HSCs and MPPs lacking self-renewal ability (Kiel 2005). All long-term multilineage repopulating cells within the LSK compartment were CD150 ${ }^{+}, \mathrm{CD} 244^{-}$, and CD48- and similarly to the CD34 $4^{\text {lo/- }}$ population (Osawa 1996), $20 \%$ of LSKCD48-CD150 ${ }^{+}$(SLAM) cells had long-term multilineage potential (Kiel 2005). CD48-CD150- cells were considered "MPPs", and the majority of LSKs were found to fall in the CD48 ${ }^{+} \mathrm{CD} 150^{-}$ compartment (Kiel 2005). While experimenting with the vital dye, Hoescht 33342 , Goodell identified a small population of cells that was apparent when visualizing dye fluorescence simultaneously at the red and blue emission wavelengths (Goodell 1996). This "side population" (SP) of cells showed low expression of lineage markers and high expression of Sca-1, and showed a 1000-fold enrichment of long-term multilineage reconstitution compared to whole BM. By subsetting the Hoescht SP based on Rhodamine 123, which had been used previously to prospectively identify HSCs with blue wavelength Hoescht dye, (Wolf 1993), others were able to further enrich the SP HSC population to a level in which $\sim 22 \%$ of mice injected with single SP cells showed long-term multilineage engraftment (Uchida 2003). After performing microarray analysis for differentially expressed genes in murine BM, it was found that endothelial protein $\mathrm{c}$ receptor $(E p c r)$ was upregulated in $\mathrm{HSCs}$, and it was found that $\mathrm{EPCR}^{+}$cells contain functional LT-HSCs (Balazs 2006). Later, it was found through single cell transplants that $\mathrm{EPCR}^{+}$SLAM cells (ESLAM) cells showed long-term multilineage repopulation in $\sim 30 \%$ of irradiated recipients (Kent 2009). These studies provided the basis for how we isolate murine HSCs to this day.

Even within the enriched HSC subpopulations, functional heterogeneity can be observed. By culturing HSCs with a supportive stromal cell line to produce clonallyderived colonies and injecting clonal colonies into mice to visualize their self-renewal and lineage reconstitution capacities, it was found that clonal HSCs have intrinsic properties that are continued through serial transplantation, including reconstitution dynamics and lineage bias (Müller-Sieburg 2002). Indeed, three subtypes of HSCs could be identified: Lymphoid-biased (Ly-bi), Myeloid-biased (My-bi), and Balanced HSCs, based on repopulation kinetics (Müller-Sieburg 2002). These HSCs produce long-term multilineage repopulation but are skewed towards enhanced production of a particular lineage. My-bi HSCs appeared to persist longer in serial transplantation assays in terms of their white blood cell production, produced a reduced number of lymphoid precursors in ex vivo stroma co-culture, and lymphoid progeny from My-bi HSCs (post-transplant) 
displayed reduced levels of IL7 receptor (IL7R) expression (Müller-Sieburg 2004).Using the SP definition, Dykstra isolated HSCs and injected as single cells or as in vitro generated clonal colonies into recipients followed by serial transplantation to identify the longevity and lineage output of clones (Dykstra 2007). Interestingly, he identified several subtypes of HSCs, including myeloid-biased $\alpha \mathrm{HSCs}$, balanced $\beta \mathrm{HSCs}$, lymphoidbiased $\gamma \mathrm{HSCs}$, and short-term repopulating, lymphoid biased $\delta$ HSCs (Dykstra 2007). It was also later found that $\alpha \mathrm{HSCs}$ appear to be underrepresented in fetal tissues at the expense of lymphoid-biased HSCs (Benz 2012). von Willbrand factor $\left(v W F^{+}\right)$HSCs display a significant bias towards platelet/myeloid production after transplantation, and appear to be upstream of $v W F^{-}$HSCs during development, based on the lack of $v W F^{-}$ HSCs in the E14.5 FL but presence of $v W F^{-}$HSCs in the adult BM (Sanjuan-Pla 2013). vWF is a glycoprotein involved in platelet aggregation. Several other studies have identified lineage-biased HSCs in murine BM (Benveniste 2010, Morita 2010, Lu 2011, Oguro 2013).

There also exists evidence for unipotent and/or bi-potent long-term repopulating cells within different immunophenotypically defined HSC compartments. Yamamota et al. established a theory of "myeloid-bypass" in the CD150 ${ }^{+} \mathrm{CD} 31$-LSK murine BM compartment via single-cell transplantation assays (Yamamota 2013). They identified myeloid -restricted progenitors with long-term repopulating activity ( $>16$ weeks, and in some cases, could be secondarily transplanted) in their immunophenotypic HSC compartment by visualizing all lineages with a fluorescent genetic marker (Yamamota 2013). This helped reshape the way people thought about how the hematopoietic hierarchy is established. The SLAMCD $34^{-} \mathrm{CD} 41^{\mathrm{hi}}$ subpopulation of HSCs displayed unipotent, transient reconstitution of irradiated recipients for the megakaryocytic lineage, again suggesting that there is a subset of HSCs that bypass other differentiation programs (Haas 2015). Interestingly, lineage fate did not seem to be affected by inflammatory stress, but this population could be activated to replenish platelets under inflammatory stress (Haas 2015). Using genetically labelled $v W F^{+}$HSCs in single cell transplantation assays followed by monitoring the long-term reconstitution and lineage potential of the cells for all lineages, it was found that $v W F^{+}$HSCs have long-term unipotent platelet and bipotent platelet/erythrocyte potential (Carrelha 2018). The lineage relationships between $v W F^{+}$HSCs and downstream progenitors in recipient bone marrow reflected the reconstitution kinetics seen in the peripheral blood (PB) (Carrelha 2018). These studies suggest that there are subsets of HSCs that maintain self-renewal potential while simultaneously restricting their lineage potential.

The utilization of modern techniques such as single cell RNA-sequencing (scRNA-Seq) to define the transcriptional landscape of the heterogeneous HSPC compartment has provided evidence that lineage commitment may be a continuous process. scRNA-Seq and unbiased clustering of several HSPC populations in the murine BM identified HSCs as clustering in part with MEPs, as well as lymphoid-primed multipotent progenitors (LMPPs) (Moignard 2013). In another study, scRNA-Seq of several murine BM progenitor cells revealed semi-distinct transcriptional signatures between HSCs and downstream progenitors but revealed that there seems to be a gradual regulation of gene expression across HSPC commitment (Nestowara 2016). 
This supports a model in which the HSPC hierarchy resembles more of a "continuous" commitment process verses a "stepwise" commitment process, and this continuous process was also observed in human BM CD $34^{+}$cells via combined scRNA-Seq and single cell ATAC-Seq data (Buenrostro 2018)

Compositional and functional heterogeneity has also been observed in the progenitor compartments downstream of HSCs. After previously showing that the receptor tyrosine kinase Flt3 labels one-half of the LSK compartment and that Flt3 ${ }^{+}$LSK primarily display lymphoid commitment (Adolfsson 2001), Adolfsson et al. enriched for LSKFlt $3^{+}$cells and showed that they possessed lymphoid and myeloid lineage production capacity in vitro and in vivo (Adolfsson 2005). However, LSKFlt3 ${ }^{+}$cells do not have the capacity to produce megakaryocyte/erythroid lineages. They termed this LSKFlt ${ }^{+}$ population the "LMPP," and suggested that it is downstream from ST-HSCs, gives rise to all lymphoid cells, and either replaces CMPs or has the potential to give rise to GMPs (Adolfsson 2005). This was one of the first challenges to the bi-directional model of lineage production in the hematopoietic hierarchy. However, the concept of an LMPP was challenged a year later, when Forsberg and colleague showed that transplanted LMPP do give rise to platelets and erythroid cells using a fluorescent genetic marker (Forsberg 2006). This same group would later claim that all hematopoietic cells during steady state arise from a Flt3 ${ }^{+}$precursor, suggesting Flt3 expression is integral to blood production (Boyer 2011).

Some groups have even further subdivided the MPP compartment downstream of the HSC. The lab of Andreas Trumpp identified 4 subpopulations of MPPs based on cell surface expression and varying functional assays: MPP1 (LSKCD34 $4^{+} \mathrm{CD} 48^{-} \mathrm{CD} 150^{+} \mathrm{Flt3}^{-}$ ), MPP2 (LSKCD34 ${ }^{+} \mathrm{CD} 48^{+} \mathrm{CD} 150^{+} \mathrm{Flt3}^{-}$), MPP3 (LSKCD34 ${ }^{+} \mathrm{CD} 48^{+} \mathrm{CD} 150^{-} \mathrm{Flt3}^{-}$), and MPP4 (LSKCD34 ${ }^{+} \mathrm{CD} 48^{-} \mathrm{CD} 150^{+} \mathrm{Flt}^{+}$) (Wilson 2008). Via label-retaining studies during homeostasis, they found that the HSC (LSKCD34-CD48 ${ }^{-} \mathrm{CD} 150^{+} \mathrm{Flt3} 3^{-}$) at the top of the hierarchy was dormant, and they hypothesized that upon activation, CD34 expression increases and differentiation down the pathway (MPP1-MPP4) begins (Wilson 2008). The Morrison lab utilized CD229 and CD224 SLAM markers to subset the ST-HSC compartment and found that $\mathrm{CD} 229^{-} \mathrm{CD} 224^{-}$(MPP1) cells appeared to sit at the top of the MPP hierarchy, as upon transplantation they were able to give rise to MPP1, $\mathrm{CD}_{22} 9^{+} \mathrm{CD} 244^{-}$(MPP2), and CD229 ${ }^{+} \mathrm{CD} 244^{+}$(MPP3) (Oguro 2013). Meanwhile, MPP2 and MPP3 cells could not give rise to other MPP populations, but were able to transiently reconstitute irradiated recipients and self-renew in the BM (Oguro 2013). In perhaps the most accepted study of different MPP populations, Pietras et al. sorted 3 MPP populations and tested in vitro and in vivo lineage potential, as well as lineage hierarchy among the MPP populations and the dynamics of MPP production by LT-HSCs (Pietras 2015). They found that LSKCD $48^{+} \mathrm{CD} 150^{+} \mathrm{Flt3} 3^{-}$(MPP2) cells displayed a myeloid biasparticularly towards the megakaryocyte/erythroid lineages - in CFU assays and after

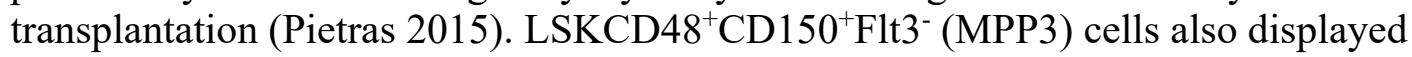
myeloid bias but had lower meg/erythroid lineage potential, while MPP4 cells $\left(\mathrm{LSKCD} 48^{+} \mathrm{CD} 150^{-} \mathrm{Flt3}^{+}\right.$) were lymphoid-primed with minor myeloid potential (Pietras 2015). Importantly, they showed that HSCs could produce all MPP subtypes, but MPPs could not produce other MPPs in transplantation assays (Pietras 2015). Also, in in vitro 
differentiation assays and presumably after transplantation, HSCs establish the myeloid biased MPPs (MPP2/3) first, followed by establishment of MPP4s to reconstitute the lymphoid lineage (Pietras 2015). The nomenclature used to describe the MPP subtypes put forth by Pietras et al. is currently the most widely accepted.

Heterogeneity in the HSC and MPP compartments in the BM have led to a revised model of the hematopoietic hierarchy, in which multiple HSPC subtypes contribute to a variety of lineages, and even some HSCs can bypass differentiation into progenitor subtypes to produce mature cells of the megakaryocytic lineage (Figure 1-3).

\section{Summary}

The discovery of HSCs took decades to uncover, and the process for their discovery is the model by which many other stem cell biology fields follow to identify stem cells. HSCs have come to be defined as a cell that has self-renewal and multilineage potential, and the gold standard for assaying these properties has become HSCT. Therefore, the standard HSC definition has also come to include the ability to home and repopulate the BM of an irradiated recipient, although recent studies suggest that HSC function during steady state and transplantation may widely vary. Immunophenotypic HSCs are clearly a heterogeneous group of cells that may not all conform to the properties derived from HSCT, and ongoing work even suggests that the transplantable HSC may be a unique HSC subtype itself. Nevertheless, the utility of HSCT in the clinic has drastically improved the lives of patients with hematological maladies. Finally, the composition and trajectory of the hematopoietic hierarchy-especially at the level of the HSPC compartment - is constantly being challenged by the identification of new cellular subtypes and the boom of single cell analysis techniques. More research is needed to elucidate the nuances of the hierarchical structure of hematopoietic cells.

\section{Where Do Hematopoietic Cells Come From?}

The observation of blood within the yolk sac (YS) of many species during early embryonic development (termed "blood islands") in the late $19^{\text {th }}$ century suggested that the hematopoietic system had an extraembryonic origin (Medvinsky 2011). It wasn't until 1975 that this was seriously challenged, when Francoise Dieterlen-Lievre grafted whole quail embryos to chick blastoderms and showed that quail hematopoietic organs were not colonized by hematopoietic cells from the chick yolk-sac (Dieterlen-Lievre 1975). He also showed that, when grafting quail spleen or thymus into the chick embryo coelom, the hematopoietic organs of the quail were populated by chick blood cells (Dieterlen-Levre 1975). Dieterlen-Lievre performed these grafts at pre- and post-circulation timepoints and found similar results and suggested that the YS blood islands only contain primitive erythrocytes while the embryo proper contain "definitive" stem cells (Dieterlen-Lievre 1975). Since Dieterlen-Lievre's work, hematopoietic establishment in the embryo has been thought to occur in successive waves: two "primitive" waves and a "definitive" wave. An establishment of the properties of a bona fide HSC (reviewed above) has 


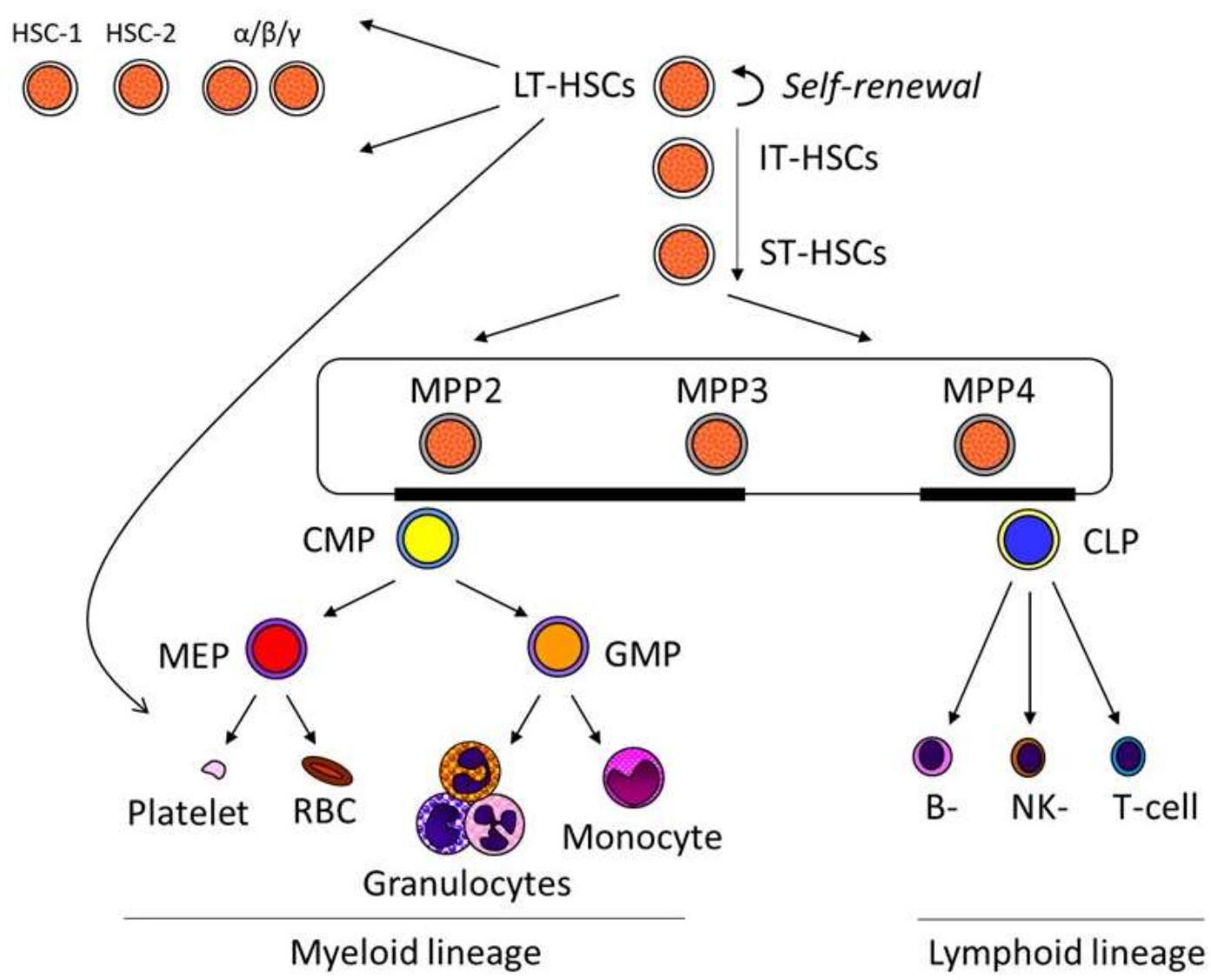

Figure 1-3. The revised hematopoietic hierarchy

The revised model of hematopoiesis established by enrichment and functional assessment of different HSC and HSPC compartments. This model suggests the presence of lineagebiased and lineage-bypassing HSCs, as well as a more fluid production of the different committed downstream progenitors. LT, Long-term; ST, Short-term; IT, Intermediateterm; HSC, Hematopoietic stem cells; MPP, Multipotent progenitor; CMP, Common myeloid progenitor; CLP, Common lymphoid progenitor; MEP, Megakaryocyte/erythroid progenitor; GMP, Granulocyte/macrophage progenitor. 
allowed researchers to assay embryonic tissues for the origins and presence of the "definitive HSC" (dHSC), marking the beginning of definitive, lifelong hematopoiesis. In short, a dHSC is defined as a cell that has long-term multilineage reconstituted potential in an irradiated recipient. Here, I will review the primitive and definitive waves of hematopoiesis, with some speculation on whether the dHSC represents the true cell that sustains lifelong hematopoiesis throughout the life of the organism.

\section{The first primitive wave is established by the hemangioblast}

In 1920, Sabin noticed that YS blood islands could first be seen in mice at embryonic day 7.5 (E7.5) and appeared to be clusters of erythrocyte progenitors surrounded by endothelial progenitors (Sabin 1920). This generated a hypothesis of the "hemangioblast," which is a bipotent progenitor of endothelial and blood cells. It is worth noting that the term "blood island" in murine embryos has since been deemed a misnomer by many in the field (Medvinsky 2011). Discrete islands were enticing to researchers searching for the presence of hemangioblast "clones," but the appearance of multiple islands was due in part to the sectioning method of mouse embryos, as whole mount sections showed that the blood "islands" were actually a single blood "band" encircling the E7.5 embryo (Ferkowicz 2005). However, this did not negate the possibility of the islands being formed from a bipotent progenitor.

In 1997, an avian monoclonal antibody targeting vascular endothelial growth factor receptor-2 (VEGFR2) - a tyrosine kinase receptor required for endothelial and hematopoietic production in mice-was developed to isolate VEGFR2 ${ }^{+}$mesodermal cells that eventually colonize the YS from gastrulating chick embryos (Eichmann 1997). These mesodermal cells could produce either hematopoietic or endothelial colonies in vitro, but not both (Eichmann 1997). A similar conclusion - that hematopoietic and endothelial cells had different developmental origins in the YS - was also proposed in the mouse (Kinder 1999). The first experimental evidence of the hemangioblast came from an in vitro embryonic stem cell culture system, in which individual blast cell colonies from embryoid bodies were exposed to VEGF and produced endothelial and hematopoietic precursors (Choi 1998). These "blast colony adherent cells," expressed endothelial markers, including CD31, and were considered the hemangioblast (Choi 1998). The existence of a hemangioblast was later also demonstrated using human ES cell cultures (Kennedy 2007).

Drake et al. demonstrated that hematopoietic and endothelial cells of the E7-E7.5 mouse YS blood island show co-expression of some genes, particularly the transcription factor Tall, which is also expressed by embryonic-derived "angioblasts," thought to be the precursors to endothelial cells (Drake 2000). In 2004, Huber performed pivotal experiments in which portions of the YS, posterior/distal primitive streak, anterior region, and lateral domains of E7-7.5 mouse embryos were dissected to try to determine where the hemangioblast was located in vivo (Huber 2004). They cultured these tissues in a similar manner as Choi to see which tissue contained hemangioblasts. Incredibly, they noted that the greatest hemangioblast potential came from $\mathrm{Flkl}^{+}(\mathrm{Vegf}) \mathrm{Brachyury}^{+}$cells in 
the primary primitive streak (Huber 2004). They also concluded that these cells arise from mesodermal (Brachyury ${ }^{+}$) cells in the distal primitive streak and have endothelial, hematopoietic, and vascular smooth muscle potential (Huber 2004). This suggested that by the time the hemangioblasts have entered the YS from the embryo proper they have essentially all segregated into either hematopoietic or endothelial potential, showing that the hemangioblast is specified independently of the YS. Subsequent studies used genetic lineage tracing to suggest that not all endothelial cells in YS blood islands come from hemangioblasts, but rather a committed endothelial progenitor (Ueno 2006). However, this was not performed in embryos de novo, but rather using ES cells placed in normal blastocysts to form tetrachimeras (Ueno 2006). Together, these studies confirmed the presence of a hemangioblast that could give rise to endothelial and hematopoietic cells, and the current model for the generation of YS blood islands includes seeding of the YS by embryo-derived hemangioblasts (Figure 1-4).

\section{The first primitive wave: Blood cell types and their fate}

Primitive red blood cells (RBCs) are perhaps the best studied cell type of primitive hematopoiesis. Primitive erythrocytes differ from their adult counterparts (which do arise in the fetus) via the hemoglobin chains they express. Primitive RBCs express $\beta \mathrm{H} 1$ and $\varepsilon$ chains, whereas adult RBCs mainly express $\beta$ and $\alpha$ hemoglobins (Craig 1964, Wong 1983). The primitive erythrocytes are nucleated and dominate the circulating blood at E12, whereas adult erythrocytes are enucleated and are the predominant RBC by E15. (Craig 1964, Wong 1983). In an elegant demonstration, McGrath et al. stained for erythroblasts in the YS and embryo proper at early time points and found that erythroblast presence occurs right around the time the embryonic heart starts beating, $\sim \mathrm{E} 8.25$ (McGrath 2003). In 2004 it was found that primitive $\beta \mathrm{H} 1^{+}$ erythrocytes can be found circulating in the postnatal day 5 (P5) blood of mice and enucleate around E12.5-E16.5, so they may not necessarily require an erythroblastic island and central macrophage for enucleation (Kingsley 2004). It was later found that primitive erythrocytes home to and preferentially reside in the FL around the time of enucleation, and it is thought that they interact with erythroblastic islands surrounding FL macrophages to induce enucleation (Isern 2008, McGrath 2008). Thus, primitive RBCs are thought to originate from the YS blood islands, enter the embryo proper around the time of circulation, continue to be present in neonatal tissues, and finish their maturation process in the FL niche.

Megakaryocytes are also thought to arise during the first primitive wave of hematopoiesis. Primitive, low ploidy megakaryocytic progenitors can be detected in mouse YS from E7.5-E10.5, with little to no primitive megakaryocytic progenitors in the embryo body or FL (Xu 2001). Meanwhile, megakaryocyte progenitors thought to arise from the definitive hematopoietic wave have been reported in the YS at E10.5 and may be the dominant progenitor type in the E13.5 FL (Xu 2001). Tober et al. found that E7.5 hemangioblasts contained megakaryocytic potential, and they used CFU assays coupled with immunohistochemical analysis for megakaryocytes and primitive/definitive erythrocytes to show that at E7.25, a primitive MEP arises in the YS, followed by what 


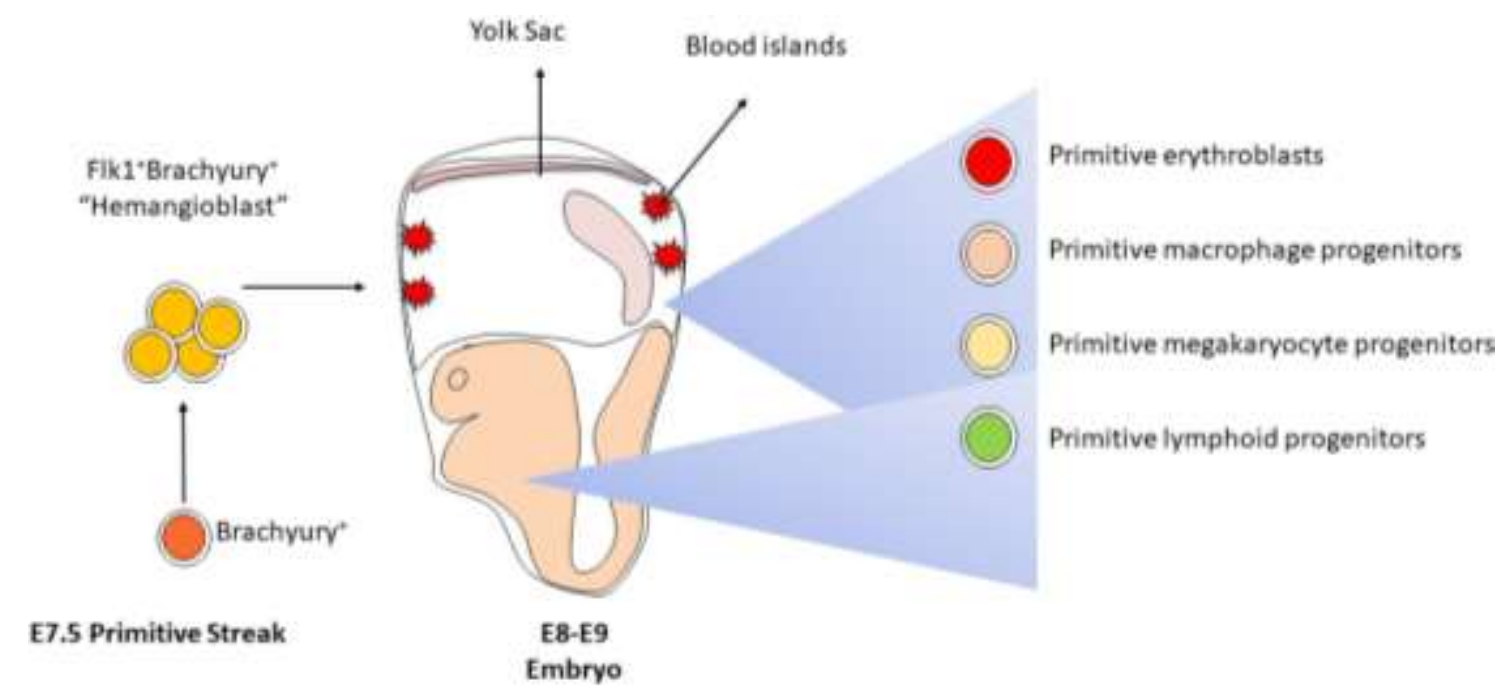

Figure 1-4. The first primitive wave of hematopoiesis

The first primitive wave of hematopoiesis is thought to be mostly confined to hemangioblast-derived yolk sac blood islands. These yolk sac blood islands produce primitive erythroblasts, macrophage progenitors, megakaryocyte progenitors, and putative lymphoid progentiors around E8-E9 in the mouse. Interestingly, the lymphoid progenitors may also arise from the embryo proper at $\sim \mathrm{E} 9$. 
they claimed to be a definitive MEP (also in the YS) that contributes to definitive megakaryopoiesis and erythropoiesis (Tober 2007). Platelets were found to be circulating in the embryonic vasculature at E10.5, and the site of megakaryocyte progenitors shifts from the YS to the FL at E10.5 (Tober 2007). Primitive megakaryocytic progenitors would later be termed "diploid platelet-forming cells" (DPFCs) due to their reduced ploidy (Potts 2014). It was later shown that DPFCs actually appeared in the YS at E8.5, followed by migration to the embryo body, placenta, and PB by E9.5 (Potts 2015). Highly polyploid megakaryocytes did not emerge until E10.5 in the FL, so all platelet production at earlier time points was due to primitive megakaryocyte progenitors (Potts 2015). In the most interesting revelation from this study, DPFCs and definitive megakaryocytic progenitors displayed similar transcriptional programs, but DPFCs did not require thrombopoietin (TPO) for platelet production while definitive megs did (Potts 2015). In an even more recent study, embryonic megakaryocytes appeared to be CD45while adult $\mathrm{BM}$ megakaryocytes are $\mathrm{CD} 45^{+}$(Cortegano 2019). Together these studies suggest that megakaryocytes from the first primitive wave are TPO-independent, lowploidy, CD45- cells that can still produce a wave of platelets by E8.5, and definitive megakaryopoiesis occurs in the FL after either a maturation of the primitive lineage or production of a new wave of megakaryocytic progenitors from a different embryonic tissue.

Two papers from Naito and Takahashi identified the first macrophages in the mouse embryo. In 1989, Takahashi showed that primitive macrophages first arise in the YS blood islands at E9, and appear to differentiate immediately into what were termed "fetal macrophages," at E10 (Takahashi 1989). The fetal macrophages don't appear to have phagocytic properties and are specified prior to primitive monocytes (which arise after E10), suggesting that they bypass the typical myelopoietic pathway of macrophage production seen in the adult BM (Takahashi 1989). The next year, Naito found fetal macrophages - presumably derived from migratory primitive macrophages from the YS - in the E10 FL (Naito 1990). However, they also found a "definitive" macrophage population that appeared to be derived from monocytes, similar to the differentiation pathway seen in the adult BM (Naito 1990). Therefore, they hypothesized that there were two "waves" of macrophage production, which definitively have YS origins but may also have FL origins (Naito 1990). Later, Bertrand et al. showed that the "primitive" macrophages described by Naito and Takahashi were actually maternally derived via a biallelic mating scheme (Bertrand 2005a). They also proved via genetic fluorescent labelling of macrophages in vivo that a population of $\mathrm{c}-\mathrm{Kit}^{+}$macrophage progenitors in the E7.5-8 YS are the first true "primitive" macrophage precursors, as they gave rise to unipotent macrophage colonies in methycellulose assays (Bertrand 2005a). These cells were found to be in the YS as late as E10 and were shown to mature through a monocytic pathway like the "definitive" macrophages of Naito and Takahashi (Bertrand 2005a). Finally, macrophages derived from the YS-derived primitive macrophage precursors at E8 appeared to enter circulation and invade the embryo proper by E9, as determined by a macrophage-specific fluorescent marker in transgenic mice (Ovchinnikov 2008). In sum, the YS is the initial site of the first primitive wave of macrophages at E8, which appear to follow a differentiation pathway that is similar to adult macrophages. 
There is also some evidence that a population of lymphoid progenitors arises during the first primitive wave. E9 YS and para-aortic splanchnoleura (P-Sp, a tissue that will eventually give rise to the dorsal aorta and urogenital ridges) from $\mathrm{Ncxl}^{-/-}$mice, which lack a heartbeat, were transplanted into the peritoneal cavity of neonatal mice to assay for the presence of B-cell progenitors (Yoshimoto 2011). Interestingly, both tissues could produce functional B1 progenitors that primarily gave rise to B1a cells, and that cells positive for the endothelial marker VE-Cadherin from these same tissues could produce B1 progenitors in vitro (Yoshimoto 2011). The same lab would perform similar experiments in 2012 and discover transient reconstitution of host (neonatal) thymus, and that these progenitors could arise from embryonic VECadherin ${ }^{+}$cells (Yoshimoto 2012). Later, dHSC-deficient mice (McGrath 2015) were used to confirm these findings, as well as showing that these dHSC-independent B1 progenitors populate the FL (Kobayashi 2014a).

The first primitive erythroid, megakaryocytic, and macrophage lineages are YSspecific, and are thought to derive from a tri-potent "hemangioblast" that originates in the primitive streak of the embryo proper followed by migration to the YS (Figure 1-4). These cells appear to have different properties from their adult counterparts, which may be due to their relatively rapid expansion in the pre-circulation embryo in preparation for oxygen transport or tissue maintenance. Meanwhile, the first primitive lymphoid progenitors appear to have the ability to arise from endothelial cells of the YS and P-Sp prior to dHSC formation (Figure 1-4).

\section{The second primitive wave: Multipotent progenitors that are distinct from dHSCs}

The second primitive wave of hematopoiesis is mainly characterized by the production of erythromyeloid progenitors (EMPs) in the YS. In 2001, James Palis identified high-proliferative potential colony forming cells (HPP-CFC) in the E8.25 precirculation YS, which produce large, multilineage colonies in methylcellulose culture (Palis 2001). This confirmed that there is a first primitive wave of hematopoiesis, as several unipotent progenitors can be found in the YS prior to E8.25 (Palis 2001). These HPP-CFC appeared in the bloodstream of the embryo proper after circulation but were still predominantly found in the YS until the FL became the main site of HPP-CFC production at E11.5 (Palis 2001). When cultured in methylcellulose, HPP-CFC produced erythroid/macrophage colonies, suggesting they had erythromyeloid potential (Palis 2011). These were considered the first multipotent progenitors found in the developing mouse embryo and would later be called EMPs.

After the discovery of the EMP, attempts were made to draw similarities with the population and the $\mathrm{dHSC}$ (reviewed below). It was previously found that deletion of the transcription factor Runxl (Okuda 1996) and its binding factor Cbf $\beta$ in mice resulted in the depletion of cells that would be later considered EMPs (Okuda 1996) as well as dHSCs, with only primitive erythropoiesis remaining. In 2004, Hadland et al. found that mice lacking the Notch1 receptor, which is involved in cell fate decisions throughout development, produced EMPs but did not produce dHSCs via CFU-assay (Hadland 
2004). This was thought of as a potential developmental checkpoint in the pathway to achieving a dHSC in vivo but could certainly have also meant that EMPs and dHSCs required different transcriptional mechanisms for their generation. EMPs would later be isolated from E10.5 YS as CD45 ${ }^{\text {lo }} \mathrm{CKit}^{+} \mathrm{AA} 4.1^{+}$cells, as this population contained nearly all of the EMP potential via CFU assays (Bertrand 2005b). This was also considered the immunophenotypic dHSC population at the time, since these cells could also form lymphoid colonies in vitro (Bertrand 2005b). It would later be found that EMPs could be enriched via cell-surface expression of CD16/32 (McGrath 2015), which is not expressed on dHSCs (Bertrand 2005b). To determine if the cellular origins of EMPs and dHSCs were similar, Chen et al. 2011 constructed a Runx $1^{-/-}$mouse that could also express a Runx 1-GFP fusion protein from either $\mathrm{Tie}^{+}$(a marker for venous endothelium) or $\mathrm{Sca}-\mathrm{I}^{+}$ (marks arterial endothelium) endothelium to see the effect of different hemogenic endothelium sources on EMP and dHSC formation (Chen 2011). They found that EMPs were rescued by Runx 1 expression in Tie $2^{+}$endothelium while dHSCs were rescued by Runxl expression in $\mathrm{Sca}-\mathrm{I}^{+}$endothelium, suggesting that EMPs and dHSCs arise from different hemogenic endothelial sources (Chen 2011). This was further confirmed via the differential expression of hepatic leukemia factor $(H l f)$, which is required for dHSC specification but not for EMP specification (Yokomizo 2019). Importantly, it was also shown that dHSCs and YS are functionally distinct in vivo via transplantation of explant cultures of YS and P-Sp (which is the precursor to the dHSC niche, reviewed below) from pre-circulation embryos (Cumano 2001). YS showed only transient myeloid reconstitution, while P-Sp showed long-term multilineage reconstitution of recipients (Cumano 2001).

It was also determined that upon generation in the YS, EMP migrate to the FL and constitute a heavy presence by E10.5 (McGrath 2015). It is also suggested that EMPs begin differentiating in the FL, as granulopoietic cells were not seen circulating in the embryo until E11.5, although this data is correlative (McGrath 2015).Therefore, the EMP is the first known multipotent progenitor that arises in the murine embryo, and early attempts to compare it to dHSCs showed that while it does share some properties of developmental regulation (e.g. Runxl-expression) and even likely shares niche space with dHSC in the FL, it is immunophenotypically and functionally distinct, and it almost certainly arises from a different tissue source than the dHSC during development (Figure 1-5).

There is also speculation that lymphomyeloid precursors may exist in the YS as early as E9.5. Boiers et al. immunophenotypically identified a cell with B, T, and Myeloid (but no megakaryocyte/erythroid) potential via liquid culture in the E11.5 FL that was $\mathrm{Lin}^{-} \mathrm{Kit}^{+} \mathrm{Il}^{+} \mathrm{R}^{+} \mathrm{Flt3}^{+}$(Boiers 2013). They used a fluorescent marker to label the cells in vivo and found them in the E9.5 YS but not in other tissues, and these YS lymphomyeloid progenitors expressed transcripts associated with lymphoid and GM priming, but not megakaryocyte/erythroid priming (Boiers 2013). To prove that these cells originate in the YS, they performed explant culture of pre-circulation YS and P-Sp from transgenic mice and saw that only the YS produced lymphomyeloid progenitors in the explant culture (Boiers 2013). Further research needs to be conducted to verify the presence of this YS-derived lymphomyeloid progenitor. 


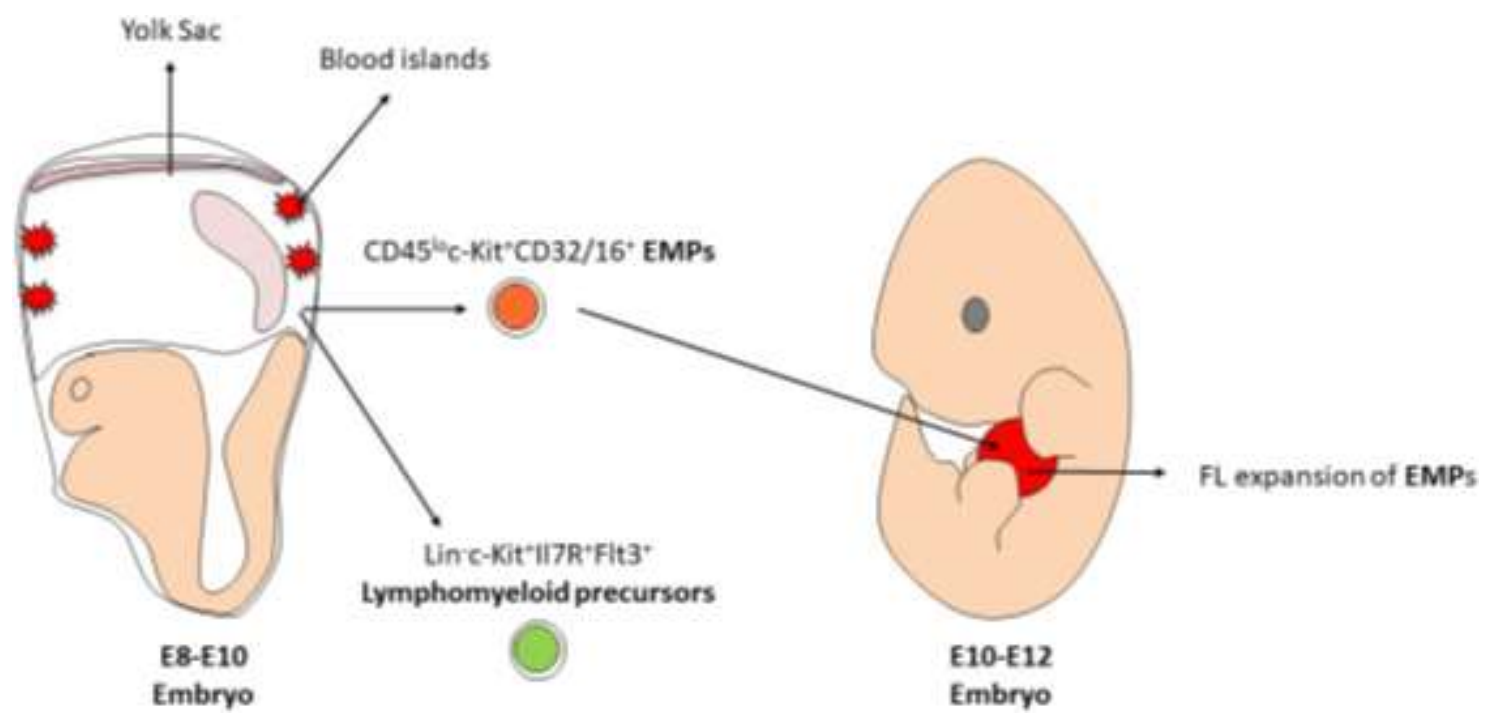

Figure 1-5. The second primitive wave of hematopoiesis

The second primitive wave of hematopoiesis is confined to the yolk sac (YS), and primarily consists of the production of YS-derived erythromyeloid progenitors (EMPs) which migrate to and expand in the $\sim \mathrm{E} 10.5$ fetal liver (FL). There is also evidence of lymphomyeloid precursors (lacking megakaryocytic/erythrocytic potential) arising from the YS around the same time as EMPs. 


\section{The development of the AGM}

The onset of definitive hematopoiesis is thought to occur primarily in the aorta gonad mesonephros (AGM), a region consisting of the dorsal aorta (DA) - the accepted site of definitive hematopoiesis generation - and the urogenital ridges (UGR), of the developing embryo. Using non-mammalian embryos such as zebrafish or Xenopus, several groups were able to easily characterize AGM formation during embryogenesis.

After noticing that Flk-1 knockout (KO) in mice prevented vessel and blood formation (Shalaby 1995), Fouqet et al. cloned the zebrafish Flk-1 homolog to perform in situ RNA hybridization in zebrafish embryos to determine the dynamics of $\mathrm{Flk} \mathrm{I}^{+}$cell formation and fate (Fouquet 1997). They found that soon after gastrulation in the zebrafish embryo, $F l k-1^{+}$cells arise in the posterior lateral mesoderm (PLM) and begin to migrate medially within the embryo (Fouquet 1997). These $F l k-I^{+}$cells will go on to form the DA below the notochord of the zebrafish embryo, as well as other vessels (Fouquet 1997). It was later found that these PLM Flk- $1^{+}$cells also expressed the hematopoietic TFs $S c l$ and Gata2, as well Pax2, a pronephric duct marker (Gering 1998). They also express $\mathrm{Lmo}$, which interacts with $\mathrm{Scl}$ and is critical to hematopoietic development (Dooley 2005). Therefore, these PLM cells that will eventually give rise to the DA appeared to be specified towards an endothelial and hematopoietic fate prior to DA formation. $\mathrm{Flk1}^{+}$cells were also found in Xenopus embryos in a similar area as in zebrafish embryos prior to the formation of the DA (Cleaver 1998). Specifically, in situ hybridization showed that these cells were found in dorsolateral strips of mesoderm (termed the dorsolateral plate (DLP)) and appeared to migrate medially during development to form the DA (Cleaver 1998). Interestingly, Cleaver et al. also found that Vegf (the ligand for Flk1) was a chemoattractant for DLP cells towards the midline of Xenopus embryos (Cleaver 1998). This was later confirmed (Ciau-Uitz 2010), as Vegf is regulated by the Ets transcription factor Etv6. In the absence of Vegf or Etv6, Flk1 ${ }^{+}$DLP cells lack Scl and Gata2 expression, and do not migrate medially to form the DA in Xenopus embryos (Ciau-Uitz 2010).

Later, it was found that loss of Notch-signaling in zebrafish embryos via Notchsignaling mutants or injection of Notch inhibitors does not prevent the formation of the DA in the AGM, but does seem to shift its transcriptional mechanisms, showing an increase in venous with a decrease in arterial gene expression (Lawson 2001). This suggested that Notch signaling is important for proper formation of the DA (Lawson 2001). This was later confirmed, as Burns et al. utilized transgenic zebrafish that displayed no definitive hematopoiesis due to lack of expression of the transcription factors Runxl and $c-M y b$ in the DA to show that Notch is required for the formation of definitive hematopoiesis through rescue expreiments in which Notch was ectopically expressed (Burns 2005).

By utilizing several Hedgehog $(H h)$ signaling pathway mutants and $H h$ inhibitors in zebrafish embryos, Gering et al. found that $H h$ is required for both the migration of $F l k 1^{+}$cells to form the DA, as well as the prospective induction of endothelial programs once the DA has been formed (Gering 2005). Again using zebrafish embryos, Rowlinson 
and colleagues found that the transcription factor Hey2 is expressed in the PLM prior to DA generation, but requires $\mathrm{Scl}$ expression as well as $\mathrm{Hh}$ and $\mathrm{Veg} f$ signaling for its expression (Rowlinson 2010). By using several Hey 2 morpholinos to disrupt Hey2 mRNA, they found that Hey2 is not required for DA formation, but is required for Notch expression in the DA (Rowlinson 2010). Therefore, Hey 2 acts downstream of Flk $1^{+}$PLM specification and migration, but upstream of the Notch programs necessary for definitive hematopoiesis from the DA.

In summary, zebrafish and Xenopus embryos were critical in deciphering the mechanisms behind the formation of the DA in the AGM region, which appears to contain the programs necessary for definitive hematopoiesis. DA forms via the $H h$ and $V e g f$-mediated medial migration of $\mathrm{Flkl}^{+}$lateral mesoderm that expresses endothelial and hematopoietic factors. Notch signaling then induces the necessary endothelial programs required for proper DA contribution to definitive hematopoiesis.

\section{The AGM is the sole source of dHSC formation}

In 1994, Müller et al. performed a series of experiments that were critical for defining the AGM as the sole site of dHSC formation. They isolated several tissues from E10 and E11 mouse embryos to find that the AGM contained long-term multilineage repopulating cells via primary and secondary transplantation assays (Müller 1994). They injected one embryo equivalent (e.e.) AGM into each recipient and found that 3/96 recipients of E10 AGM and 11/19 recipients of E11 AGM showed long-term multilineage engraftment (Müller 1994). Interestingly, no other tissues transplanted (YS, FL, or PB) showed long-term multilineage engraftment at E10, and while their definition of multilineage engraftment was not as stringent as later papers, this was the first evidence that the dHSC was generated in the AGM around E10 (Müller 1994).

A few years later, Medvinsky developed a critical technique for ex vivo culture of embryonic tissues, in which whole tissues are cultured on 0.65 um filters for 2-3 days in myeloid long-term culture media, dissociated with collagenase after culture, then injected into irradiated recipients (Medvinsky 1996a). The rationale behind the assay was that explant cultures would allow for an expansion of any dHSCs that were present in fresh tissues. Using this explant culture and transplantation assays, Medvinsky found that the AGM was the sole site of dHSC formation at this time point. Later, the explant culture system was used to show that peak expansion of dHSC in the AGM seems to occur at E12, and then dissipates by E13 (Kumaravelu 2002). In contrast, expansion of dHSC in the FL seemed to increase at E13 (Kumaravelu 2002). Importantly, it was also determined that the number of dHSC in the total embryonic body at E10 and E11 via limiting dilution assays was $<3$, suggesting a very small number of dHSCs arising from the AGM at the initial dHSC generation timepoints (Kumaravelu 2002). This helped establish the concept of the dHSC arising in the AGM followed by migration and expansion in the FL. Of note, future studies also isolated placenta from early time points in the mouse but could not find dHSC potential until E11 (Ottersbach 2005). 
Sub-dissection of the AGM region followed by transplantation of either fresh or explant culture tissues determined that all of the dHSC activity at E11 was contained in the DA and umbilical/vitelline arteries, while the UGR contained no dHSC until E12 (de Bruijn 2000). This suggested that the arterial regions of the AGM were the sites of dHSC generation, and further confirmed speculation that $\mathrm{dHSC}$ had endothelial origins. Recently, our lab found that dHSC activity could be produced from long-term explant cultures of the pre-circulation embryo body but was not found in pre-circulation YS (Ganuza 2018). This potential in the pre-circulation body presumably comes from the P$\mathrm{Sp}$, as previous studies showed that short-term explant cultures of this region in precirculation embryos gave rise to multipotent but low-repopulating cells (Cumano 2001).

In sum, the AGM is thought to give rise to the first dHSCs, as no other embryonic tissues show dHSC potential as early as $\sim \mathrm{E} 10$. Also, this potential may already present in the pre-circulating embryo, and dHSCs appear to first arise exclusively from an intraembryonic origin.

\section{How does the AGM generate dHSCs?}

Via immunostaining, it was determined that the ventral wall of the DA contains cell clusters in mice (Medvinsky 1996b) and humans (Tavian 1996), and it was determined in chick embryos that these cells were $\mathrm{CD} 45^{+}$, suggesting that they were hematopoietic clusters (Jaffredo 1998). Crucially, Jaffredo et al. proposed that the clusters were of endothelial origin via lineage tracing of embryonic endothelial cells, showing that the clusters were derived from the endothelium (Jaffredo 1998).

Interestingly, the floor of the DA contained similar cells, and some cells were even found in the underlying mesenchyme of the DA (Jaffredo 1998). This suggested that dHSCs in the DA arise from an endothelial progenitor with hematopoietic potential, termed the "hemogenic endothelium."

The ventral wall of the DA in Xenopus and zebrafish display expression of several markers of hematopoietic specification, including Scl, Gata-2, cMyb, and Ikaros, but two critical regulators of cluster and dHSC formation in the AGM are Runxl and Notch1. In 1999, North et al. used a Runxl-reporter mouse to identify Runx $1^{+}$cells in the developing mouse embryo (North 1999). They showed that Runx $I^{+}$cells are present in the DA clusters, some endothelial cells in the ventral wall of the DA, and in the underlying mesenchyme. Most strikingly, Runx $1^{-/}$mice did not form clusters in the DA at E10.5, suggesting that Runxl is required for the generation of hematopoietic clusters from hemogenic endothelium (North 1999). Later studies confirmed the need for Runxl in zebrafish aortic cluster formation (Kissa 2010), as well as hematopoietic generation from ES-cell derived hemogenic endothelium (Lancrin 2009). It was later found that $\mathrm{Notch1}^{-/}$ embryos contain no in vivo repopulating activity from E9.5 P-Sp, as well as impaired formation of vasculature and hematopoietic cells in organ cultures (Kumano 2003). This suggested that Notch1 was important for the generation of AGM-derived hematopoietic cells (Kumano 2003). Indeed, chimeric mice created with $N o t c h 1^{-/-}$cells at the blastocyst stage showed that Notch1 is dispensable for primitive hematopoiesis, but KO cells failed 
to contribute to definitive hematopoiesis in the FL or BM after E15.5, suggesting that Notch1 is critical for definitive hematopoiesis (Hadland 2004). Finally, immunostaining for Notch1 in E9.5 and E10.5 mouse embryos showed that Notch1 is expressed in the ventral wall of the DA, and even in what appears to be some hematopoietic clusters (Robert-Moreno 2005). KO mice deficient in the transcription factor bound to activated Notch 1 - which is required for Notchl-specific transcriptional programs - were deficient in Runxl, Scl, and Gata2 ${ }^{+}$hematopoietic clusters in the E9.5 DA (Robert-Moreno 2005). This suggests that Notch1 is required for both definitive hematopoiesis and cluster formation in the AGM.

Subsequent efforts were focused on solidifying the hemogenic origin of DA clusters, as well as proving they contained dHSCs. In a groundbreaking study, North et al. used a Runxl reporter mouse to isolate $R u n x I^{+}$cells from the AGM and vitelline/umbilical arteries of embryos and found that at 10.5 , only the Run $x I^{+}$cells contained long-term multilineage repopulating cells, albeit at low levels of reconstitution (North 2002). DA clusters were Runx $I^{+}$at E11.5, and the majority were CD45-, although some cells were $\mathrm{CD} 5^{+}$(North 2002). Interestingly, at E10.5, all long-term repopulating cells (dHSCs) from the AGM and vitelline/umbilical arteries were CD45, whereas by E11.5 CD45 ${ }^{+}$and CD45- Runx $1^{+}$cells contained dHSCs (North 2002). The authors then decided to assess endothelial marker expression in the Runx $I^{+}$DA clusters, and found that some, but not all, Runx $I^{+}$cells expressed endothelial markers at E10.5 and E11.5, while a portion of cells express both endothelial and hematopoietic lineage markers or neither (North 2002). They assumed that the RunxI $I^{+} \mathrm{CD} 45^{-}$Endothelial ${ }^{-}$cells were mesenchyme. Upon transplantation of several tissues with varying degrees of hematopoietic and endothelial marker expression, they found that VE-Cadherin and CD45 in combination with Runxl were the best markers to delineate dHSCs in the E10.5E11.5 DA (North 2002). At E10.5, only RunxI ${ }^{+} \mathrm{CD} 45^{-} \mathrm{VE}-\mathrm{Cadherin}^{-}$cells appeared to contain HSCs, and by E11.5, Runx $1^{+} \mathrm{CD} 45^{+/}{ }^{-\mathrm{VE}-\mathrm{Cadherin}}{ }^{+/}$cells all contained dHSCs (North 2002). They also found that adult markers of HSCs, namely Sca-1 and c-Kit, were expressed on dHSCs in the AGM by E11.5, with all dHSCs expressing c-Kit (North 2002). Because of the apparent co-expression of hematopoietic and endothelial markers in dHSCs of the mouse DA, this pivotal work strongly supported a Runxl-mediated endothelial origin of dHSCs from the ventral wall of the DA.

The work by North et al. was reproduced by Taoudi et al., as they found that the $\mathrm{CD}^{+} 5^{+} \mathrm{VE}-\mathrm{Cadherin}^{+}$fraction of E11.5 AGMs contained the majority of myeloid progenitor potential in methylcellulose assays, all of the hematopoietic progenitor potential in long-term culture initiating cell assays, and displayed no in vitro endothelial potential (Taoudi 2005). They also showed that the E11.5 AGM CD45 ${ }^{+} \mathrm{VE}-\mathrm{Cadherin}{ }^{+}$ population contained all $\mathrm{dHSCs}$, and that this functional population could still be found in the E13.5 FL (Taoudi 2005) (which was later confirmed by Kim 2005). However, by E12.5 in the AGM, dHSCs were present in the VE-Caderin cell compartment, and VECadherin $^{+}$dHSCs were absent from the adult BM (Taoudi 2005). While it was possible to confirm the existence of dHSCs around the time of their generation, dHSCs in the $\mathrm{CD} 5^{+} \mathrm{VE}-\mathrm{Cadherin}^{+}$cell compartment are still extremely rare as determined by limiting dilution assays, with $\sim 1 \mathrm{dHSC} / \mathrm{AGM}$ at E11.5 (Taoudi 2005). This work was important 
for showing that dHSC in the E11.5 AGM express VE-Cadherin but are exclusively hematopoietic cells that contain the dHSC.

Both the North and Taoudi studies strongly suggested an endothelial origin of dHSCs in the mouse embryo and two subsequent studies attempted to validate this hypothesis. In 2008, Zovein et al. used a tamoxifen inducible VE-Cadherin-CreERT2 mouse crossed to a $\beta$-gal reporter mouse and induced labelling of $\mathrm{VE}$-Cadherin ${ }^{+}$cells during dHSC formation in the mouse embryo (Zovein 2008). They claimed that all definitive hematopoiesis derives from $\mathrm{VE}$-Cadherin ${ }^{+}$endothelial cells by observing $\mathrm{VE}$ Cadherin $^{+}$progeny in all blood cell types (including HSCs) of the FL and adult BM. A year later, Chen et al created a VE-Cadherin-Cre mouse and crossed it to Runx $1^{\mathrm{fl} / \mathrm{fl}}$ mice to show that Runx 1 expression in $\mathrm{VE}$-Cadherin ${ }^{+}$cells is required for dHSC formation (Chen 2009). VE-Cadherin-Cre ${ }^{+}$Runx $I^{\text {floxed }}$ mice showed a reduction in c- $\mathrm{Kit}^{+}$cells in the E11.5 FL, reduced CFU potential in E11.5 FL and AGM, harbored no dHSC activity via transplantation in the E11.5 AGM, and did not form DA clusters (Chen 2009). In contrast, they also created a Vav1-Cre mouse (which is supposed to be restricted to expression in the hematopoietic lineage) and showed that Runxl expression is not necessary in hematopoietic cells for dHSC formation, and attributed this to the conclusion that all dHSCs arise from an endothelial origin (Chen 2009). Three labs simultaneously confirmed that dHSCs specifically arise from the ventral wall of the dorsal aorta using time-lapse (Bertrand 2010) and live imaging (Kissa 2010) in zebrafish embryos, as well as live imaging in E10.5 mouse embryos (Boisset 2010), solidifying the concept that dHSCs arise from a hemogenic precursor in the ventral wall of the DA (Figure 1-6).

Interestingly, while the dHSCs in the CD $45^{+} \mathrm{VE}-\mathrm{Cadherin}{ }^{+}$compartment of the E11.5 are extremely rare (Taoudi 2005), the number of c-Kit ${ }^{+}$clustered cells in the DA number $\sim 700$ around the same time (Yokomizo 2010). To address this issue, Solaimani Kartalaei et al. showed that the majority of the c-Kit ${ }^{+}$cells in the E11 AGM are contained in a Sca-1 ${ }^{-}$compartment, and likely represent hematopoietic progenitors, not dHSCs (Solaimani Kartalaei 2015).

Together, these studies solidified the concept of dHSC generation from an endothelial origin (Figure 1-6) while also rapidly expanding the techniques needed for proper visualization of DA clusters. Over the next decade, several studies focused on the use of single cell sequencing technologies to further understand the transition from the DA ventral endothelium to dHSCs, and several "pro" and "pre" HSC subtypes were characterized that represented semi-discrete stages of dHSC generation from hemogenic endothelium (Rybtsov 2011, Gordon-Keylock 2013, Swiers 2013, Liakhovitskaia 2014, Rybtsov 2014, Boisset 2015). 

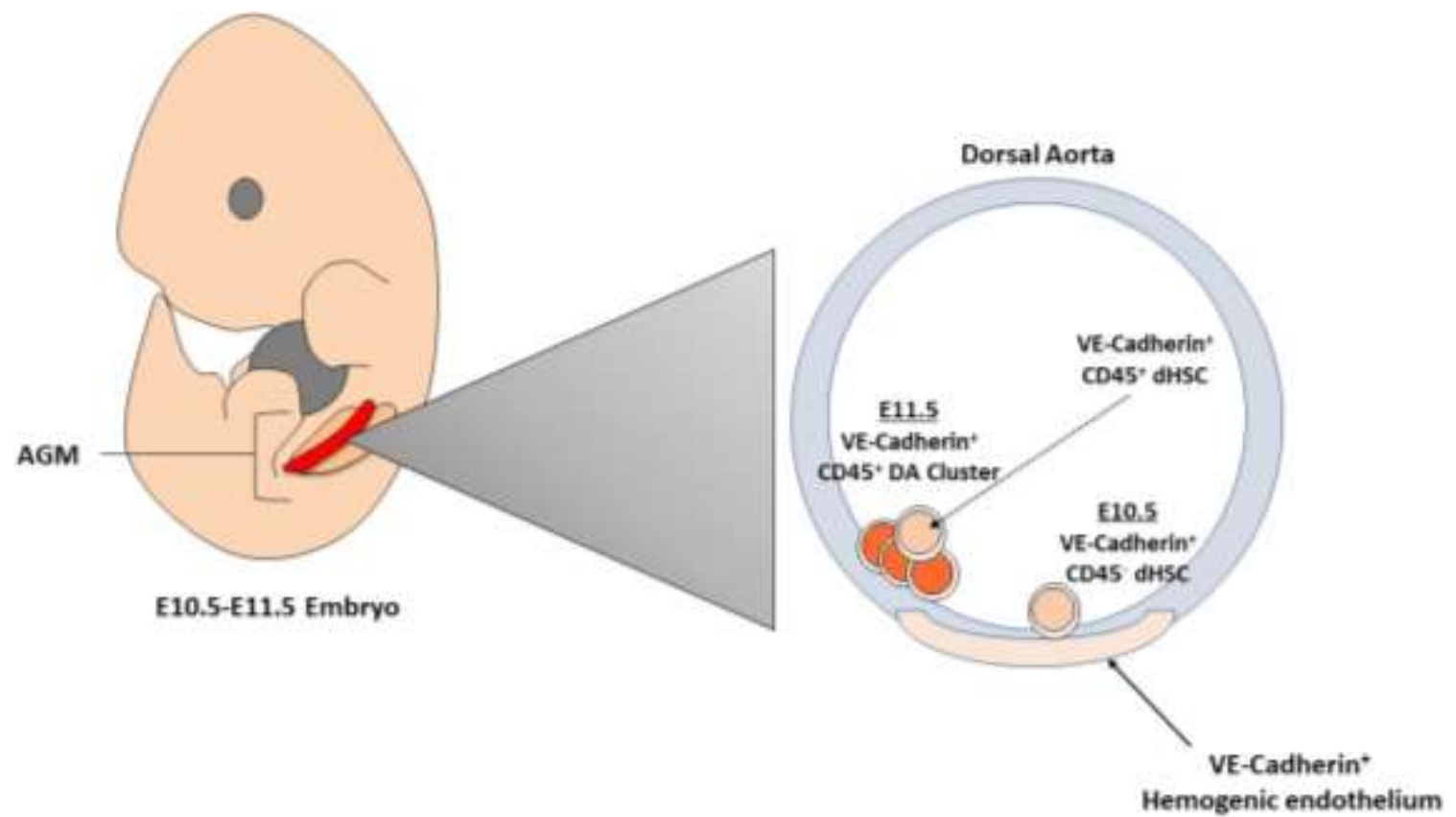

Figure 1-6. The definitive wave of hematopoiesis

The definitive wave of hematopoiesis and generation of definitive HSCs (dHSCs) occurs at E10.5 in the mouse embryo, with dHSCs being specified from the hemogenic endothelium of the ventral wall of the dorsal aorta (DA). 


\section{Is the dHSC the foundational hematopoietic cell for steady state hematopoiesis?}

While HSCT was instrumental in identifying and standardizing the definition of a bona fide HSC, it has recently come into question for its ability to identify the true progenitors and contributors to lifelong hematopoiesis during steady state. One of the first challenges to HSCT being the gold-standard assay for HSC identification was performed by the lab of Fernando Camargo (Sun 2014). Here, they developed a method to track clonal contributions of hematopoietic stem and progenitor cells to in vivo blood production in adult mice. These mice harbored a hyperactive Sleeping Beauty (HSB) transposase, a doxycycline-dependent transcriptional activator (M2) of the HSB, and a non-mutagenic transposon on three different alleles (Sun 2014). Doxycycline administration facilitated the mobilization of the transposon to a random site in the genome, as well as dsRed expression after the excision of the transposon. Therefore, doxycycline removal results in random, stably tagged and fluorescently labelled clones (Sun 2014). The contribution of these clones to blood production could then be assayed by DNA-sequencing. Interestingly, the Camargo lab found that steady-state hematopoiesis is maintained by thousands of clones that individually show minor contribution to mature blood lineages, and these clones do not appear to be progenitors downstream of HSCs (Sun 2014). This study seriously challenged the notion that a small number of HSCs were the sole contributors to native hematopoiesis, which was inferred based on transplantation assays. In the following years, several groups utilized in vivo clonal tracking techniques to challenge Sun 2014 (Sawai 2016, Chapple 2018, Sawen 2018). Therefore, there is still debate about the contribution of classically defined HSCs to native hematopoiesis.

Our laboratory recently used a multicolored reporter allele to lineage trace the contribution of specific murine embryonic tissues to lifelong blood production (Ganuza 2017). We crossed Flk1-, VE-Cadherin-, and Vav1-Cre lines with the Cre-inducible Confetti allele (Snippert 2010) to label cells of mesodermal (Flk1, E7.5), endothelial (VE-Cadherin, $\sim \mathrm{E} 8.5$-E10.5), or hematopoietic (Vav1, $\sim \mathrm{E} 10.5$-adult) origin in the embryo, and noted their contribution to adult hematopoiesis. (Ganuza 2017). Importantly, we were able to use the sample-to-sample variance in the distribution of Confetti fluorescent markers to quantify the number of initiating events, allowing us to estimate the number of precursors that give rise to lifelong murine hematopoiesis (Ganuza 2017). We found that the hematopoietic system is initiated by $\sim 600$ precursors, regardless of the Cre employed, which far exceeds the $\sim 1-2$ dHSCs previously thought to arise in the E10.5 AGM, and suggests that the assay employed to temporally determine the number and/or presence of true HSCs in the mouse embryo may be biased for a subset of transplantable HSCs (Ganuza 2017). Using a similar system, (Henniger 2017) the same phenomenon was observed in zebrafish, finding that $20-30$ progenitors of lifelong hematopoiesis form around the time of HSC emergence. 


\section{Summary}

Many decades of work uncovered the existence of several waves of embryonic hematopoiesis: two "primitive" waves mostly characterized by blood production of transient unipotent or multipotent progenitor cells in the YS, followed by a "definitive" wave of dHSCs that eventually seed adult tissues and provide lifelong blood production (Figure 1-7). Both waves appear to be generated from an endothelial cell with hematopoietic potential that arises from specified mesoderm: the hemangioblast in primitive hematopoiesis and the hemogenic endothelium in the dorsal aorta in definitive hematopoiesis. It is critical to note that HSCs do not immediately home to their resting place in the BM after specification in the AGM. HSCs journey throughout the mammalian embryo and are exposed to a variety of niches before finally colonizing and maintaining their presence in the adult BM.

\section{The HSC Niche Across Development}

The final destination of HSCs in mammals is the BM, where they are thought to reside for the remainder of the life of an individual. However, as mentioned above, their contribution to steady-state hematopoiesis is still under debate. Insights into the study of developmental hematopoiesis discovered that, while HSCs are specified in the dorsal aorta of the AGM region during development, they only stay in this niche for $\sim 1-2$ days before migrating to other tissues. In the murine system, HSCs migrate from the AGM to the FL around E12.5, where they are thought to undergo an expansion and then migrate to the fetal spleen and BM shortly after (Medvinsky 2011). It is also thought that HSCs migrate to the placenta shortly after generation in the AGM, although the AGM as a migratory versus an origination niche is still debated. It was Schofield in 1978 who first conceptualized the existence of an HSC niche (Schofield 1978). He noticed that cells derived from CFU-S colonies could not reconstitute hematopoiesis indefinitely, while BM-derived cells could. He therefore hypothesized that when HSCs left the BM niche and resided in the spleen niche, they lost their "immortality" (Schofield 1978). Earlier, it was also described that long-term culture of hematopoietic cells was only sustainable in the presence of a layer of BM feeder cells (Dexter 1977). For decades, scientists have tried to uncover the mechanisms of HSC niche control, in part to find the critical factors needed for HSC generation and/or expansion in cell culture for clinical purposes (Crane 2017, Gao 2018). In the next section I will explore the developmental path HSCs embark on after specification in the AGM, followed by a closer look at the most well characterized HSC niche - the adult BM — and the most understudied HSC niche - the fetal BM (FBM).

\section{The placenta: A site for HSC generation?}

The origins of resident HSCs in the placenta are mired in controversy. Using a transgenic mouse in which only embryonic tissue was fluorescently labelled, the presence of fetal-derived multipotent myeloid colony forming cells (CFU-GEMM) were found in 


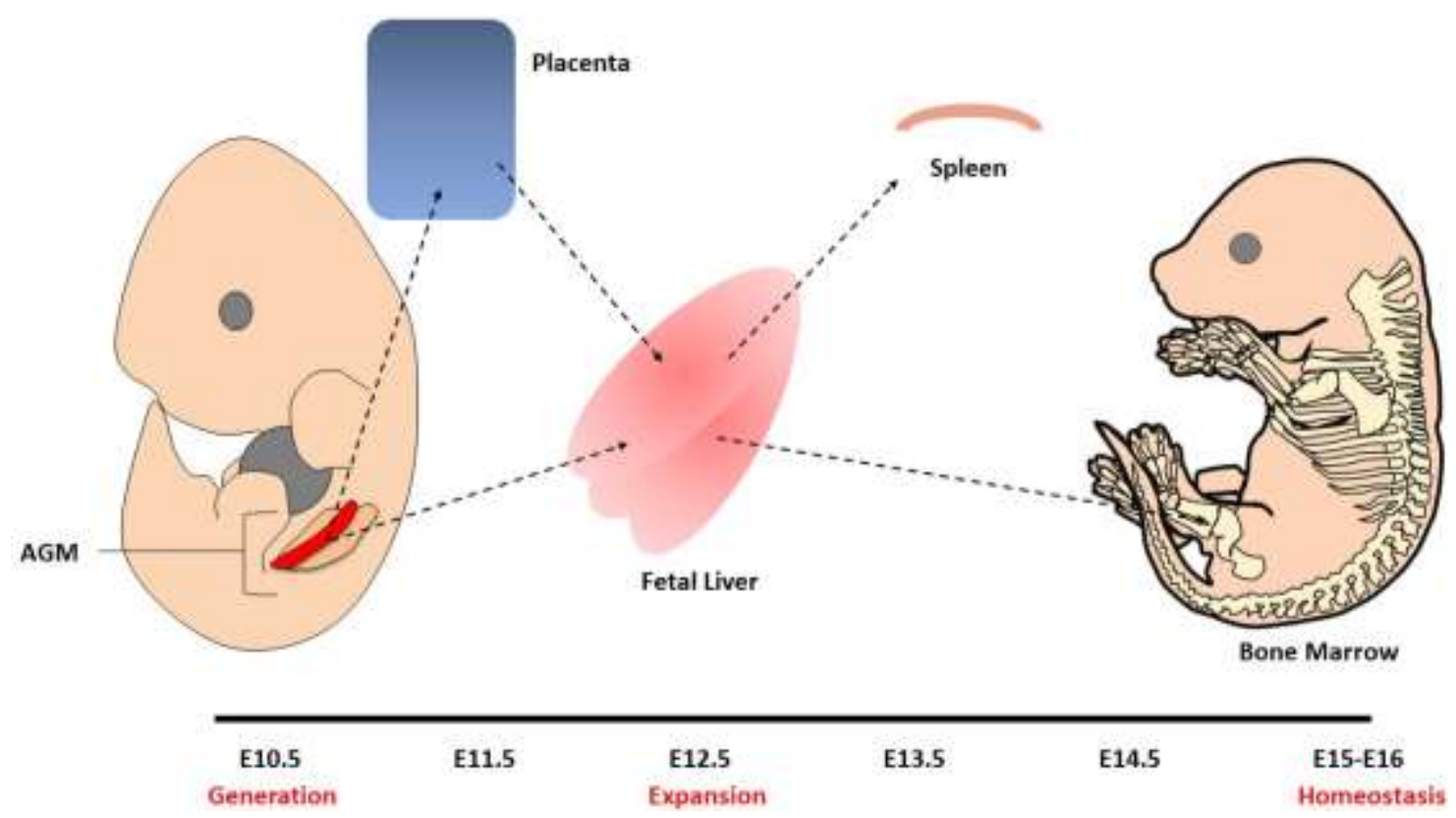

Figure 1-7. Developmental ontogeny of definitive hematopoiesis

HSCs are specified in the AGM and migrate to the fetal liver (FL) where they are thought to undergo a $\sim 30$-fold expansion. AGM-derived HSCs are also thought to seed the placenta shortly after generation. FL HSCs then seed the spleen and fetal BM during late ontogeny. HSCs will remain in the BM for the remainder of the life of an individual. 
the placenta as early as E9 (Alvarez-Silva 2003). While only a few CFU-GEMM were found at this time point ( $4-5)$, the number increased to $\sim 400$ by E10.5 - the same time that HSCs are thought to be specified in the AGM (Alvarez-Silva 2003). Interestingly, the authors also found the expression of key hematopoietic TFs such as Runx 1, Scl, and Tel/Etv6 in E9 placentas, suggesting that the placenta may be a site of HSC specification (Alvarez-Silva 2003). It should be noted that while HSCs can produce CFU-GEMM, it is not a bona fide test for HSCs. However, this discovery suggested that the placenta should be considered a major hematopoietic organ in mammals.

Two subsequent studies in 2005 determined that HSCs were present very early in the mouse placenta. Gekas et al. transplanted E10.5-E12.5 placenta and found that longterm reconstitution with donor contribution $>1 \%$ first occurred from E11 tissue, suggesting that bona fide HSCs are present at very early time points in the murine placenta (Gekas 2005). Via limiting dilution transplantation assays, they discovered that the numbers of HSC are very low at this time point, and peak to $50 \mathrm{HSCs}$ around E12.5, with a considerable drop-off until E15.5 at which point almost no HSC are present (Gekas 2005). The same year, Ottersbach et al. used a Ly-6A (Sca-1)-GFP transgene to find that all placental HSCs at E12 were Sca- $1^{+}$, which was shown previously for AGMderived HSCs (de Bruijn 2002) (Ottersbach 2005). They also found CD31/CD34 ${ }^{+} \mathrm{Sca}-1^{+}$ cells as early as E10 in the placenta, suggesting that HSC generation may be occurring de novo (Ottersbach 2005). It is important to note that while HSCs can be assayed in the E1 1 placenta, this is after both circulation and the proposed specification in the AGM, and no other tissues dissected at E10.5 or co-cultured in tissue explants have been known to generate HSCs (Medvinsky 2011).

The question of whether HSCs were specified de novo in the placenta led researchers to search for the presence of hemogenic endothelium in placental tissues. It was shown that placenta from E10-E11 Ncx $1^{-/-}$mice, which lack a heartbeat, contain $\mathrm{CD} 41^{+} \mathrm{CD} 31^{+}$hematopoietic precursors in the stromal vasculature of the placentaalthough $\mathrm{Ncxl}^{-/-}$mice did show lower numbers of these precursors, suggesting that the placenta may be colonized by HSCs as well as contain de novo generation potential (Rhodes 2008). Also, pre-circulation placenta tissue can produce all lineages in vitro after co-culturing with FBM stromal cells (OP9 and OP9-DL1) but cannot produce cells capable of reconstituting irradiated hosts (Rhodes 2008). Recently, a putative population of hemogenic endothelium (Prom $1{ }^{+} \mathrm{Sca}-1^{+} \mathrm{CD} 34^{+} \mathrm{CD} 45^{-}$, PS34 cells) was identified in the E10.5 AGM and subsequently decreased in frequency over 1-2 days (Pereira 2016). This population expressed hematopoietic and endothelial markers and displayed long-term lymphoid potential in immunodeficient mice after OP9-DL1 co-culture (Pereira 2016). Importantly, it was shown that PS34 cells were not maternal in origin via whole mount immunofluorescence (Pereira 2016). Together, these data suggest that the placenta appears to harbor hemogenic endothelial potential, but HSC generation is still unclear.

There is evidence that specific placental niche cells may regulate resident placental HSCs. Sasaki et al. identified prospective hematopoietic clusters in E10.5 murine placenta that were $\mathrm{CD} 31^{+} \mathrm{CD} 34^{+} \mathrm{c}-\mathrm{Kit}^{+}$via immunofluorescence, then used lasercapture microdissection to dissect surrounding tissues (Sasaki 2010). They found that the 
surrounding tissue was mainly endothelial and expressed high levels of $S c f$, suggesting that hematopoietic clusters of the AGM lie in an Scf-producing endothelial niche (Sasaki 2010). A similar model was confirmed in humans, as placenta-derived HSPCs displayed expansion and prolonged function when co-cultured with human umbilical vein cells (HUVECs), but not mesenchymal progenitors (Raynaud 2013).

Together, these studies show that HSCs exist and expand in the placenta early after HSC generation, and that their presence may be influenced by an $S c f$-dependent endothelial niche. Whether HSCs are generated de novo in the placenta remains unclear.

\section{The fetal liver: A niche for HSC expansion}

After several decades of research, the current model for HSC ontogeny after generation in the AGM is that HSCs migrate to the FL and subsequently undergo a dramatic expansion before seeding other tissues. In 1975, Johnson and colleagues isolated E9.5 FL and E11.5 FL and placed the tissues under the kidney capsule of irradiated adult hosts to see when the hematopoietic potential of the FL began (Johnson 1975). They observed no hematopoietic potential in E9.5 FL, but hematopoietic potential was observed from E11.5 FL, and they suggested that the FL did not have de novo HSC generating power on its own but was rather seeded by some other source (Johnson 1975). Their conclusions corresponded with what would subsequently be known about AGMspecific HSC generation. These results were confirmed in 1981 using mouse liver and avian embryos, but it was also shown that when "pre-migration" liver tissue was cultured in vitro with HSCs, FL tissue hematopoiesis could be achieved (Houssaint 1981). This helped solidify the hypothesis that FL hematopoiesis depends on the migration of HSCs from an exogenous source.

In the intervening years, much work was done to identify the FL niche as an expansion niche. Morrison compared the differences between FL and adult BM HSCs in terms of their ability to reconstitute an irradiated host, absolute frequency in the sorted HSC compartment, and cell cycle status (Morrison 1995). Through limiting dilution transplant assays, he found that the immunophenotypic HSC was highly enriched in the FL $(\sim 70 \%)$, and that $\sim 30 \%$ of the HSCs were cycling from E12.5-E15.5 (Morrison 1995). He also noted that, upon transplanting a similar dose of FL HSCs and adult BM HSCs, the FL HSCs showed a much greater repopulating ability via peripheral blood contribution than adult BM HSCs (Morrison 1995). Transplants competing FL and adult BM cells showed that FL outcompetes adult BM by 5-fold in terms of repopulating potential (Harrison 1997). This led the authors to conclude that the FL contained more HSCs than adult BM, but, as seen earlier (Morrison 1995), transplanting equal numbers of enriched HSCs still results in higher repopulating capacity of FL HSCs. Therefore, FL HSCs likely represent a more potent repopulating HSC pool in primary transplantation.

In an elegant and important study, Ema et al. performed a series of competitive repopulating limiting dilution transplantation assays with FL cells from E12-E18, and found that there was a massive expansion of HSC as determined by primary 
transplantation from E12-E16, followed by a subsequent decreased in HSC frequency from E16-E18 (Ema 2000). They determined that the number of FL HSC increased from $\sim 50$ to1500 from E12 to E16, respectively, a 30-fold increase (Ema 2000). They confirmed their results with a true limiting dilution assay, showing that there were $\sim 40$ and $1500 \mathrm{HSC}$ at E12 and E16, respectively. In a series of critical papers, Connie Eaves' group assayed the cycling potential of fetal HSCs compared to adult BM HSCs. In 2006, they showed that FL HSCs are primarily cycling, as upon injection of pregnant mothers with 5-fluorouracil — which preferentially affects cycling cells - no HSCs could be found at E14.5 or E18.5 from FL after transplantation (Bowie 2006). They also showed via transplantation assays that almost all HSCs in the E14.5 FL were in the G1 phase, whereas nearly all HSCs in the adult BM were in G0 (Bowie 2006). To confirm their results, they transplanted 10 HSCs into irradiated recipients from E14.5 FL and adult BM, then collected BM and performed limiting dilution analysis in secondary recipients to determine the cycling potential of HSCs in vivo (Bowie 2007). They reported that E14.5 FL HSCs displayed significant expansion 1-2 weeks post-transplant, but if the same number of E14.5 FL and adult HSC were isolated from primary recipients 6 weeks post-transplant and assessed for expansion capacity in secondary and tertiary recipients, the E14.5 FL HSCs appeared to have the same proliferative capacity as adult HSCs (Bowie 2007). This suggested that there are niche-specific factors that can control the regenerative capacity of HSCs, and that the adult BM niche appears to have the ability to induce a more quiescent phenotype.

To dissect the properties of the FL niche responsible for HSC maintenance, Wineman et al. isolated and immortalized $>200$ cell lines from E14.5 murine FL stroma and found a small subset of cell lines that, upon co-culturing with mouse BM, could maintain HSCs for 3 weeks in culture (Wineman 1996). They later characterized a single clone, AFT024, that could maintain mouse and human repopulation capacity from BM and FL enriched for LSK cells after 4-7 weeks of culture (Moore 1997a, Moore 1997b, Theimann 1998, Nolta 2002). It was also found that AFT024 secreted the Notch ligand, Dlk1, and that overexpression of Dlkl in stromal lines unsupportive of HSC maintenance enhanced their ability to maintain HSCs (Moore 1997b). A similar relationship to Notch signaling was observed in human FL stromal cell lines, which also were able to enhance HSC maintenance in vitro (Martin 2005). Another population of murine E15.5 FL cells, constituting less than $2 \%$ of FL cellularity, was shown to expand E15.5 LSKs by 2-fold after culturing for 3 days (Zhang 2004). Interestingly, these cells expressed a range of cytokines important for HSC maintenance, including Scf, Igf-2, Tpo, and Angptl2/3 (Zhang 2004). This population was further refined to be $S c f^{+} D l k^{+}$cells, and it was found that they are the main fetal stromal population that expresses Cxcl12, which is a homing factor for HSCs (Chou 2010). 2-3 weeks of co-culture of Scf $f^{+} \mathrm{Dk}^{+}$stroma with sorted HSCs resulted in a 20-fold increase in HSPCs and a slight increase in HSCs (Zhang 2004). More recently, the transcription factor and developmental regulator $\mathrm{Aft} 4$ was found to be critical for HSC migration to the FL via the reduction of HSC present in E12.5-E15.5 $\mathrm{Atf4}^{-/-}$mice (Zhao 2015). Importantly, they also isolated stromal and endothelial cells from Atf $^{-/-}$E15.5 FLs, co-cultured with E15.5 FL HSCs, and showed a reduction in repopulation capacity of HSCs co-cultured with KO cells verses WT cells (Zhao 2015). Therefore, several in vitro co-culture assays have demonstrated that the FL 
is an HSC-supportive microenvironment, although more work is needed to identify the critical factors needed for HSC expansion.

In 2016, Khan et al. provided an in vivo analysis of the FL HSC niche by utilizing a Nestin-GFP transgenic mouse, which labels endothelial cells and rare, Nestin ${ }^{+}$pericytes in the E14.5 FL (Khan 2016). They found that these pericytes surrounded endothelial cells on portal vessels of the FL via immunostaining, and they also discovered that $\mathrm{Nestin}^{+}$pericytes expressed $D l k l$ and were likely mesenchymal stem cells due to their ability to produce several stromal lineages in CFU assays (Khan 2016). Importantly, they also showed through whole-mount immunostaining that FL HSCs were in close proximity to Nestin ${ }^{+}$pericytes, and that depletion of these cells in vivo reduced HSC numbers (Khan 2016). Finally, they observed that the number of total niche cells in the FL expanded in correlation with the number of HSCs from E12.5-E14.5, which led them to hypothesize that it is an expansion of the niche and not a transcriptional change in the niche itself that allows for expansion of HSCs (Khan 2016). This is one of the only studies that critically assesses changes in the FL niche in vivo.

In summary, the FL is populated by HSCs around E12.5, appears to be a niche for expansion of HSCs from E12.5-E16.5, and the contribution of its niche cells to HSC expansion and maintenance still needs to be elucidated.

\section{The spleen: A limited source of HSCs}

The spleen appears to be a temporary site for HSCs during late ontogeny. By performing CFU assays in methylcellulose and competitive transplants for spleen cells across ontogeny, Wolber et al. saw little CFU-GEMM potential at E18, with an increase from P2 to P14 (Wolber 2002). They also observed that at P2 and P4, HSCs were present in the mouse spleen, and splenectomies on neonates showed that CFU and transplant reconstitution potential were unaffected in other hematopoietic tissues like the BM and liver (Wolber 2002). In a more rigorous study, Christensen et al. assayed spleen for the presence of HSCs via a series of competitive transplants across late fetal development (E14.5-E17.5), and found that HSCs can be found in the spleen as early as E14.5, but that appreciable repopulation of irradiated recipients from 1 e.e. spleen does not happen until $\sim$ E16 (Christensen 2004). They were able to detect immunophenotype HSPCs (LSK cells) in the spleen at E17.5, albeit at very low levels. Interestingly, they also showed that transient progenitors could be found in the E14.5 spleen, as irradiated recipients displayed B-cell—but not myeloid — reconstitution at 4 weeks post-transplant (Christensen 2004).

When testing for differences between adult spleen and BM HSCs, it was found that there were fewer spleen HSCs via immunophenotype and competitive transplantation, yet transplanting the same number of immunophenotypic HSCs from each tissue showed that HSC repopulating ability appeared to be similar between spleen and BM HSCs (Morita 2011). Adult spleen HSCs can even be secondarily transplanted, 
and spleen HSCs are twice as likely to be cycling via an in vivo BrdU labelling assay (Morita 2011). Therefore, splenic HSCs have the ability to home to the BM niche.

Ara et al. assessed the homing ability of HSCs in chemokine receptor $\mathrm{Cxcl1} 2^{-/-}$

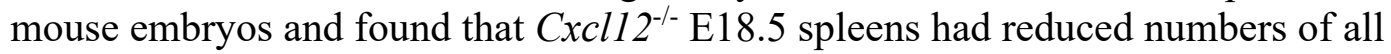
mature hematopoietic cells except Ter $119^{+}$blood cells, lower numbers of repopulating units as assayed by competitive transplants, and this reduced repopulating capacity could be rescued by enforced expression of Cxcl12 in Tie $2^{+}$endothelial cells (Ara 2003). This suggests that chemotaxis induced by $\mathrm{Cxcl} 12$ in response to the gradient of its ligand Cxcr4 is required for proper migration of HSCs into the spleen, and that $\mathrm{Tie} 2^{+}$endothelial cells may be responsible for production of Cxcl12 in the fetal spleen niche (Ara 2003).

To determine if fetal spleen could produce HSCs de novo, E13 murine spleens were maintained in explant culture for 4 days to assess HSC development, with E13 FL explant cultures as a control (Bertrand 2006). No HSCs could be generated from E13 spleens via competitive transplantation assay (Bertrand 2006). The authors also isolated stromal cell lines from E14.5 spleen to test the ability of the spleen microenvironment to maintain and/or expand HSCs, and plating FL HSCs on to the fetal spleen stroma resulted in a marked expansion and maintenance of macrophages, but not HSPCs (Bertrand 2006).

Together, these studies help to define the spleen as a temporary destination of fetal HSCs during late ontogeny in the mouse. Homing of HSCs to the fetal spleen appears to be dependent on Cxcl12 production in the spleen, and the splenic niche during gestation does not seem to be sufficient for HSC generation, maintenance, or expansion.

\section{The complexity of the adult bone marrow niche}

It was the work of Lambertson and Weiss in 1984 that helped determine the way we conceptualize the adult BM niche. They showed via colony forming assays that the distinct organization of the adult murine BM provided several "zones" for progenitor cells, and that progenitors could behave differently depending on the zone they resided in. By sub-fractioning the BM space, they determined that the greatest progenitor potential was near the endosteal surface of the bone, as well as near the central marrow (Lambertson 1984). They also proposed the existence of four niches: 1.) the endosteal zone, encompassing $0-0.02 \mathrm{~mm}$ of space from the bone surface, 2 .) the subendosteal zone, encompassing $0.02-0.08 \mathrm{~mm}$ of space from the bone surface, 3 .) the central zone, encompassing the cylindrical space in the center of the marrow surrounding the central sinus vein and extending throughout the length of the bone, with a radius of $0.1 \mathrm{~mm}$, and 4.) the intermediate zone, encompassing the space between the subendosteal and central zones (Lamberston 1984). In the intervening years researchers have incorporated other niches consisting of arteriolar, sinusoidal, and perivascular zones, with arteriolar/sinusoidal niches located in the central/intermediate zone and perivascular niches located throughout the BM space (Crane 2017). Therefore, the BM niche is an incredibly heterogenous space, and many efforts have been focused on identifying the niche cells and factors responsible for HSC and HSPC maintenance. 
The osteolineage. Osteoblasts were some of the first adult BM niche components to be identified as critical for HSC regulation. In the mid-90s, Taichman and Emerson first proposed the existence of an osteoblastic HSC niche (Taichman 1994, Taichman 1996). In 1994, they isolated osteoblasts from the endosteal region of human trabecular bone and co-cultured with human $\mathrm{CD} 34^{+}$hematopoietic progenitor cells, finding that this co-culture expanded numbers of $\mathrm{CD} 34^{+}$cells, but did not test for retention of function (Taichman 1994). Interestingly, they showed that granulocyte-colony stimulating factor (G-CSF) neutralization in these cultures diminished this expansion, suggesting that osteoblasts may act on CD34 ${ }^{+}$cells via a G-CSF mechanism (Taichman 1994). Two years later, they reported the use of functional assays on $\mathrm{CD}_{3} 4^{+}$cells to confirm that coculture with osteoblasts expands $\mathrm{CD}_{3} 4^{+}$cells for 2 weeks in vitro, and they observed an increase in CFUs and LTC-ICs from CD34 ${ }^{+}$cells cultured on osteoblast monolayers versus mixed BM stroma co-cultures.

By knocking-out the bone morphogenic receptor type IA (Bmprla), Zhang et al. noticed an increase in the presence of spindle-shaped $\mathrm{N}$-Cadherin ${ }^{+} \mathrm{CD}_{4} 5^{-}$osteoblasts (SNOs) in the endosteum of mouse bones, particularly in the trabecular sections (Zhang 2003). They also noticed that, although Bmpr $1 a^{-/-}$mice had reduced BM cellularity, the number of HSCs increased by two-fold, and they appeared to be in close proximity to SNOs via immunostaining (Zhang 2003). Following these observations, Calvi et al. activated osteoblasts therapeutically in transgenic mice and showed an increase in trabecular bone formation, trabecular bone-associated osteoblasts, and an increase in the number of LSK and HSC cells via LTC-IC and transplantation assays (Calvi 2003). They also noted that therapeutically activated osteoblasts produced enhanced levels of the Notch1 ligand Jagged1, and that inhibition of the Notch1 pathway in transgenic mice resulted in an ablation of the observed HSC expansion (Calvi 2003).

In a more recent study, therapeutically ablated osteoblasts in a transgenic mouse line resulted in a significant reduction in the capacity-but not the number - of BM HSCs to repopulate primary recipients, with an almost complete loss of secondarytransplantable HSCs (Bowers 2015). By transplanting fluorescently labelled HSCs into transgenic neonates with GFP-labelled osteolineage, others were able to identify distal and proximal osteolineage cells and profile them by scRNA-Seq (Silberstein 2016). They found that proximal osteolineage cells expressed higher levels of important HSC maintenance factors like Cxcl12 and Angpt1, but also expressed high levels of two new putative regulators: Angiogenin (Ang), a secreted RNase, and Embigin (Emb), an adhesion molecule (Silberstein 2016). They subsequently identified both factors to be critical for osteolineage regulation of HSC activity by knocking out the factors in osteolineage cells. Interestingly, knocking out Ang in osteolineage cells enhanced HSC cycling and decreased repopulation capacity, while mature $\mathrm{Ang}^{-/-}$osteoblasts only displayed lymphoid progenitor defects, suggesting different cells in the osteolineage interact with distinct HSPCs (Silberstein 2016). The same group later found that Ang expression in osteolineage cells has specific roles for HSCs and myeloid progenitors, facilitating quiescence of HSCs while simultaneously working to increase expansion of myeloid progenitors (Goncalves 2016). 
Together, these studies identify osteoblasts and cells of the osteolineage, which are exclusively found in the endosteal BM and are enriched in the trabecular bone, as critical of murine and human HSPCs maintenance.

The BM vasculature. The endothelial network in the adult $\mathrm{BM}$ is critical for proper maintenance of HSC function, primarily as a scaffold for other niche cell types. For instance, Tie $2^{+} \mathrm{BM}$ endothelial cells express low levels of $\mathrm{Cxcl12}$, and selective deletion of $\mathrm{Cxcl12}$ in $\mathrm{Tie}^{+}$cells results in a slight decrease in the repopulating capacity of HSCs but has no appreciable effects on HSC numbers or cell cycle status (Greenbaum 2013). Therefore, Cxcl12-expressing endothelial cells are not a critical component of the HSC niche. However, BM endothelial cells do express stem cell factor ( $S c f)$, and selective deletion of $S c f$ in Tie $2^{+}$endothelial cells from mouse BM results in a significant decrease in the number of HSCs via competitive transplantation assays (Ding 2012). Therefore, $S c f$-expression in Tie $2^{+}$endothelial cells appears to be a more important factor for HSC maintenance. It was later discovered that arterial endothelial cells are the primary Scf-secreting endothelial cells in the mouse BM (Xu 2018), as selective deletion of $S c f$ in arterial endothelial cells reduced HSC numbers and repopulation efficient while depletion of $S c f$ in sinusoidal endothelial cells had little effect on HSC maintenance (Xu 2018).

To determine the ability of BM endothelial cells to maintain HSPCs ex vivo, Butler et al. co-cultured mouse BM derived endothelial cells with HSCs and observed an expansion of the LSK compartment, along with a retention of CFU and long-term repopulating function via transplantation after 28 days of culture (Butler 2010). They then determined that secretion of Notch ligands by the endothelial cells in the co-culture system were responsible for HSC maintenance, as using endothelial cells from Notch1/Notch2 double KO mice abrogated this effect (Butler 2010). Interestingly, they also found that HSCs in which the Notch signaling pathway was activated were in close physical contact with sinusoidal endothelial cells in the mouse BM, suggesting that sinusoidal endothelial cells are a niche for Notch1/2 activated HSCs (Butler 2010). This was further confirmed by observing that selective deletion of the Notch1 ligand Jagged-1 in BM endothelial cells resulted in a 3-fold decrease in BM HSCs via limiting dilution transplant assays (Butler 2010). Several other in vitro studies have confirmed that coculturing murine and human HSPCs with endothelial cells fosters varying levels of expansion and maintenance (Butler 2012, Raynaud 2013, Sandler 2014, Gori 2017, Lis 2017). However, extrapolating these results to BM-derived endothelial cells requires caution, as these co-culture systems were developed using induced pluripotent stem cells (iPSCs) or HUVECs.

Together, these studies showed that BM endothelial cells can be a critical source of Scf and Notch-ligands for the maintenance of HSCs, and sinusoidal and arterial endothelial cells may constitute specific HSC niches. However, the effect of selective depletion of secreted factors in BM endothelial cells does not seem to have a strong effect on HSC maintenance, suggesting that they may be more important as a scaffold for other cell types. 
The sympathetic nervous system: Non-myelinating Schwann cells. One of the first studies to show that the sympathetic nervous system was important for HSPC maintenance in the BM was performed by Katayama and colleagues. They noted that a compound known to mobilize HSPC from BM was similar in structure to other compounds naturally synthesized in mammalian cells by an enzyme found in oligodendrocytes and Schwann cells (Katayama 2006). They observed that deletion of this compound in mice caused a failure of HSPCs to respond to mobilization agents such as G-CSF and was not due to reduced levels of the BM niche retention and homing factor, Cxcl12 (Katayama 2006). Later, it was observed that circulating HSCs are most prevalent in the bloodstream of mice 5 hours after exposure to light and are least prevalent after 5 hours in darkness, suggesting a circadian rhythm to HSC retention and mobilization (Mendez-Ferrer 2008). This was due to adrenergic signals sent to stromal cells from nerves in the BM, which facilitated downregulation of Cxcl12 (Mendez-Ferrer 2008). Yamazaki et al. observed that $T g f b r 2^{-/-}$HSCs in mice had reduced repopulating capacity, as well as increased proliferation in the BM (Yamazaki 2011). They therefore searched for Tgf $\beta$-producing cells in the BM niche, and found that non-myelinating Schwann cells, which form sheaths around nerves in the BM, are the primary source of BM Tgf $\beta$ (Yamazaki 2011). They also noted that HSCs were in close proximity to Schwann cells, and surgical denervation of the BM resulting in a decrease in transplantable HSCs (Yamazaki 2011). Together these studies suggest a role for the nervous system in BM HSC regulation, particularly in the Tgf $\beta$-signaling axis, although more studies are needed to elucidate the power of this niche.

Perivascular CAR cells. Perhaps some of the most interesting niche cells due to their tight association with HSC maintenance, perivascular cells wrap themselves around endothelial cells and are thought to exhibit properties of mesenchymal stem cells (MSCs). While many perivascular cells have been identified as important HSC regulators, they all express high levels of $\mathrm{Cxcl12}$ and are colloquially referred to as "Cxcl12-abundant reticular" (CAR) cells.

In 2010, Mendez-Ferrer et al. identified a rare population of $\mathrm{Nestin}^{+}$(typically expressed in nerve cells) MSCs that appeared to contain all of the CFU-Fibroblastic potential in the mouse BM and were in close proximity to both adrenergic nerve fibers and HSCs (Mendez-Ferrer 2010). Importantly, Nestin ${ }^{+}$MSCs expressed high levels of several HSC maintenance factors, including Scf, Cxcl12, and Angpt1, and depletion of $\mathrm{Nestin}^{+} \mathrm{MSC}$ in vivo reduced the LTC-IC potential of HSCs. Transplanted HSCs also appeared to home to $\mathrm{Nestin}^{+} \mathrm{MSC}$ post-transplantation (Mendez-Ferrer 2010). Together, these results identified Nestin ${ }^{+}$MSCs as a rare but important HSC niche component, and in the following decade many groups tried to identify similar cell types as well as factors expressed in these cells.

Morrison's group depleted $S c f$ from several niche cells and found that $S c f$ depletion from $\mathrm{Nestin}^{+}$cells had no effect on the function and frequency of HSCs, whereas $S c f$-depletion from a subset of perivascular cells that expressed the Leptin receptor $\left(\mathrm{Lepr}^{+}\right)$reduced immunophenotypic and functional HSC numbers (Ding 2012). 
Interestingly, they observed a preponderance of $\mathrm{Nestin}^{+}$cells around larger vessels in the BM, with a few Nestin ${ }^{+}$cells surrounding sinusoids. $S c f^{+}$cells were mostly concentrated around sinusoids, suggesting $\mathrm{Lepr}^{+}$cells are preferentially located around this vasculature in the BM (Ding 2012). Another group selectively deleted Cxcl12 in several BM niche cells and found that $\mathrm{Cxcl12}$ expression in another subset of perivascular cells that expressed Prx 1, a transcription factor important for limb bud development, was required for proper HSC maintenance (Greenbaum 2013). However, Cxcl12 expression appeared to be dispensable in Nestin ${ }^{+}$cells. Paul Frenette's lab identified a sub-population of $\mathrm{Nestin}^{+}$cells that also expressed the pericyte marker NG2 (Kunisaki 2013). These $N G 2^{+}$ pericytes expressed higher levels of Nestin and were closely associated with small arterioles, while other pericyte populations $\left(\mathrm{Nestin}^{\mathrm{lo}}\right.$ and $\left.\mathrm{Lepr}^{+}\right)$were associated with sinusoidal vessels (Kunisaki 2013). Through whole-mount imaging of murine BM, they showed that HSCs appeared to be in closer proximity to $\mathrm{NG}^{+}$cells compared to other perivascular cell types, and in vivo BrdU tracing showed that the $N G 2^{+}$-associated HSCs were highly quiescent (Kunisaki 2013). They also selectively depleted $N G 2^{+}$cells and showed that HSCs moved from the $\mathrm{NG}^{+}$arteriolar niche to the $\mathrm{Lepr}^{+}$sinusoidal niche and displayed increased cycling and reduced capacity for repopulating irradiated recipients (Kunisaki 2013). Together, these results suggested that $\mathrm{NG2}^{+}$pericytes and arterioles in the subendosteal BM constitute the niche for quiescent HSCs, and this was later supported by the finding that sinusoidal endothelium in the BM appears to facilitate trafficking of HSPCs and other blood types compared to the arteriolar niche, which may explain why quiescent HSCs are more closely associated with arterioles (Itkin 2016). Finally, in an attempt to dissect the different roles of Nestin ${ }^{+}$cells in the mouse BM, the Frenette group selectively deleted $S c f$ and $\mathrm{Cxcll} 2$ in $\mathrm{NG}^{+}$and $\mathrm{Lepr}^{+}$cells to determine the effects on HSC maintenance in the BM (Asada 2017). They found that Cxcl12 deletion in $\mathrm{NG}^{+}$cells but not $\mathrm{Lepr}^{+}$cells cause a reduction in HSC numbers and a relocalization of HSC cells away from arteriolar niches. In contrast, Scf deletion in $\mathrm{Lepr}^{+}$ cells but not arteriolar $\mathrm{NG}^{+}$cells caused a marked reduction in HSC numbers from the BM (Asada 2017).

Together, these studies highlight the heterogenous but critical roles of several perivascular CAR cell populations in maintaining HSC quiescence, BM retention, and repopulating capacity.

Adipocytes. Little is known about the role of adipocyte-specific HSC regulation within the adult BM niche. One study noted that different portions of murine vertebrae were populated with varying levels of adipose tissue (Naveiras 2009). In particular, the tail vertebrae showed enhanced numbers of adipocytes. Through transplantation assays it was discovered that the fatty BM of the tail contained fewer but more quiescent HSCs, and that HSPCs expand at a faster rate post-irradiation in "fatless" mice, suggesting that adipocytes regulate HSC cycling in the BM niche (Naveiras 2009). However, a later study showed that enhanced adipogenesis in the murine BM has no effect on HSC maintenance (Spindler 2014). Therefore, the role of adipocytes as regulators of HSCs in the BM niche is still unclear. 
Hematopoietic cells. While it is enticing to think of non-hematopoietic stroma as the sole regulator of $\mathrm{HSC}$ activity in the BM, hematopoietic cells have been shown to actively regulate BM HSCs.

Macrophages. Macrophages have been identified as critical BM-HSPC regulators. Winkler et al. reported that that upon granulocyte-colony stimulating factor (G-CSF) mobilization of HSCs, osteoblasts in the endosteal zone of mouse BM become depleted, as well as a population of macrophages that reside in the endosteum (osteomacs) (Winkler 2010). Upon depletion of osteomacs via transgenic mice or by chemical induction, HSCs were highly mobile and osteoblast depletion occurred in the endosteum, suggesting that osteomacs are a critical part of the endosteal HSC niche (Winkler 2010). At the same time, it was discovered that depletion of $\mathrm{CD} 169^{+}$but not CD169- macrophages in the mouse BM caused a concomitant reduction in Cxcl12, Scf, and other HSC maintenance factors in Nestin ${ }^{+} \mathrm{BM}$ cells, as well as enhanced mobilization of HSCs in response to G-CSF (Chow 2010). This suggested that multiple macrophage subtypes may affect different HSC niches. Selective depletion of BM macrophages has been shown to result in an expansion of HSCs in the murine BM (McCabe 2015). It has also been observed that experimental bacterial infections of mice increase macrophages and decrease the HSC pool, and depletion of macrophages after infection recovers $\mathrm{HSC}$ numbers, presumably through an IFN- $\gamma$-mediated pathway (McCabe 2015). Finally, transgenic mice in which only cells of the monocytic lineage express G-CSF receptor were treated with G-CSF and HSPC mobilization was still induced, suggesting that cells of the monocytic lineage in the murine BM are sufficient for HSPC mobilization (Christopher 2011).

Together, these studies identify BM-resident macrophages as an important mediator of HSC retention, but more work needs to be performed to determine if they affect other HSC properties

Megakaryocytes. As mentioned earlier, megakaryocytes and HSCs appear to be related in terms of particular cell surface markers, transcriptional programs, and differentiation pathways. Therefore, researchers have speculated mechanistic relationships between megakaryocytes and HSCs within the adult BM niche. By using 3D whole-mount imaging, the Frenette lab found that HSCs are often in close proximity to megakaryocytes, and that their spatial relationship to megakaryocytes does not come at the expense of their relationship to arteriolar niches (Bruns 2014). Incredibly, selective deletion of megakaryocytes in transgenic mice resulted in loss of HSC quiescence and an expansion of functional HSCs (Bruns 2014). They suggested that the megakaryocytic influence on HSCs is predominantly due to excretion of the chemokine, Cxc14, as BM megakaryocytes express $\mathrm{Cxcl} 4$ at high levels (Bruns 2014). Indeed, injection of Cxcl4 increased BM HSC quiescence, while $\mathrm{Cxcl}^{-/-}$mice displayed higher numbers of proliferating HSCs (Bruns 2014). Therefore, the Frenette lab has suggested a model in which megakaryocytes help maintain HSCs in the arteriolar niche. This model was supported by another study in 2014, which also suggested that BM HSC-associated 
megakaryocytes influence HSC quiescence via enhanced Tgfß signaling (Zhao 2014). Finally, another group in 2015 identified TPO production by megakaryocytes as another regulator of HSC quiescence in the BM niche (Nakamura-Ishizu 2015). Together, these studies highlight the role for megakaryocytes to potentially act on HSC quiescence in the arteriolar BM niche, although more work is needed to solidify megakaryocytes as critical HSC regulators.

Other hematopoietic cells. T-regulatory cells (T-regs) have been implicated in the protection of HSCs from immune responses (Fujisaki 2011). Through in vivo imaging of mouse BM, a population of endosteal T-regs that were closely situated next to HSPCs after transplantation were identified (Fujisaki 2011). Interestingly, depletion of T-regs in transgenic mice ablated the ability of HSCs to short-term engraft non-irradiated recipients, suggesting that T-regs may protect HSCs from acute immune responses (Fujisaki 2011).

Neutrophil clearance is the process by which specified neutrophils exit the BM to perform tissue-specific functions, then return to the BM cavity for destruction (CasanovaAcebes 2013). Effects of neutrophil clearance on the HSPC BM niche have been observed, as HSPCs appear to follow neutrophilic migration patterns: HSPCs are more likely to exit the BM during neutrophil circulation and more likely to be retained during neutrophil depletion via mobilization assays (Casanova-Acebas 2013).

Using a transgenic reporter mouse, Chen et al. identified a population of histidineproducing myeloid cells that were in close proximity to histidine-high, myeloid-biased HSCs (Chen 2017). Together, these histidine producing cells were also in close proximity to CAR cells (of undescribed type), and the authors showed that histidine production from adjacent myeloid cells was critical for the maintenance of histidine-high myeloidbiased HSCs (Chen 2017).

\section{The fetal bone marrow: An understudied but crucial niche in HSC ontogeny}

The adult BM is an incredibly complex niche for HSCs and contains a myriad of cells (Figure 1-8) that produce factors important for HSC retention, expansion, and functional integrity, the majority of which still need to be explored and potentially exploited for therapeutic purposes. Given the importance the adult BM as the final and major source of lifelong hematopoiesis, it is curious that the fetal BM (FBM) is such an understudied niche.

Some of the first assays testing for HSC and HSPC function in the FBM were by Wolber et al. in 2002. They determined that no appreciable amount of CFU-GEMM or HSC activity via transplantation could be observed in the BM until $\mathrm{P} 2$, with the number of CFU-GEMM exponentially increasing from P2 to P50+ (Wolber 2002). One year later, the Nagasawa group performed critical experiments to determine the mechanisms necessary for HSC migration to the FBM. Utilizing $\mathrm{CxCll}^{-/-}$mice, they reported that 


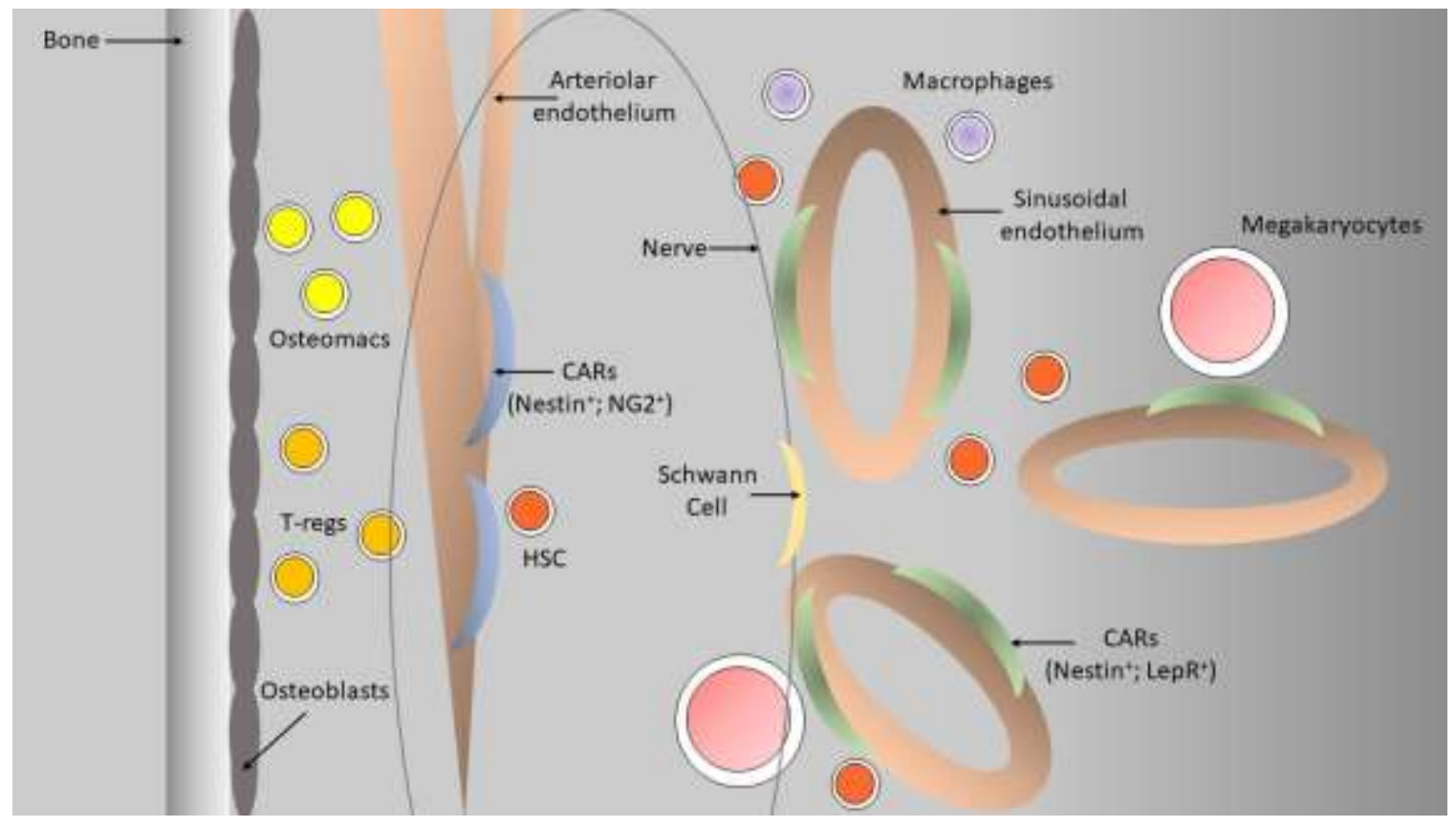

\section{Figure 1-8. The adult BM niche}

The adult BM cosists of a myriad of cell types that provide structural and humoral niches for HSCs. The endosteal niche is thought to be composed of osteoblasts, regulatory TCells (T-regs), and a subset of macrophages (Osteomacs). Quiescent HSCs are thought to be primarily located in arteriolar or sinusoidal niches within the central BM, and closely associate with CXCL12-abundant reticulocytes (CARs). 
Cxcl12 is required for HSC homing to the FBM at E18.5 (Ara 2003). Interestingly, they also showed that HSC appear to be accumulating in the peripheral blood (PB) of Cxcl12-1embryos at E18.5, suggesting proper release from the FL occurred, but HSCs could not enter the FBM space (Ara 2003). Importantly, enforced Cxcl12 expression in $\mathrm{Tie}^{+}$cells of $\mathrm{Cxcll} 12^{-/-}$mice recovered this homing defect, suggesting that $\mathrm{Tie}^{+}$endothelial cells are important for homing of FL HSCs to the FBM niche (Ara 2003). However, these results are still unclear due to the large increase in FBM HSCs from enforced Cxcl12 expression: $\sim 2$ HSCs in WT animals and $>10$ in rescue animals (Ara 2003). Nevertheless, this is one of the first hypotheses regarding the importance of niche components in the FBM influencing early HSC maintenance.

In another critical set of experiments, the Weismann group performed several competitive transplants of early FBM tissue to determine that the first HSCs colonize the FBM at E17.5, although they did note the presence of what appeared to be transient lymphoid progenitors in the FBM as early as E15.5 via the production of B cells in recipients at 4 weeks post-transplant (Chistensen 2004). It is important to note, however, that the authors were transplanting low numbers of cells: only the FBM from the long bones of the leg, and never more than 2 embryo equivalents. They also showed for the first time that immunophenotypic HSCs from the E14.5 FL could migrate in response to Cxcl12 and $S c f$ via a transwell migration assay, suggesting that production of these factors in the FBM niche may be important for migration from the FL to the FBM (Christensen 2004).

Connie Eaves' group identified an important developmental checkpoint that occurs in the BM. By treating pregnant mothers with 5-FU and then assaying tissues for number of HSCs via limiting dilution assay, they found that HSCs in the E18.5 FBM are primarily cycling (Bowie 2006). Their analyses suggested the presence of $\sim 10$ cycling HSCs in $10^{6} \mathrm{FBM}$ cells at E18.5. (Bowie 2006). Interestingly, they discovered that BM HSCs switch from a cycling to a predominantly quiescent phenotype at 3 weeks postbirth, as all HSC appear to be in the G1 fraction of E18.5 FBM and 3 week-old BM while nearly all HSCs are in the G0 fraction of 4 week-old BM (Bowie 2006). This was confirmed in a subsequent publication (Bowie 2007), suggesting that FBM HSCs are exclusively cycling, and do not become quiescent until $\sim 4$ weeks post-birth.

Karen Hirschi's lab has performed the best characterization of the FBM to date (Coşkun 2014) by dissecting the FBM niche in conjunction with the onset of HSC activity. They injected dissected fetal femurs with Dextran-FITC and noted that functional blood vessels were first formed in the diaphysis at E16.5, and they also noted that bone calcification first began in the diaphyseal regions of femurs at E16.5 via alizarin red staining (Coşkun 2014). Concomitant with vessel formation and calcification, CFU-GEMM could be found in the femur diaphysis at E16.5, but not in proximal or distal epiphysis until E17.5 (Coşkun 2014). FBM progenitors from E16.5-E18.5 showed greater cycling via Pyronin Y staining, and E16.5 FBM showed the first-albeit reduced - potential to repopulate irradiated recipients in a competitive transplant (Coşkun 2014). After noting that $\mathrm{Collal}^{+}$osteoblasts were present throughout the bone at E17.5, they hypothesized that osteolineage cells may be an important component of the FBM 
HSC niche. Therefore, they utilized Osterix ${ }^{-/-}$mice, which lack osteolineage cells, to test for HSPC and HSC function in the FBM. They observed that Osterix ${ }^{-/-}$E17.5 FBM did not contain transplantable HSCs, while Osterix $^{-/}$E17.5 FL appeared to be more highly enriched in repopulating cells than WT E17.5 FL (Coskin 2014). Interestingly, Osterix ${ }^{-/-}$ FL HSCs did not display homing defects, while FBM HSCs did. They performed qRTPCR on E17.5 FBM and saw upregulation of genes associated with vasculature and concluded that deletion of osteolineage cells from the FBM creates a more "endotheliallike" niche, which facilitates enhanced cycling and a decrease in homing (Coşkun 2014).

More recently, the Frenette lab hypothesized that the major remodeling of the FL portal vessel around birth and subsequent reduction in $\mathrm{Nestin}^{+}$pericytes - a proposed niche for FL HSCs - results in the egress of HSCs from the FL at this time, which correlates with the expansion of HSCs seen in the fetal/neonatal BM (Khan 2016). However, they used this observation to primarily make conclusions about the postnatal migration of FL HSCs to the BM, and since the FL portal vessel is still intact at early timepoints associated with FBM HSC activity (Khan 2016), its egress is probably not responsible for the seeding of HSCs seen in E16.5-E18.5 FBM.

Interestingly, the lab of Ralf Adams used confocal imaging of murine fetal femurs across development to identify the first formation of vasculature in the BM cavity at $\sim$ E15-15.5 (Langen 2017). This is one day earlier than observed by the Hirschi lab. The Adams lab also identified a new endothelial cell subtype in the fetal and early postnatal bone via CD31 and Endomucin (Emcn) expression coined "type E" endothelium (Langen 2017). Type E endothelium is the most abundant endothelial cell subtype at E16.5, but is virtually non-existent by P14, and appears to support Osterix ${ }^{+}$cells in the FBM (Langen 2017).

While these studies offer clues to the dynamics of hematopoiesis in the fetal and early neonatal BM, more work is needed to solidify the importance of this critical niche during hematopoietic development.

\section{The Present Study}

HSCs and their downstream progenitors are a heterogeneous population of cells that are indispensable for lifelong hematopoiesis and are often utilized in the clinic for the treatment of hematologic maladies via HSCT. Over several decades, it has been discovered that HSCs arise in the dorsal aorta of the developing embryo, migrate to the FL, and undergo a large expansion before reaching their final resting place in the BM (Figure 1-7). Many resources have been invested in understanding the roles of the different niches HSCs encounter along their journey, as well as the adult BM niche. A greater understanding of HSC niche regulation could provide clues for HSC maintenance and expansion in vitro. A critical niche during HSC ontogeny that has been greatly overlooked is the FBM. Current research asserts that the FBM is colonized by HSCs at E16.5 in a Cxcl12/Cxcr4-dependent manner (Ara 2003, Coşkun 2014). HSCs in the FBM appear to be predominantly cycling and continue to cycle until a shift toward quiescence 
at 4 weeks post-birth (Bowie 2006). It is still unknown which BM niche cells are important for HSC maintenance, or how the BM niche develops around birth to become the primary source of hematopoiesis in the adult. Furthermore, the immunophenotypic and functional assessment of the array of HSPCs known to be present in adult BM has yet to be performed in the FBM and early neonatal time points. For these reasons, we have meticulously characterized the hematopoietic progenitor compartment of the whole skeleton FBM from its colonization until after birth via competitive transplantation, immunophenotypic analysis of the HSPC compartment, functional assessment of specific progenitor populations, and single-cell RNA-sequencing of the hematopoietic and stromal FBM environment. We hypothesize that a thorough understanding of the hematopoietic potential and cellular composition of the FBM niche will be instrumental in revealing novel factors for HSC maintenance, expanding the available toolset for HSC-focused therapies. 


\section{CHAPTER 2. METHODS}

\section{Mice}

C57BL/6J (CD45.2), B6.SJL (CD45.1), and UBC-GFP ${ }^{+/ T}$ mice were purchased from The Jackson Laboratory and housed in a pathogen-free facility. Transplant recipient mice (F1 progeny of CD45.2 x CD45.1 crosses) were generated at St. Jude Children's Research Hospital. For all timed pregnancies, 1-2 female CD45.2 mice were placed with a single CD45.2 male overnight, followed by assessment for a vaginal plug before 10 a.m. the next morning. Positive females were separated, and embryos were designated embryonic day 0.5 (E0.5). For all animal experiments, special care was taken to follow the procedures approved by the St. Jude Children's Research Hospital Institutional Animal Care and Use Committee.

\section{Isolation of Hematopoietic and Stromal Cells}

To isolate hematopoietic cells in adult mice, tibias, femurs, pelvic bones, and spines were removed and bone marrow (BM) was released by crushing in ice cold phosphate buffered saline (PBS), followed by passage through $70 \mu \mathrm{m}$ filters. Cells were then resuspended in red blood cell lysis buffer for 5-10 minutes, washed with PBS/2\% fetal calf serum (FCS), and resuspended in PBS $/ 2 \%$ FCS. To isolate stromal cells in adult mice, crushed bone fragments were cut into small chips, combined with a portion of the released BM fraction, and placed in pre-warmed digestion media (PBS/2\% FCS $/ 0.4 \%$ collagenase II $/ 0.02 \%$ DNaseI) followed by gentle shaking (75RPM) at $37^{\circ} \mathrm{C}$ for 1 hour. After digestion, cells were passed through a $70 \mu \mathrm{m}$ filter and lysed for 5-10 minutes, washed with $\mathrm{PBS} / 2 \% \mathrm{FCS}$, and resuspended in $\mathrm{PBS} / 2 \% \mathrm{FCS}$.

Dissection of fetal tissues was performed under a dissection microscope in ice cold PBS. Briefly, fetal livers (FL) were removed and placed in ice cold PBS, followed by removal of all internal tissues and the skin. The fetal skeleton (except the bones of the head) was carefully removed and cleaned of any remaining tissues, washed in PBS, and placed in a dish of ice cold PBS for further processing. The isolation of hematopoietic and stromal cells from the fetal BM (FBM) followed the same protocol used for adult BM with the exclusion of the lysis step, as there was little trace of red blood cells. To isolate hematopoietic cells from FL, livers were gently crushed on a $70 \mu \mathrm{m}$ filter with the rubber end of a $1 \mathrm{ml}$ syringe while passing ice cold PBS over the tissue. Following this isolation step, FL hematopoietic cells were lysed, washed with $\mathrm{PBS} / 2 \% \mathrm{FCS}$, and resuspended in $\mathrm{PBS} / 2 \% \mathrm{FCS}$.

\section{Bone Marrow Transplantation}

For all experiments, recipient mice (CD45.2/CD45.1) were lethally irradiated with two doses of 5.8 Gy on the same day as transplantation. Donor (CD45.2) and support 
(CD45.1) BM cells were isolated and counted using a hemacytometer, with trypan blue used to assess viability. For whole BM (WBM) transplantation experiments, $1.5 \times 10^{6}$ donor WBM cells were combined with $2 \times 10^{5}$ support WBM cells and intravenously injected into the tail vein of recipient mice. Recipients were maintained on antibiotics for 10 days following injection. For transplantation of different bone sources, cells were isolated from separate bones from a single donor E15.5 embryo and the entire cell suspension was injected into the tail vein of a lethally irradiated recipient. For limiting cell transplants of UBC-GFP ${ }^{+/ T}$ multipotent progenitor 2 (MPP2) cells, 100 donor MPP2s were sorted into $1.5 \mathrm{ml}$ tubes containing PBS $/ 2 \% \mathrm{FCS}, 2 \times 10^{5}$ sorted support WBM cells were added, and the entire cell suspension was injected intravenously into the tail vein of recipient mice. All sorts were performed on a BD Aria cell sorter. For secondary transplantation, five aliquots of $5 \times 10^{6} \mathrm{WBM}$ cells were isolated from a single primary recipient and injected into the tail vein of five lethally irradiated secondary recipients.

\section{Peripheral Blood Analysis}

Transplant recipients were periodically assessed for donor cell contribution to the peripheral blood (PB). PB was collected from the retro-orbital plexus in heparinized capillary tubes, followed by lysis in red blood cell lysis buffer. Cells were then resuspended in PBS/2\% FCS and analyzed by flow cytometry for donor and lineage contribution on a BD LSR Fortessa. For WBM primary and secondary transplants and separate bone transplants, PB was stained with CD45.1-FITC, CD45.2-v500, [B220, CD11b, Gr-1]-PerCP-Cy5.5, and [B220, CD4, CD8]-PE-Cy7. The myeloid lineage was considered $\mathrm{CD} 11 \mathrm{~b}^{+} \mathrm{Gr}-1^{+}$, the B-cell lineage was considered $\mathrm{B} 220^{+}$, and the T-cell lineage was considered $\mathrm{CD} 4^{+} \mathrm{CD} 8^{+}$. For MPP2 limiting cell transplants, we also included a separate analysis of non-lysed PB stained with CD41-PerCPe710 (platelets) and Ter119-PECy7 (erythrocytes). A full list of antibodies can be found in Table 2-1. All data were analyzed using FlowJo version 10.

\section{Recipient BM Analysis}

At a terminal ( $>20$ weeks) timepoint after transplantation, recipients of WBM were euthanized and hematopoietic cells were isolated from the BM. Cells were assessed via flow cytometry on a BD LSR Fortessa for the presence of donor-derived common myeloid progenitors (CMP, Lineage ${ }^{-}-\mathrm{Kit}^{+} \mathrm{Sca}-1^{-} \mathrm{CD} 34^{+} \mathrm{CD} 32 / 16^{\mathrm{lo}}$ ), granulocyte/monocyte progenitors (GMP, Lineage ${ }^{-} \mathrm{c}-\mathrm{Kit}^{+} \mathrm{Sca}-1^{-} \mathrm{CD} 34^{+} \mathrm{CD} 32 / 16^{\mathrm{hi}}$ ), megakaryocyte/erythroid progenitors (MEP, Lineage ${ }^{-} \mathrm{c}-\mathrm{Kit}^{+} \mathrm{Sca}-1^{-} \mathrm{CD} 34^{-} \mathrm{CD} 32 / 16^{-}$), common lymphoid progenitors (CLP, Lineage $\left.{ }^{-c}-\mathrm{Kit}^{\mathrm{mid}} \mathrm{Sca}-{ }^{\mathrm{mid}} \mathrm{CD} 127^{+}\right)$, long-term hematopoietic stem cells (LT-HSC, Lineage ${ }^{-S c a}-1^{+} \mathrm{c}-\mathrm{Kit}^{+}$(LSK)Flt3 ${ }^{-} \mathrm{CD} 48^{-} \mathrm{CD} 150^{+}$), short-term HSCs (ST-HSC, LSKFlt3-CD48-CD150), MPP2s (LSKFlt3-CD48 ${ }^{-} \mathrm{CD} 150^{+}$), MPP3s (LSKF1t3 ${ }^{-} \mathrm{CD} 48^{+} \mathrm{CD} 150^{-}$), and MPP4s (LSKFlt3 ${ }^{+} \mathrm{CD} 48^{+} \mathrm{CD} 150^{-}$). In all cases, the Lineage $^{-}$fraction showed low expression of CD4, CD8, B220, Gr-1, and Ter119. 
Table 2-1. List of antibodies

\begin{tabular}{|c|c|c|}
\hline Antibody & Fluorophore & Clone \\
\hline B220 & BV605 & RA3-6B2 \\
\hline B220 & PE-Cy7 & RA3-6B2 \\
\hline B220 & PerCP-Сy5.5 & RA3-6B2 \\
\hline CD11b & PerCP-Сy5.5 & $\mathrm{M} 1 / 70$ \\
\hline CD127 & PE-Cy7 & A7R34 \\
\hline CD150 & PE-Cy7 & TC15-12F12.2 \\
\hline CD150-CITE-Seq & $\mathbf{N A}$ & TC15-12F12.2 \\
\hline CD184 (CXCR4) & FITC & L276F12 \\
\hline CD32/16 & A700 & 93 \\
\hline CD34 & A647 & RAM34 \\
\hline CD34 & FITC & RAM34 \\
\hline CD4 & BV605 & RM4-5 \\
\hline CD4 & PE-Cy7 & RM4-5 \\
\hline CD41 & PerCPe710 & eBioMWReg30 \\
\hline CD45 & $\mathrm{PE}$ & $30-F 11$ \\
\hline CD45.1 & FITC & A20 \\
\hline CD45.2 & v500 & 104 \\
\hline CD48 & A700 & HM48-1 \\
\hline CD48-CITE-Seq & $\mathbf{N A}$ & HM48-1 \\
\hline CD8 & BV605 & $53-6.7$ \\
\hline CD8 & PE-Cy7 & $53-6.7$ \\
\hline c-Kit & APC780 & $2 \mathrm{~B} 8$ \\
\hline Flt3 & APC & A2510 \\
\hline Flt3-CITE-Seq & NA & A 2510 \\
\hline Gr-1 & BV605 & RB6-8C5 \\
\hline Gr-1 & PerCP-Cy5.5 & RB6-8C5 \\
\hline Sca-1 & PerCP-Сy5.5 & E13-161.7 \\
\hline Sca-1-CITE-Seq & NA & E13-161.7 \\
\hline Ter119 & BV605 & TER119 \\
\hline Ter119 & PE-Cy7 & TER119 \\
\hline
\end{tabular}


A full list of antibodies can be found in Table 2-1. All data were analyzed using FlowJo version 10 .

\section{Analysis of Hematopoietic Stem and Progenitors Across Bone Marrow Development}

Liver and entire skeleton BM hematopoietic cells were isolated from E15.5postnatal day 0 (P0), P2, P4, P6, P8, P14, P21, and P28 CD45.2 mice and assessed via flow cytometry on a BD LSR Fortessa for the presence of LT-HSCs, ST-HSCs, MPP2s, MPP3s, and MPP4s. A full list of antibodies can be found in Table 2-1. All data were analyzed using FlowJo version 10.

\section{CXCR4 Cell-Surface Expression}

Hematopoietic cells from E15.5-P0 livers were isolated and assessed via flow cytometry on a BD LSR Fortessa for the presence of LT-HSCs, ST-HSCs, MPP2s, MPP3s, and MPP4s, as well as cell-surface Cxcr4. A full list of antibodies can be found in Table 2-1. All data were analyzed using FlowJo version 10.

\section{CXCL12 Migration Assays}

FL LT-HSCs, ST-HSCs, MPP2s, MPP3s, and MPP4s were sorted on a BD Aria cell sorter and dispensed into the top portion of a 24-well transwell plate containing StemSpan ${ }^{\mathrm{TM}}$ Serum-Free Expansion Medium (SFEM) with no supportive cytokines. Meanwhile, the bottom portion of each transwell contained either $0 \mathrm{ng} / \mathrm{ml}$ or $100 \mathrm{ng} / \mathrm{ml}$ soluble CXCL12 in SFEM. After a 5 hour incubation, the bottom portion of the transwell was collected and plated in 3ml M3434 Methocult to determine colony forming unit (CFU) potential. At the same time, a known number of sorted cells which were not placed in transwells were also plated into M3434 Methocult to determine the input CFU frequency. After 10-12 days, CFUs were counted, and the migration potential of each population was calculated using the following formula:

(\# of migratory CFU)/[(Frequency of input CFU) * (\# input cells used in transwell assay)]

\section{Single Cell CFU Assays}

Single MPP2s from E16.5-P0 FL/FBM and adult BM were sorted on a BD Aria cell sorter directly into 96-well plates containing 100ul of M3231 Methocult supplemented with $25 \mathrm{ng} / \mathrm{ml} \mathrm{SCF}, 25 \mathrm{ng} / \mathrm{ml}$ Flt3L, $25 \mathrm{ng} / \mathrm{ml} \mathrm{IL-11,} 10 \mathrm{ng} / \mathrm{ml} \mathrm{IL-3,} 10 \mathrm{ng} / \mathrm{ml}$ GM-CSF, 25ng/ml TPO, and 4U/ml EPO. After 10-12 days of culture, wells were scored for the presence and phenotype of CFUs. 


\section{Single Cell RNA Sequencing}

E16.5, E18.5, P0, and adult BM hematopoietic progenitors (CD45 ${ }^{+}$Lineage ${ }^{-c}-$ $\left.\mathrm{Kit}^{+}\right)$and stromal cells (CD45-Ter119-) were isolated as described above and sorted on a BD Aria cell sorter. Prior to sorting, hematopoietic and stromal cells were stained with a panel of CITE-Seq antibodies for downstream sequencing analyses. Post sort viability was determined, and samples with less than $80 \%$ viability were removed before generating sequencing libraries. mRNA expression libraries were constructed according to the Chromium single cell 3' reagent v3.1 protocol, while CITE-Seq expression libraries were constructed according to the protocol described in Stoeckius et al. (Stoeckius 2017). Both libraries were sequenced using Illumina technology.

\section{Analysis of Single Cell Data}

Following Illumina sequencing, the raw data was processed with the CellRanger pipeline provided by $10 \mathrm{x}$ Genomics. Count matrices were imported into $\mathrm{R}$ version 4.0 and all analysis was performed using the Seurat R package version 3.1.5 (Butler 2018). Clustering was performed according to the default instructions in the Seurat $\mathrm{R}$ package. To aid in the annotation of clusters, we used the FindAllMarkers function in Seurat to provide a list of gene expression comparisons among clusters, as well as the percentage of cells in each cluster with significant levels of gene expression. We also utilized ROCtest based statistics to define the key genetic markers in each cluster. Finally, we compared the gene expression patterns seen in our clusters to previously compiled single cell RNA-Sequencing datasets of adult BM stroma and hematopoietic cells (CabezasWallscheid 2014, Dahlin 2015, Kowalczyk 2015, Paul 2015, Wilson 2015, de Graf 2016, Dwyer 2016, Nestowara 2016, Cai 2017, Chhiba 2017, Velten 2017, Dahlin 2018, Giladi 2018, Rodriguez-Fraticelli 2018, Tusi 2018, Zheng 2018, Baccin 2019, Baryawno 2019, Mende 2019, Pellin 2019, Severe 2019, Tikhonova 2019, Wolock 2019). All UMAP and expression plots were generated using packages in R Version 4.0. 


\section{CHAPTER 3. RESULTS}

\section{Long-Term Repopulating Cells Can Be Found in E15.5 FBM}

HSCs are thought to be generated in the ventral wall of the dorsal aorta in the E10-E10.5 murine embryo, followed by migration to the FL where they undergo an expansion from E12-E15 (Medvinsky 2011). The BM is the final destination for mammalian HSCs, and their presence has been found as early as E16.5 in murine FBM (Coşkun 2014). Earlier groups identified E17.5 as the earliest time point for HSC arrival in the FBM, and E18.5 is a time point at which clear HSC activity can be seen (Ara 2003, Christensen 2004, Bowie 2006, Bowie 2007). However, attempts to identify the earliest FBM HSCs primarily utilized long bones (femur, tibia, hip) or did not state the source of BM collected (Ara 2003, Christensen 2004, Bowie 2006, Bowie 2007, Coşkun 2014). This allows for the possibility of HSCs colonizing the marrow space of other bones at similar or earlier time points. Indeed, there are conflicting reports of the first embryonic time point for vascularization within the BM cavity, with Coşkun et al. providing evidence for E16.5 vascularization (Coşkun 2014) and Langen et al. providing evidence for even earlier (E15-E15.5) (Langen 2017).

To thoroughly characterize the full HSC potential of the murine FBM, we isolated and competitively transplanted the BM from the entire skeleton of E15.5, E16.5, E17.5, E18.5, and P0 embryos and neonates (Figure 3-1). The embryonic skulls proved too technically challenging to properly isolate without excessive inclusion of other tissues and were excluded. We injected $1.5 \times 10^{6} \mathrm{CD} 45.2^{+}$donor BM cells with $2 \times 10^{5}$ supportive BM cells per lethally irradiated recipient from each time point, as E15.5 and E16.5 embryos contain $\sim 1.5 \times 10^{6}$ total BM cells (Figure 3-1). Therefore, recipients of E15.5 and E16.5 FBM received $\sim 1$ embryo equivalent (e.e.) of BM. We also injected adult BM into lethally irradiated recipients as a positive control, and all recipients were followed for at least 20 weeks for reconstitution of the hematopoietic system from donor cells in the peripheral blood (PB) (Figure 3-1). Donor chimerism $>1 \%$ was considered true repopulation (Figure 3-1).

As expected, $1.5 \times 10^{6}$ adult BM cells were sufficient for long-term repopulation in irradiated recipients, as $9 / 9$ recipients displayed $\sim 80 \%$ PB chimerism at 20 weeks posttransplant (Figure 3-2A). Inspection of lineage output (myeloid, B-lymphoid, Tlymphoid) from donor cells in the $\mathrm{PB}$ of each recipient of adult BM at 20 weeks posttransplant revealed a balanced distribution of lineages, suggesting that there were no enrichments for lineage-biased HSCs (Figure 3-2B). Recipients of P0 BM displayed similar levels of PB chimerism to adult BM recipients (Figure 3-3A) and all but one myeloid-biased recipient (14/15) had normal PB lineage distributions at 20 weeks posttransplant (Figure 3-3B). Therefore, P0 BM appears to contain a sufficient number of HSCs for long-term, balanced reconstitution of a lethally irradiated host.

Transplantation of $1.5 \times 10^{6} \mathrm{E} 18.5 \mathrm{FBM}$ cells resulted in long-term reconstitution of 14/14 recipients, although the levels of reconstitution in most recipients were lower 


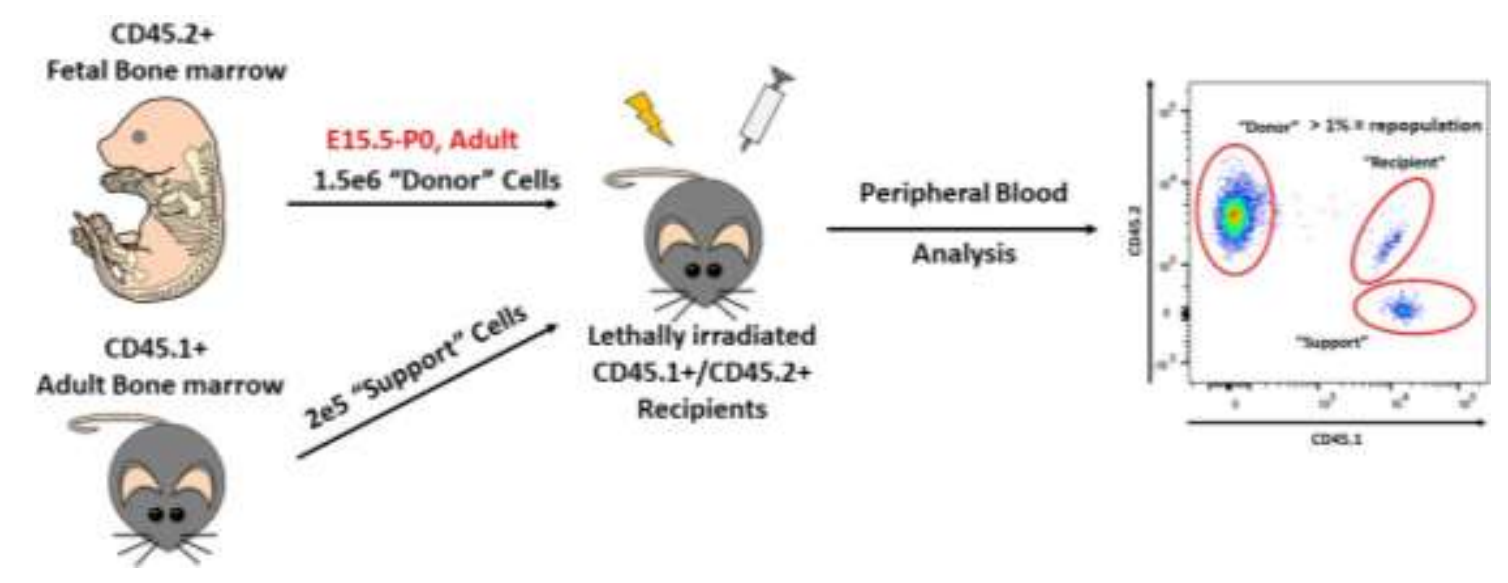

Figure 3-1. Primary transplant of FBM

$1.5 \times 10^{6} \mathrm{CD} 45.2^{+}$"donor" $\mathrm{BM}$ cells from E15.5-P0 and adult were transplanted into lethally irradiate CD45. $1^{+} / \mathrm{CD} 45.2^{+}$recipients, along with $2 \times 10^{5} \mathrm{CD} 45.1^{+}$"support" cells. Recipient peripheral blood (PB) was analyzed for donor contribution over a period of 20 weeks.
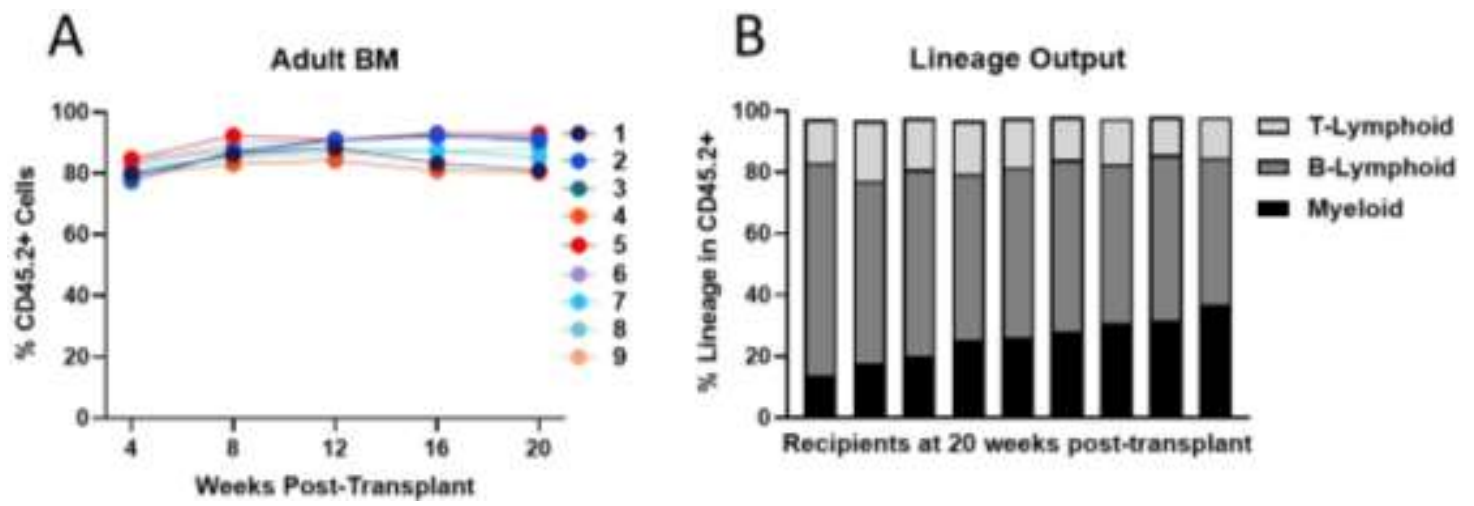

Figure 3-2. Primary transplantation of adult BM

A. Contribution of CD45.2 $2^{+}$donor adult $\mathrm{BM}$ to the $\mathrm{PB}$ of transplanted recipients over 20 weeks $(n=9)$. B. T-lymphoid, B-lymphoid, and myeloid lineage output within the CD45.2 $2^{+}$compartment of $\mathrm{PB}$ in recipients at 20 weeks post-transplant $(\mathrm{n}=9)$. 

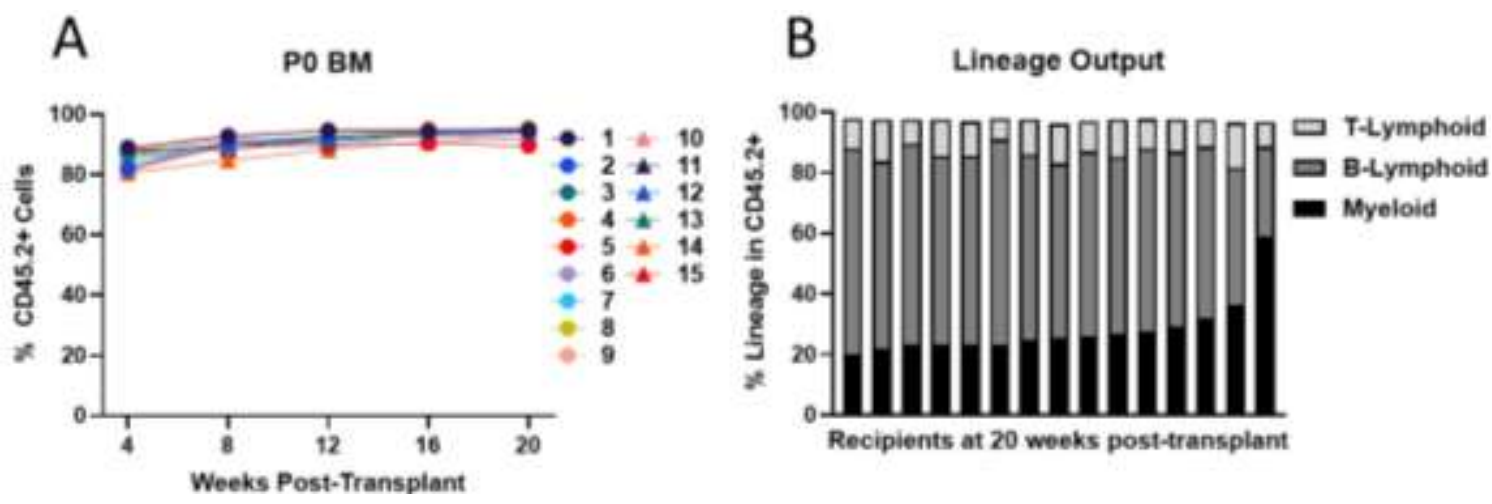

Figure 3-3. Primary transplantation of P0 BM

A. Contribution of CD45.2 $2^{+}$donor $\mathrm{P} 0 \mathrm{BM}$ to the $\mathrm{PB}$ of transplanted recipients over 20 weeks $(\mathrm{n}=15)$. B. T-lymphoid, B-lymphoid, and myeloid lineage output within the CD 45. $2^{+}$compartment of PB in recipients at 20 weeks post-transplant $(n=15)$. 
than from adult or P0 BM (Figure 3-4A). While all recipients displayed tri-lineage donor reconstitution at 20 weeks post-transplant, some recipients showed slight expansion of the myeloid or B-lineages, suggesting the engraftment or expansion of lineage-biased HSCs in these recipients (Figure 3-4B). Meanwhile, transplantation of E17.5 FBM resulted in even greater donor PB stochasticity in irradiated recipients, and while 19/19 recipients displayed donor repopulation $>1 \%$ at 20 weeks post-transplant, two recipients were only reconstituted at $1.1 \%$ and $1.3 \%$ (Figure 3-5A). Likewise, there was greater variability in the lineage reconstitution from donor cells in recipients, and several mice showed enhancement of either myeloid or B-cell lineages (Figure 3-5B).As expected, transplantation of $1.5 \times 10^{6} \mathrm{E} 16.5 \mathrm{FBM}$ cells resulted in a marked reduction in repopulating potential as only $8 / 14$ recipients displayed long-term repopulation $>1 \%$, which was quite variable among repopulated recipients (Figure 3-6A). These recipients also displayed variable donor lineage output, with $2 / 8$ long-term reconstituted mice showing 95\% and 96.6\% B-lineage output from donor cells (Figure 3-6B).

Finally, we were able to detect long-term reconstitution in $8 / 18$ recipients of $1.5 \mathrm{x}$ $10^{6}$ E15.5 FBM cells (Figure 3-7A). This is the first recorded demonstration of long-term repopulating cells within the E15.5 FBM via transplantation. Interestingly, many of the recipients with long-term reconstitution displayed donor chimerism $<10 \%$ at 4 weeks post-transplant, followed by a steady increase in donor contribution until 20 weeks (Figure 3-7A). This suggests a lack of functional hematopoietic progenitor cells in the E15.5 FBM compared to later BM time points, as progenitors have transient repopulation capacity and may preferentially contribute to early blood production post-transplant (Pietras 2015) (Figure 3-7A). Similar to E16.5 transplants, donor E15.5 long-term repopulating cells showed high variation in lineage production, as one recipient showed only B-lineage production at 20 weeks post-transplant (Figure 3-7B).

Together, these data show that the first long-term repopulating cells in the murine FBM are present at E15.5 - one day earlier than previously reported (Coşkun 2014) (Figure 3-7). Given that we were transplanting 1 e.e. into each recipient and only $50 \%$ of recipients displayed long-term repopulating potential (Figure 3-8), these long-term repopulating cells are still exceedingly rare. Interestingly, there were long-term repopulating cells that displayed uni- or bipotential lineage reconstitution from E15.5E17.5, suggesting the presence of highly lineage-biased or even "lineage-bypassing" HSCs (Yamamota 2013) at these time points (Figure 3-8). However, the presence of lineage biases at $\mathrm{P} 0$ and $\mathrm{E} 18.5$ coupled with the increasing stochasticity in donor PB chimerism from $\mathrm{P} 0$ to E15.5 suggests that these lineage-biased/bypassing HSCs are present at all timepoints, but their functional readout is amplified at earlier timepoints due to a reduction in total HSC numbers. Given that E18.5 is the first timepoint at which $100 \%$ of transplanted recipients show long-term tri-lineage repopulation, we conclude that E18.5 marks the initiation point of a BM space containing robust repopulation potential (Figure 3-8). 

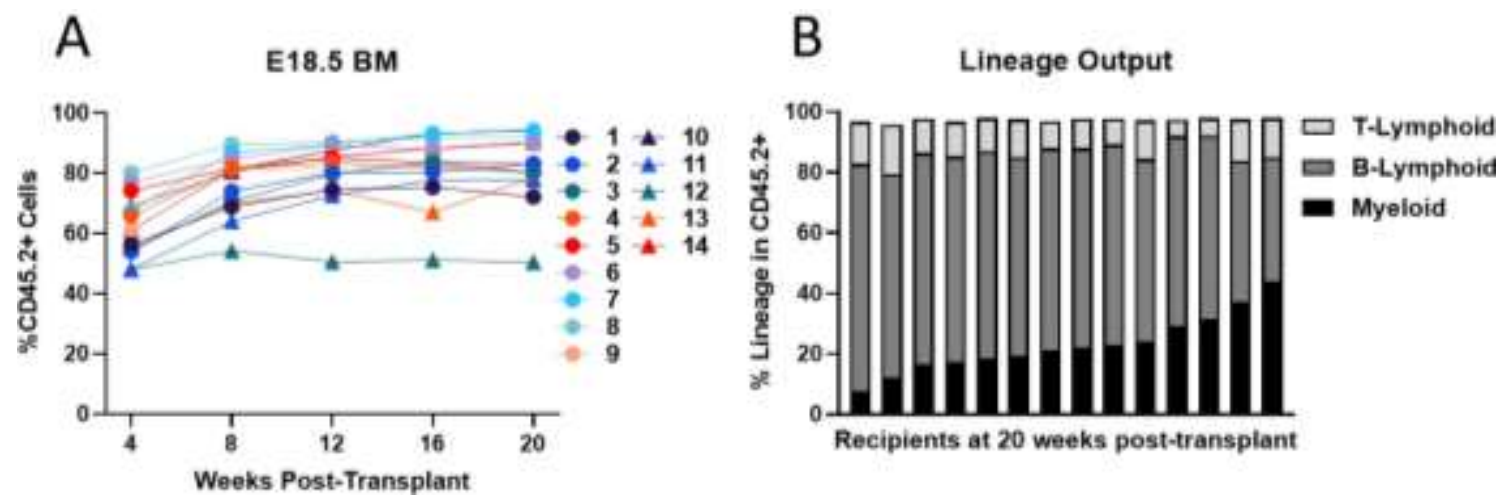

Figure 3-4. Primary transplantation of E18.5 FBM

A. Contribution of CD45.2 $2^{+}$donor E18.5 FBM to the PB of transplanted recipients over 20 weeks $(\mathrm{n}=14)$. B. T-lymphoid, B-lymphoid, and myeloid lineage output within the CD45.2 $2^{+}$compartment of PB in recipients at 20 weeks post-transplant $(n=14)$.
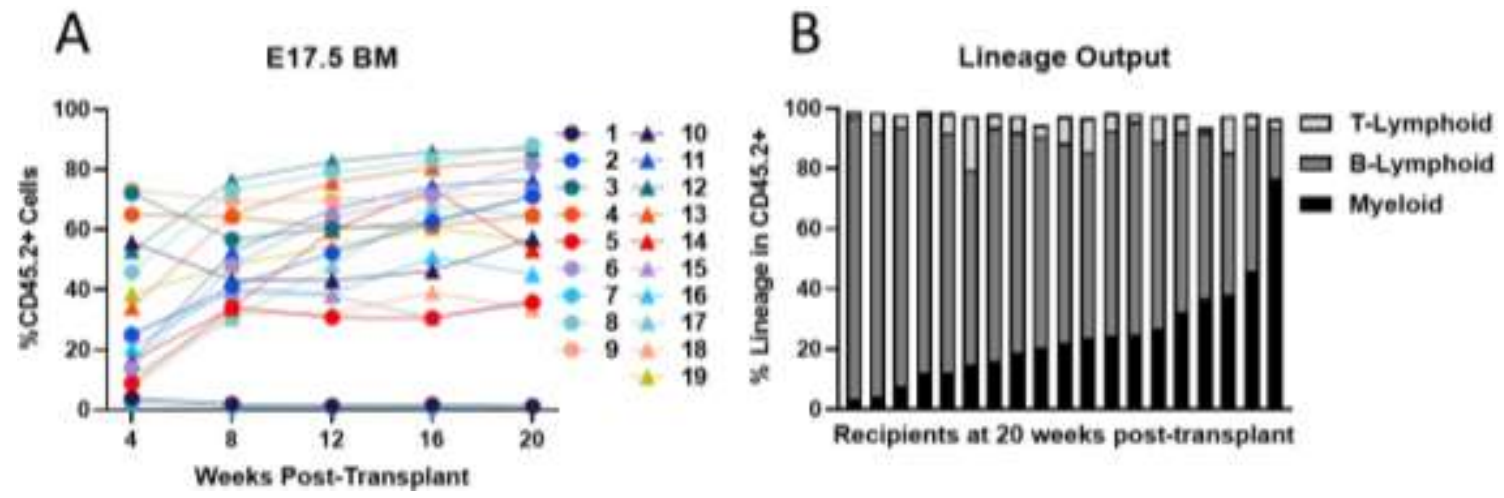

Figure 3-5. Primary transplantation of E17.5 FBM

A. Contribution of CD45.2 $2^{+}$donor E17.5 FBM to the PB of transplanted recipients over 20 weeks $(n=19)$. B. T-lymphoid, B-lymphoid, and myeloid lineage output within the CD 45.2 $2^{+}$compartment of PB in recipients at 20 weeks post-transplant $(n=19)$. 

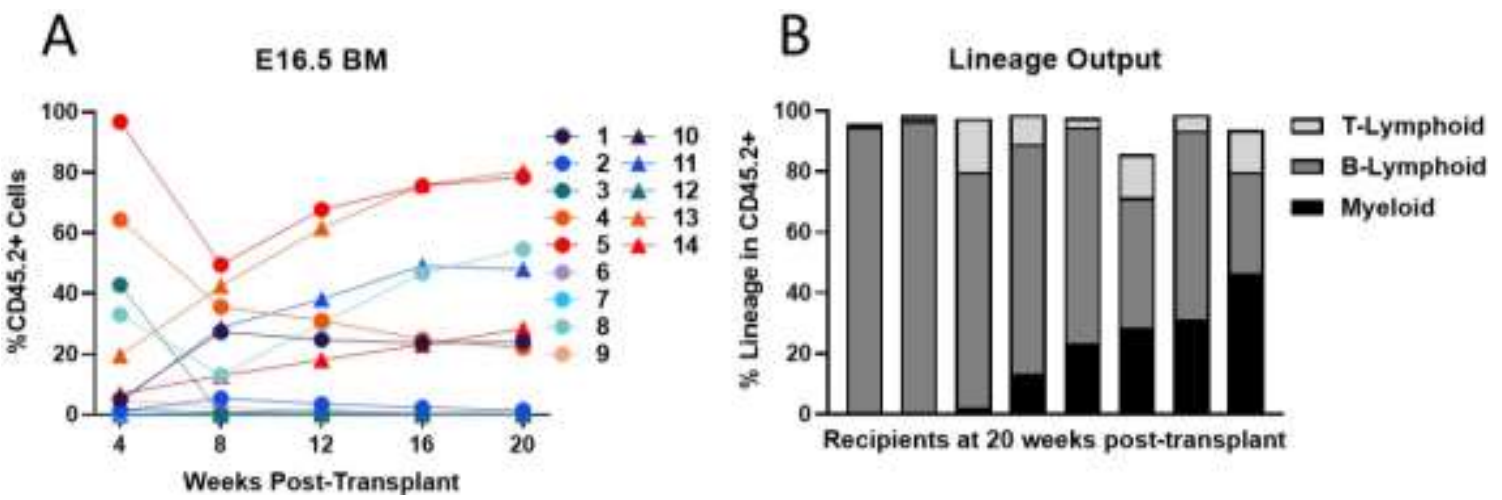

Figure 3-6. Primary transplantation of E16.5 FBM

A. Contribution of CD45.2 $2^{+}$donor E16.5 FBM to the PB of transplanted recipients over 20 weeks $(\mathrm{n}=14)$. B. T-lymphoid, B-lymphoid, and myeloid lineage output within the CD $45.2^{+}$compartment of PB in recipients at 20 weeks post-transplant $(n=8)$.
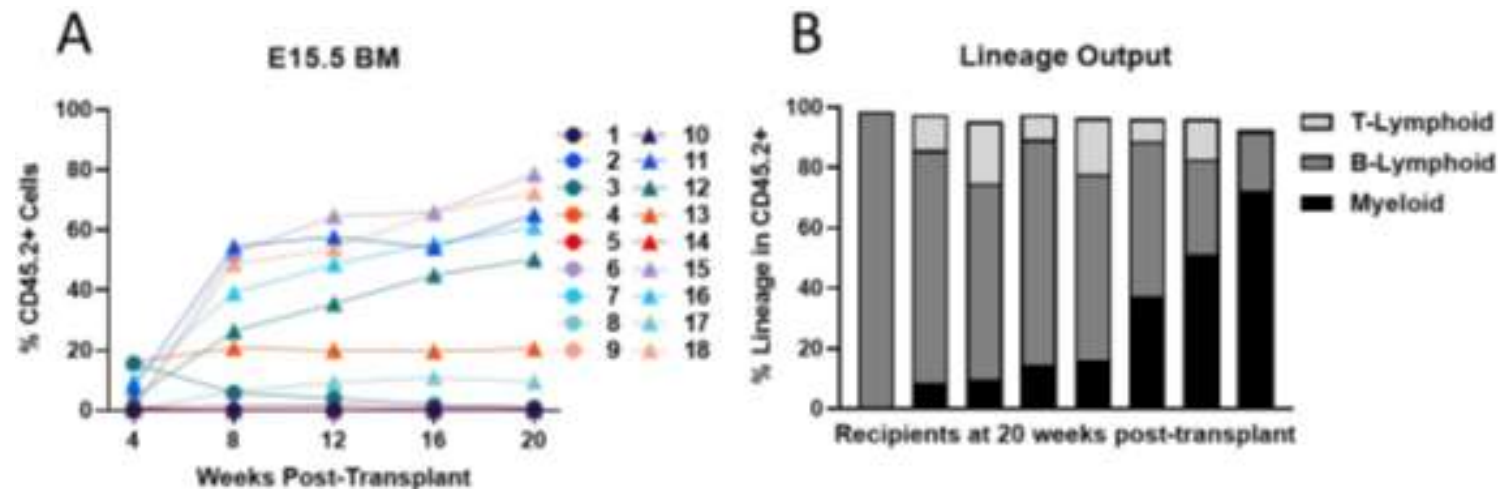

Figure 3-7. Primary transplantation of E15.5 FBM

A. Contribution of CD45.2 $2^{+}$donor E15.5 FBM to the PB of transplanted recipients over 20 weeks $(\mathrm{n}=18)$. B. T-lymphoid, B-lymphoid, and myeloid lineage output within the CD45.2 $2^{+}$compartment of PB in recipients at 20 weeks post-transplant $(n=8)$. 


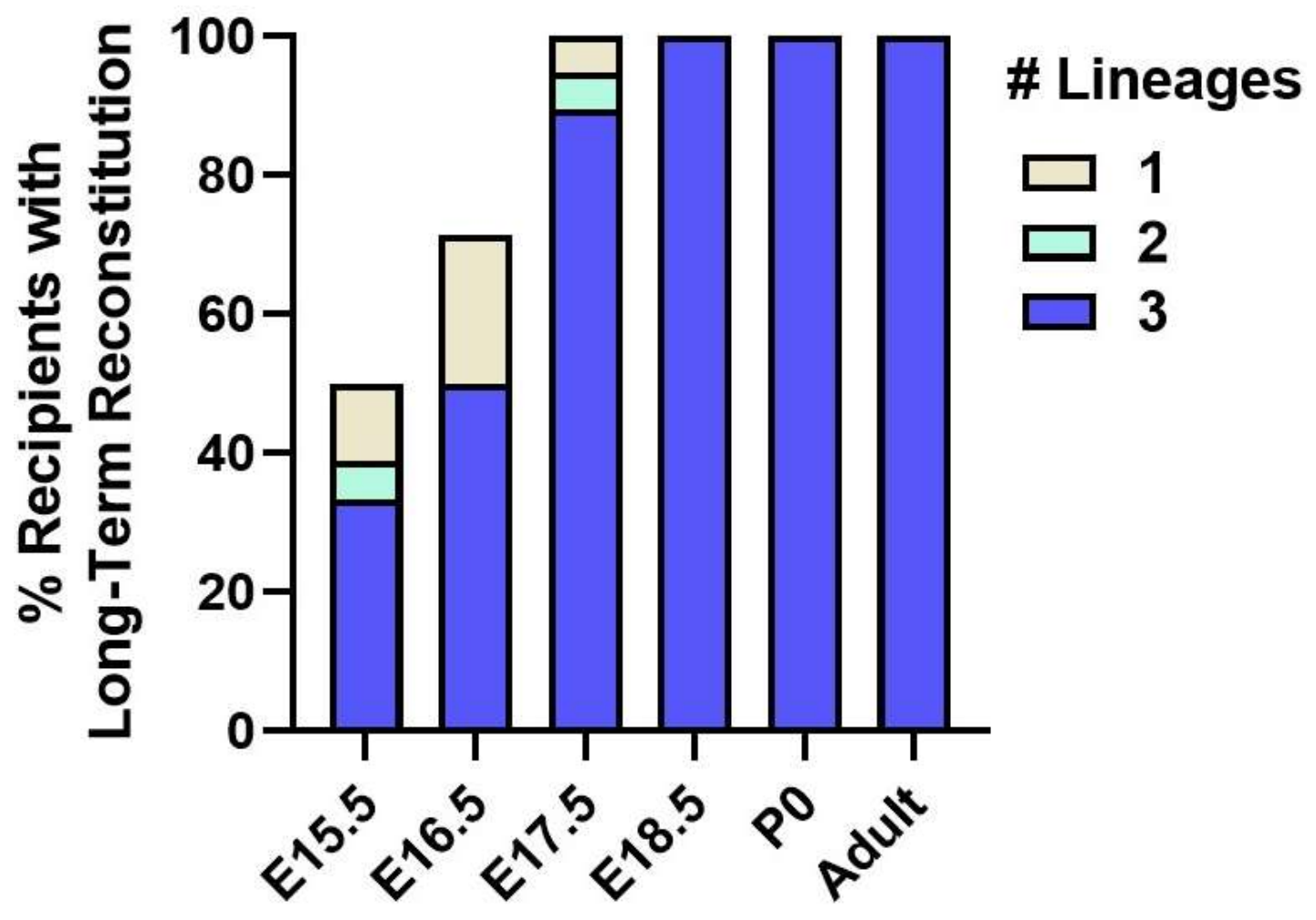

Figure 3-8. Primary transplant summary

Histogram summarizing the percentages of recipients receiving $1.5 \times 10^{6} \mathrm{BM}$ cells from E15.5-P0 and adult donors that displayed long-term reconstitution ( 20 weeks) from donor cells, including the number of lineages detected at the terminal (20 weeks post-transplant) bleed. 


\section{Bona Fide HSCs Are Present in the E15.5 FBM}

While we confirmed the presence of long-term repopulating cells in the E15.5 FBM, the gold standard for identification of bona fide HSCs is their ability to selfrenewal through secondary transplantation (Eaves 2015). Interestingly, the only attempt to secondarily transplant any FBM-derived hematopoietic tissue that we are aware of was only after a few weeks, and the recipients were only followed for $\sim 4$ weeks posttransplant (Bowie 2007). Therefore, while the presence of self-renewing bona fide HSCs in the FBM and early neonatal BM is presumed, we decided to secondarily transplant BM from E15.5, E16.5, E17.5, E18.5, P0, and adult primary recipients to confirm the presence of bona fide HSCs in these tissues. To do this, we isolated several aliquots of 5 x $10^{6}$ cells from single primary recipients and injected into lethally irradiated secondary recipients, followed by analysis of PB chimerism and lineage output (Figure 3-9). We also analyzed the contribution of donor cells to the BM HSPC compartments of primary recipients (Figure 3-9).

As expected, adult BM donor cells contributed robustly to all primary recipient BM HSPC compartments at levels similar to PB donor chimerism ( $\sim 80 \%)$ (Figure 3-10A), and secondary recipients displayed robust donor contribution to the PB (Figure 3-10B). In line with the lineage output of primary recipients, secondary recipients of adult BM displayed balanced lineage output in three independent transplants (Figure 3-10C). Similarly, P0 BM donor cells displayed robust contribution to all HSPC compartments in primary recipient BM (Figure 3-11A), and secondary recipients of P0 BM showed long-term reconstitution in three independent transplants (Figure 3-11B). The lineage output in secondary recipients of P0 BM was also balanced (Figure 3-11C). Together, these data show for the first time the presence of a substantial pool of bona fide HSCs in the P0 BM.

Analysis of the donor contribution to the HSPC compartments in primary recipients of E18.5 BM revealed that some recipients displayed reduced chimerism in the primitive HSPC compartments (LT-HSC, ST-HSC, MPPs) compared to the downstream committed HSPC compartment [CMP, GMP, MEP, common lymphoid progenitor (CLP)] (Figure 3-12A). This is a curious result, as one would expect that the donor contribution to downstream compartments would reflect the chimerism of upstream progenitors, and suggests that perhaps the progeny of E18.5 LT-HSCs, ST-HSCs, and MPPs have a competitive advantage over supportive cells within primary recipients. Nevertheless, secondary recipients of E18.5 FBM displayed long-term repopulation from donor cells in three independent transplants (Figure 3-12B), with one transplant displaying slightly higher levels of donor B-cell contribution than the other two (Figure 3-12C). These results provide the first evidence via long-term analysis of secondarily transplanted recipients of bona fide HSCs in the E18.5 FBM.

Similar to E17.5 PB donor chimerism, the E17.5 HSPC compartment donor chimerism of primary recipients displayed high stochasticity, suggesting the transplantation of fewer numbers of HSCs (Figure 3-13A). Interestingly, the trend of low chimerism in LT-HSCs compared to more committed progenitors found in recipients of 


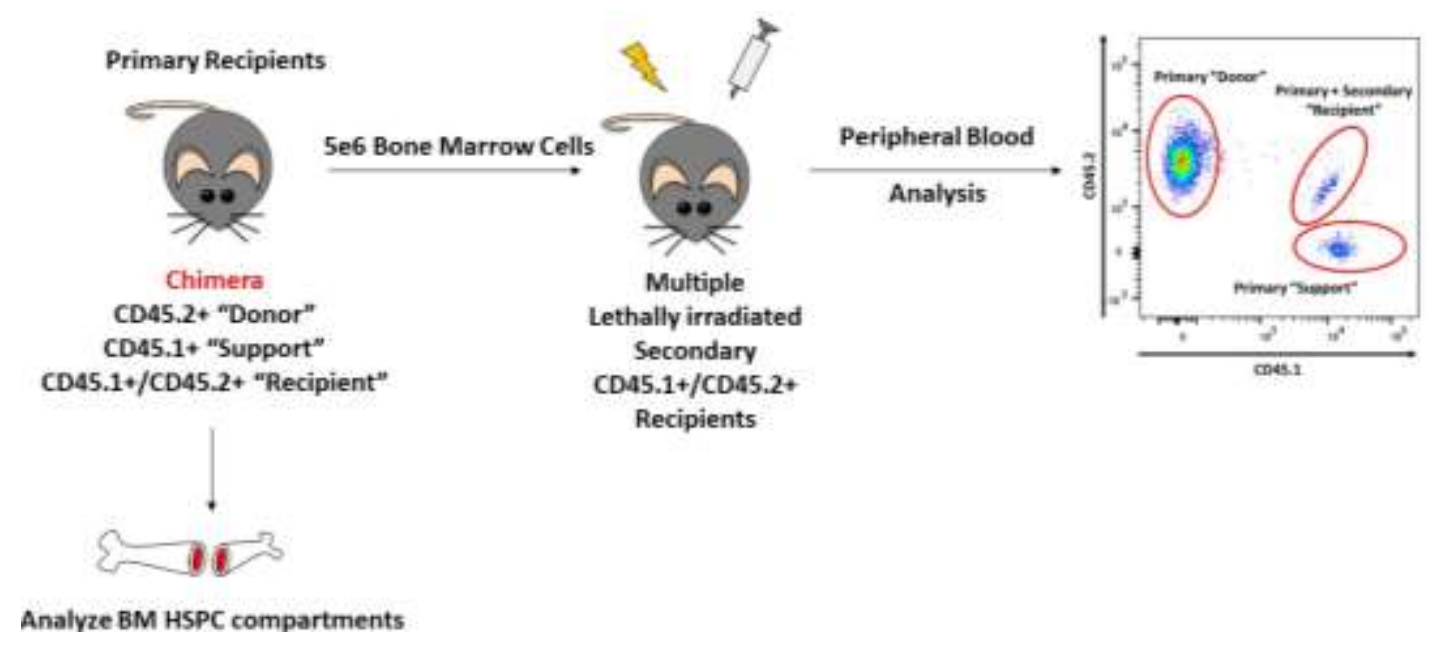

Figure 3-9. Secondary transplant of FBM

Schematic for secondary transplantation of fetal bone marrow (FBM) cells and BM HSPC compartment analysis of primary recipients. Five aliquots of $5 \times 10^{6}$ primary recipient BM cells were isolated from a single primary recipient and transplanted into five lethally irradiate CD $45.1^{+} / \mathrm{CD} 45.2^{+}$recipients. Simultaneously, the BM HSPC compartments of each primary recipient was analyze for donor cell contribution. Secondary recipient peripheral blood (PB) was analyzed for donor contribution over a period of 20 weeks. 


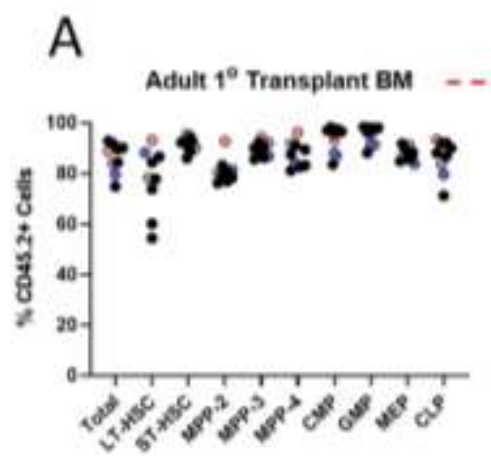

B
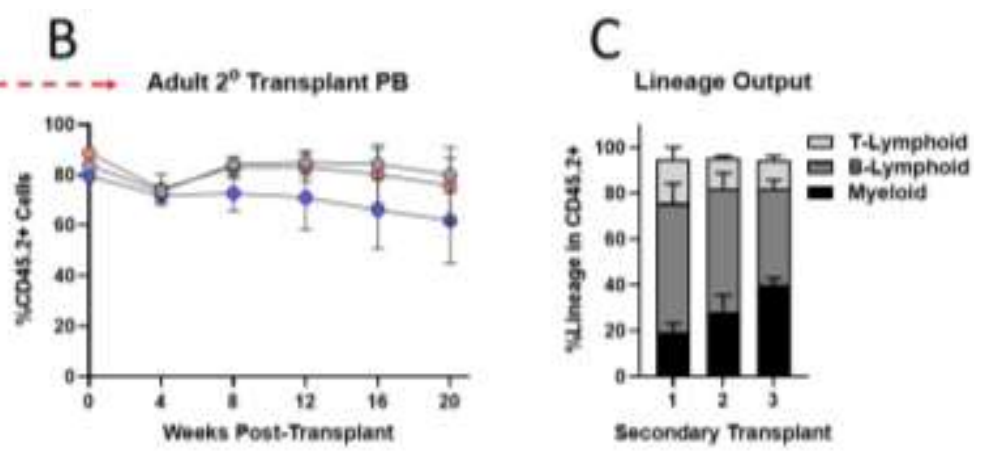

Figure 3-10. Secondary transplantation of adult BM

A. The CD45.2 $2^{+}$adult BM donor contribution to different HSPC compartments in primary recipients $(n=9)$. B. PB donor chimerism over 20 weeks in secondary recipients transplanted with aliquots of $5 \times 10^{6} \mathrm{BM}$ cells from the indicated color-coded primary recipients in A. Data represent mean and s.d. from 5 transplanted mice. C. T-lymphoid, B-lymphoid, and myeloid lineage donor cell output in secondary recipients at 20 weeks post-transplant. Data represent mean and s.d. from 5 transplanted mice.

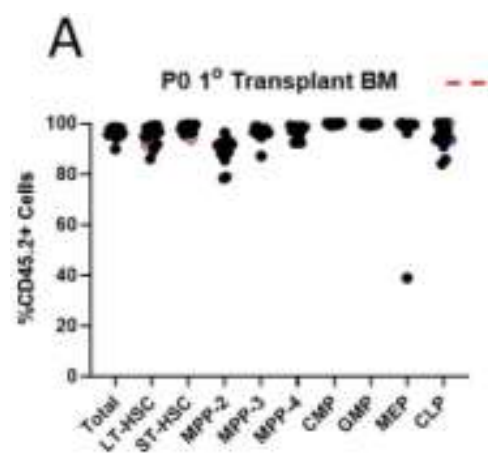

B

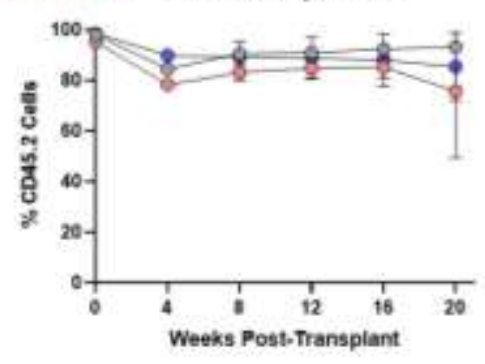

C Lineage Output

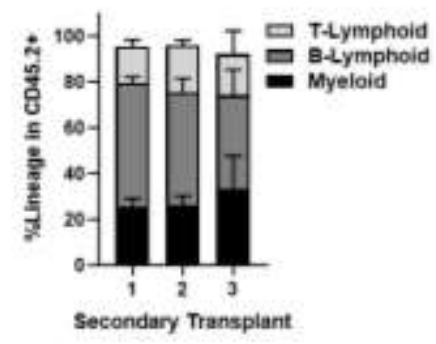

Figure 3-11. Secondary transplantation of P0 BM

A. The CD45.2 $2^{+}$P0 BM donor contribution to different HSPC compartments in primary recipients $(n=9)$. B. PB donor chimerism over 20 weeks in secondary recipients transplanted with aliquots of $5 \times 10^{6} \mathrm{BM}$ cells from the indicated color-coded primary recipients in A. Data represent mean and s.d. from 5 transplanted mice. C. T-lymphoid, B-lymphoid, and myeloid lineage donor cell output in secondary recipients at 20 weeks post-transplant. Data represent mean and s.d. from 5 transplanted mice. 


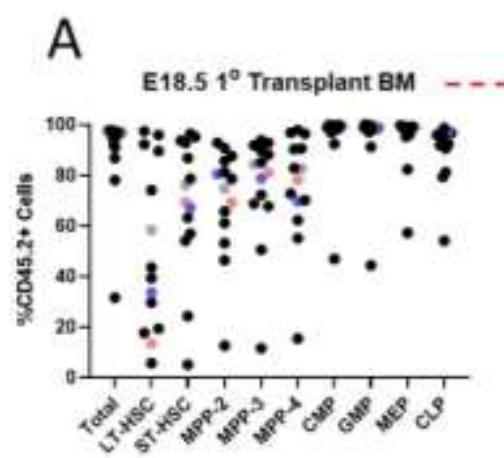

B
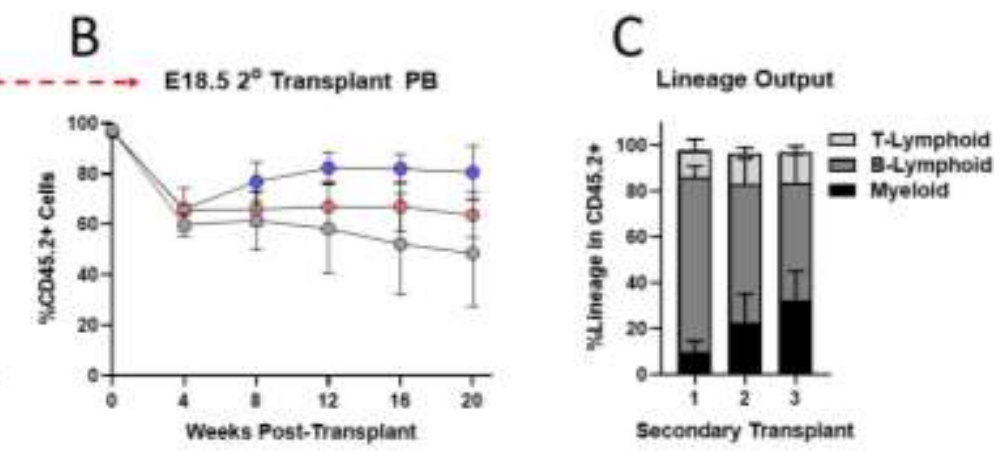

Figure 3-12. Secondary transplantation of E18.5 FBM

A. The CD45.2 $2^{+}$E18.5 FBM donor contribution to different HSPC compartments in primary recipients $(n=9)$. B. PB donor chimerism over 20 weeks in secondary recipients transplanted with aliquots of $5 \times 10^{6} \mathrm{BM}$ cells from the indicated color-coded primary recipients in A. Data represent mean and s.d. from 5 transplanted mice. C. T-lymphoid, B-lymphoid, and myeloid lineage donor cell output in secondary recipients at 20 weeks post-transplant. Data represent mean and s.d. from 5 transplanted mice.

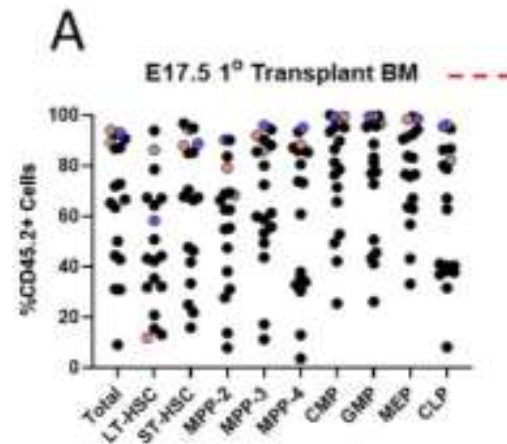

B
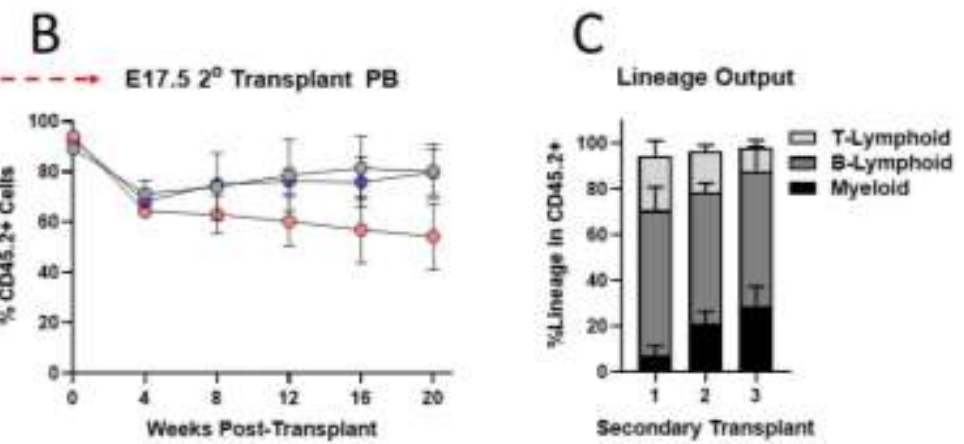

Figure 3-13. Secondary transplantation of E17.5 FBM

A. The CD45.2 $2^{+}$E17.5 FBM donor contribution to different HSPC compartments in primary recipients $(\mathrm{n}=9)$. B. PB donor chimerism over 20 weeks in secondary recipients transplanted with aliquots of $5 \times 10^{6} \mathrm{BM}$ cells from the indicated color-coded primary recipients in A. Data represent mean and s.d. from 5 transplanted mice. C. T-lymphoid, B-lymphoid, and myeloid lineage donor cell output in secondary recipients at 20 weeks post-transplant. Data represent mean and s.d. from 5 transplanted mice. 
E18.5 FBM was seen in recipients of E17.5 FBM, although this difference was not as pronounced (Figure 3-13A). Secondary recipients displayed long-term PB repopulation from E17.5 donor cells (Figure 3-13B), and all transplants showed tri-lineage output from donor cells at 20 weeks post-transplantation, although one transplant did show higher donor B-lymphoid lineage output (Figure 3-13C). This is the first confirmed existence of bona fide HSCs in E17.5 FBM, although it appears that there are fewer HSCs at this timepoint compared to later timepoints.

Considering the reduced long-term repopulating potential in primary recipients of E16.5 FBM as confirmed by this study and others (Coşkun 2014), it was critical to determine if the E16.5 FBM was a source of bona fide HSCs. Upon examination of the donor contribution to primary recipient HSPC BM populations, we found that most mice showed low levels of chimerism in all compartments, but there was a trend for greater donor chimerism in downstream progenitors (Figure 3-14A). Interestingly, upon secondary transplant of E18.5 FBM, one of the secondary transplants contained only $1.26 \%$ donor contribution at 20 weeks (Figure 3-14B). However, upon inspection of the primary transplant BM HSPC compartment donor contribution, the recipients of this secondary transplant were injected with BM that contained a LT-HSC compartment composed of only $1.57 \%$ donor cells (Figure 3-14A). Indeed, other secondary recipients showed PB chimerism levels similar to the LT-HSC donor compartment of primary recipients (Figure 3-14A-B). While all secondary transplants displayed tri-lineage output from donor cells, two transplants showed reduced myeloid output at the expense of lymphoid output, and in the case of one transplant this was similar to the lineage distribution in the primary recipient (Figure 3-14C). These data provide the first evidence of bona fide HSCs in the E16.5 FBM, although as the primary transplant data suggests (Figure 3-6), transplantable HSCs are rare at this timepoint.

We next examined the HSPC BM donor contribution in recipients transplanted with E15.5 FBM. As expected, the majority of recipients showed little to no reconstitution in the progenitor compartments (Figure 3-15A). However, in the recipients that did show reconstitution there was again a trend for more committed progenitors to show higher levels of donor chimerism than LT-HSCs (Figure 3-15A). Three independent secondary transplantations confirmed that E15.5 FBM donor cells could contribute to long-term repopulation, suggesting the presence of bona fide HSCs in the $\mathrm{BM}$ as early as E15.5 (Figure 3-15B). Interestingly, one secondary transplant showed $81 \%$ donor B-lymphoid lineage output and $<1 \%$ myeloid lineage output (Figure 3-15C). The secondary recipients constituting this transplant were injected with BM from a primary recipient that also displayed a B-lymphoid biased lineage output, suggesting the presence of bona fide lineage-biased HSCs in the E15.5 FBM. Therefore, the heterogeneity associated with the HSC pool in the adult BM appears to be present as early as E15.5.

These secondary transplants provide the first confirmation of the existence of rare, bona fide HSCs in the BM as early as E15.5. Analysis of the BM HSPC compartments of primary recipients suggest that the progeny of HSCs from all FBM timepoints (E15.5E18.5) may have a competitive advantage post-transplantation, as their donor chimerism 


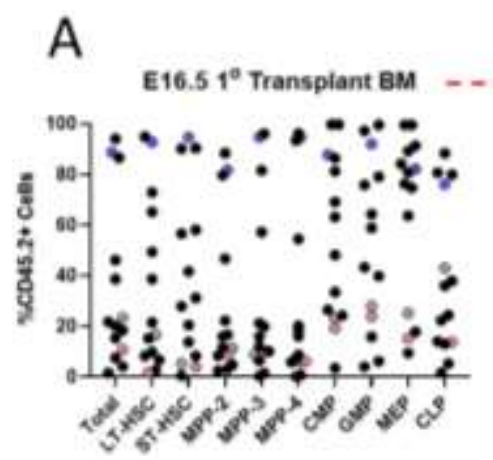

\section{B}
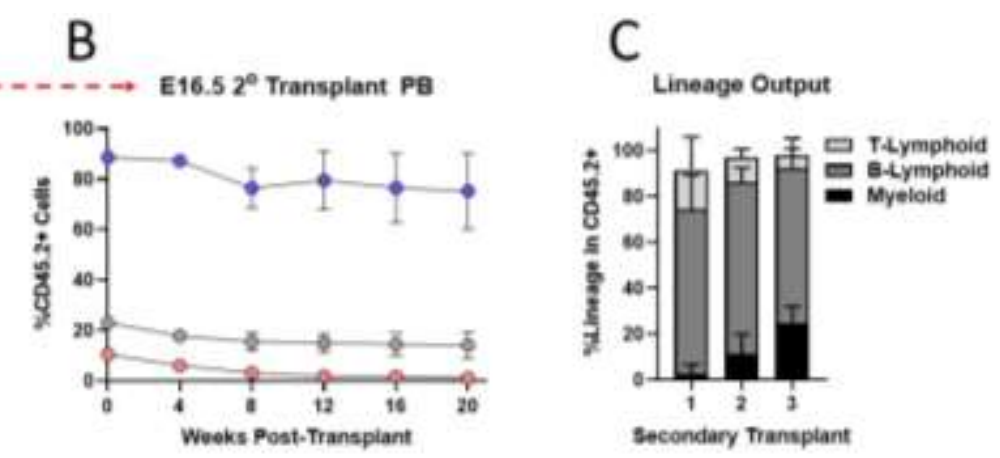

Figure 3-14. Secondary transplantation of E16.5 FBM

A. The CD45.2 $2^{+}$E16.5 FBM donor contribution to different HSPC compartments in primary recipients $(n=9)$. B. PB donor chimerism over 20 weeks in secondary recipients transplanted with aliquots of $5 \times 10^{6} \mathrm{BM}$ cells from the indicated color-coded primary recipients in A. Data represent mean and s.d. from 5 transplanted mice. C. T-lymphoid, B-lymphoid, and myeloid lineage donor cell output in secondary recipients at 20 weeks post-transplant. Data represent mean and s.d. from 5 transplanted mice.
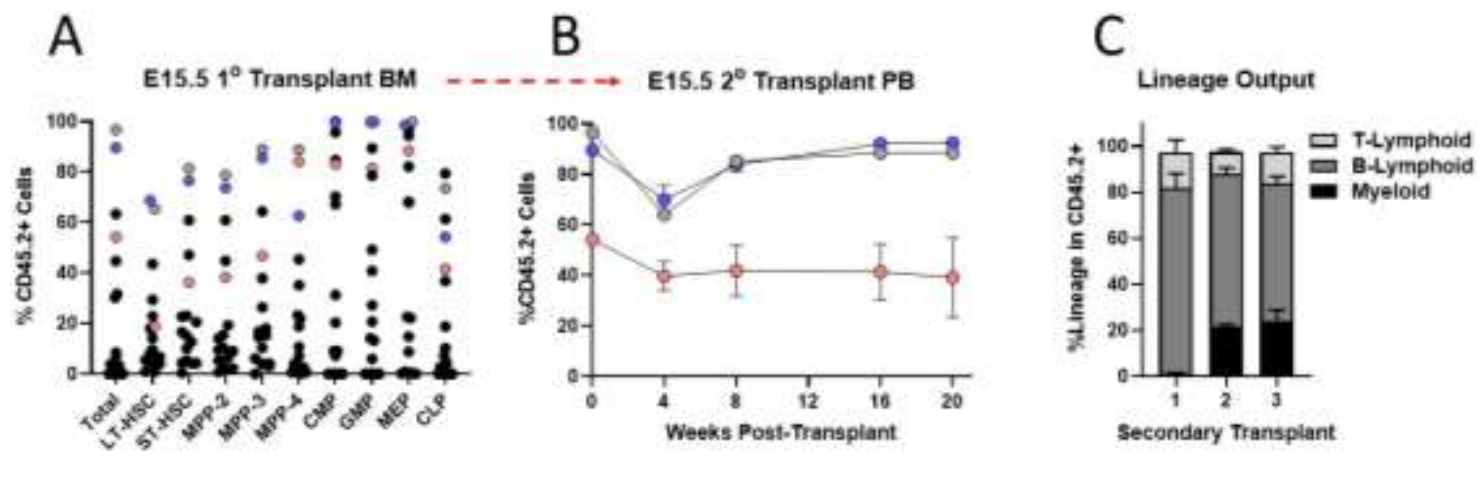

Figure 3-15. Secondary transplantation of E15.5 FBM

A. The CD45.2 ${ }^{+}$E15.5 FBM donor contribution to different HSPC compartments in primary recipients $(n=9)$. B. PB donor chimerism over 20 weeks in secondary recipients transplanted with aliquots of $5 \times 10^{6} \mathrm{BM}$ cells from the indicated color-coded primary recipients in A. Data represent mean and s.d. from 5 transplanted mice. C. T-lymphoid, B-lymphoid, and myeloid lineage donor cell output in secondary recipients at 20 weeks post-transplant. Data represent mean and s.d. from 5 transplanted mice. 
exceeds the chimerism from upstream compartments (Figures 3-12, 3-13, 3-14, and 3-15). This trend is not seen in primary recipients of P0 BM (Figure 3-11), suggesting the presence of a shift in the functional characteristics of progenitors around birth. Finally, heterogeneity in the BM HSC pool can be detected as early as E15.5 (Figure 315C), suggesting that HSCs either acquire this heterogeneity upon entry into the FBM or prior to FBM colonization.

\section{HSCs Can Be Found in All Sources of FBM at E15.5}

While we have demonstrated the presence of bona fide HSCs in the E15.5 FBM, others have only detected long-term repopulating cells in the FBM as early as E16.5 (Coşkun 2014). However, we assayed the entire skeleton except the skull while other groups only assayed long bones (Coşkun 2014). This suggests that HSCs may be present in other sources of FBM before the establishment of HSCs in the long bones at E16.5. To test for the presence of HSCs in multiple bone sources at E15.5, we isolated BM from three bone sources: 1.) the forelimbs, including the scapula, humerus, radius, and ulna, 2.) the hindlimbs, including the pelvis, femur, tibia, and fibula, and 3.) the trunk, including the spine, ribcage, and sternum. Each BM source was isolated from individual E15.5 embryos, and the entire BM suspension was injected into a lethally irradiated recipient (Figure 3-16). In this way, the contribution from each BM source within an individual embryo could be tracked for the presence of long-term repopulating cells.

Only 2/12 recipients transplanted with E15.5 forelimbs showed long-term repopulation $>1 \%$, and the donor contribution in one of the recipients only reached $1.7 \%$ (Figure 3-17A). Therefore, while the forelimbs can contain HSCs at E15.5, these HSCs appear to be rare and likely in low frequencies when present. Meanwhile, 5/14 recipients transplanted with E15.5 hindlimbs displayed long-term repopulation $>1 \%$, and two of these recipients reached a donor chimerism $>50 \%$, suggesting that HSCs are more frequent and in greater numbers within the E15.5 hindlimbs compared to the forelimbs (Figure 3-17B). Finally, 6/14 recipients transplanted with E15.5 trunks - which contain the highest BM cellularity within the entire E15.5 skeleton-were reconstituted with donor cells at levels $>1 \% 20$ weeks post-transplant, and 4/6 of these recipients displayed donor chimerism $>40 \%$ (Figure 3-17C). This suggests that the BM source containing the most HSCs at E15.5 is likely to be the trunk, although given that the trunk has greater cellularity than the forelimbs or hindlimbs, it is still possible that the other sources of BM have greater frequencies of HSCs.

We were also able to track the contribution of different BM sources within an E15.5 fetus towards long-term repopulation of recipients, allowing us to determine if HSCs are present in multiple sites in the FBM at the same time (Figure 3-17A). Interestingly, 2/11 donors contained transient repopulating cells in the hindlimbs alone followed by long-term repopulating cells in the forelimb and trunk, while no donors contained long-term repopulating cells that were only contained within the hindlimbs (Figure 3-17D). In contrast, one donor contained transient and long-term repopulating cells specific to the forelimbs while another donor contained trunk-specific transient (arising at 8 weeks post-transplant) and long-term repopulating cells (Figure 3-17D). 

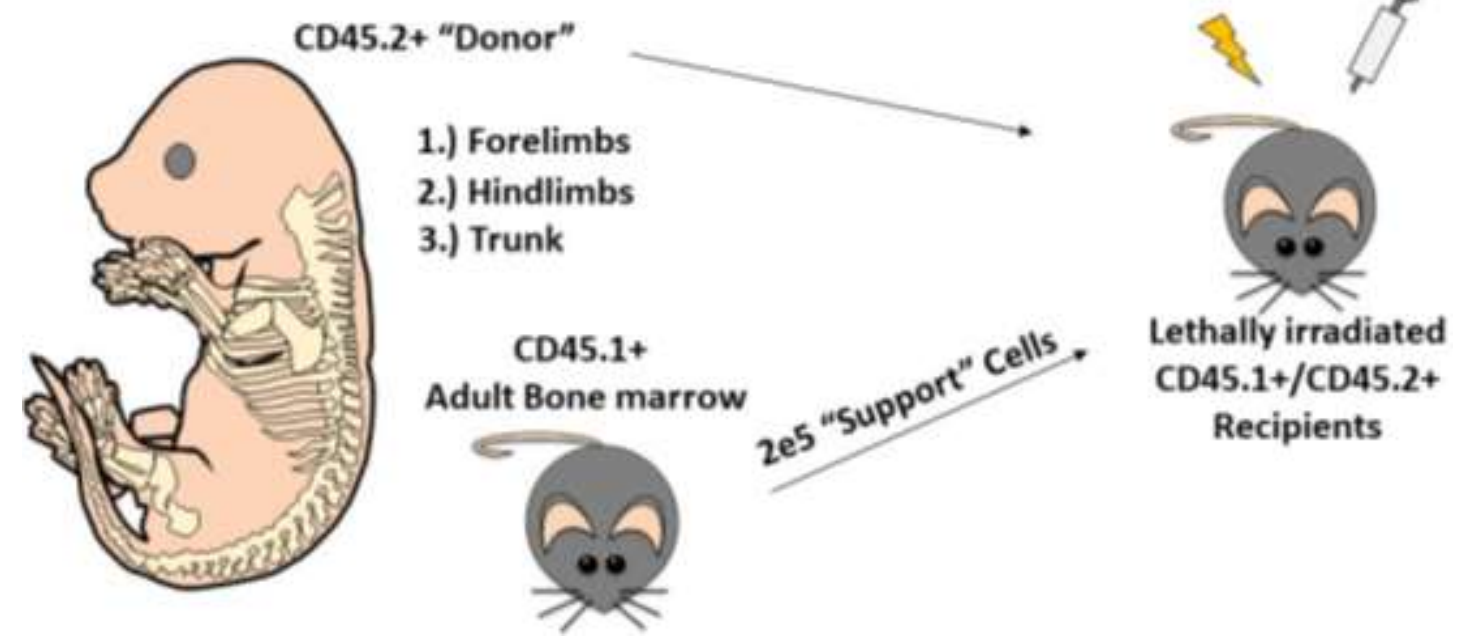

Figure 3-16. Transplantation of individual E15.5 fetal bones

Schematic depicting primary transplantation of BM cells from individual fetal bone sources in E15.5 embryos. The forelimbs, hindlimbs, and trunk bones were dissected from $\mathrm{CD} 45.2^{+}$donor embryos, processed to create single-cell suspensions, and injected separately into lethally irradiated recipients along with $2 \times 10^{5} \mathrm{CD} 45.1^{+}$supportive cells. 

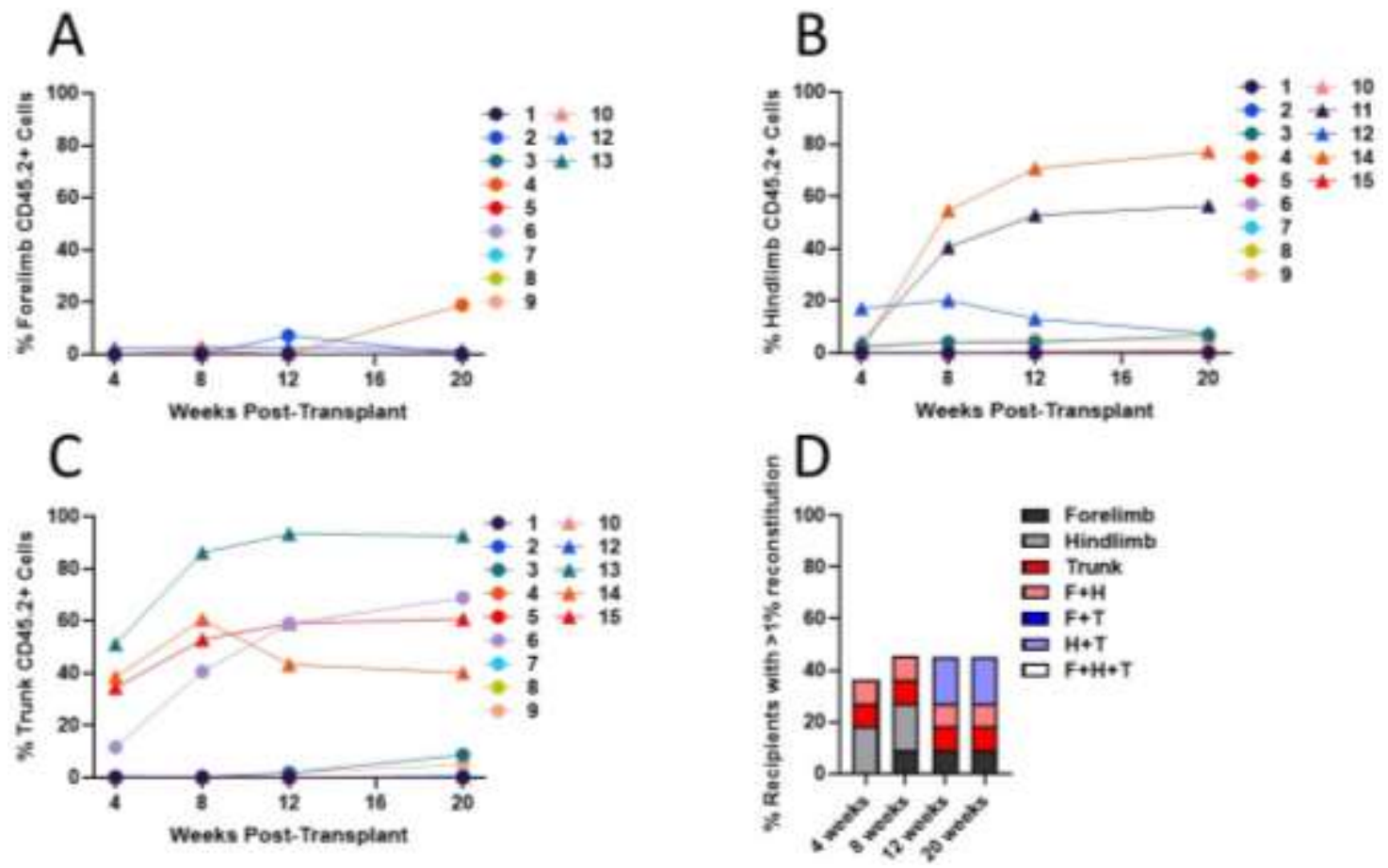

Figure 3-17. HSCs are found in all E15.5 BM sources

A. The CD45. $2^{+}$donor cell contribution to the PB of irradiated recipients injected with E15.5 forelimb FBM cells $(n=12)$. B. The CD45.2 $2^{+}$donor cell contribution to the PB of irradiated recipients injected with E15.5 hindlimb FBM cells $(n=14)$. C. The CD45.2 ${ }^{+}$ donor cell contribution to the PB of irradiated recipients injected with E15.5 trunk FBM cells $(n=14)$. D. Histogram depicting the percentage of recipients with $>1 \%$ donor cell contribution to the $\mathrm{PB}$ at $4,8,12$, and 20 weeks post-transplant from different bone sources within the same E15.5 embryo $(n=11)$. For A-C, the recipient numbers correlate across BM tissues, representing tissues coming from individual E15.5 embryos. 
A final donor displayed transient and long-term repopulating cells contained within the forelimb and hindlimb BM, while 6/11 donors displayed no reconstitution from any bone source (Figure 3-17D). It is difficult to make conclusions about the dynamics of HSC arrival in the different sources of BM within the E15.5 fetus given that only the recipients from 11 donors (33 total recipients) survived 20 weeks post-transplant, and only recipients from 5/11 donors showed any level of reconstitution (Figure 3-17D). However, given that no long-term reconstitution is present in hindlimbs alone, but forelimbs and trunks display long-term reconstitution either alone or in combination with hindlimbs, it is possible that the forelimbs and trunk may be preferentially colonized by HSCs followed by the colonization of hindlimbs.

Together these data unequivocally point to the presence of HSCs at E15.5 in all sources of BM, suggesting that while our transplantation of the entire skeleton is a more robust method for identifying the HSC potential in early FBM, it should not preclude the identification of HSCs in the long bones of E15.5 embryos.

\section{The FBM HSPC Compartment Is Compositionally Distinet From FL and Adult BM}

After confirming the existence of bona fide HSCs in the E15.5 FBM, we next wanted to thoroughly characterize the immunophenotypic HSPC compartment from the initial seeding of HSPCs at E15.5 to the proposed developmental shift in HSCs around 4 weeks post-birth (P28) from an actively cycling to a primarily quiescent phenotype (Bowie 2006, Bowie 2007). Importantly, we also wanted to determine if the HSPC compartment from the BM was similar in composition to the liver at the same developmental timepoints. To do this, we isolated the liver and the BM from entire skeletons of mice aged E15.5 to P28 and used flow cytometry to assess the frequencies of several immunophenotypic progenitors within the LSK compartment, including LTHSCs, ST-HSCs, MPP2s, MPP3s, and MPP4s (Pietras 2015) (Figure 3-18).

Upon inspection of liver HSPCs, we found that HSPC frequencies in the fetal and early neonatal liver remained relatively constant with MPP4s being the dominant phenotype until around P6, at which point there was a slight shift to a more MPP3dominant phenotype (Figure 3-19A). However, there was a remarkable difference in the HSPC frequencies within the BM at similar timepoints, as the E15.5-E18.5 HPSC compartment was dominated by immunophenotypic MPP2 cells (Figure 3-19B). Indeed, the MPP-2 and MPP-4 frequencies in the E15.5 FBM were 0.64 and 0.09 respectively, and these values remained relatively unchanged until E19 (Figure 3-19B). Meanwhile, the MPP-2 and MPP-4 frequencies in the E15.5 FL were 0.09 and 0.38 respectively, remaining relatively constant until P6 (Figure 3-19A). Interestingly, while immunophenotypic LT-HSCs could be identified in the early FBM, the frequency of this population within the HSPC was $\sim 0.01$ from E15.5-E17.5 until jumping to 0.03 at E18.5, a frequency similar to that seen in the P28 BM (Figure 3-19B).

By counting the number of cells within tissues per individual during our analyses, we were able to calculate the projected total number of immunophenotypic progenitors 


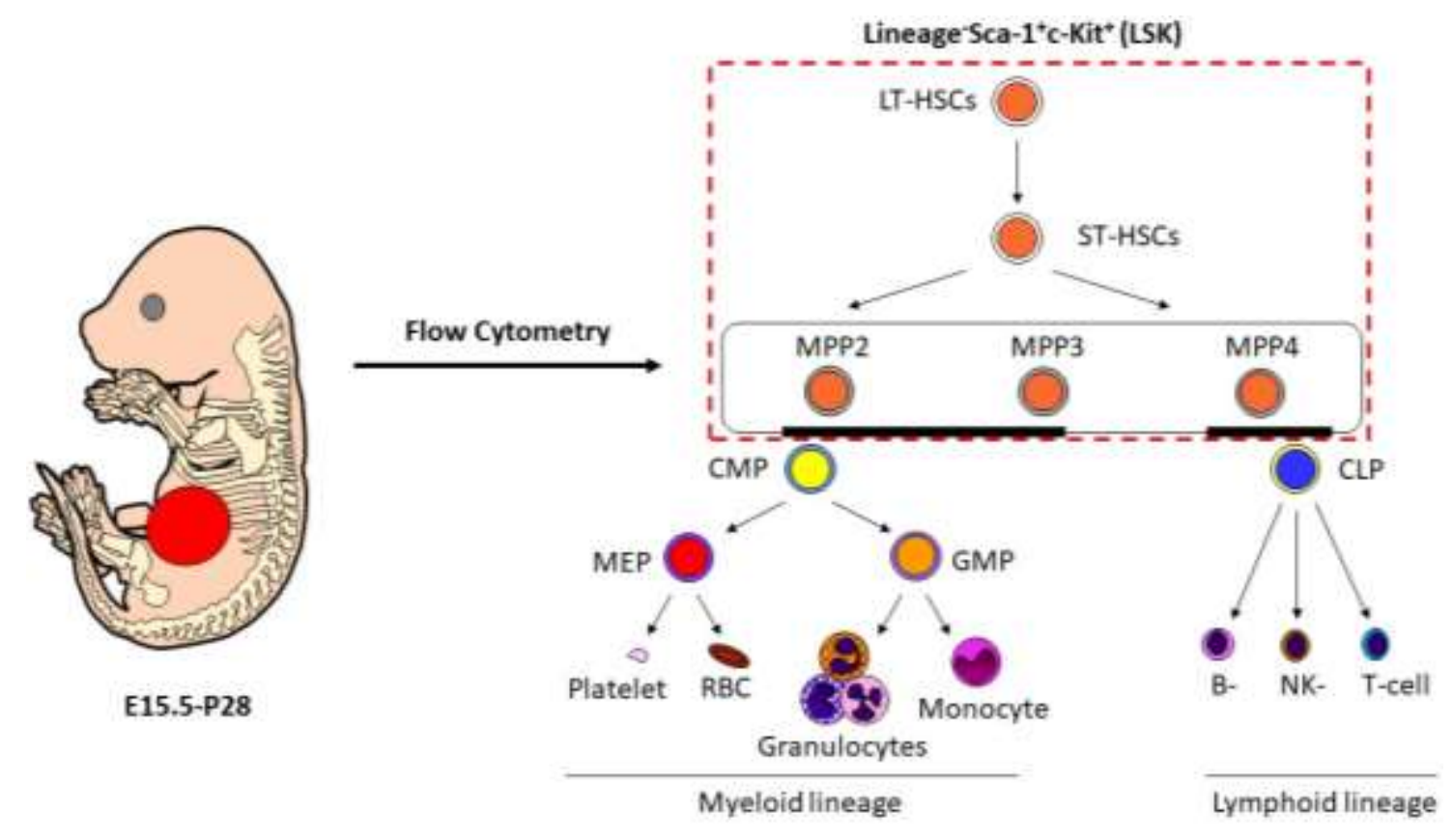

Figure 3-18. Analysis of HSPCs across BM and liver ontogeny

Schematic depicting the method for analyzing the HSPC compartments of murine BM and liver across development. Bones and livers were dissected from E15.5-P28 mice, stained with the appropriate antibodies, and analyzed for the presence of several progenitor cells (as defined by Pietras 2015) within the LSK compartment (LT-HSCs, ST-HSCs, MPP2s, MPP3s, and MPP4s). 

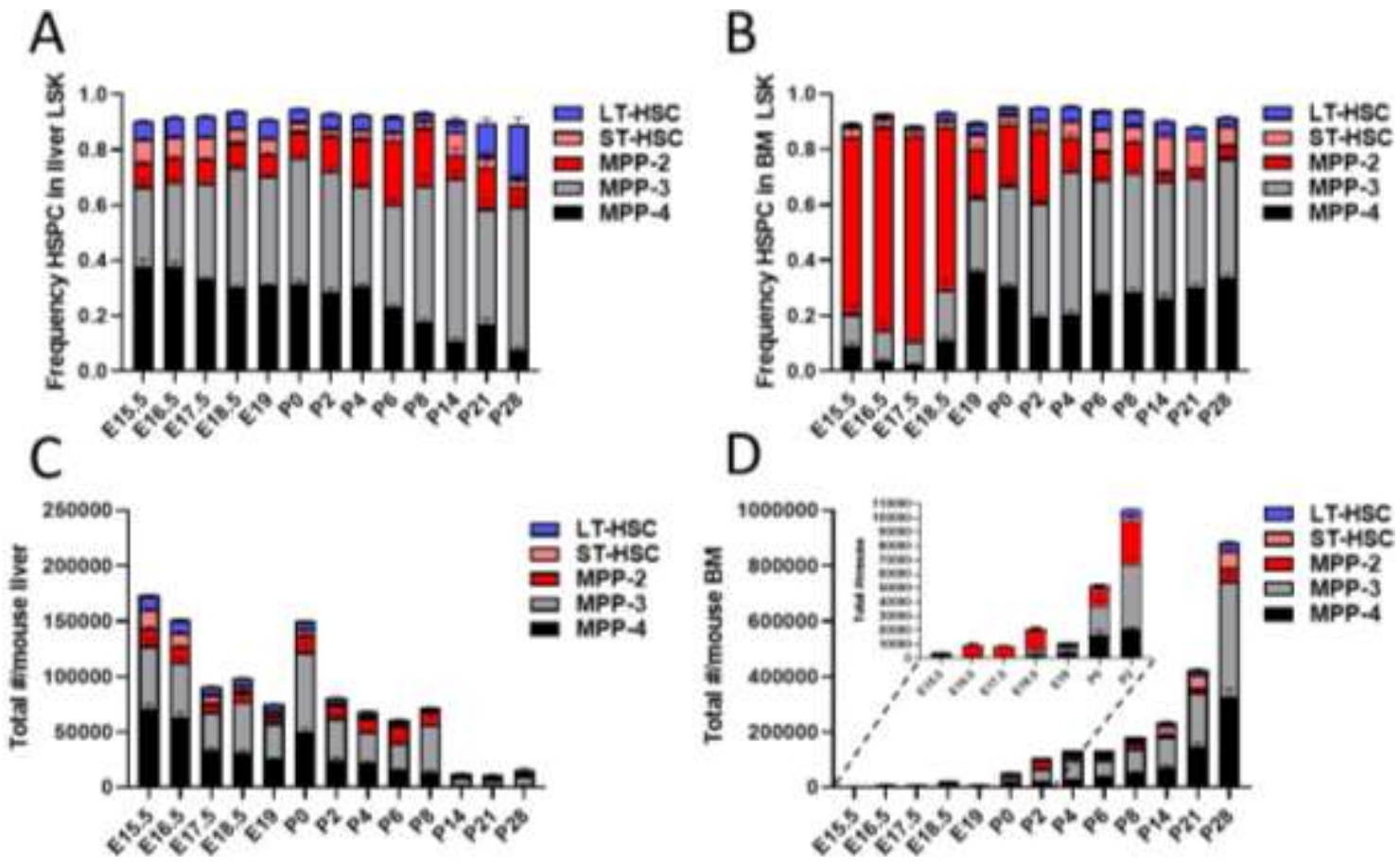

Figure 3-19. Frequency and total number of HSPCs in BM and liver

A-B. The frequency of different HSPC populations within the liver LSK compartment (A) and BM LSK compartment (B) from late gestation to adulthood in C57B6/J mice (n $=10-17$ from 2-3 independent litters) . C-D. The calculated absolute number of immunophenotypic HSPCs in the liver (C) or BM (D) from late gestation to adulthood in C57B6/J mice ( $\mathrm{n}=10-17$ from 2-3 independent litters). All data represent means with SE. 
within the liver or BM of an individual mouse at each timepoint. It is thought that after expanding in the FL, HSPCs migrate to the FBM from around E15.5 until after birth (Medvinsky 2011, Khan 2016). Therefore, we expected to see a decrease in the total number of HSPCs in the FL and a concomitant increase in FBM HSPC numbers starting at E15.5. Three trends characterized the dynamics of HSPC numbers in the liver. First, there was a considerable decrease in the total numbers of all progenitors from E15.5 until right before birth, at which point there was a burst in the total numbers of all progenitors, especially in the MPP2 and MPP3 compartments (Figure 3-19C). Second, following the burst of progenitor numbers at $\mathrm{P} 0$, there was a precipitous drop in the total number of progenitors at P2, which remained relatively constant until P8 (Figure 3-19C). Lastly, HSPCs numbers dropped dramatically from P8 to P14, from $\sim 60,000$ to $\sim 11,000$ total HSPCs (Figure 3-19C). Together, these data suggest that aside from an uncharacteristic jump in HSPC numbers in the FL right after birth, liver HSPCs are largely disappearing from E15.5 to P8 and are essentially exhausted at by P14 compared to earlier timepoints.

Tracking the total numbers of HSPC in the BM revealed the opposite trend. While the P28 BM contained $\sim 885,000$ total immunophenotypic HSPCs $-84 \%$ of which were either MPP3s or MPP4s - the E15.5 FBM contained only 4,139 immunophenotypic HSPCs, and this number steadily increased from E15.5 to P28 with only a small dip in total numbers at E19 (Figure 3-19D). Indeed, the number of immunophenotypic HSCs increases from only 64 at E15.5 to $\sim 5,000$ at P2 (Figure 3-19D). Closer inspection of the E15.5 to P2 total HSPCs numbers also revealed that the shift in HSPC frequencies from an MPP2-dominant phenotype to an "adult-like" (P28) phenotype around birth appears to be mainly due to an increase in the total numbers of other HSPCs, not a decrease in the number of MPP2s. For instance, total numbers of MPP2s increased by 10 -fold from E16.5 to P2, while LT-HSCs, ST-HSCs, MPP3s, and MPP4s increased by factors of 35, 13,45 , and 58 , respectively (Figure 3-19D).

Together, these data show that the HSPC compartments of the FL and FBM are distinct, even at similar developmental timepoints. Also, while the composition of the HSPC compartment in the FL does not undergo dramatic change until around P6-a similar timepoint at which HSPCs appear to be lost from the liver- the same compartment in the FBM drastically changes right around birth from an MPP2-dominant to an MPP3/MPP4-dominant phenotype, similar to the HSPC distribution in adult BM. This shift appears to be due to an increase in the absolute number of HSPCs, not a decrease in the numbers of MPP2 cells.

\section{All FL HSPCs Have the Potential to Migrate to the FBM}

The presence of an MPP2-dominant HSPC compartment in the E15.5 FBM was intriguing, as we assumed that LT-HSCs would be the dominant HSPC subpopulation at the earliest FBM timepoints followed by expansion of downstream progenitors. We therefore decided to test whether all HSPC subsets had the ability to migrate from the FL to the FBM during late gestation. Migration from the FL to the FBM is dependent on CXCL12/CXCR4 chemoattraction, as $C X C L 12^{-/-}$mice display almost no repopulating 
ability from the E18.5 BM, and the HSCs in these animals appear to be trapped in the PB (Ara 2003). This is thought to be due to the lack of Cxcl12 expression from FBM niche cells, as the cell surface expression of CXCR4 in HSCs is thought to be a key component to their retention in $\mathrm{Cxcl1} 2^{+}$adult BM niches (Crane 2017).

We first isolated HSPCs from E15.5-P0 livers and analyzed each compartment for the expression of cell surface CXCR4 to determine if they have the machinery necessary to migrate towards a CXCL-12 gradient (Figure 3-20). We found that all HSPCs from E15.5-P0 liver contained populations of $\mathrm{CXCR}^{+}$cells, suggesting that these cells would have the ability to respond to CXCL-12 (Figure 3-20). We next performed a series of ex vivo transwell migration assays to determine if CXCR4 ${ }^{+} \mathrm{HSPCs}$ in the E15.5-P0 liver could migrate towards CXCL-12 (Figure 3-21). In this assay, we isolated HSPCs from FL at gestational and P0 timepoints and dispensed the cells in transwell plates. The top portion of the well contained the cells of interest and serum/cytokine-free media, while the bottom portion of the well contained media with or without soluble CXCL-12 (Figure 3-21). After a 5-hour incubation to allow for migration of cells towards CXCL12 , the bottom portion of the well was collected and plated in methylcellulose to determine if any colony forming progenitors migrated in response to CXCL-12 (Figure 3-21).

As expected, no migration was observed towards wells lacking CXCL-12 (Figure 3-22A). However, all liver HSPCs from E15.5-P0 showed migratory potential towards CXCL-12 (Figure 3-22B). This shows that all HSPCs from the FL have the potential to migrate toward the FBM niche at the earliest timepoints, and suggests a model in which the FBM is populated by a mixture of migratory HSPCs as well as HSPCs potentially arising from resident LT- and ST-HSCs.

\section{FBM MPP2s Are Functionally Distinct From FL and Adult MPP2s}

Upon closer inspection of the frequency of HSPCs in the FBM around birth, we noticed that the frequency of CD $45^{+}$cells in the BM sharply increases around E18.5 and are sustained through P2, but the frequency of HSPCs within the CD45 ${ }^{+}$compartment displays bimodal fluctuation, as $\mathrm{CD}^{+} 5^{+}$HSPCs peak at E15.5, sharply decrease around E18.5, and then sharply increase again at the newborn stage (Figure 3-23). This suggested to us that at early (E15.5-E18.5) timepoints, HSPCs are not contributing as robustly to blood production in the FBM. However, by E18.5 and beyond, HSPCs begin to ramp up blood production in the FBM. This reasoning - in combination with our discovery that MPP2s are the primary HSPC subset present in the FBM-led us to test FBM MPP2s for their functional properties, as immunophenotype is not necessarily indicative of function.

MPP2s are a myeloid-biased population of MPPs downstream of LT- and STHSCs and display a relatively high frequency of megakaryocyte/erythroid colonies when plated in CFU-assays (Pietras 2015). To determine the absolute CFU-potential of FBM MPP2s compared to other HSPC sources, we sorted single immunophenotypic MPP2s 


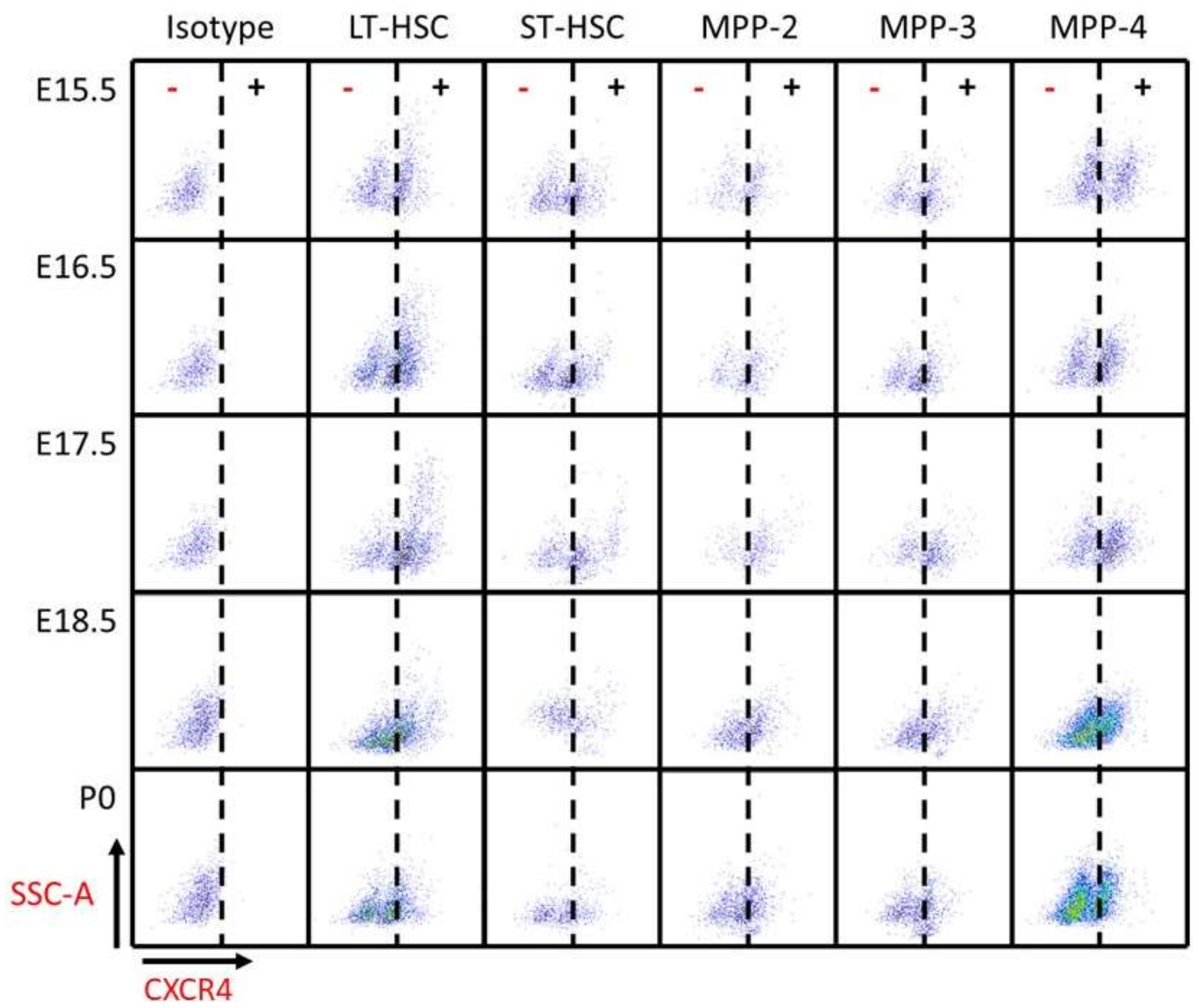

Figure 3-20. FL HSPC CXCR4 cell surface expression

Representative flow cytometry plots depicting CXCR4 cell surface expression on FL HSPCs from E15.5-P0 


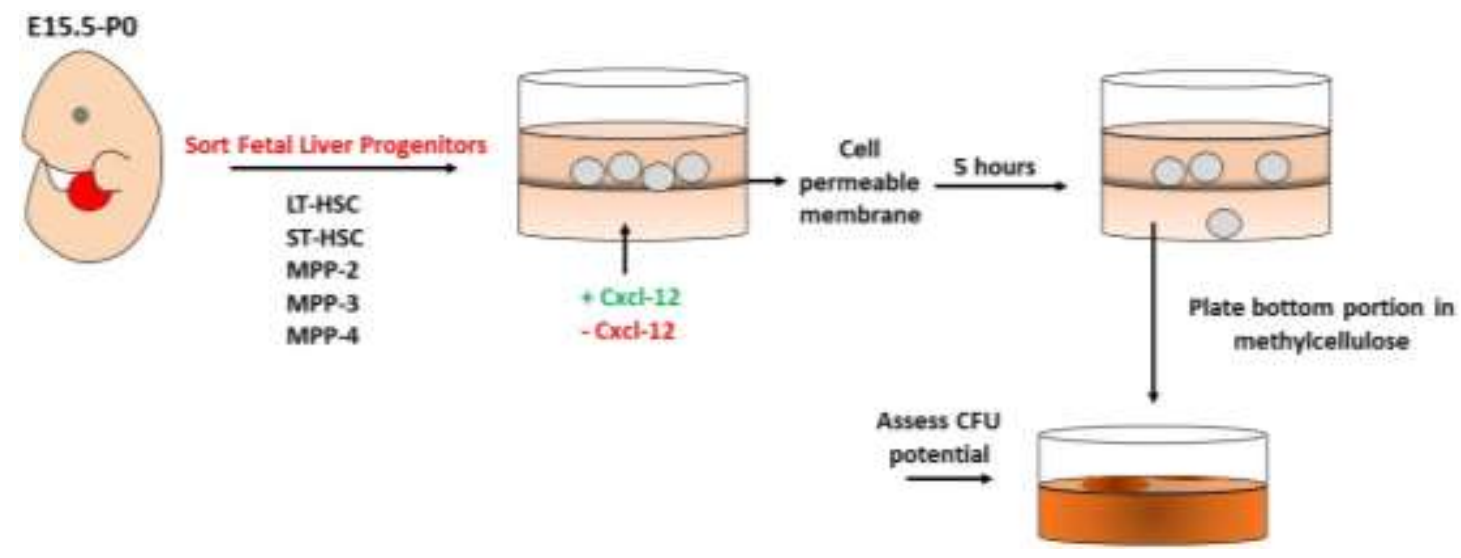

Figure 3-21. Transwell migration assays

FL HSPCs were sorted from E15.5-P0 murine embryos and plated into the top portion of transwell plates, while the bottom portion of the plates contained media with soluble Cxcl-12 or cytokine-free media. After 5 hours, the bottom portion of the transwell was plated in methylcellulose to test for CFU potential.

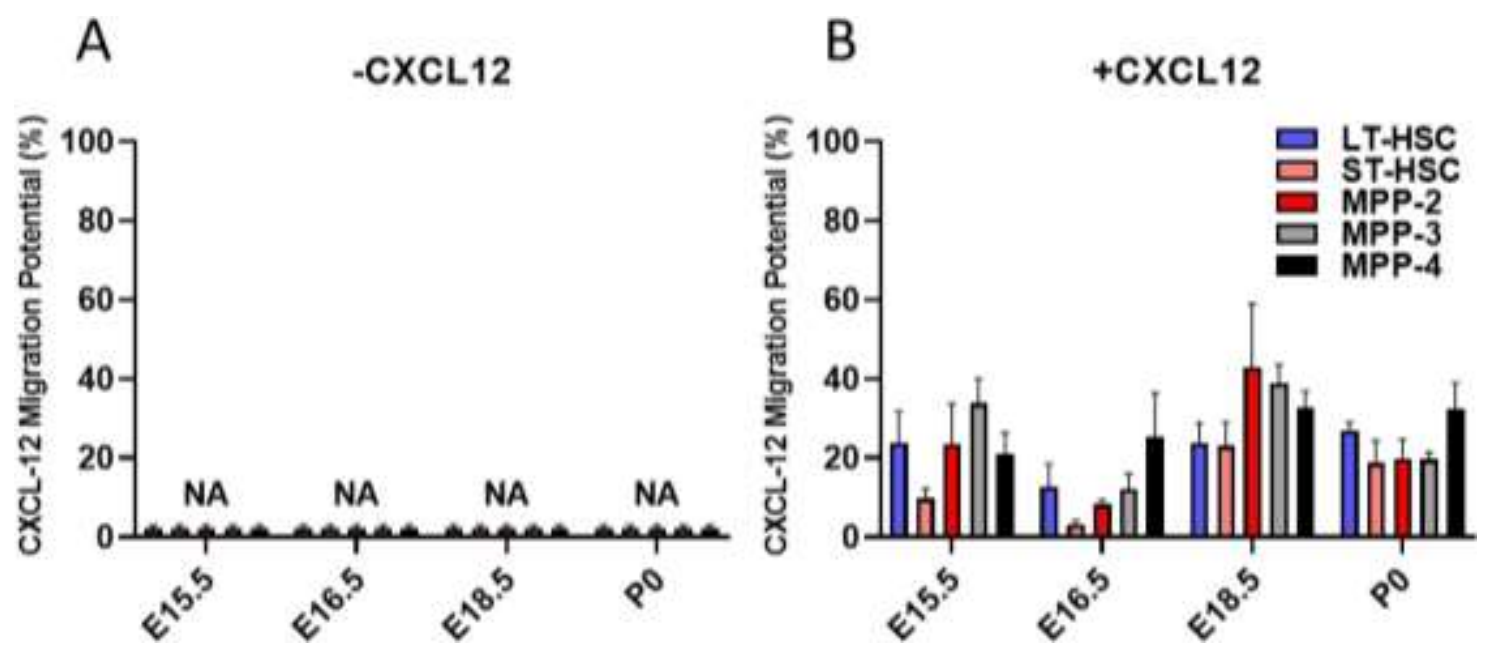

Figure 3-22. FL HSPCs respond to CXCL12

The migration potential of fetal and newborn FL HPSCs plated in transwell assays in the presence of (A) cytokine-free media or $(B)$ soluble CXCL-12 $(n=3)$. All data represent means with SE. 



Figure 3-23. Frequencies of $\mathrm{CD}^{+} 5^{+}$and $\mathrm{CD}^{4} 5^{+} \mathrm{HSPC} \mathrm{BM}$

The frequency of (A) $\mathrm{CD}^{+} 5^{+}$cells in the bone marrow or (B) several HSPC populations in $\mathrm{CD}_{4} 5^{+} \mathrm{BM}$ from C57B6/J embryos and neonates $(\mathrm{n}=10-16$ embryos from 2-3 independent litters). All data represent means with SE. 
from E16.5, E17.5, E18.5, and P0 BM and liver, as well as adult BM as controls, into methylcellulose to assess the frequency and differentiation potential of CFU from the MPP2 compartment (Figure 3-24A). Incredibly, we found that MPP2s from E16.5 FBM displayed no CFU potential, while E17.5 and E18.5 FBM MPP2s had CFU frequencies of 0.01 and 0.03 , respectively (Figure 3-24B). The first appearance of MPP $2 \mathrm{~s}$ with the ability to produce substantial CFUs was right after birth at P0, in which there was a 10fold expansion of CFU frequency compared to E18.5 (Figure 3-24B). Importantly, the frequency of CFU in P0 MPP2s was similar to the CFU frequency in adult BM MPP $2 \mathrm{~s}$, and both MPP2 sources displayed high levels of colonies with megakaryocyte and erythroid potential (Figure 3-24B). Meanwhile, liver MPP2s displayed high CFU frequencies from E16.5-P0, ranging from 0.8 at E17.5 to 0.6 at P0 (Figure 3-24B). These data show that while MPP2s in the FBM are immunophenotypically similar to FL MPP2s and are presumably seeded from the FL, they have distinct in vitro colony-forming potential compared to their FL counterparts at the same developmental timepoints. Likewise, the first robustly functional FBM MPP2s appear to arise around birth, concomitant with the shift in HSPC frequencies we previously observed (Figures 3-19 and 3-23).

Upon transplantation, MPP2s will transiently repopulate irradiated hosts with a bias towards myeloid, platelet, and erythrocyte production (Pietras et al.). To assess the functional potential of FBM MPP2s across late embryonic ontogeny, we isolated MPP2s from E16.5, E18.5, and P0 BM and liver, as well as adult BM, and transplanted a limiting dose of cells (100 cells) into lethally irradiated recipients (Figure 3-25). Erythrocytes and platelets do not express cell surface CD45, therefore we utilized $U B C$-GFP mice, which express a green fluorescent protein (GFP) transgene under the control of the human Ubiquitin $C$ (UBC) promoter in all blood cell types, to track the contribution of MPP2 cells to myeloid, B, T, platelet, and erythroid lineages in the PB of recipients (Schaefer 2001) (Figure 3-25). As expected, adult MPP2s were able to transiently reconstitute $50 \%$ of irradiated recipients with combinations of myeloid, T-lymphoid, platelet, and erythrocyte lineages, with a greater percentage of recipients showing myeloid, platelet, and erythrocyte donor contribution compared to T-lymphoid reconstitution (Figures 3-26A and 3-27). Similar to our in vitro results, E16.5 FBM MPP2s were unable to transiently reconstitute any lineages in the PB of irradiated recipients while E18.5 was the first time point in which transplantable MPP2s were found, albeit at low levels (Figures 3-26A and 3-27). Indeed, E18.5 FBM MPP2s were able to transiently reconstitute $20 \%$ of recipients with combinations of platelet, erythroid, and even B-lymphoid lineages (Figures 3-26A and 3-27). Meanwhile, recipients transplanted with P0 FBM MPP2s showed $40 \%$ transient reconstitution of all lineages except T-lymphoid, although donor chimerism in these lineages was lower than in recipients transplanted with adult BM (Figures 3-26A and 3-27). Also similar to our in vitro CFU assay results, the first FL MPP2 cells displaying transient reconstitution of multiple lineages were from E16.5 FL, and E16.5 and E18.5 FL MPP2s displayed transient reconstitution of all lineages-but preferentially myeloid, platelet, and erythrocyte - in $67 \%$ and $83 \%$ of recipients, respectively (Figures 3-26B and 3-27). Interestingly, there was a drop in the percentage of recipients that were transiently reconstituted with P0 FL MPP2s to 40\%, and these MPP2s displayed contribution to all lineages except the myeloid lineage (Figures 3-26B 

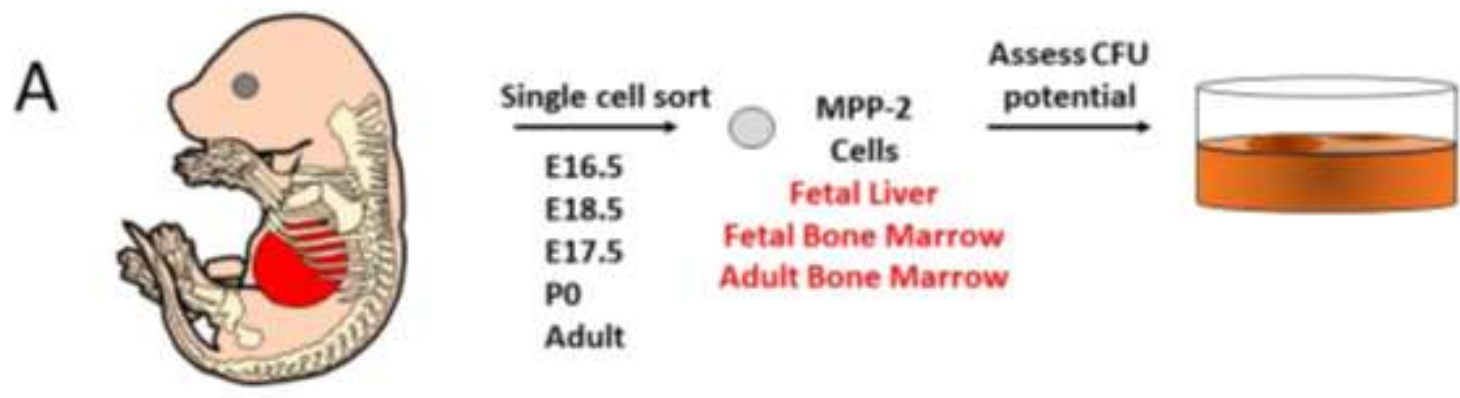

E17.5 Fetal Bone Marrow

Po

Adult Bone Marrow

Adult

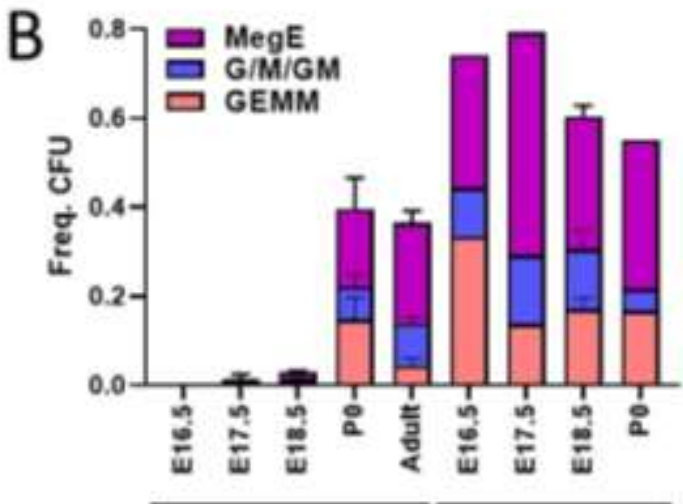

Bone Marrow

Liver

Figure 3-24. In vitro assay for MPP2 function

A. Single MPP2s from FL, FBM, newborn BM/liver, and adult BM were isolated and plated directly into cytokine-supported methylcellulose to test for CFU potential. B. The frequency of CFUs among MPP2s from BM and liver at different developmental timepoints ( $\mathrm{n}=58-169$ single cells from 1-3 independent experiments). Data represent means with SE. 


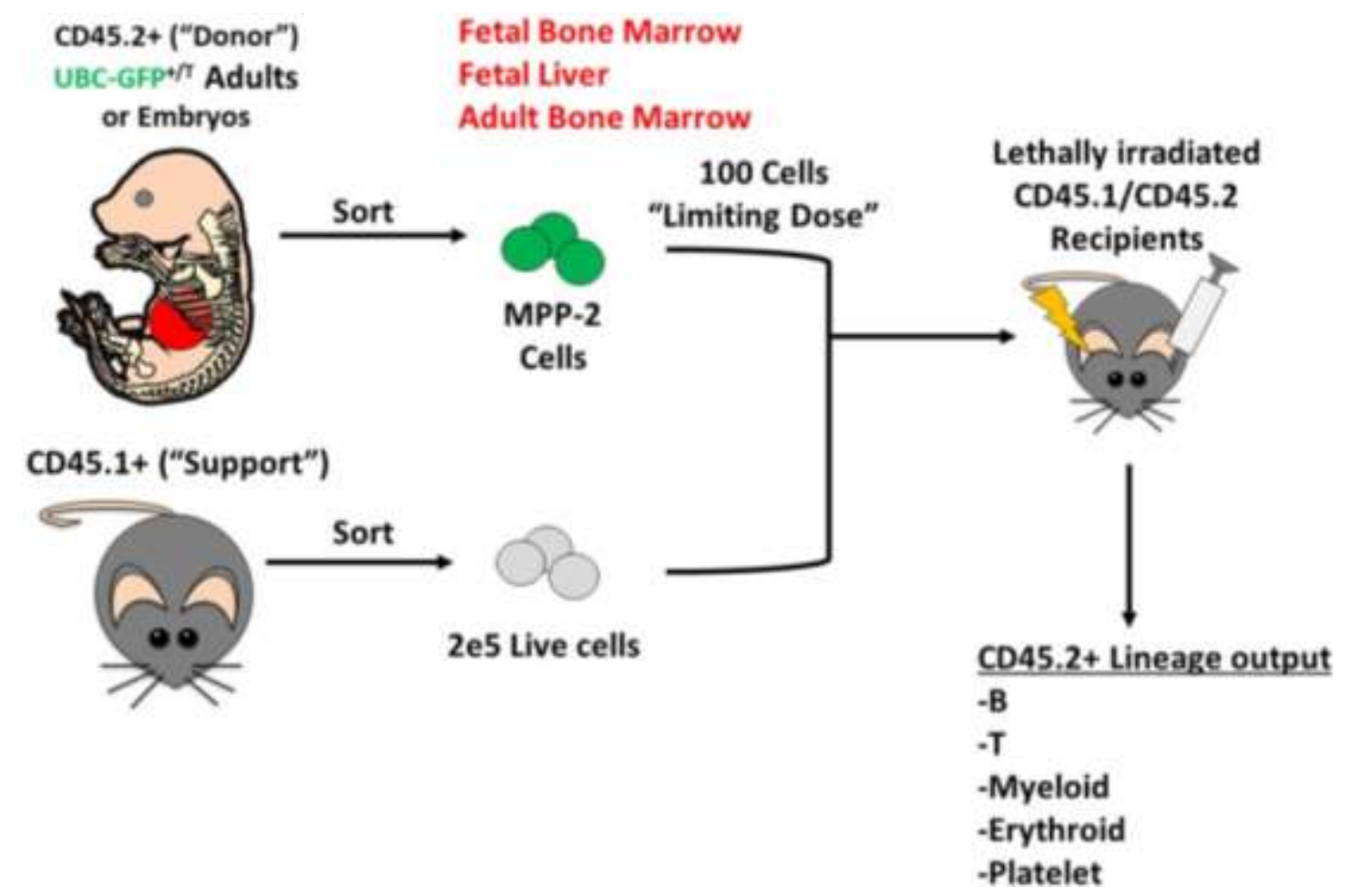

Figure 3-25. In vivo assay for MPP2 function

Experimental schematic for in vivo analysis of MPP2 cell function. $100 \mathrm{MPP} 2 \mathrm{~s}$ were sorted from $\mathrm{CD} 45.2^{+}$donor UBC-GFP ${ }^{+/ T} \mathrm{FL}, \mathrm{FBM}$, newborn liver/BM, or adult BM and transplanted into lethally irradiated recipients along with $2 \times 10^{5}$ sorted CD45.1 $1^{+}$support cells, and recipients were monitored for donor contribution to B-lymphoid, T-lymphoid, myeloid, erythroid, and platelet production in the PB. 


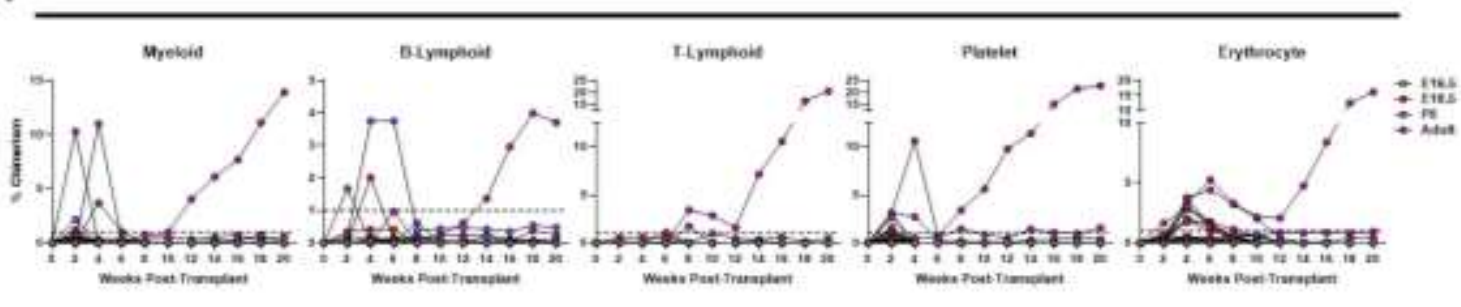

B

Liver

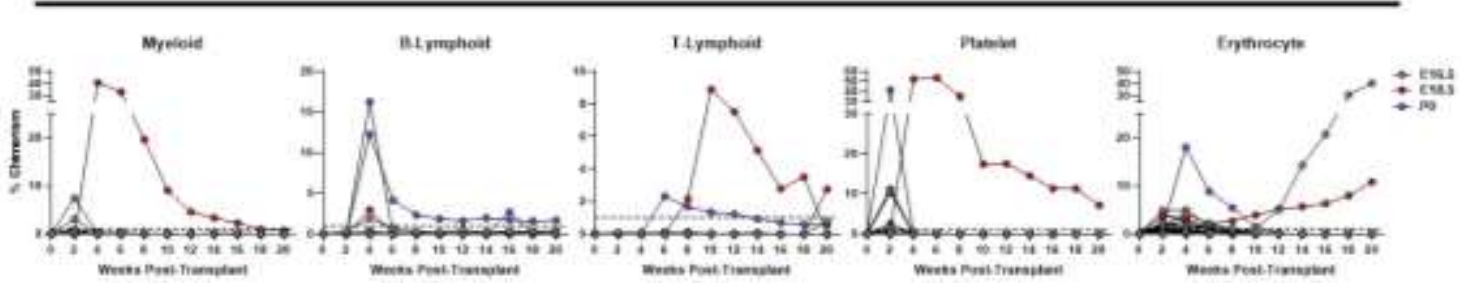

Figure 3-26. FBM MPP2s are inert until E18.5

Donor chimerism of myeloid cells, B-/T-lymphoid cells, platelets, and erythrocytes in recipients transplanted with either (A) BM or (B) liver cells over 20 weeks $(n=5-10$ recipients per timepoint). 


\section{\# Lineages}

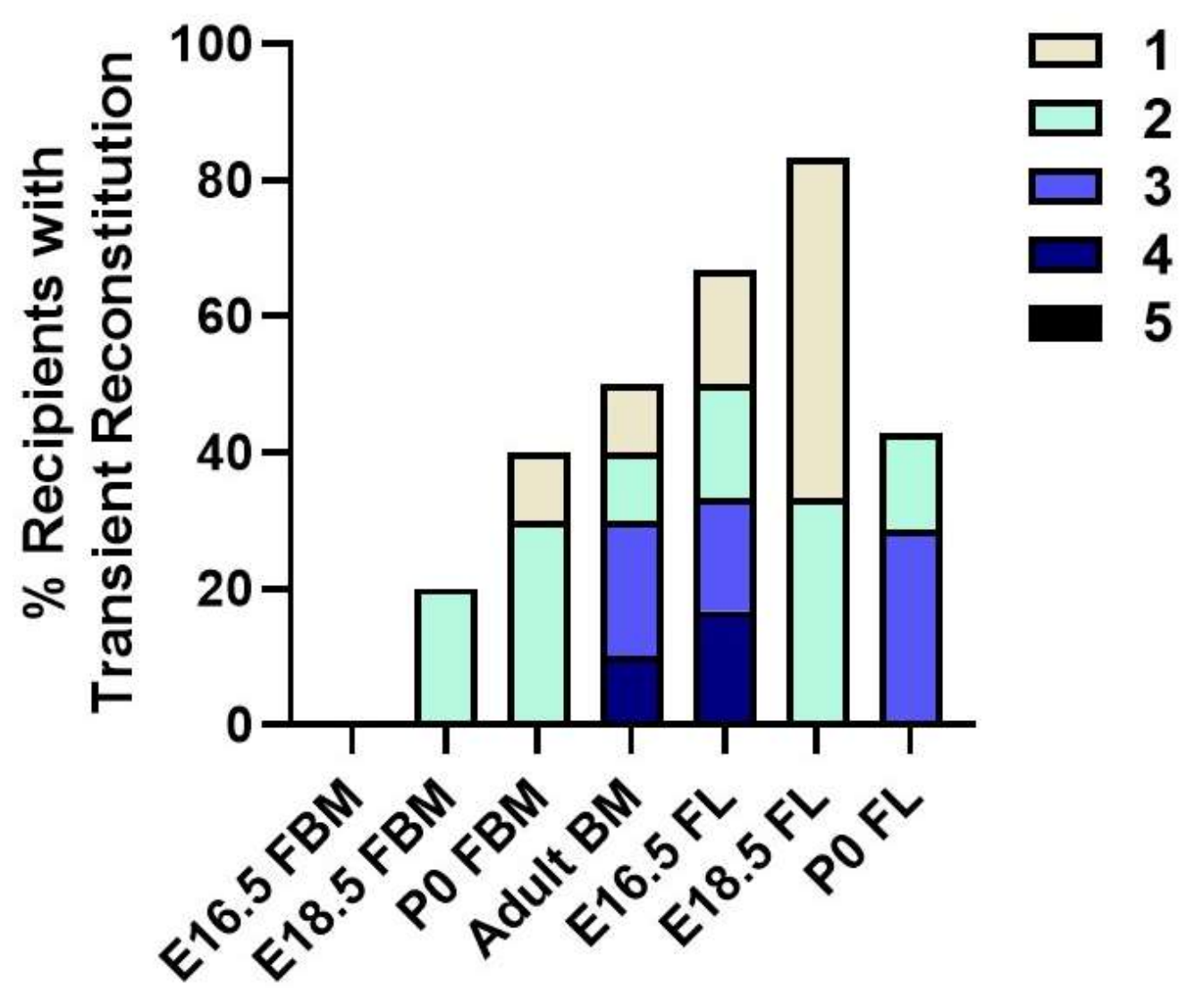

Figure 3-27. Summary of MPP2 transplants

Histogram summarizing the percentage of recipients displaying transient donor reconstitution of varying numbers of lineages after being transplanted with BM or liver from different developmental timepoints $(n=5-10$ recipients $)$. 
and 3-27). Such a dramatic shift was not seen in our in vitro CFU assays, although there was a trend for P0 FL MPP2s to display lower CFU frequency compared to earlier timepoints (Figure 3-24B).

Together, these data unequivocally show that the pool of MPP2 cells in the FBM display increasing functional output leading up to birth, at which point MPP2 cells more closely resemble their adult BM counterparts. Perhaps even more interesting is the observation that MPP2 cells from FBM and FL time-matched tissues display large disparities in their functional output, with FBM MPP2 cells being more "immature" in their functional capacity compared to FL MPP2s. This could possibly be due to an accumulation of more functionally "mature" MPP2s in the FBM during late ontogeny, may be reflective of an unwelcome HSPC niche in the FBM until birth, or could be some combination of these possibilities.

\section{The FBM Hematopoietic Progenitor Compartment Undergoes Drastic Remodeling During Late Gestation}

To better understand why MPP2s and other HSPCs may be functionally distinct early in FBM colonization, we isolated $\mathrm{CD}^{2} 5^{+}$Lineage $^{-} \mathrm{c}-\mathrm{Kit}^{+}$hematopoietic progenitors (HPs) and CD45-Ter119- stroma from E16.5, E18.5, P0, and adult BM, followed by 10X scRNA-Seq analysis (Figure 3-28A). As HSPCs in the FBM are extremely rare, we also utilized CITE-Seq (Stoeckius 2017) to label HSPCs within the HP compartment with oligo-tagged antibodies that could later be combined with mRNA expression data to immunophenotypically identify HSPCs in our 10X-generated scRNA-Seq dataset (Figure 3-28). In total, we constructed and sequenced libraries from 5,985 adult BM, 6,208 P0 BM, 3,477 E18.5 FBM, and 5,312 E16.5 FBM HP cells.

Unbiased clustering and gene expression analysis allowed us to prospectively identify several distinct cell populations in each library, and we primarily relied upon the expression of cell-specific markers and previously published datasets to manually annotate all clusters (Cabezas-Wallscheid 2014, Kowalczyk 2015, Paul 2015, Wilson 2015, Dahlin 2015, de Graf 2016, Nestowara 2016, Dwyer 2016, Velten 2017, Chhiba 2017, Dahlin 2018, Giladi 2018, Rodriguez-Fraticelli 2018, Tusi 2018, Zheng 2018, Pellin 2019, Baccin 2019) (Figure 3-29). We also utilized Uniform Manifold Approximation and Projection (UMAP) (McInnes 2018) to produce dimensionality maps reflecting similar expression relationships between clusters. Indeed, UMAP dimensionality reduction on annotated clusters from the adult HP library placed several pre/pro-B cell and B-cell lineage clusters in close proximity, as well as placing an HSPC (Prog) population at the apex of monocyte/granulocyte and megakaryocyte/erythroid lineages, suggesting that our method for annotating clusters was robust (Figure 3-29A).

Adult, P0, and E18.5 BM libraries contained similar populations, including progenitors, several granulocyte and monocyte clusters, mast cell clusters, megakaryocyte/erythroid progenitors, and B-cell clusters (Figure 3-29A-C). The most apparent differences between E18.5/P0 BM and adult BM were the lack of pre/pro-B-cell 


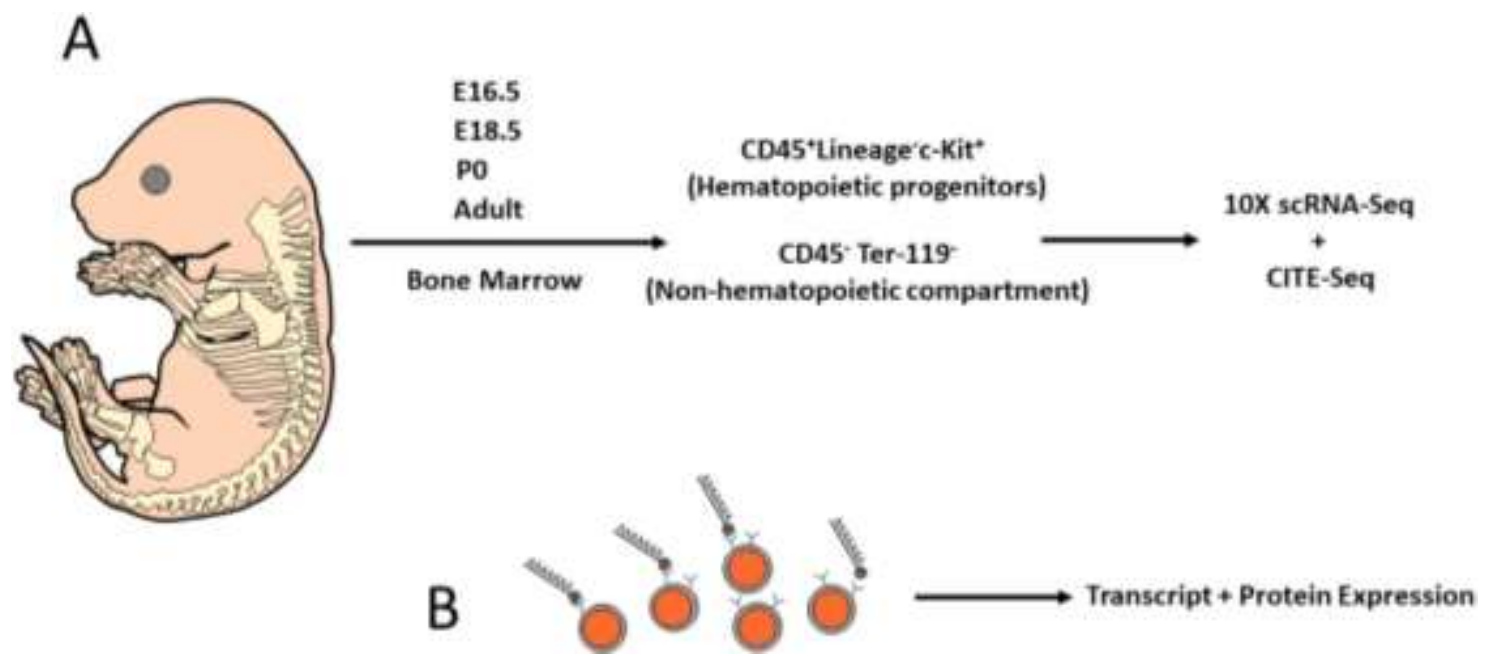

Figure 3-28. Single cell RNA-sequencing of FBM

Schematic for the isolation and 10X scRNA-Seq of BM hematopoietic progenitors (HP) or stromal cells. A. BM was isolated from E16.5, E18.5, P0, and adult C57B6/J mice and sorted for hematopoietic progenitors $\left(\mathrm{CD}^{4} 5^{+} \mathrm{Lineage}^{-} \mathrm{c}-\mathrm{Kit}^{+}\right)$or stromal cells $\left(\mathrm{CD} 45^{-} \mathrm{Ter}-\right.$ $119^{-}$), followed by $10 \mathrm{X}$ scRNA-Seq in conjunction with CITE-Seq. B. CITE-Seq utilizes oligo-tagged antibodies containing specific barcodes that can be captured by the $10 \mathrm{X}$ technology, allowing parallel sequencing of mRNA expression and cell-surface protein expression of single cells. 

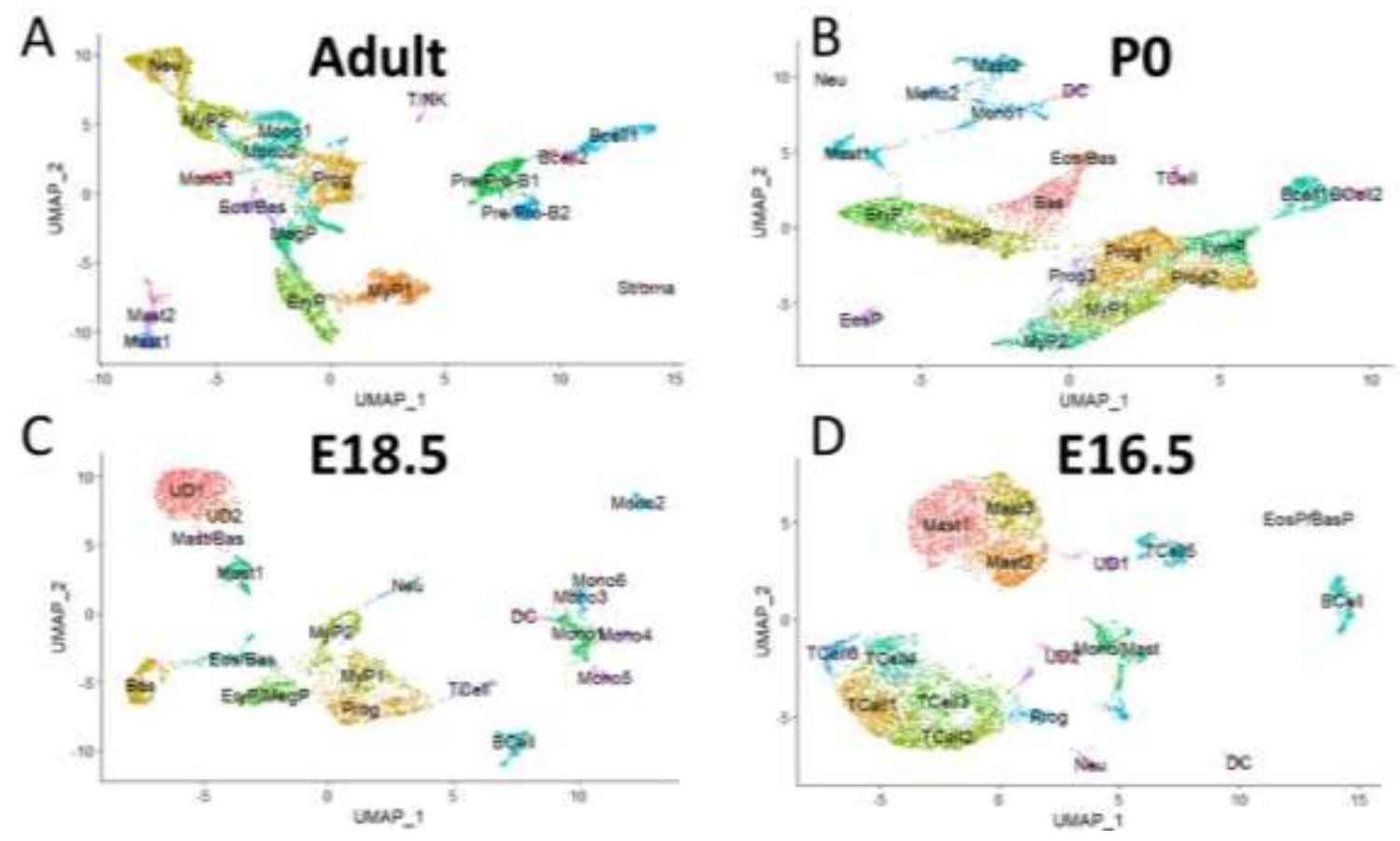

Figure 3-29. Hematopoietic clusters in FBM scRNA-Seq data

UMAP projections depicting the cell clusters of HP libraries from (A) adult, (B) P0, (C) E18.5, and (D) E16.5 BM. Bas, basophil lineage; Bcell, B-cell lineage; DC, Dendritic cells; Eos, eosinophil lineage; EryP, erythroid progenitors; Mast, mast cell lineage; MegP, megakaryocyte progenitors; Mono, monocyte lineage; MyP, myeloid progenitors; Neu, neutrophil lineage; Pre/Pro-B, Pre/Pro-B Cells; Prog, HSPCs; TCell, T-Cell lineage; T/NK, T/NK cell lineage; UD, undefined cluster. 
clusters in E18.5/P0 BM, as well as a large expansion of the neutrophil cluster in adult BM (Figure 3-29A-C). There was also a greater presence of progenitor cells in the P0 BM compared to adult and E18.5 BM, suggesting an expansion of this population around birth (Figure 3-29A-C). However, the most striking observation was the presence of several large mast and T-cell lineage clusters in the E16.5 FBM (Figure 3-29D). Within the E16.5 FBM HP library, mast cell and T-cell clusters comprised $38.8 \%$ and $40.6 \%$ of all cells, respectively (Figure 3-29D). Meanwhile, T-cell clusters comprised only 1.1$1.8 \%$ of cells from all other libraries, and mast cell clusters comprised only $4.7-8.6 \%$ of cells (Figure 3-29). Our E16.5 FBM HP library also lacked any definable myeloid, erythroid, megakaryocyte, or lymphoid progenitors, but did contain a small population of undefined progenitor cells (Figure 3-29D). Therefore, the HP compartment of E16.5 FBM is heavily biased towards the mast cell and T-cell lineages, which are rapidly reduced by E18.5 followed by an expected expansion of progenitor cells right after birth (Figure 3-29).

\section{Immunophenotypic MPP2s Display Highly Heterogeneous Transcriptional Programs Compared to LT-HSCs}

By incorporating CITE-Seq (Stoeckius 2017) into our 10X pipeline, we were able to identify the same immunophenotypic populations we previously assayed for our functional studies in our scRNA-Seq dataset (Figure 3-30A). While cell-surface protein expression does not always mimic mRNA expression, we were able to compare our CITE-Seq expression data with corresponding mRNA expression to verify that our CITESeq antibodies labelled similar cell populations (Figure 3-30B). To determine which clusters MPP2 cells preferentially resided in, we identified immunophenotypic MPP2s using CITE-Seq expression and calculated the proportions of MPP2s in the clusters of each library (Figure 3-31).

Immunophenotypic MPP2s could be found in 15/18 clusters in the adult BM HP library (Figure 3-31A). The HSPC (Prog) and megakaryocyte progenitor clusters compromised $18 \%$ and $15 \%$ of immunophenotypic MPP2s, but surprisingly, $27 \%$ of immunophenotypic MPP2s fell into the cluster we annotated as "Bcell1" due to its expression of several B-lineage markers (Figure 3-31A). Likewise, 16/20 clusters from our P0 BM HP library contained MPP2s, with 74\% of MPP2s falling into the monocyte1, Prog-1, Prog-2, and megakaryocyte progenitor clusters (Figure 3-31B). Displaying slightly less heterogeneity, E18.5 BM MPP2s fell into 11/20 clusters and were found primarily in the Prog, myeloid progenitor-1, B-lineage, and monocyte-3 clusters, although other clusters such as more monocyte, erythroid/megakaryocyte progenitors, and T-cell lineage clusters each contained $>5 \%$ of immunophenotypic MPP $2 \mathrm{~s}$ at this time point (Figure 3-31C). Finally, our E16.5 FBM HP library contained MPP2s with similar heterogeneity compared to E18.5 FBM, with 9/17 clusters containing MPP2s (Figure 3-31D). However, at E16.5 53\% of immunophenotypic MPP2s were contained within the monocyte/mast cell cluster, suggesting that the greatest enrichment of homogeneous MPP2s from our dataset is in the E16.5 FBM (Figure 3-31D). 

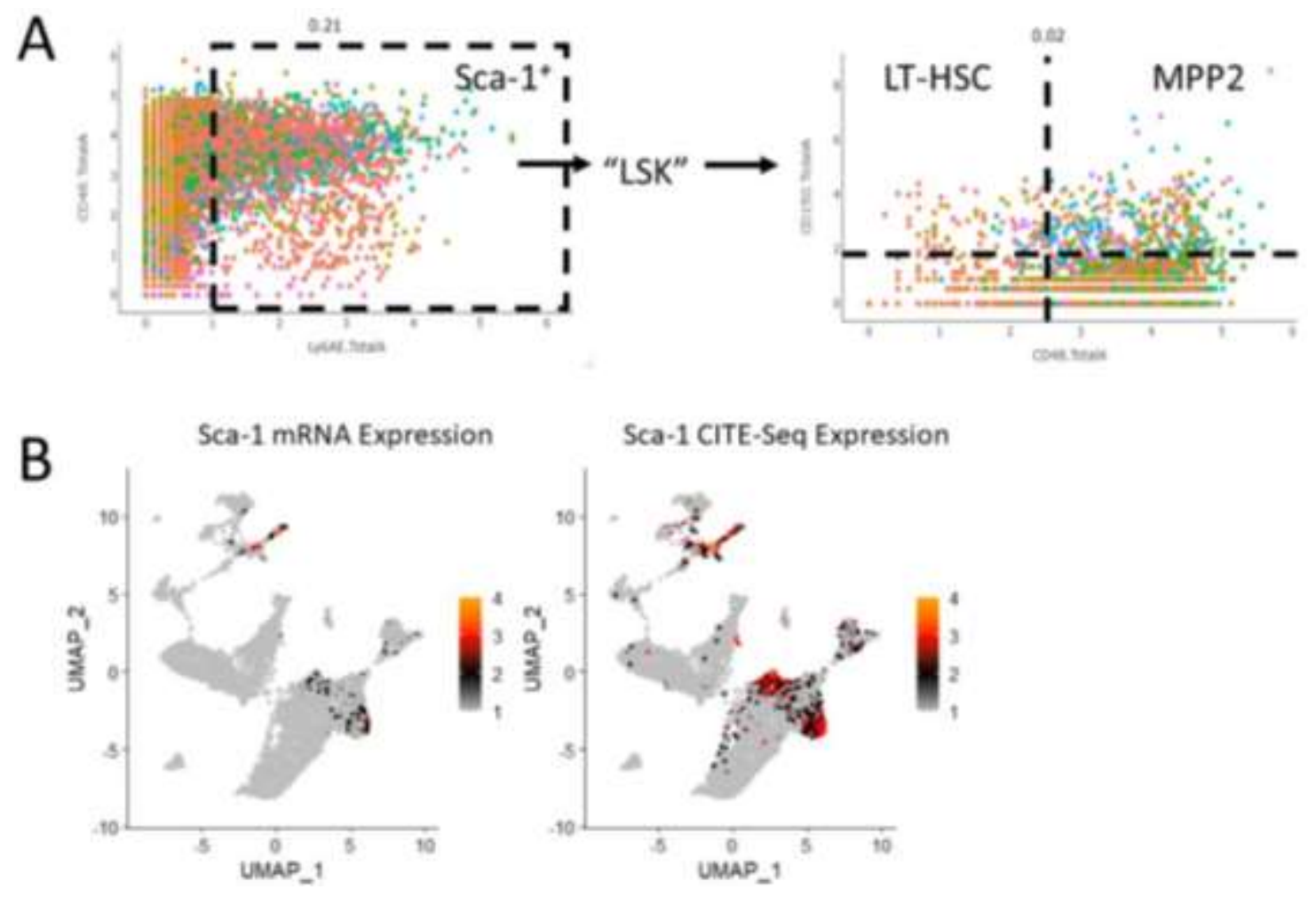

Figure 3-30. CITE-Seq to identify HSPCs

CITE-Seq can be used to prospectively identify immunophenotypic HSPCs. A. CITE-Seq expression dot plots for identification of immunophenotypic LT-HSCs and MPP2s. B. UMAP projection of P0 BM depicting Sca-1 mRNA and CITE-Seq expression. 


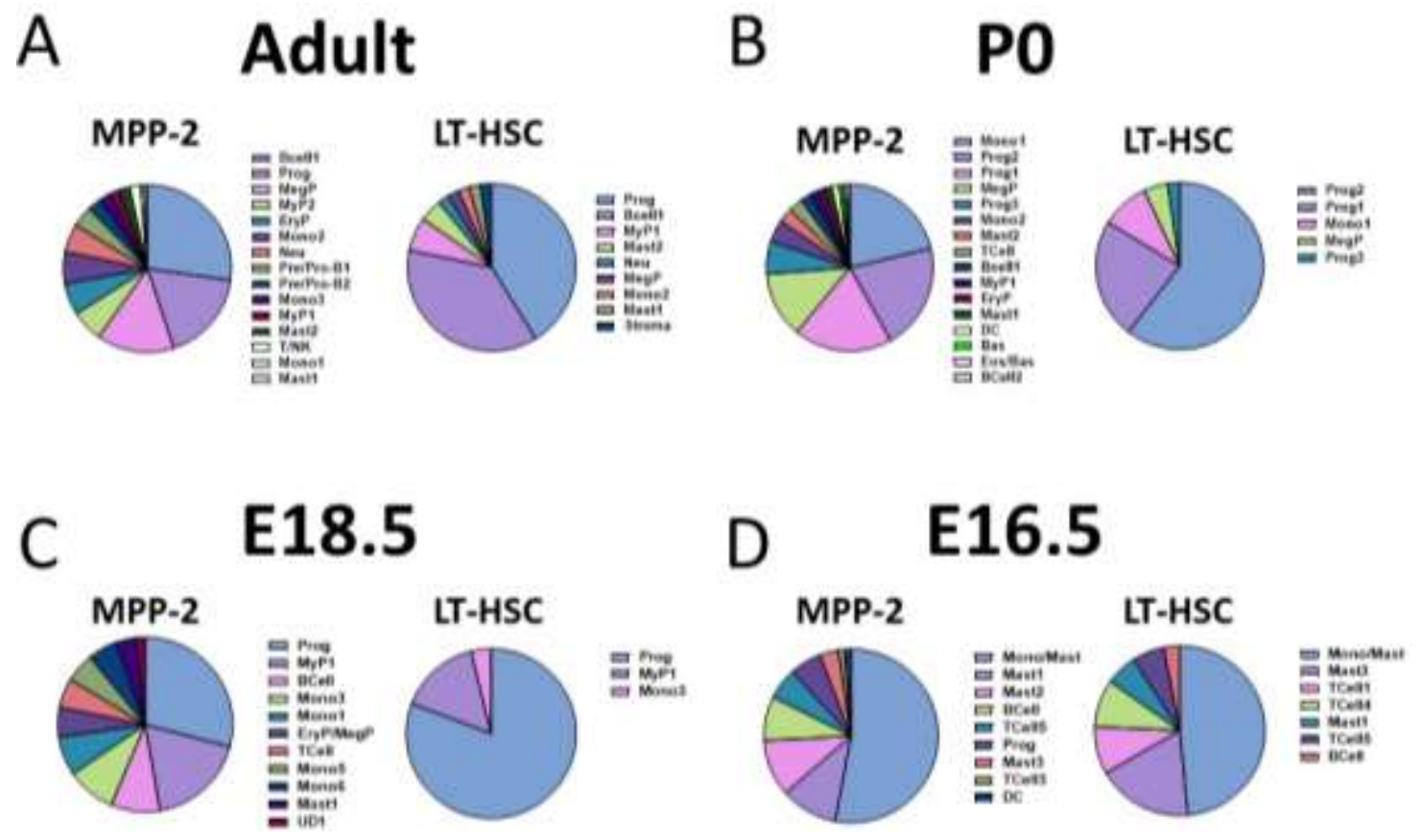

Figure 3-31. Cluster distribution of HSPCs

Circle graphs depicting the distribution of immunophenotypic MPP2s or LT-HSCs contained within each cell cluster of (A) adult, (B) P0, (C) E18.5, or (D) E16.5 HP libraries. 
Given previous observations that MPP2s are a highly heterogeneous population (Pietras 2015), we also used our CITE-Seq data to identify LT-HSCs and we hypothesized they would contain less heterogeneity than MPP2s. Immunophenotypic LTHSCs could be found in 9/18 clusters in our adult BM HP library, but 78\% of LT-HSCs were only found in the Prog and Bcell1 clusters (Figure 3-31A). This adult Bcell1 cluster either represents a B-lymphoid-biased progenitor population-as both immunophenotypic LT-HSCs and MPP2s are found at high levels in this cluster-or perhaps contains non-functional immunophenotypic progenitors. Meanwhile, only 5/20 clusters in our P0 BM HP library contained immunophenotypic LT-HSCs, and 83\% fell into the Prog-1 and Prog-2 clusters (Figure 3-31B). Similarly, low heterogeneity was seen in E18.5 immunophenotypic LT-HSCs, as they only fell into 3/20 clusters, with 81\% of LT-HSCs residing in the Prog cluster (Figure 3-31C). Finally, there was slightly more heterogeneity present in E16.5 LT-HSCs, as 7/17 clusters contained immunophenotypic LT-HSCs (Figure 3-31D). However, the majority of these cells also fell into the same monocyte/mast cell cluster as the MPP2s, suggesting that this cluster contains the majority of all immunophenotypic HSPCs (Figure 3-31D).

Together, these analyses confirm that MPP2s are a highly heterogenous population of cells, but also suggest that the majority of immunophenotypic MPP2s contain a monocyte/mast cell transcriptional signature at E16.5, shifting to a signature more indicative of myeloid and megakaryocyte/erythroid progenitors by E18.5. Meanwhile, immunophenotypic LT-HSCs appear to be more heterogeneous at E16.5 and are primarily composed of cells with monocyte/mast cell transcriptional signatures, suggesting that the LT-HSC compartment in the early FBM may have reduced HSC function.

\section{The Stem Cell-Specific Transcriptional Profiles of Immunophenotypic LT-HSCs and MPP2s Shift Around Birth}

Recently, the Göttgens group developed the "hscScore" algorithm to assist researchers in identifying LT-HSCs and downstream progenitors in scRNA-Seq datasets (Hamey 2019). This algorithm assigns a score to each cell based on the expression of genes validated to enrich for LT-HSCs (Wilson 2016). For reference, applying this algorithm to previous scRNA-Seq datasets showed that the most enriched HSC populations have an hscScore around 0.4-0.5, while MPP populations typically have an hscScore ranging from 0.1-0.3 (Hamey 2019). Given that our MPP2 functional assays showed that FBM MPP2s do not become functional until right before birth and that our CITE-Seq assisted scRNA-Seq analyses placed E16.5 immunophenotypic LT-HSCs primarily in a cluster with monocyte/mast cell gene expression signatures, we utilized the hscScore algorithm to identify changes in the stem cell signature of MPP2s and LT-HSCs across FBM ontogeny.

We subclustered our immunophenotypic MPP2s and LT-HSCs and generated an hscScore for subclusters at each timepoint (Figures 3-32 and 3-33). While the majority of immunophenotypic MPP2s displayed hscScores $<0.1$ across development, at E18.5 and 


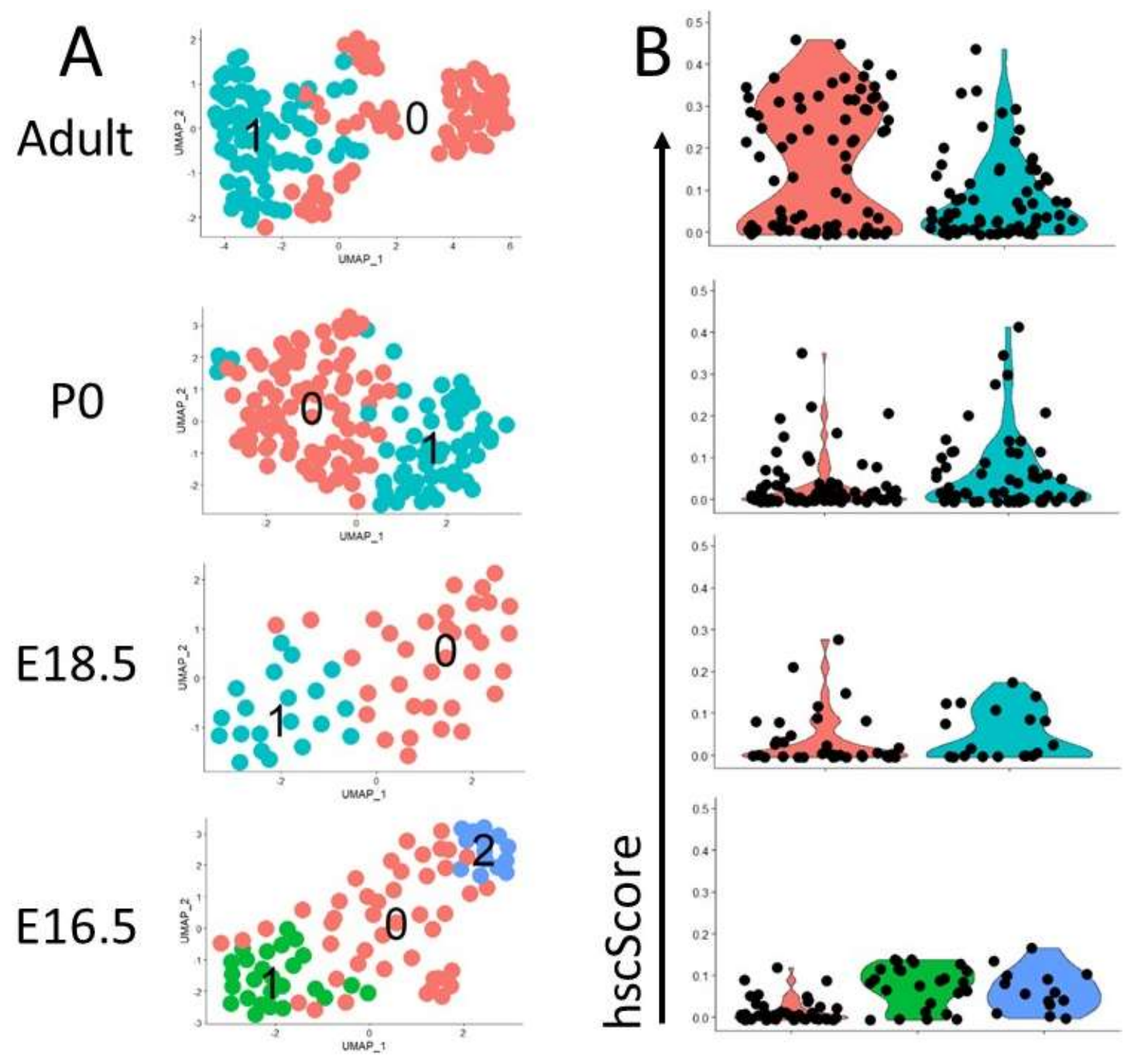

Figure 3-32. MPP2 hscScores across ontogeny

hscScore was used to quantify stem cell associated transcriptional programs in MPP2 cells across BM ontogeny. A. Subclustering of immunophenotypic MPP2 cells from each HP library. B. hscScore for the representative subclusters at each developmental timepoint. 


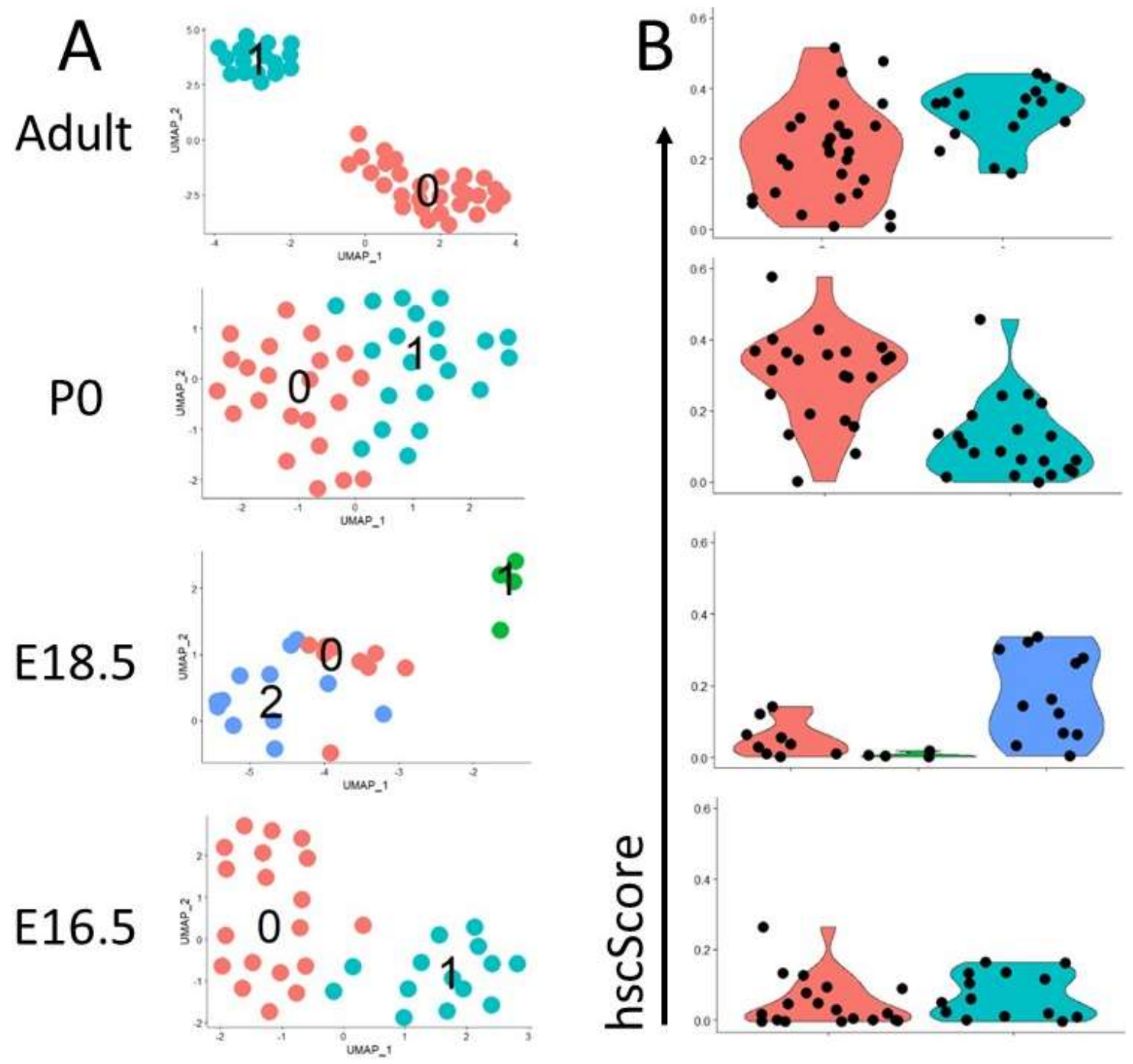

Figure 3-33. LT-HSC hscScores across ontogeny

hscScore was used to quantify stem cell associated transcriptional programs in LT-HSC cells across BM ontogeny. A. Subclustering of immunophenotypic LT-HSC cells from each HP library. B. hscScore for the representative subclusters at each developmental timepoint. 
P0 there was a gradual increase in the number of MPP2s with hscScores $>0.15$, and adult immunophenotypic MPP2s contained cells with hscScores ranging from 0.15-0.45

(Figure 3-32). This provides further evidence that the MPP2 compartment during early FBM ontogeny is primarily composed of cells lacking the necessary transcriptional programs that are indicative of functional progenitors.

The immunophenotypic LT-HSC compartment in the FBM also displayed interesting dynamics from E16.5-P0. At E16.5, the majority of LT-HSCs consisted of hscScores $<0.2$, suggesting that at this timepoint many LT-HSCs may not function properly (Figure 3-33). By E18.5, one subcluster of LT-HSCs was already displaying hscScores greater than 0.35 while two other subclusters had hscScores $<0.2$ (Figure 3-33). In the BM of newborn mice, hscScore ${ }^{\text {hi }}$ and hscScore ${ }^{\text {lo }}$ LT-HSC subsets existed, with the "hi" subset being comprised of cells with scores around 0.4 and the "lo" subset consisting of cells ranging from $\sim 0.1-0.3$ (Figure 3-33). Finally, the adult BM contained LT-HSCs with hscScore $^{\text {hi }}(\sim 0.4)$ and hscScore ${ }^{\text {mid }}(\sim 0.3)$ subclusters (Figure 3-33).

In sum, the hscScore values provide clues to the maturation process of MPP2s and LT-HSCs in the FBM and suggest that these progenitor cells can undergo dramatic transcriptional changes from E16.5 to $\mathrm{P} 0$ in favor of a phenotype more indicative of functional progenitors.

\section{The FBM Stromal Compartment Contains Putative Niche Cells for HSPCs}

Our observation that immunophenotypic MPP2s and LT-HSCs appear to accumulate transcriptional programs necessary for stem cell identify during late gestation suggests that changes in the FBM niche around birth may play a role in this maturation process. Therefore, we isolated CD45-Ter1 19- ${ }^{-}$stroma from E16.5, E18.5, P0, and adult $\mathrm{BM}$, followed by 10X scRNA-Seq analysis to visualize changes in the cellular composition of the FBM stroma across development (Figure 3-28A). In total, we constructed and sequenced libraries from 7,380 adult BM, 16,412 P0 BM, 7,272 E18.5 FBM, and 9,786 E16.5 FBM stromal cells.

Similar to our analysis of the HP compartment, we utilized unbiased clustering based on gene expression to prospectively identify several cell populations in each library, relying upon the expression of cell-specific markers and previously published datasets to manually annotate all clusters (Cai 2017, Baccin 2019, Mende 2019, Severe 2019, Baryawno 2019, Mende 2019, Tikhonova 2019, Wolock 2019) (Figure 3-34). Again, we utilized UMAP (McInnes 2018) to produce dimensionality maps reflecting

similar expression relationships between clusters. Indeed, UMAP dimensionality reduction on annotated clusters from the adult stromal library placed several fibroblast and chondrocyte clusters in close proximity, as well as the arterial and sinusoidal endothelial cells (Figure 3-34A).

Across development, several chondrocyte and fibroblast clusters were detected, as well as many pericyte clusters, including clusters with transcriptional signatures for 

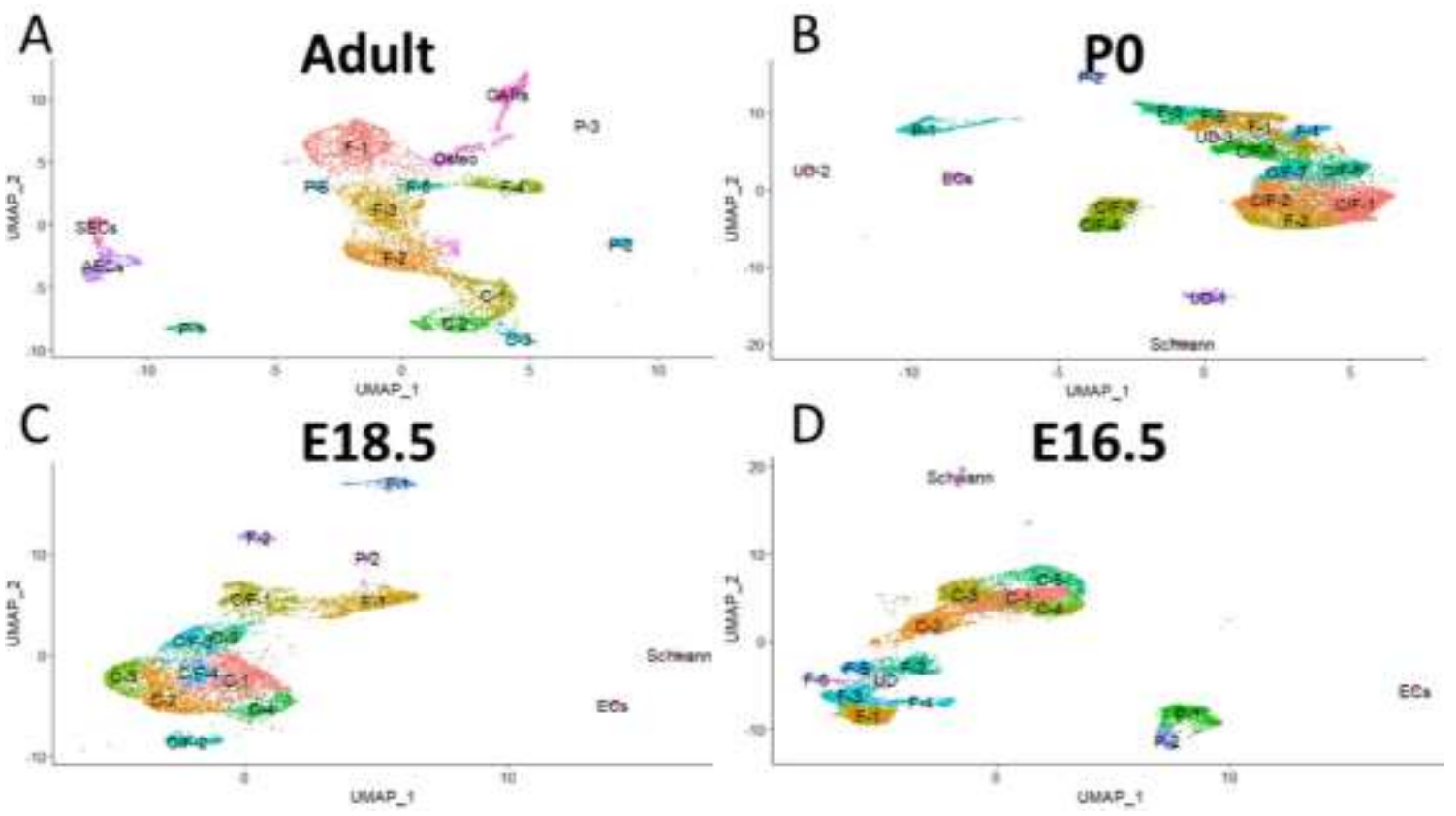

Figure 3-34. Stromal clusters in FBM scRNA-Seq data

UMAP projections depicting the cell clusters of stroma libraries from (A) adult, (B) P0, (C) E18.5, and (D) E16.5 BM. CARs, CXCL12-abundant reticular cells; C, chondrocytes; C/F, chondrocyte/fibroblast; ECs, endothelial cells; AECs, arterial endothelial cells; SECs, sinusoidal endothelial cells; F, fibroblasts; Osteo, osteolineage; P, pericytes; Schwann, schwann cells; UD, undefined cluster. 
smooth muscle cells, Schwann cells, and myofibroblasts (all of which were considered "pericyte" clusters) (Figure 3-34). However, there were a few key differences between the fetal/neonatal stromal composition and the adult stroma. For instance, no clear osteolineage population could be annotated in E16.5 to P0 stroma, only clusters which contained gene signatures for osteoblasts in addition to chondrocyte or fibroblast markers (Figure 3-34). In addition, the presence of several chondrocyte/fibroblast clusters - in which transcripts for both cell types were highly upregulated-were enriched in the E16.5-P0 BM stroma libraries, suggesting that the stroma of the fetal/neonatal stroma has a higher composition of mesenchymal progenitor or multipotent cells (Figure 3-34). Sinusoidal and arterial endothelial cells could not be distinguished in E16.5-P0 BM as in adult BM, although this does not preclude their existence, and may simply be due to the reduction in total endothelial cell numbers in the fetal/neonatal BM (Figure 3-34). Most importantly, the presence of a clear CAR cell cluster was absent from E16.5-P0 BM, suggesting that this critical adult BM HSC niche cell is only substantially present after P0 (Figure 3-34).

CAR cells are a well-documented population of cells that regulate HSC maintenance in the adult BM, and express high levels of Cxcl12 and Kitl (Crane 2017, Gao 2018). Since we could not accurately annotate a CAR cell population in E16.5-P0 BM, we assessed the Cxcl12 and Kitl expression among all clusters from these time points to determine putative cell populations for HSC maintenance (Figures 3-35 and 3-36). As expected, the CAR cluster in our adult BM stroma library was highly enriched for Cxcl12 expression, as were arterial endothelial cells - one of the proposed foundational niche cells where many CARs and HSCs reside (Figure 3-35A). The adult CAR cluster was also enriched for Kitl expressing cells, along with arterial endothelial cells and a cluster of pericytes that closely resemble smooth muscle (P-1) (Figure 3-36A).

Meanwhile, Cxcl12 and Kitl expression was more broadly distributed and expressed at lower levels in E16.5-P0 BM stroma libraries (Figures 3-35 and 3-36). The three clusters with the highest $\mathrm{Cxcl12}$ expression in P0 stroma were an undefined cluster (UD-3; with some CAR and fibroblast/osteoblast signatures), a fibroblast cluster (F-3, displaying potential adipocyte signatures), and a pericyte cluster (P-2, enriched for smooth muscle signatures, as well as a few adipocyte signatures) (Figure 3-35B). Interestingly, F-3 and P-2 were also enriched for Kitl among P0 clusters, suggesting that these cell populations may be important niche cells for P0 HSCs (Figure 3-36B). However, it is important to note that the level of Cxcl12 and Kitl in these clusters was much lower than the adult CAR cluster. Inspection of the top Cxcl12-expressing clusters in E18.5 FBM stroma revealed two fibroblast clusters (F-1 and F-1, both of which contained adipolineage gene signatures) and one pericyte cluster (P-2, expressing smooth muscle and myofibroblast-specific transcripts) (Figure 3-35C). These clusters were also all enriched for Kitl expression among E18.5 clusters (Figure 3-36C). Finally, E16.5 FBM stroma clusters enriched for Cxcl12 expression included four fibroblast clusters (F1 through F-4, all of which contained some cells with adipolineage gene signatures) and one undefined cluster (UD, which contained no clear expression of any lineage-specific 
A

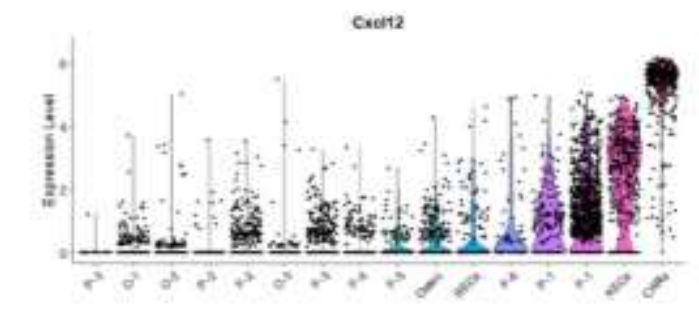

C

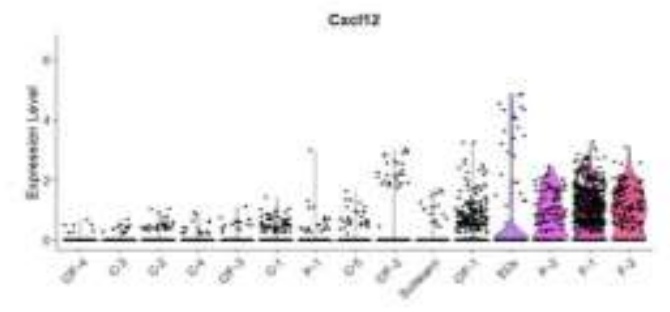

B PO

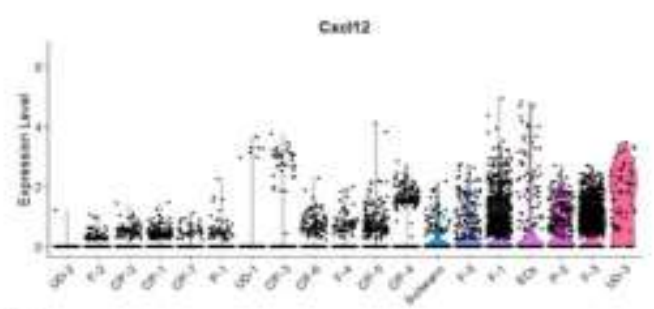

D $\quad$ E16.5

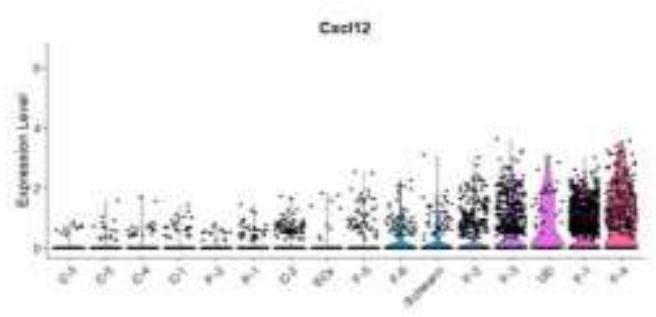

Figure 3-35. Stomal Cxcl12 expression across BM ontogeny

Violin plots depicting Cxcl12 expression within (A) adult, (B) P0, (C) E18.5, and (D) E16.5 single cell stromal clusters. CARs, CXCL12-abundant reticular cells; C, chondrocytes; C/F, chondrocyte/fibroblast; ECs, endothelial cells; AECs, arterial endothelial cells; SECs, sinusoidal endothelial cells; F, fibroblasts; Osteo, osteolineage; $P$, pericytes; Schwann, schwann cells; UD, undefined cluster. 
A

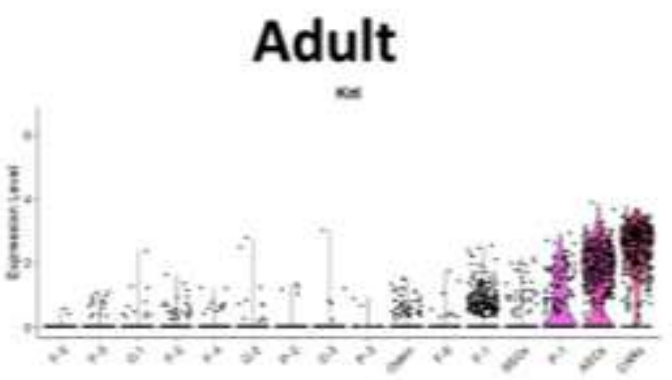

C

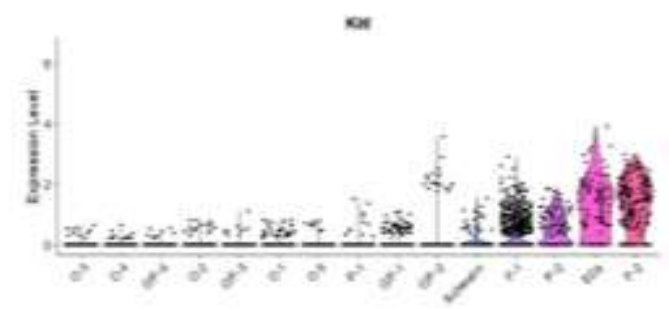

B PO

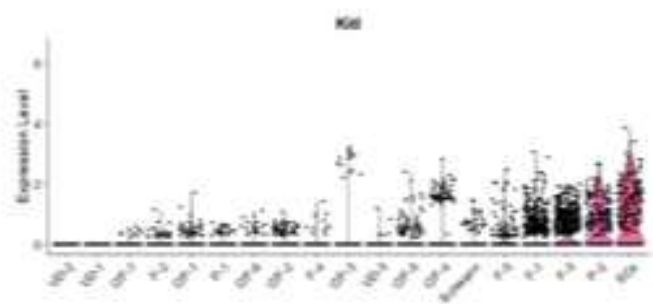

D

E16.5

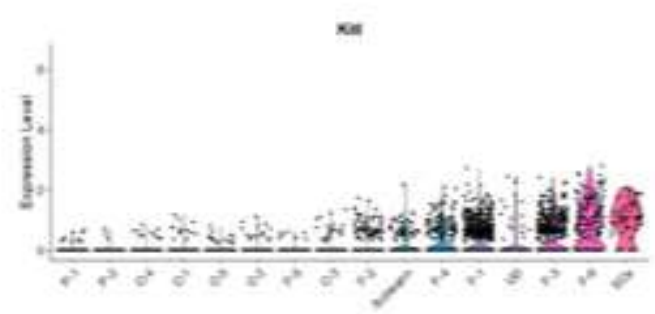

Figure 3-36. Stromal Kitl expression across BM ontogeny

Violin plots depicting Kitl expression within (A) adult, (B) P0, (C) E18.5, and (D) E16.5 single cell stromal clusters. CARs, CXCL12-abundant reticular cells; C, chondrocytes; $\mathrm{C} / \mathrm{F}$, chondrocyte/fibroblast; ECs, endothelial cells; AECs, arterial endothelial cells; SECs, sinusoidal endothelial cells; F, fibroblasts; Osteo, osteolineage; P, pericytes; Schwann, schwann cells; UD, undefined cluster. 
genes) (Figure 3-35D). All of these clusters with the exception of F-2 showed mid to low expression of Kitl, but were still relatively enriched for Kitl expression compared to other E16.5 FBM clusters (Figure 3-36D).

Together, our scRNA-Seq analysis of BM stromal populations presents a model in which the fetal and neonatal BM contains low levels of definable osteoblast, CAR cells, and endothelial cell subsets, but do contain putative niche cells for HSC maintenance. These putative niches appear to primarily consist of cells with fibroblast and, surprisingly, adipolineage gene signatures. Interestingly, an "adipo-CAR" subpopulation of CAR cells has been recently identified (Baccin 2019). 


\section{CHAPTER 4. DISCUSSION}

Our study represents a thorough dissection of the FBM hematopoietic compartment, and we are the first to report the presence of bona fide HSCs within the E15.5 FBM (Figure 4-1). Interestingly, the lineage output of these early FBM HSCs suggested that lineage-biased HSCs exist in the FBM space as early as E15.5. Due to our assessment of the BM compartment from the entire murine fetal skeleton compared to previous studies only using long bones (Christensen 2004, Coşkun 2014), we assumed that HSCs were present in other sources of BM. However, upon transplantation of BM from different sources, we found that HSCs were present in the forelimbs, hindlimbs, and trunk of E15.5 embryos (Figure 4-1). We are also the first to assess the BM immunophenotypic HSPC compartment from initial seeding to adulthood and found that MPP2s are the predominant HSPC population in the FBM and appear to have the ability to migrate to and seed the FBM directly from the FL (Figure 4-1).

However, immunophenotypic MPP2s are not functional in vitro or in vivo until birth (E18.5-P0), and display reduced repopulating capacity compared to adult BM and time-matched FL MPP2s - the same timepoint in which there is a shift in the frequencies of HSPCs in the BM to the more MPP3/MPP4-dominant phenotype seen in adult BM (Figure 4-1). This suggests the presence of intrinsic or extrinsic mechanisms controlling MPP2 functional maturation. To identify these mechanisms, we isolated stromal and HP populations from E16.5, E18.5, P0, and adult BM, and constructed the first known scRNA-Seq dataset spanning the HP and stromal compartment across BM ontogeny (Figure 3-28). Our preliminary studies have shown that the FBM stroma and HP compartments are distinct from the adult BM compartments, and this disparity in cell composition is even more drastic at E16.5 (Figures 3-29 and 3-34). For instance, the E16.5 FBM HP compartment is composed primarily of cells with T-cell lineage and mast cell lineage gene signatures, and immunophenotypic MPP2s and LT-HSCs show a marked reduction in stem cell associated transcriptional programs (Figures 3-29, 3-32, and 3-33). Moreover, no definable CAR cell gene signatures can be attributed to cell clusters from E16.5-P0 stromal libraries, and the primary cell clusters expressing factors important for HSPC maintenance in the BM niche are various fibroblast and pericyte clusters (Figures 3-34, 3-35, and 3-36). This suggests that the reduced function of FBM MPP2s - as well as the reduced expression of stem cell signatures in MPP2 and LTHSCs in the FBM—may be due to a semi-incompatible FBM niche.

In this section, I will discuss the implications and potential limitations of this study, as well as outline future experiments to answer more questions generated from our results.

\section{The Presence of Bona Fide HSCs in the E15.5 FBM}

Our study indicates the presence of bona fide HSCs in the E15.5 FBM (Figure 3-15), which has never been demonstrated. Previously, the earliest timepoint at which 


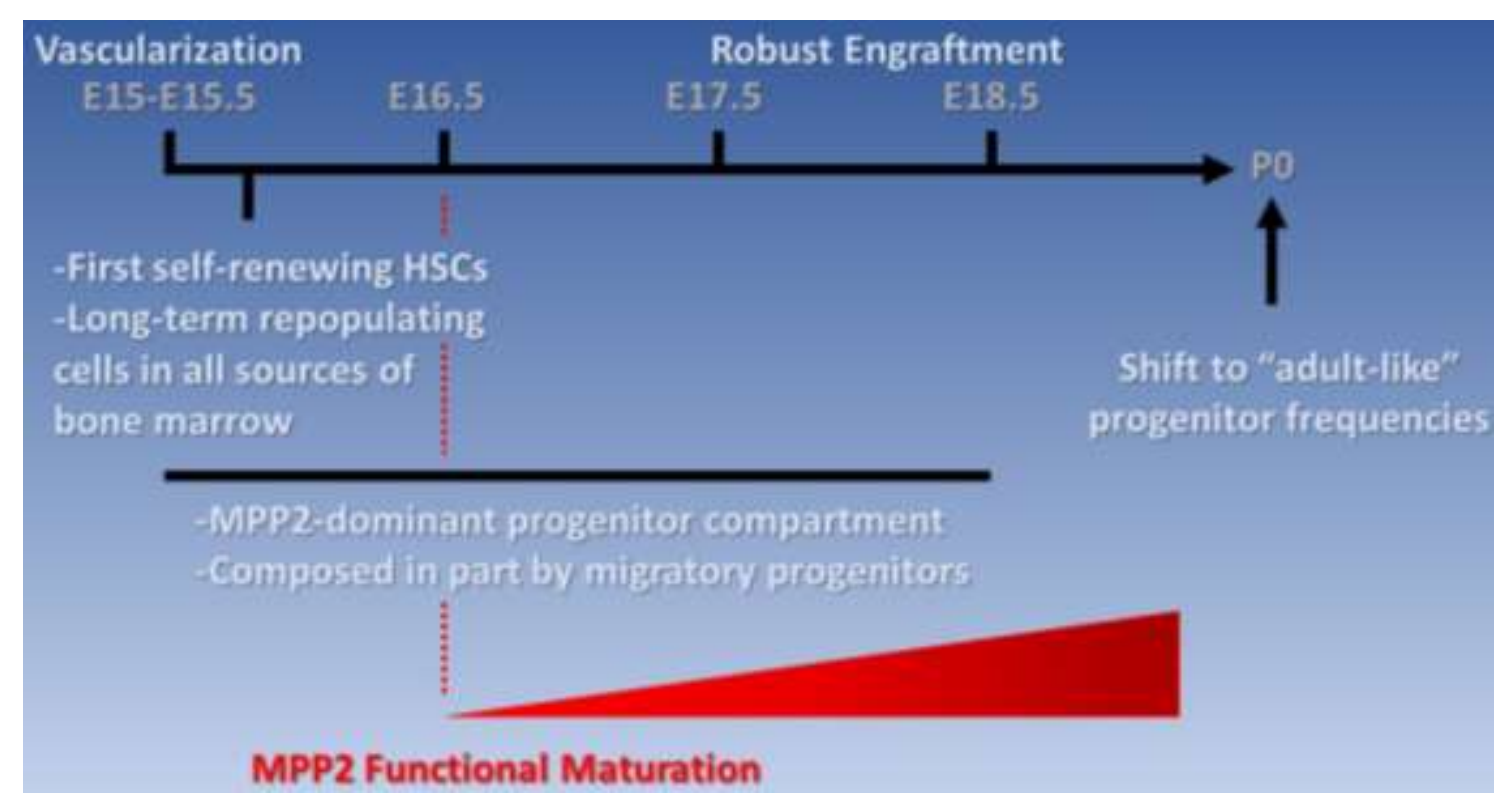

Figure 4-1. A revised developmental timeline of FBM hematopoiesis 
HSCs could be found in the FBM was E16.5 (Coşkun 2014). Why were we able to identify HSCs one day earlier? We initially thought that this was due to our approach to isolate the entire embryonic skeleton, but we were able to detect HSC activity in all bones analyzed, suggesting that the BM source is not a limiting factor in finding transplantable HSCs at E15.5 (Figure 3-17). Therefore, the observed HSC activity in E15.5 FBM could be due to various technical caveats.

First, the process of dissecting fetal skeletons and removing any contaminating tissues is not straightforward, and it is almost impossible to exclude all contaminating muscle or connective tissue. There is a possibility that some HSCs reside in the muscle or connective tissue of E15.5 embryos and are contaminating our BM preparations. Indeed, McKinney-Freeman et al. demonstrated that muscle HSCs (msHSCs) with long-term multilineage repopulating potential can be found in the muscle tissue of 6-8 week old mice, and they speculated that these msHSCs may be quiescent "developmental leftovers" from early in development (McKinney-Freeman 2002). However, the highest levels of long-term donor contribution were still relatively low (23\%) after transplanting $246,000 \mathrm{CD}^{+} 5^{+}$cells, and the CD45- cell fraction contained no HSCs (McKinneyFreeman 2002). In addition, the isolation of cell suspensions from muscle tissue required digestion with collagenase and trypsin followed by centrifugation in Percoll, while we simply crushed fetal bones followed by filtration (McKinney-Freeman 2002). This suggests that HSCs from muscle tissue are not contaminating our FBM preparations, although isolating E15.5 muscle and connective tissue followed by transplantation would unequivocally prove the lack of contaminating msHSCs.

Another source of contamination could be circulating blood at E15.5. Given that the mouse skeletons we use undergo several washes with PBS prior to BM isolation, it is unlikely that HSCs circulating in the PB are contaminants. It is important to note that a study identified the peak of HSC activity in the fetal PB at E14.5, followed by a drop at E15.5 (Christensen 2004). However, only 6/10 recipients displayed any donor contribution from E15.5 PB after transplantation of an entire embryo equivalent (e.e.) of blood into each recipient, while $1 / 7$ recipients were reconstituted with 0.1 e.e. of $\mathrm{PB}$ (Christensen 2004). Given that a substantial amount of PB is needed to provide donor reconstitution at E15.5 and that our method for skeleton BM preparations includes several washes of dissected skeletons, we suggest that HSCs from the PB are not a source of contamination in our E15.5 FBM preps.

A final source of technical error may come from variation in the true developmental age of E15.5 embryos. To secure embryos at desired developmental stages we employed timed pregnancies, in which we placed a female with a male overnight (after 5pm) and checked for the presence of a vaginal plug in the morning (before 10am). If a vaginal plug was detected, it was presumed that the female was pregnant, and the embryos were designated "E0.5." Obviously, this allows for several hours of variation in the true developmental age of the embryo. However, given that we injected FBM suspensions from 18 E15.5 embryos from multiple litters (Figure 3-7), we conclude that error in developmental staging was likely not to blame for our observations. In future experiments, it would be beneficial to employ the identification of specific morphological 
characteristics of E15.5 embryos when deciding which embryos to use for transplantation.

An understanding of BM development supports the possibility of HSCs residing in the BM at E15.5. The fetal bones of the limbs and trunk are formed from mesodermderived mesenchymal cells, which accumulate in the bone space at around E11 and form clusters in a process called "mesenchymal condensation" (Hall 2000, Olsen 2000)

(Figure 4-2A). Centrally located mesenchymal cells begin differentiating into chondrocytes that will proliferate and form a cartilage plate for later bone formation, while peripherally located mesenchymal cells differentiate into perichondrial cells (Matsushita 2020) (Figure 4-2B). At around E13-E14, the cartilage plate begins to expand through successive differentiation of hypertrophic chondrocytes, followed by the invasion of the central portion of the plate by perichondrial cells that subsequently differentiate into osteoblasts forming the bone collar (Kobayashi 2014b) (Figure 4-2C). Finally, around E15-E16 the central portion of the cartilage plate becomes vascularized, the BM space is formed, and cartilage begins to be replaced by osteoblast-derived bone (Kobayashi 2014b) (Figure 4-2D). E15.5 FBM HSC activity coincides with a recent report showing definitive vessel formation within the bone cavity at $\sim$ E15 (Langen 2017). However, this same report provides confocal images clearly depicting $\mathrm{Emcn}^{+}$vasculature adjacent to the bone cavity even earlier (E14.5), suggesting that the vasculature is primed to receive the necessary cues to begin vasularization of the bone (Langen 2017). It is possible that the first detectable BM-associated HSCs arrive at the fetal bone from the FL before E15.5 and are "lying in wait" in the vasculature adjacent to the bone until they can enter the bone cavity. Indeed, nearly two decades ago Kumaravelu et al. showed that E12-E13 forelimbs appeared to contain 0.5-1 HSCs capable of reconstitution posttransplant (Kumaravelu 2002). Therefore, there may even be cues in the mesenchymal condensate or cartilage plate before the formation of BM that facilitate the migration of HSCs to sites of future bone formation, and it would be interesting to determine if these theoretical HSCs migrate from the FL or directly from the AGM.

\section{Potential Mechanisms for the Shift in HSPC Frequencies Around Birth}

One of the most interesting revelations from our study was the shift in FBM HSPC frequencies right around birth from an MPP2-dominant phenotype to an MPP3/4dominant phenotype (Figure 3-19). While the early MPP2-dominant phenotype may only represent a subset of MPP2s considering their lack of functional ability until at least E18.5 (Figures 3-24 and 3-26), the shift at birth is still an interesting observation for follow up studies and we speculate that it could be due to a number of birth-related factors (Figure 4-3).

One potential contributor to the shift in HSPC frequencies around birth could be acute exposure to ambient oxygen upon delivery of newborn pups (Figure 4-3A). Indeed, by collecting cells from BM and cord blood in hypoxic $\left(3 \% \mathrm{O}_{2}\right)$ vs ambient $\left(21 \% \mathrm{O}_{2}\right)$ conditions, Mantel et al. found that ambient air collection decreased the number of LTHSCs at the expense of ST-HSCs and MPPs, suggesting that LT-HSCs initiate rapid 


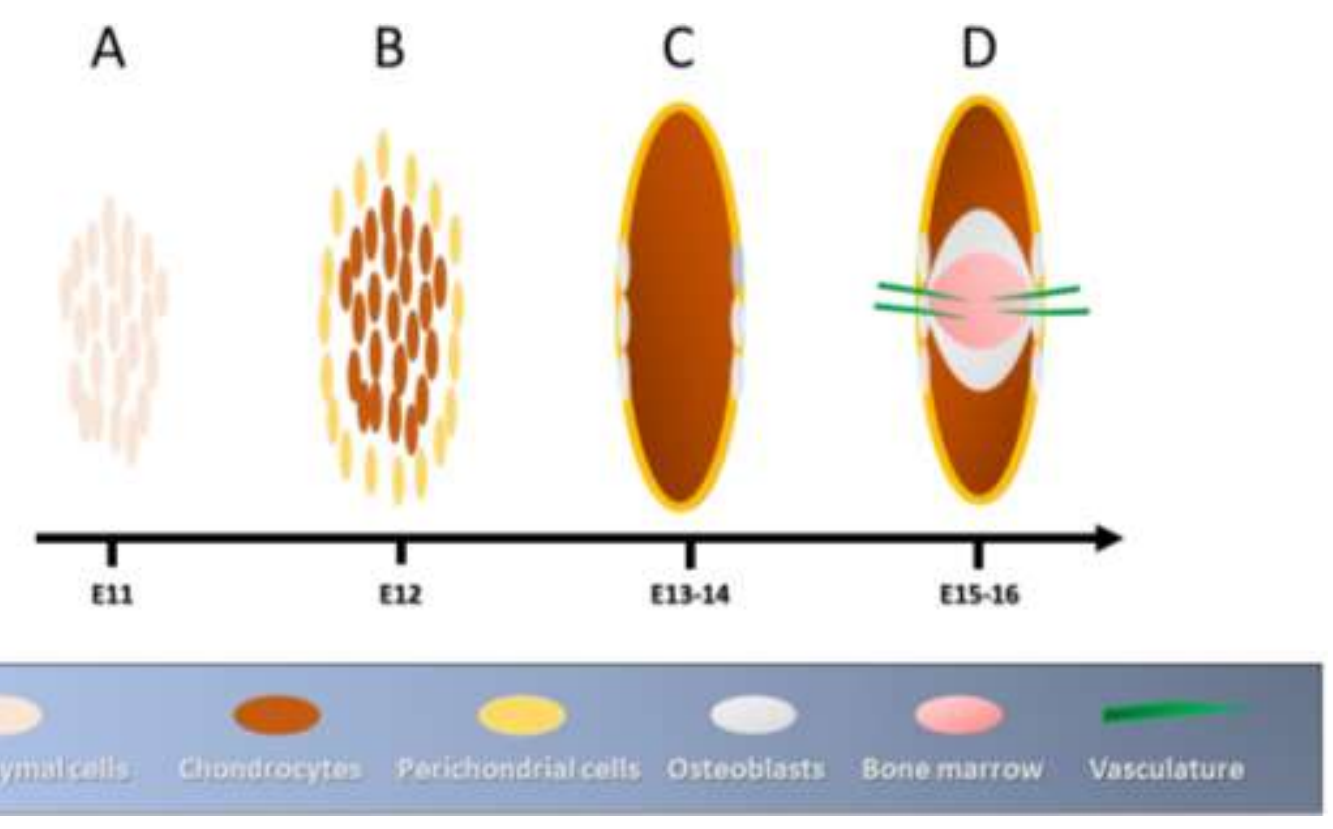

Figure 4-2. Development of the BM space

A. Mesoderm-derived mesenchymal cells form clusters in a process called "mesenchymal condensation." B. Central mesenchyme begins differentiating into chondrocytes ( the cartilage plate), while peripheral chondrocytes differentiate into perichondrial cells. C. The cartilage plate expands and perichondrial cells begin to differentitate into osteoblasts to form the bone collar. D. Vascularization of the central cartilage plate forms the BM space, and chondrocytes begin to be replaced by osteoblast-derived bone. 
A

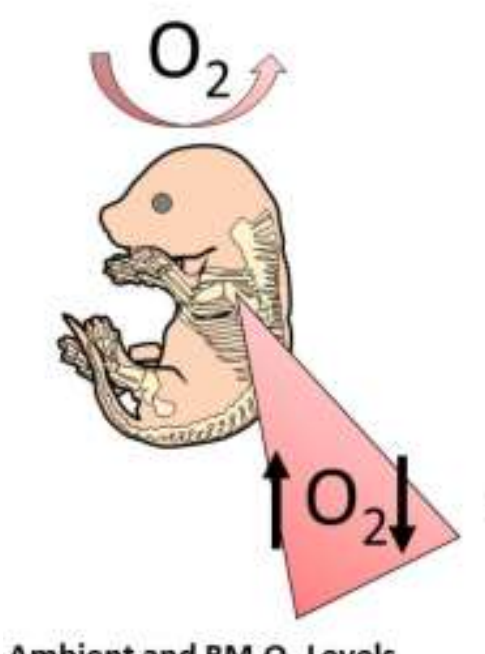

B

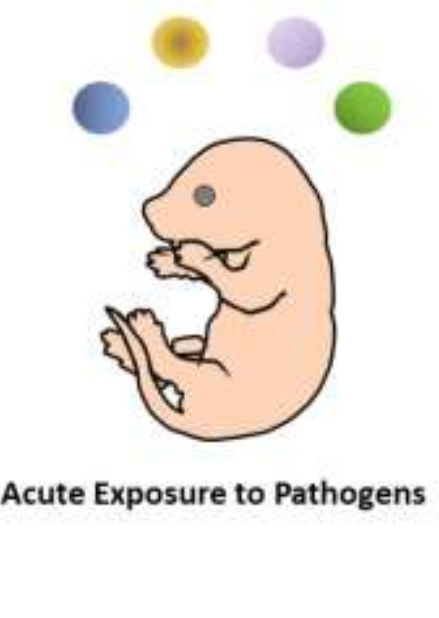

C

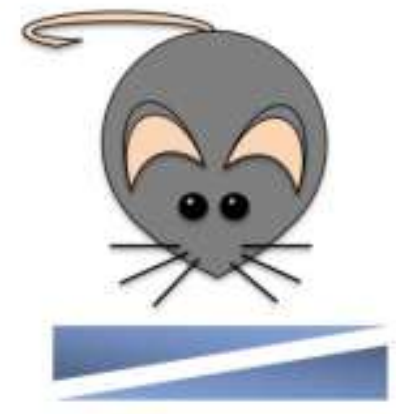

Intrauterine hormonal changes

Ambient and $\mathrm{BM} \mathrm{O}_{2}$ Levels

Figure 4-3. Potential effectors of HSPC frequency at birth

Putative explanations for HSPC immunophenotypic shift at birth, including (A) acute ambient oxygen exposure and/or changes in BM niche oxygen concentrations, (B) acute exposure to pathogens, or $(\mathrm{C})$ hormonal changes in the uterus during parturition. 
differentiation in response to high $\mathrm{O}_{2}$ concentrations (Mantel 2015). However, we see a marked increase in the total numbers of LT-HSCs and other HSPCs after birth, suggesting there is substantial differentiation and self-renewal—as well as continual migration of HSPCs from the FL-within the LT-HSC compartment during this time. It is important to note that the normal adult $\mathrm{BM}$ microenvironment in which HSCs reside is thought to be highly hypoxic (Morrison 2014), and the oxygen levels of the HSC/HSPC niche in the fetal and newborn BM have yet to be assessed and may undergo significant changes as the cellular composition of the niche changes during development. For instance, the findings of Mantel et al. may support our results if the E16.5-E18.5 FBM displays oxygen levels closer to ambient air, but then becomes hypoxic during or after birth. In future studies it will be important to assess $\mathrm{O}_{2}$ levels in the FBM niche to see if this is a contributing factor to HSPC frequency dynamics during birth.

Upon delivery the neonate transitions from a theoretically sterile environment in the uterus to an environment filled with microbes and pathogens and can even be colonized by microbes while in the maternal vaginal canal (Dominguez-Bello 2010). Many groups have demonstrated that the gut microbiome of adult mice is critical for normal steady-state hematopoiesis, including the production of several myeloid cells in the BM (Balmer 2014). In one of the few studies analyzing the effects of dysregulated gut microbiome on BM HSPCs, Josefsdottir et al. showed that germ-free or antibiotic-treated mice have fewer numbers of LT-HSCs and downstream progenitors, and this is likely due to increased quiescence within the LT-/ST-HSC compartments (Josefsdottir 2017). This suggests that various bacterial metabolites or their action on other cellular components of the BM niche may be important for HSPC proliferation. These metabolites may also be contributing factors to the burst of HSPCs we see in the FBM around birth. We plan to test this hypothesis in future studies by analyzing the fetal and newborn BM compartments in germ-free mice or in fetuses/neonates bore from antibiotic-treated mothers (Figure 4-3B).

Finally, hormonal changes within the uterine environment may play a role in fetal hematopoiesis (Figure 4-3C). During parturition, the uterus undergoes several changes to facilitate proper delivery. The initiation of labor in mice can be identified by a reduction in progesterone levels in the uterus, which are required for normal uterine quiescence (Sugimoto 1997). This drop in progesterone is concomitant with a breakdown of the uterine wall, myometrial contractions, and cervical ripening, all of which are mediated by the production or degradation of a myriad of hormones (Ratajczak 2008). Prostaglandins [primarily prostaglandin $\mathrm{F}_{2 \alpha}\left(\mathrm{PGF}_{2 \alpha}\right)$ and prostaglandin $\left.\mathrm{E}_{2}\left(\mathrm{PGE}_{2}\right)\right]$ are highly upregulated in the uterus around birth and are thought to have a key role in these processes, as $\mathrm{PGF}_{2 \alpha^{-}}$ ${ }^{1-}$ mice do not initiate parturition (Sugimoto 1997). Interestingly, exposure of murine BM to $\mathrm{PGE}_{2}$ appears to facilitate HSPC expansion, survival, and homing ability (North 2007, Porter 2013), and many fetal tissues — including the placenta - express several prostaglandin receptors. It is therefore enticing to imagine uterine hormones somehow affecting fetal hematopoietic programs. The mechanisms involved in labor initiation are well-studied due to the prevalence of premature births (Ratajczak 2008), and we are currently assessing the literature for potential HSPC regulators. 


\section{Migration of HPSCs to the FBM}

The accepted model of HSC accumulation in the BM suggests that HSCs migrate from the FL in a CXCL12/CXCR4-dependent manner, seed the BM, and likely expand from E18.5 to P21 (Ara 2003, Bowie 2006, Bowie 2007, Medvinsky 2011). To our knowledge, our study is the first to show that several HSPC subsets from the E15.5-P0 liver can migrate toward a CXCL12 gradient, suggesting that the FBM is seeded by several progenitors, not solely HSCs (Figure 3-22). However, this does not explicitly prove that all HSPCs migrate and seed the FBM at E15.5, and we can think of three models for HSPC seeding of the FBM (Figure 4-4).

The first model (Figure 4-4A) suggests that only LT-HSCs migrate from the FL to the FBM at E15.5, followed by a propensity for rapid MPP2-biased differentiation. This model can be supported by what is known about the dynamics of LT-HSC differentiation into the various HSPC subsets, as MPP2s have been shown to be the first HSPC subset produced by LT-HSCs in in vitro differentiation assays (Pietras 2015). MPP2s also appear to share some of the same transcriptional programs as LT-HSCs, as $\mathrm{Mpl}^{-/-}$mice display defects in LT-/ST-HSC and MPP2 production, while no other HSPC subset appears to be affected (Pietras 2015). Finally, Pietras et al. also found that myeloid-biased HSPCs (MPP2/MPP3) were rapidly expanded shortly after transplantation of LT-HSCs, suggesting that LT-HSCs preferentially reconstitute the myeloid compartment of irradiated recipients after transplantation (Pietras 2015). The notion that LT-HSCs are indeed the primary migratory cells from the FL to the FBM and that the expansion of MPP2s in the E15.5 FBM is a result of the preferential differentiation of LT-HSCs toward a myeloid-biased HSPC is tempting to consider, because it would suggest that the behavior of LT-HSCs in adult BM transplantation is a mimicry of the inherent behavior they exhibit during ontogeny while seeding the FBM.

The second model (Figure 4-4B) proposes that all HSPCs can migrate to the FBM at E15.5, and this model is supported by our in vitro migration assays (Figure 3-22). However, what is not accounted for in our study is the enhanced presence of MPP2s. There are many reasons migratory MPP2s could be the dominant compartment in the FBM. It could be that FL MPP2s can migrate toward a greater number or more specific FBM-derived signals compared to other HSPCs. Indeed, Christensen et al. found that FL LT-HSCs migrate in response to both CXCL12 and SCF gradients, while adult LT-HSCs appear to preferentially migrate toward CXCL12 (Christensen 2004). FL MPP2s may also express higher levels of adhesion molecules (cadherins, selectins, integrins) or matrix metalloproteinases for more efficient retention or invasion of the FBM niche, respectively (Ciriza 2013). FL MPP2s may also have greater CXCL12 sensitivity, which could be assessed by exposing FL HSPCs to various CXCL12 concentration gradients. However, given that FBM MPP2s appear to have little functional ability post-transplant and adult MPP2s are also a transient population after transplantation (Pietras 2015), it is unlikely that migratory FL MPP2s undergo rapid selfrenewal and expansion upon FBM entry.

The final model (Figure 4-4C) is a combination of the first and second models, 


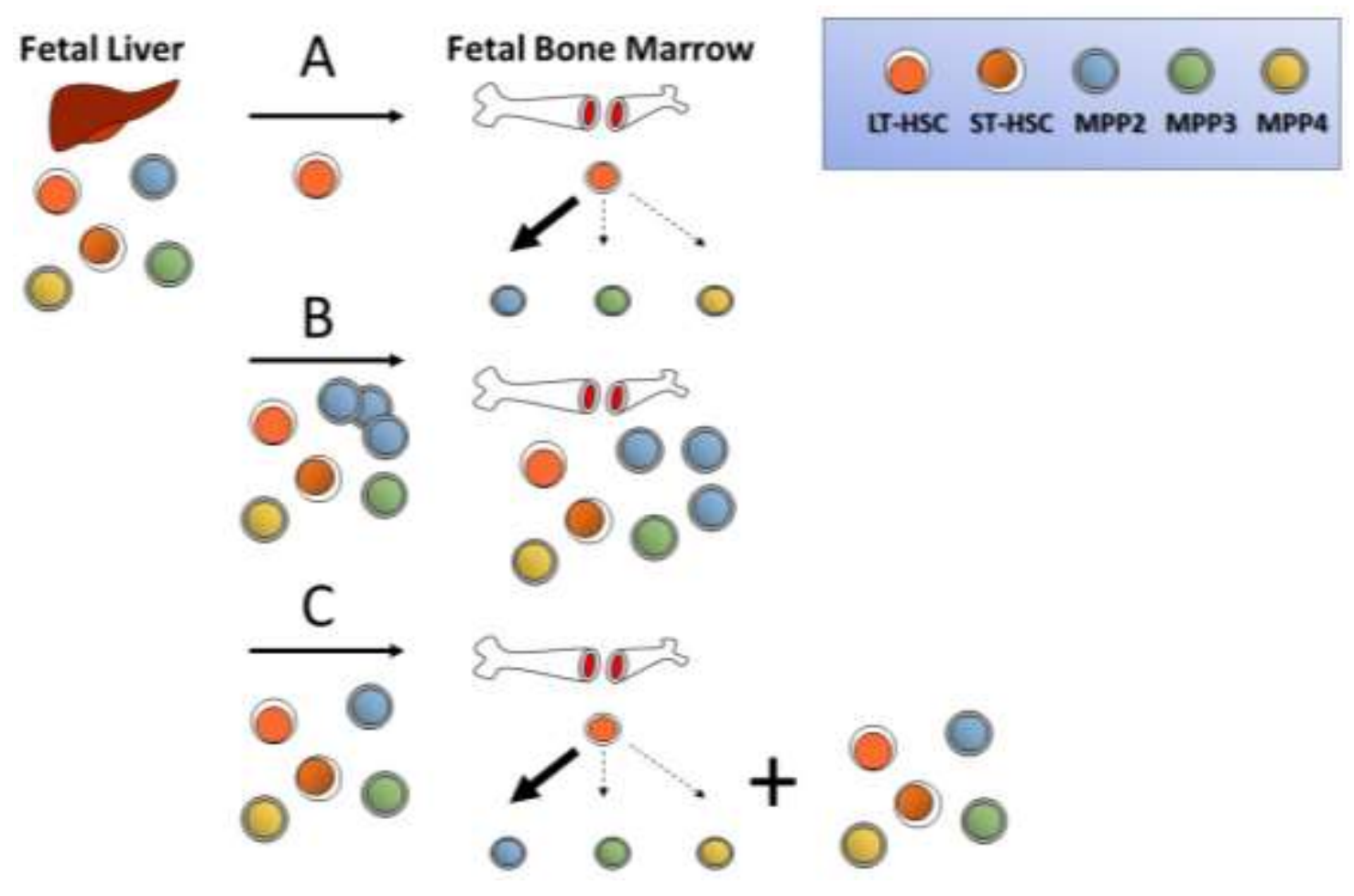

Figure 4-4. Models for HSPC migration and expansion in the FBM

Three models for HSPC migration and expansion in the fetal bone marrow: A. LT-HSCs migrate independently of other HSPCs and expand the HSPC pool in the FBM, with a bias toward MPP2 expansion. B. All HSPCs migrate from the FL to the FBM, with MPP2s possibly displaying an enhanced migratory potential. C. A mixture of the first two models, in which all HSPCs migrate from the FL to the FBM, but LT-HSCs also preferentially produce MPP2s. 
suggesting that all FL HSPCs migrate into the FBM while LT-HSCs preferentially differentiate into MPP2s. Given the results of our migration assays, the preponderance of MPP2s at early FBM timepoints, and the preference for LT-HSCs to produce myeloidbiased HSPCs (Pietras 2015), we conclude that this is currently the best available model. This model also raises more questions about the heterogeneity within HSPC subsets, as it would suggest the presence of migratory FL HSPCs and FBM LT-HSC-derived HSPCs coexisting in the FBM space.

\section{Functional Heterogeneity and Maturation of BM HSPCs}

Upon noticing that the frequency of MPP $2 s$ in the CD45 compartment of the early FBM was relatively high compared to later timepoints and that there was a lag in $\mathrm{CD}_{4} 5^{+}$blood production until around birth (Figure 3-23), we hypothesized that FBM MPP2s may display reduced functional potential until late in gestation. Our hypothesis was supported, as FBM MPP2s did not display robust in vitro or in vivo functional potential until P0 (Figures 3-24 and 3-26). Determining the reasons for this discrepancy in functional output is still ongoing, but our preliminary scRNA-Seq analysis (Figure 3-28) has provided some clues.

Utilization of CITE-Seq antibodies to prospectively identify immunophenotypic HSPCs allowed us to locate MPP2s within annotated cell clusters in our scRNA-Seq libraries (Figures 3-30 and 3-31). Given the functional heterogeneity of the MPP2 compartment even within adult BM (Figures 3-24 and s3-26) (Pietras 2015), it was not surprising to find that adult immunophenotypic MPP2s are found within several distinct cell clusters, spanning myeloid progenitors to B-cell lineages (Figure 3-31). Therefore, it is more appropriate to think of the immunophenotypic MPP2 as a mixture of cells that contains a subset of myeloid-biased progenitors - at least by conventional differentiation assays. This cell cluster heterogeneity among immunophenotypic MPP $2 \mathrm{~s}$ was consistent from E16.5 to P0 (Figure 3-31). Since no cell surface molecules have been identified to enrich for different cell subsets within the MPP2 compartment, one possibility for the observed "maturation" of MPP2s from E16.5 to P0 may not be due to maturation at all, but simply an accumulation of the functional MPP2 subset through migration or differentiation from LT-HSCs (Figure 4-5A). We are currently analyzing the different subsets of immunophenotypic MPP2s for putative cell surface markers to enrich for functional MPP2s.

We were also able to incorporate the hscScore algorithm (Wilson 2015, Hamey 2019) into our scRNA-Seq dataset, which provides a value for the "stemness" of each single cell based on the expression of HSC-associated genes. Incorporation of hscScore to other scRNA-Seq datasets has shown a progressive decline in hscScore from LT-HSCs to committed progenitors, and MPP2s have been previously assessed for hscScore (Hamey 2019). After subclustering immunophenotypic MPP2s, we observed a progressive increase in hscScore from E16.5 to P0, and this was mainly due to the accumulation of a small subpopulation of hscScore ${ }^{\text {hi }}$ cells (Figure 3-32). This could represent an increase in the proposed functional subset of immunophenotypic MPP2s. 
A

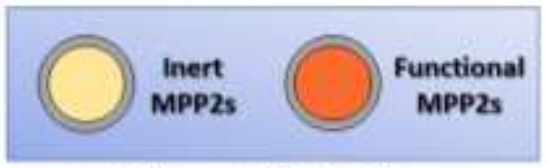

Fetal Liver Adult Bone Marrow

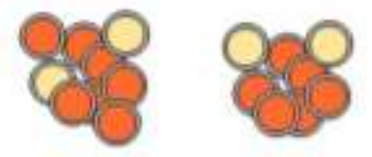

Fetal Bone Marrow

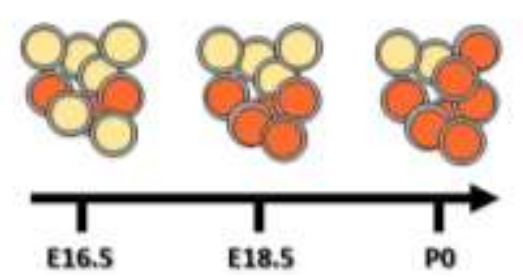

B

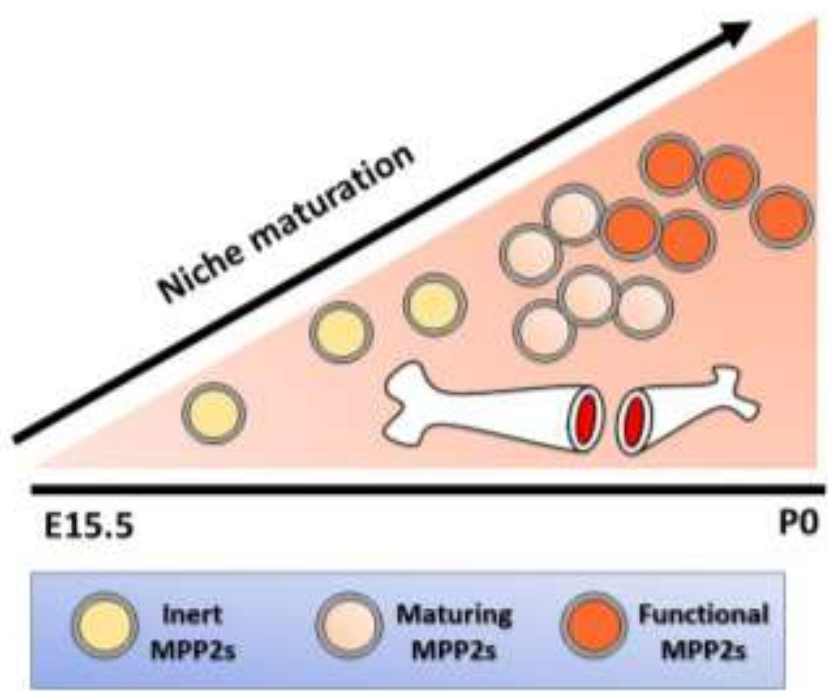

Figure 4-5. Hypotheses for FBM MPP2 functional maturation

A. Accumulation of a subset of functional MPP2s through migration from the FL or differentiation of LT-HSCs. In this scenario, there is not a true "maturation" step, but merely an expansion of a subset of immunophenotypic MPP $2 \mathrm{~s}$ which contain intrinsic functional competency. B. Niche maturation contributing to the maturation of the MPP2 compartment. In this scenario, the development of the FBM niche results in the prevalence of factors important for MPP2 functional maturation, indicating extrinsic control on the MPP2 compartment. 
Importantly, we observed the same increase in hscScore ${ }^{\text {hi }}$ cells from immunophenotypic LT-HSCs from E16.5 to P0, suggesting an accumulation of the functional LT-HSC subset across late gestation as well (Figure 3-33). Also, immunophenotypic LT-HSCs showed greater cell cluster heterogeneity at E16.5 compared to E18.5 and P0, supporting a model for enrichment of the functional LT-HSC subset around birth (Figure 3-31). Future experiments will be aimed at determining if immunophenotypic LT-HSCs - and other HSPC subsets - also display a trend toward increased functional competency around birth compared to earlier timepoints via in vitro and in vivo methods.

It is important to note that hscScore was developed using expression data on highly transplantable HSCs (Wilson 2015, Hamey 2019). Therefore, the observed correlation between MPP2 hscScore and functional maturation may simply be due to the expression of factors important for engraftment or homing during transplantation and may provide little information regarding the maturation of MPP2s important for homeostatic maintenance in the embryo. More studies are needed to fully dissect the heterogeneous MPP2 compartment during adulthood and development, as there may be a subpopulation of these cells that are important for proper blood production during steady state which cannot be assayed by conventional methods.

\section{Maturation of the FBM Niche}

Our scRNA-Seq data revealed a dramatic reconstruction in the cellular composition of the hematopoietic niche from E16.5 to P0, as well as of the stromal niche from perinatal timepoints compared to the adult (Figures 3-29 and 3-34). Given that niche interactions with HSPCs are vital to their maintenance and a number of aberrations in the niche can disrupt their function (Crane 2017, Gao 2018), we suspect that the reconstruction of the FBM niche during late gestation could be a source for the functional maturation of MPP2s and other HSPCs (Figure 4-5B).

In the adult BM, stromal cells appear to be the most well-studied components of the HSC niche, with a focus on the arterial and sinusoidal niches. Both sources of BM vasculature are thought to be in close association with Nestin $^{+}$perivascular cells, which express high levels of the HSC maintenance factors Cxcl12 and Kitl (Crane 2017, Gao 2018). These perivascular cells are colloquially called "CXCL12 abundant reticular cells," or CAR cells, and their ablation invariably results in HSC mobilization, reduction in quiescence, and overall reduced function. As expected, we were able to annotate a clear CAR cell population in our adult BM scRNA-Seq data, but the presence of Cxcl12 and/or Kitl expression in newborn or fetal BM libraries was severely reduced, and appeared to be equally distributed among several fibroblast and pericyte cell types (Figures 3-34, 3-35, and 3-36). Of note, Isern et al. identified rare Nestin ${ }^{+}$cells within the E17.5-E18.5 FBM that expressed low levels of Cxcl12, Kitl, and other HSC maintenance factors (Isern 2014). They also found that deletion of Nestin ${ }^{+}$cells in E15.5 mice resulted in a significant increase in the number of HSCs found in the E17.5 liver with a concomitant decrease in HSC numbers in the E17.5 FBM via an in vitro long-term culture initiating cell assay (Isern 2014), suggesting that Nestin ${ }^{+}$cells in the FBM are 
important for proper migration of HSCs to the FBM. However, they did not test HSCs or any other HSPC for in vivo functional potential at this timepoint. If these $\mathrm{Nestin}^{+}$cells are truly a niche for HSPCs in the FBM, it is possible that the low HSPC numbers and functional impairment of MPP2s is simply a reflection of a reduction in available niche space due to the rarity of $\mathrm{Nestin}^{+}$cells - as well as their reduced expression of critical niche factors. Indeed, it is still unclear if some other cell type is a contributor to the expansion and functional maturation of HSPCs during the perinatal period, as the authors note that FBM Nestin ${ }^{+}$cells appear to be mostly quiescent (Isern 2014). However, they only assess the total number of Nestin ${ }^{+}$cells from P0 to adulthood (Isern 2014).

Hematopoietic cells are also thought to contribute to the maintenance of HSPCs in the adult $\mathrm{BM}$ niche. As described earlier, megakaryocytes, macrophages, regulatory $\mathrm{T}$ cells, and others are all thought to control HSC function (Crane 2017, Gao 2018). Incredibly, we found that the hematopoietic progenitor (HP) compartment of the E16.5 FBM consisted of several mast cell and T-cell lineage clusters, comprising nearly $80 \%$ of all cells (Figure 3-29). It is generally thought that HSCs give rise to common lymphoid progenitors in the bone marrow, which then migrate to the thymus for T-cell maturation (Kondo 1997). Therefore, the T-cell lineage clusters in our E16.5 dataset likely represent some sort of T-cell primed lymphoid progenitor. Considering that they are present as early as E16.5, these progenitors may even be comprised of migratory cells from the FL to the FBM before seeding the fetal thymus, as pre-thymic T-cell progenitors have been identified in the FL as early as E12 (Watanabe 1997, Kawamoto 2000).

In the adult mouse, mast cell progenitors are thought to arise from an upstream myeloid progenitor in the BM, where they will then migrate to different tissues and undergo differentiation (Dahlin 2015b). Meanwhile, fetal mast cell progenitors have been found in E9-E11 YS, followed by their appearance in the FL (Sonoda 1983). Rodewald et al. identified a Thy $1{ }^{\mathrm{lo}} \mathrm{c}-\mathrm{Kit}^{+}$mast cell progenitor that appeared in the fetal blood at E14.5, peaked in prevalence at E15.5, then decreased until disappearance around birth (Rodewald 1996). Interestingly, he could not find a similar population in the FL during mid or late gestation, suggesting that it was either arising from a different source or underwent immunophenotypic changes upon entry into the fetal blood stream (Rodewald 1996). It is alluring to speculate whether this mast cell progenitor population is taking residence in the FBM around this time point and is the contributor to the several mast cell lineage cell clusters we have identified in our E16.5 FBM library. Alternatively, the abundant MPP2 population could be giving rise to these mast cell progenitors at early FBM timepoints and contributing to the pool that Rodewald observed in his studies.

T-cells and mast cells have been shown to play significant roles in innate immunity (St. John 2013, Seyda 2016), and the accumulation of their progenitors in the early FBM may be indicative of an effort to expand T-cell and mast cell lineages for the ensuing onslaught of pathogen exposure following birth. Therefore, the early FBM niche may be preferentially geared toward production of these lineages at the expense of functional niche space for HSPCs. It is also important to note that mast cell progenitors and their progeny express high levels of c-Kit on their cell surface. Given that we sorted for $\mathrm{c}-\mathrm{Kit}^{+}$cells for our scRNA-Seq analyses, it is possible that some of the mast cell 
lineage clusters in our E16.5 FBM library consist of mature mast cells. If this is the case, the E16.5 FBM may be a characterized by high levels of inflammation, which has been shown to affect HSPC mobilization, survival, proliferation, and myeloid-biased differentiation (Pietras 2017).

\section{Conclusions and Future Directions}

This study represents one of the only comprehensive assessments of hematopoiesis in the perinatal BM, revealing that bona fide HSCs colonize all sources of fetal bone by E15.5, birth appears to have a role in shifting HSPC frequencies, and specific HSPC populations undergo functional changes right around birth (Figure 4-1). We also provide the first scRNA-Seq data set for HP and stromal cells spanning the perinatal BM, which we hope will be utilized for future studies to elucidate specific mechanisms controlling hematopoietic and stromal cell processes during this critical window in development. Our study leaves several questions left to be answered concerning the FBM niche: Are there undiscovered subpopulations of MPP2s with disparate contributions to steady-state hematopoiesis? Do other HSPCs display similar functional maturation in the FBM during late gestation? Is there a specific regulatory niche that HSPCs reside in, and is it substantially rearranged around birth? Can maternal hormonal changes affect fetal hematopoiesis?

Our future studies will be focused on answering many of these questions. To identify intrinsic differences between immunophenotypic HSPC populations across FBM ontogeny, we are performing gene ontology analyses using our scRNA-Seq data (Figure 4-6A). This will allow us to understand the transcriptional programs specific to HSPCs at each timepoint. To define putative niches for HSPCs in the FBM, we are utilizing algorithms such as RNA-Magnet (Baccin 2019), which assesses BM-specific ligandreceptor expression profiles between cell populations (Figure 4-6B). Importantly, we will be comparing HP vs HP, HP vs stroma, and stroma vs stroma libraries to fully visualize the changing cellular interactome in the FBM. Our hope is that these analyses will identify novel hematopoietic factors in the FBM niche that can be therapeutically exploited to enhance HSC expansion/function/differentiation in the clinic. 
A

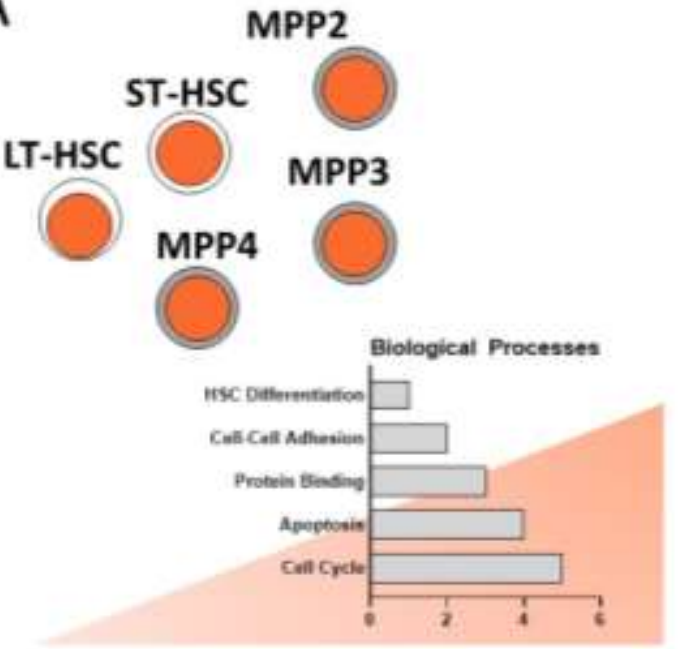

Unique progenitor signatures

across development?
B

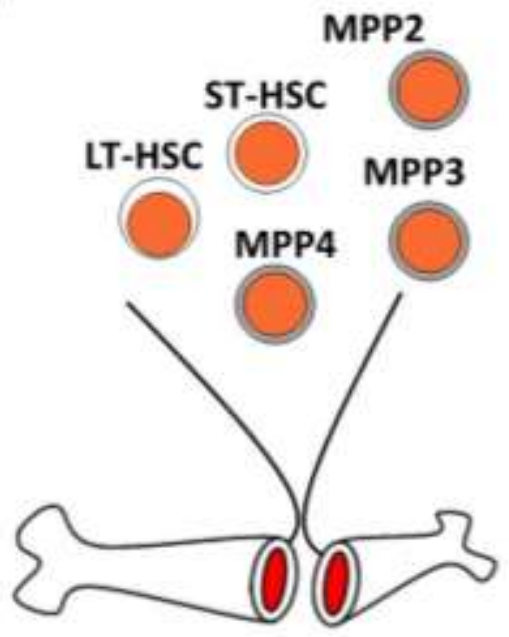

Putative niches for progenitors across development?

Figure 4-6. Ongoing scRNA-Seq analyses

Ongoing scRNA-Seq analyses, including (A) Gene Ontology analysis to identify changes in immunophenotypic progenitors across development and (B) RNA-Magnet analysis to identify putative stromal/hematopoietic niches for HSPCs during perinatal bone development. 


\section{LIST OF REFERENCES}

Adolfsson J, Borge OJ, Bryder D, Theilgaard-Mönch K, Åstrand-Grundström I, Sitnicka E, et al. Upregulation of Flt3 Expression within the Bone Marrow Lin-Sca1+c-kit+ Stem Cell Compartment Is Accompanied by Loss of Self-Renewal Capacity. Immunity. 2001;15(4):659-69. doi: 10.1016/s1074-7613(01)00220-5.

Adolfsson J, Mansson R, Buza-Vidas N, Hultquist A, Liuba K, Jensen CT, et al. Identification of Flt3 + lympho-myeloid stem cells lacking erythro-megakaryocytic potential a revised road map for adult blood lineage commitment. Cell. 2005;121(2):295306. Epub 2005/04/27. doi: 10.1016/j.cell.2005.02.013. PubMed PMID: 15851035.

Aihara Y, Buhring HJ, Aihara M, Klein J. An attempt to produce "pre-T" cell hybridomas and to identify their antigens. Eur J Immunol. 1986;16(11):1391-9. Epub 1986/11/01. doi: 10.1002/eji.1830161113. PubMed PMID: 3096743.

Akashi K, Traver D, Miyamoto T, Weissman IL. A clonogenic common myeloid progenitor that gives rise to all myeloid lineages. Nature. 2000;404(6774):193-7. Epub 2000/03/21. doi: 10.1038/35004599. PubMed PMID: 10724173.

Alvarez-Silva M, Belo-Diabangouaya P, Salaun J, Dieterlen-Lievre F. Mouse placenta is a major hematopoietic organ. Development. 2003;130(22):5437-44. Epub 2003/09/26. doi: 10.1242/dev.00755. PubMed PMID: 14507780.

Ara T, Tokoyoda K, Sugiyama T, Egawa T, Kawabata K, Nagasawa T. Long-Term Hematopoietic Stem Cells Require Stromal Cell-Derived Factor-1 for Colonizing Bone Marrow during Ontogeny. Immunity. 2003;19(2):257-67. doi: 10.1016/s10747613(03)00201-2.

Asada N, Kunisaki Y, Pierce H, Wang Z, Fernandez NF, Birbrair A, et al. Differential cytokine contributions of perivascular haematopoietic stem cell niches. Nat Cell Biol. 2017;19(3):214-23. Epub 2017/02/22. doi: 10.1038/ncb3475. PubMed PMID: 28218906; PubMed Central PMCID: PMCPMC5467892.

Baccin C, Al-Sabah J, Velten L, Helbling PM, Grunschlager F, Hernandez-Malmierca P, et al. Combined single-cell and spatial transcriptomics reveal the molecular, cellular and spatial bone marrow niche organization. Nat Cell Biol. 2020;22(1):38-48. Epub 2019/12/25. doi: 10.1038/s41556-019-0439-6. PubMed PMID: 31871321.

Balazs AB, Fabian AJ, Esmon CT, Mulligan RC. Endothelial protein C receptor (CD201) explicitly identifies hematopoietic stem cells in murine bone marrow. Blood. 2006;107(6):2317-21. Epub 2005/11/24. doi: 10.1182/blood-2005-06-2249. PubMed PMID: 16304059; PubMed Central PMCID: PMCPMC1895725. 
Balmer ML, Schurch CM, Saito Y, Geuking MB, Li H, Cuenca M, et al. Microbiotaderived compounds drive steady-state granulopoiesis via MyD88/TICAM signaling. J Immunol. 2014;193(10):5273-83. Epub 2014/10/12. doi: 10.4049/jimmunol.1400762. PubMed PMID: 25305320.

Baryawno N, Przybylski D, Kowalczyk MS, Kfoury Y, Severe N, Gustafsson K, et al. A Cellular Taxonomy of the Bone Marrow Stroma in Homeostasis and Leukemia. Cell. 2019;177(7):1915-32 e16. Epub 2019/05/28. doi: 10.1016/j.cell.2019.04.040. PubMed PMID: 31130381 ; PubMed Central PMCID: PMCPMC6570562.

Baum CM, Weissman IL, Tsukamoto AS, Buckle AM, Peault B. Isolation of a candidate human hematopoietic stem-cell population. Proc Natl Acad Sci U S A. 1992;89(7):28048. Epub 1992/04/01. doi: 10.1073/pnas.89.7.2804. PubMed PMID: 1372992; PubMed Central PMCID: PMCPMC48751.

Becker AJ, McCulloch EA, Till JE. Cytological demonstration of the clonal nature of spleen colonies derived from transplanted mouse marrow cells. Nature. 1963;197:452-4. Epub 1963/02/02. doi: 10.1038/197452a0. PubMed PMID: 13970094.

Becker AJ, McCulloch EA, Siminovitch L, Till JE. The Effect of Differing Demands for Blood Cell Production on DNA Synthesis by Hemopoietic Colony-Forming Cells of Mice. Blood. 1965;26(3):296-308. doi: 10.1182/blood.V26.3.296.296.

Benveniste P, Frelin C, Janmohamed S, Barbara M, Herrington R, Hyam D, et al. Intermediate-term hematopoietic stem cells with extended but time-limited reconstitution potential. Cell Stem Cell. 2010;6(1):48-58. Epub 2010/01/16. doi:

10.1016/j.stem.2009.11.014. PubMed PMID: 20074534.

Benz C, Copley MR, Kent DG, Wohrer S, Cortes A, Aghaeepour N, et al. Hematopoietic stem cell subtypes expand differentially during development and display distinct lymphopoietic programs. Cell Stem Cell. 2012;10(3):273-83. Epub 2012/03/06. doi: 10.1016/j.stem.2012.02.007. PubMed PMID: 22385655.

Bertrand JY, Jalil A, Klaine M, Jung S, Cumano A, Godin I. Three pathways to mature macrophages in the early mouse yolk sac. Blood. 2005a;106(9):3004-11. Epub 2005/07/16. doi: 10.1182/blood-2005-02-0461. PubMed PMID: 16020514.

Bertrand JY, Giroux S, Golub R, Klaine M, Jalil A, Boucontet L, et al. Characterization of purified intraembryonic hematopoietic stem cells as a tool to define their site of origin. Proc Natl Acad Sci U S A. 2005b;102(1):134-9. Epub 2004/12/30. doi: 10.1073/pnas.0402270102. PubMed PMID: 15623562; PubMed Central PMCID: PMCPMC544043.

Bertrand JY, Chi NC, Santoso B, Teng S, Stainier DY, Traver D. Haematopoietic stem cells derive directly from aortic endothelium during development. Nature. 
2010;464(7285):108-11. Epub 2010/02/16. doi: 10.1038/nature08738. PubMed PMID: 20154733; PubMed Central PMCID: PMCPMC2858358.

Bertrand JY, Desanti GE, Lo-Man R, Leclerc C, Cumano A, Golub R. Fetal spleen stroma drives macrophage commitment. Development. 2006;133(18):3619-28. Epub 2006/08/18. doi: 10.1242/dev.02510. PubMed PMID: 16914502.

Billingham RE, Lampkin GH, Medawar PB, Williams HL. Tolerance to homografts, twin diagnosis, and the freemartin condition in cattle. Heredity. 1952;6(2):201-12. doi: 10.1038/hdy.1952.20.

Billingham RE, Brent L, Medawar PB. Actively acquired tolerance of foreign cells. Nature. 1953;172(4379):603-6. Epub 1953/10/03. doi: 10.1038/172603a0. PubMed PMID: 13099277.

Boiers C, Carrelha J, Lutteropp M, Luc S, Green JC, Azzoni E, et al. Lymphomyeloid contribution of an immune-restricted progenitor emerging prior to definitive hematopoietic stem cells. Cell Stem Cell. 2013;13(5):535-48. Epub 2013/09/24. doi: 10.1016/j.stem.2013.08.012. PubMed PMID: 24054998.

Boisset JC, van Cappellen W, Andrieu-Soler C, Galjart N, Dzierzak E, Robin C. In vivo imaging of haematopoietic cells emerging from the mouse aortic endothelium. Nature. 2010;464(7285):116-20. Epub 2010/02/16. doi: 10.1038/nature08764. PubMed PMID: 20154729.

Boisset JC, Clapes T, Klaus A, Papazian N, Onderwater J, Mommaas-Kienhuis M, et al. Progressive maturation toward hematopoietic stem cells in the mouse embryo aorta. Blood. 2015;125(3):465-9. Epub 2014/10/11. doi: 10.1182/blood-2014-07-588954. PubMed PMID: 25301706; PubMed Central PMCID: PMCPMC4296008.

Boveri T. Anat. Anz. 1887;(2):688-693.

Boveri T. Befruchtung. 1892a; In Ergebnisse der Anatomie und Entwicklungsgeschichte, F.S. Merkel and R. Bonnet, eds. (Wiesbaden: Joseph Friedrich Bergmann), pp. 386-485.

Boveri T. Sitzungsber. d. Gesellschaft f. Morphologie und Physiologie 1892b;(8):114225.

Bowers M, Zhang B, Ho Y, Agarwal P, Chen CC, Bhatia R. Osteoblast ablation reduces normal long-term hematopoietic stem cell self-renewal but accelerates leukemia development. Blood. 2015;125(17):2678-88. Epub 2015/03/07. doi: 10.1182/blood-201406-582924. PubMed PMID: 25742698; PubMed Central PMCID: PMCPMC4408292.

Bowie MB, McKnight KD, Kent DG, McCaffrey L, Hoodless PA, Eaves CJ. Hematopoietic stem cells proliferate until after birth and show a reversible phase-specific engraftment defect. J Clin Invest. 2006;116(10):2808-16. Epub 2006/10/04. doi: 
10.1172/JCI28310. PubMed PMID: 17016561; PubMed Central PMCID: PMCPMC1578623.

Bowie MB, Kent DG, Dykstra B, McKnight KD, McCaffrey L, Hoodless PA, et al. Identification of a new intrinsically timed developmental checkpoint that reprograms key hematopoietic stem cell properties. Proc Natl Acad Sci U S A. 2007;104(14):5878-82. Epub 2007/03/24. doi: 10.1073/pnas.0700460104. PubMed PMID: 17379664; PubMed Central PMCID: PMCPMC1829493.

Boyer SW, Schroeder AV, Smith-Berdan S, Forsberg EC. All hematopoietic cells develop from hematopoietic stem cells through Flk2/Flt3-positive progenitor cells. Cell Stem Cell. 2011;9(1):64-73. Epub 2011/07/06. doi: 10.1016/j.stem.2011.04.021. PubMed PMID: 21726834; PubMed Central PMCID: PMCPMC4103692.

Bradley TR, Metcalf D. The growth of mouse bone marrow cells in vitro. Aust J Exp Biol Med Sci. 1966 Jun;44(3):287-99. doi: 10.1038/icb.1966.28. PMID: 4164182 Bruce WR, Meeker BE, Valeriote FA. Comparison of the sensitivity of normal hematopoietic and transplanted lymphoma colony-forming cells to chemotherapeutic agents administered in vivo. J Natl Cancer Inst. 1966 Aug;37(2):233-45. PMID: 5912615.

Bruns I, Lucas D, Pinho S, Ahmed J, Lambert MP, Kunisaki Y, et al. Megakaryocytes regulate hematopoietic stem cell quiescence through CXCL4 secretion. Nat Med. 2014;20(11):1315-20. Epub 2014/10/20. doi: 10.1038/nm.3707. PubMed PMID: 25326802; PubMed Central PMCID: PMCPMC4258871.

Buenrostro JD, Corces MR, Lareau CA, Wu B, Schep AN, Aryee MJ, et al. Integrated Single-Cell Analysis Maps the Continuous Regulatory Landscape of Human Hematopoietic Differentiation. Cell. 2018;173(6):1535-48 e16. Epub 2018/05/01. doi: 10.1016/j.cell.2018.03.074. PubMed PMID: 29706549; PubMed Central PMCID: PMCPMC5989727.

Burns CE, Traver D, Mayhall E, Shepard JL, Zon LI. Hematopoietic stem cell fate is established by the Notch-Runx pathway. Genes Dev. 2005;19(19):2331-42. Epub 2005/09/17. doi: 10.1101/gad.1337005. PubMed PMID: 16166372; PubMed Central PMCID: PMCPMC1240042.

Butler A, Hoffman P, Smibert P, Papalexi E, Satija R. Integrating single-cell transcriptomic data across different conditions, technologies, and species. Nat Biotechnol. 2018;36(5):411-20. Epub 2018/04/03. doi: 10.1038/nbt.4096. PubMed PMID: 29608179; PubMed Central PMCID: PMCPMC6700744.

Butler JM, Nolan DJ, Vertes EL, Varnum-Finney B, Kobayashi H, Hooper AT, et al. Endothelial cells are essential for the self-renewal and repopulation of Notch-dependent hematopoietic stem cells. Cell Stem Cell. 2010;6(3):251-64. Epub 2010/03/09. doi: 10.1016/j.stem.2010.02.001. PubMed PMID: 20207228; PubMed Central PMCID: PMCPMC2866527. 
Butler JM, Gars EJ, James DJ, Nolan DJ, Scandura JM, Rafii S. Development of a vascular niche platform for expansion of repopulating human cord blood stem and progenitor cells. Blood. 2012;120(6):1344-7. Epub 2012/06/20. doi: 10.1182/blood-201112-398115. PubMed PMID: 22709690; PubMed Central PMCID: PMCPMC3418723.

Cabezas-Wallscheid N, Klimmeck D, Hansson J, Lipka DB, Reyes A, Wang Q, et al. Identification of regulatory networks in HSCs and their immediate progeny via integrated proteome, transcriptome, and DNA methylome analysis. Cell Stem Cell. 2014;15(4):50722. Epub 2014/08/28. doi: 10.1016/j.stem.2014.07.005. PubMed PMID: 25158935.

Cai S, Tsui YP, Tam KW, Shea GK, Chang RS, Ao Q, et al. Directed Differentiation of Human Bone Marrow Stromal Cells to Fate-Committed Schwann Cells. Stem Cell Reports. 2017;9(4):1097-108. Epub 2017/09/12. doi: 10.1016/j.stemcr.2017.08.004. PubMed PMID: 28890164; PubMed Central PMCID: PMCPMC5639182.

Carrelha J, Meng Y, Kettyle LM, Luis TC, Norfo R, Alcolea V, et al. Hierarchically related lineage-restricted fates of multipotent haematopoietic stem cells. Nature. 2018;554(7690):106-11. Epub 2018/01/04. doi: 10.1038/nature25455. PubMed PMID: 29298288.

Casanova-Acebes M, Pitaval C, Weiss LA, Nombela-Arrieta C, Chevre R, N AG, et al. Rhythmic modulation of the hematopoietic niche through neutrophil clearance. Cell. 2013;153(5):1025-35. Epub 2013/05/28. doi: 10.1016/j.cell.2013.04.040. PubMed PMID: 23706740 ; PubMed Central PMCID: PMCPMC4128329.

Chapple RH, Tseng YJ, Hu T, Kitano A, Takeichi M, Hoegenauer KA, et al. Lineage tracing of murine adult hematopoietic stem cells reveals active contribution to steadystate hematopoiesis. Blood Adv. 2018;2(11):1220-8. Epub 2018/06/01. doi: 10.1182/bloodadvances.2018016295. PubMed PMID: 29848758; PubMed Central PMCID: PMCPMC5998934.

Chen MJ, Yokomizo T, Zeigler BM, Dzierzak E, Speck NA. Runx1 is required for the endothelial to haematopoietic cell transition but not thereafter. Nature.

2009;457(7231):887-91. Epub 2009/01/09. doi: 10.1038/nature07619. PubMed PMID: 19129762; PubMed Central PMCID: PMCPMC2744041.

Chen MJ, Li Y, De Obaldia ME, Yang Q, Yzaguirre AD, Yamada-Inagawa T, et al. Erythroid/myeloid progenitors and hematopoietic stem cells originate from distinct populations of endothelial cells. Cell Stem Cell. 2011;9(6):541-52. Epub 2011/12/06. doi: 10.1016/j.stem.2011.10.003. PubMed PMID: 22136929; PubMed Central PMCID: PMCPMC3576591.

Chen X, Deng H, Churchill MJ, Luchsinger LL, Du X, Chu TH, et al. Bone Marrow Myeloid Cells Regulate Myeloid-Biased Hematopoietic Stem Cells via a HistamineDependent Feedback Loop. Cell Stem Cell. 2017;21(6):747-60 e7. Epub 2017/12/05. doi: 
10.1016/j.stem.2017.11.003. PubMed PMID: 29198940; PubMed Central PMCID: PMCPMC5975960.

Chhiba KD, Hsu CL, Berdnikovs S, Bryce PJ. Transcriptional Heterogeneity of Mast Cells and Basophils upon Activation. J Immunol. 2017;198(12):4868-78. Epub 2017/05/10. doi: 10.4049/jimmunol.1601825. PubMed PMID: 28476932; PubMed Central PMCID: PMCPMC5862545.

Choi K, Kennedy M, Kazarov A, Papadimitriou JC, Keller G. A common precursor for hematopoietic and endothelial cells. Development. 1998 Feb;125(4):725-32. PMID: 9435292.

Chou S, Lodish HF. Fetal liver hepatic progenitors are supportive stromal cells for hematopoietic stem cells. Proc Natl Acad Sci U S A. 2010;107(17):7799-804. Epub 2010/04/14. doi: 10.1073/pnas.1003586107. PubMed PMID: 20385801; PubMed Central PMCID: PMCPMC2867886.

Chow A, Lucas D, Hidalgo A, Mendez-Ferrer S, Hashimoto D, Scheiermann C, et al. Bone marrow CD169+ macrophages promote the retention of hematopoietic stem and progenitor cells in the mesenchymal stem cell niche. J Exp Med. 2011;208(2):261-71. Epub 2011/02/02. doi: 10.1084/jem.20101688. PubMed PMID: 21282381; PubMed Central PMCID: PMCPMC3039855.

Christensen JL, Wright DE, Wagers AJ, Weissman IL. Circulation and chemotaxis of fetal hematopoietic stem cells. PLoS Biol. 2004;2(3):E75. Epub 2004/03/17. doi: 10.1371/journal.pbio.0020075. PubMed PMID: 15024423; PubMed Central PMCID: PMCPMC368169.

Christopher MJ, Rao M, Liu F, Woloszynek JR, Link DC. Expression of the G-CSF receptor in monocytic cells is sufficient to mediate hematopoietic progenitor mobilization by G-CSF in mice. J Exp Med. 2011;208(2):251-60. Epub 2011/02/02. doi: 10.1084/jem.20101700. PubMed PMID: 21282380; PubMed Central PMCID: PMCPMC3039862.

Ciau-Uitz A, Pinheiro P, Gupta R, Enver T, Patient R. Tel1/ETV6 specifies blood stem cells through the agency of VEGF signaling. Dev Cell. 2010;18(4):569-78. Epub 2010/04/24. doi: 10.1016/j.devcel.2010.02.009. PubMed PMID: 20412772.

Ciriza J, Thompson H, Petrosian R, Manilay JO, Garcia-Ojeda ME. The migration of hematopoietic progenitors from the fetal liver to the fetal bone marrow: lessons learned and possible clinical applications. Exp Hematol. 2013;41(5):411-23. Epub 2013/02/12. doi: 10.1016/j.exphem.2013.01.009. PubMed PMID: 23395775.

Cleaver O, Krieg PA. VEGF mediates angioblast migration during development of the dorsal aorta in Xenopus. Development. 1998 Oct;125(19):3905-14. PMID: 9729498. 
Coffman RL, Weissman IL. A monoclonal antibody that recognizes B cells and B cell precursors in mice. J Exp Med. 1981a;153(2):269-79. Epub 1981/02/01. doi: 10.1084/jem.153.2.269. PubMed PMID: 6787164; PubMed Central PMCID: PMCPMC2186081.

Coffman RL, Weissman IL. B220: a B cell-specific member of th T200 glycoprotein family. Nature. 1981b;289(5799):681-3. Epub 1981/02/19. doi: 10.1038/289681a0. PubMed PMID: 6970340.

Cooper B. The origins of bone marrow as the seedbed of our blood: from antiquity to the time of Osler. Proc (Bayl Univ Med Cent). 2011;24(2):115-8. Epub 2011/05/14. doi: 10.1080/08998280.2011.11928697. PubMed PMID: 21566758; PubMed Central PMCID: PMCPMC3069519.

Cortegano I, Serrano N, Ruiz C, Rodriguez M, Prado C, Alia M, et al. CD45 expression discriminates waves of embryonic megakaryocytes in the mouse. Haematologica. 2019;104(9):1853-65. Epub 2018/12/24. doi: 10.3324/haematol.2018.192559. PubMed PMID: 30573502; PubMed Central PMCID: PMCPMC6717566.

Coskun S, Chao H, Vasavada H, Heydari K, Gonzales N, Zhou X, et al. Development of the fetal bone marrow niche and regulation of HSC quiescence and homing ability by emerging osteolineage cells. Cell Rep. 2014;9(2):581-90. Epub 2014/10/15. doi: 10.1016/j.celrep.2014.09.013. PubMed PMID: 25310984; PubMed Central PMCID: PMCPMC4266564.

Craig ML, Russell ES. A developmental change in hemoglobins correlated with an embryonic red cell population in the mouse. Developmental Biology. 1964;10(2):191201. doi: 10.1016/0012-1606(64)90040-5.

Crane GM, Jeffery E, Morrison SJ. Adult haematopoietic stem cell niches. Nat Rev Immunol. 2017;17(9):573-90. Epub 2017/06/13. doi: 10.1038/nri.2017.53. PubMed PMID: 28604734.

Cumano A, Ferraz JC, Klaine M, Di Santo JP, Godin I. Intraembryonic, but Not Yolk Sac Hematopoietic Precursors, Isolated before Circulation, Provide Long-Term Multilineage Reconstitution. Immunity. 2001;15(3):477-85. doi: 10.1016/s1074-7613(01)00190-x.

Dahlin JS, Hallgren J. Mast cell progenitors: origin, development and migration to tissues. Mol Immunol. 2015;63(1):9-17. Epub 2014/03/07. doi: 10.1016/j.molimm.2014.01.018. PubMed PMID: 24598075.

Dahlin JS, Hamey FK, Pijuan-Sala B, Shepherd M, Lau WWY, Nestorowa S, et al. A single-cell hematopoietic landscape resolves 8 lineage trajectories and defects in Kit mutant mice. Blood. 2018;131(21):e1-e11. Epub 2018/03/29. doi: 10.1182/blood-201712-821413. PubMed PMID: 29588278; PubMed Central PMCID: PMCPMC5969381. 
Dantschakoff W. Anat. Hefte 1908;(37):472-589.

de Bruijn MF, Speck NA, Peeters MC, Dzierzak E. Definitive hematopoietic stem cells first develop within the major arterial regions of the mouse embryo. EMBO J.

2000;19(11):2465-74. Epub 2000/06/03. doi: 10.1093/emboj/19.11.2465. PubMed PMID: 10835345; PubMed Central PMCID: PMCPMC212758.

de Bruijn MFTR, Ma X, Robin C, Ottersbach K, Sanchez M-J, Dzierzak E. Hematopoietic Stem Cells Localize to the Endothelial Cell Layer in the Midgestation Mouse Aorta. Immunity. 2002;16(5):673-83. doi: 10.1016/s1074-7613(02)00313-8.

de Graaf CA, Choi J, Baldwin TM, Bolden JE, Fairfax KA, Robinson AJ, et al. Haemopedia: An Expression Atlas of Murine Hematopoietic Cells. Stem Cell Reports. 2016;7(3):571-82. Epub 2016/08/09. doi: 10.1016/j.stemcr.2016.07.007. PubMed PMID: 27499199; PubMed Central PMCID: PMCPMC5031953.

Dexter TM, Allen TD, Lajtha LG. Conditions controlling the proliferation of haemopoietic stem cells in vitro. J Cell Physiol. 1977;91(3):335-44. Epub 1977/06/01. doi: 10.1002/jcp.1040910303. PubMed PMID: 301143.

Dieterlen-Lievre F. On the origin of haemopoietic stem cells in the avian embryo: an experimental approach. J Embryol Exp Morphol. 1975 Jun;33(3):607-19. PMID: 1176862.

Ding L, Saunders TL, Enikolopov G, Morrison SJ. Endothelial and perivascular cells maintain haematopoietic stem cells. Nature. 2012;481(7382):457-62. Epub 2012/01/28. doi: 10.1038/nature10783. PubMed PMID: 22281595; PubMed Central PMCID: PMCPMC3270376.

Dominguez-Bello MG, Costello EK, Contreras M, Magris M, Hidalgo G, Fierer N, et al. Delivery mode shapes the acquisition and structure of the initial microbiota across multiple body habitats in newborns. Proc Natl Acad Sci U S A. 2010;107(26):11971-5. Epub 2010/06/23. doi: 10.1073/pnas.1002601107. PubMed PMID: 20566857; PubMed Central PMCID: PMCPMC2900693.

Dooley KA, Davidson AJ, Zon LI. Zebrafish scl functions independently in hematopoietic and endothelial development. Dev Biol. 2005;277(2):522-36. Epub 2004/12/25. doi: 10.1016/j.ydbio.2004.09.004. PubMed PMID: 15617691.

Drake CJ, Fleming PA. Vasculogenesis in the day 6.5 to 9.5 mouse embryo. Blood. 2000;95(5):1671-9. doi: 10.1182/blood.V95.5.1671.005k39_1671_1679.

Dwyer DF, Barrett NA, Austen KF, Immunological Genome Project C. Expression profiling of constitutive mast cells reveals a unique identity within the immune system. Nat Immunol. 2016;17(7):878-87. Epub 2016/05/03. doi: 10.1038/ni.3445. PubMed PMID: 27135604; PubMed Central PMCID: PMCPMC5045264. 
Dykstra B, Kent D, Bowie M, McCaffrey L, Hamilton M, Lyons K, et al. Long-term propagation of distinct hematopoietic differentiation programs in vivo. Cell Stem Cell. 2007;1(2):218-29. Epub 2008/03/29. doi: 10.1016/j.stem.2007.05.015. PubMed PMID: 18371352.

Eaves CJ. Hematopoietic stem cells: concepts, definitions, and the new reality. Blood. 2015;125(17):2605-13. Epub 2015/03/13. doi: 10.1182/blood-2014-12-570200. PubMed PMID: 25762175; PubMed Central PMCID: PMCPMC4440889.

Ehrlich P. Arch. Anat. u. Physiol. 1879;(3):571-579.

Eichmann A, Corbel C, Nataf V, Vaigot P, Breant C, Le Douarin NM. Ligand-dependent development of the endothelial and hemopoietic lineages from embryonic mesodermal cells expressing vascular endothelial growth factor receptor 2. Proc Natl Acad Sci U S A. 1997;94(10):5141-6. Epub 1997/05/13. doi: 10.1073/pnas.94.10.5141. PubMed PMID: 9144204; PubMed Central PMCID: PMCPMC24645.

Ema H, Nakauchi H. Expansion of hematopoietic stem cells in the developing liver of a mouse embryo. Blood. 2000;95(7):2284-8. doi: 10.1182/blood.V95.7.2284.

Ezine S, Weissman IL, Rouse RV. Bone marrow cells give rise to distinct cell clones within the thymus. Nature. 1984;309(5969):629-31. Epub 1984/06/14. doi: 10.1038/309629a0. PubMed PMID: 6374470.

Ferkowicz MJ, Yoder MC. Blood island formation: longstanding observations and modern interpretations. Exp Hematol. 2005;33(9):1041-7. Epub 2005/09/06. doi: 10.1016/j.exphem.2005.06.006. PubMed PMID: 16140152.

Ford CE, Hamerton JL, Barnes DW, Loutit JF. Cytological identification of radiationchimaeras. Nature. 1956;177(4506):452-4. Epub 1956/03/10. doi: 10.1038/177452a0. PubMed PMID: 13309336.

Forsberg EC, Serwold T, Kogan S, Weissman IL, Passegue E. New evidence supporting megakaryocyte-erythrocyte potential of flk2/flt3 + multipotent hematopoietic progenitors. Cell. 2006;126(2):415-26. Epub 2006/07/29. doi: 10.1016/j.cell.2006.06.037. PubMed PMID: 16873070.

Fouquet B, Weinstein BM, Serluca FC, Fishman MC. Vessel patterning in the embryo of the zebrafish: guidance by notochord. Dev Biol. 1997;183(1):37-48. Epub 1997/03/01. doi: 10.1006/dbio.1996.8495. PubMed PMID: 9119113.

Fujisaki J, Wu J, Carlson AL, Silberstein L, Putheti P, Larocca R, et al. In vivo imaging of Treg cells providing immune privilege to the haematopoietic stem-cell niche. Nature. 2011;474(7350):216-9. Epub 2011/06/10. doi: 10.1038/nature10160. PubMed PMID: 21654805; PubMed Central PMCID: PMCPMC3725645. 
Ganuza M, Hall T, Finkelstein D, Chabot A, Kang G, McKinney-Freeman S. Lifelong haematopoiesis is established by hundreds of precursors throughout mammalian ontogeny. Nat Cell Biol. 2017;19(10):1153-63. Epub 2017/09/19. doi: 10.1038/ncb3607. PubMed PMID: 28920953; PubMed Central PMCID: PMCPMC5705075.

Ganuza M, Chabot A, Tang X, Bi W, Natarajan S, Carter R, et al. Murine hematopoietic stem cell activity is derived from pre-circulation embryos but not yolk sacs. Nat Commun. 2018;9(1):5405. Epub 2018/12/24. doi: 10.1038/s41467-018-07769-8. PubMed PMID: 30573729; PubMed Central PMCID: PMCPMC6302089.

Gao X, Xu C, Asada N, Frenette PS. The hematopoietic stem cell niche: from embryo to adult. Development. 2018;145(2). Epub 2018/01/24. doi: 10.1242/dev.139691. PubMed PMID: 29358215; PubMed Central PMCID: PMCPMC5825844.

Gekas C, Dieterlen-Lievre F, Orkin SH, Mikkola HK. The placenta is a niche for hematopoietic stem cells. Dev Cell. 2005;8(3):365-75. Epub 2005/03/02. doi: 10.1016/j.devcel.2004.12.016. PubMed PMID: 15737932.

Gering M, Rodaway AR, Gottgens B, Patient RK, Green AR. The SCL gene specifies haemangioblast development from early mesoderm. EMBO J. 1998;17(14):4029-45. Epub 1998/07/22. doi: 10.1093/emboj/17.14.4029. PubMed PMID: 9670018; PubMed Central PMCID: PMCPMC1170736.

Gering M, Patient R. Hedgehog signaling is required for adult blood stem cell formation in zebrafish embryos. Dev Cell. 2005;8(3):389-400. Epub 2005/03/02. doi:

10.1016/j.devcel.2005.01.010. PubMed PMID: 15737934.

Giladi A, Paul F, Herzog Y, Lubling Y, Weiner A, Yofe I, et al. Single-cell characterization of haematopoietic progenitors and their trajectories in homeostasis and perturbed haematopoiesis. Nat Cell Biol. 2018;20(7):836-46. Epub 2018/06/20. doi: 10.1038/s41556-018-0121-4. PubMed PMID: 29915358.

Goncalves KA, Silberstein L, Li S, Severe N, Hu MG, Yang H, et al. Angiogenin Promotes Hematopoietic Regeneration by Dichotomously Regulating Quiescence of Stem and Progenitor Cells. Cell. 2016;166(4):894-906. Epub 2016/08/16. doi: 10.1016/j.cell.2016.06.042. PubMed PMID: 27518564; PubMed Central PMCID: PMCPMC4988404.

Goodell MA, Brose K, Paradis G, Conner AS, Mulligan RC. Isolation and functional properties of murine hematopoietic stem cells that are replicating in vivo. J Exp Med. 1996;183(4):1797-806. Epub 1996/04/01. doi: 10.1084/jem.183.4.1797. PubMed PMID: 8666936; PubMed Central PMCID: PMCPMC2192511.

Gordon-Keylock S, Sobiesiak M, Rybtsov S, Moore K, Medvinsky A. Mouse extraembryonic arterial vessels harbor precursors capable of maturing into definitive 
HSCs. Blood. 2013;122(14):2338-45. Epub 2013/07/19. doi: 10.1182/blood-2012-12470971. PubMed PMID: 23863896; PubMed Central PMCID: PMCPMC3790504.

Gori JL, Butler JM, Kunar B, Poulos MG, Ginsberg M, Nolan DJ, et al. Endothelial Cells Promote Expansion of Long-Term Engrafting Marrow Hematopoietic Stem and Progenitor Cells in Primates. Stem Cells Transl Med. 2017;6(3):864-76. Epub 2017/03/16. doi: 10.5966/sctm.2016-0240. PubMed PMID: 28297579; PubMed Central PMCID: PMCPMC5442761.

Greenbaum A, Hsu YM, Day RB, Schuettpelz LG, Christopher MJ, Borgerding JN, et al. CXCL12 in early mesenchymal progenitors is required for haematopoietic stem-cell maintenance. Nature. 2013;495(7440):227-30. Epub 2013/02/26. doi: 10.1038/nature1 1926. PubMed PMID: 23434756; PubMed Central PMCID: PMCPMC3600148.

Gregory CJ, McCulloch EA, Till JE. Erythropoietic progenitors capable of colony formation in culture: state of differentiation. J Cell Physiol. 1973;81(3):411-20. Epub 1973/06/01. doi: 10.1002/jcp.1040810313. PubMed PMID: 4713721.

Gregory CJ. Erythropoietin sensitivity as a differentiation marker in the hemopoietic system: studies of three erythropoietic colony responses in culture. J Cell Physiol. 1976;89(2):289-301. Epub 1976/10/01. doi: 10.1002/jcp.1040890212. PubMed PMID: 987043.

Haas S, Hansson J, Klimmeck D, Loeffler D, Velten L, Uckelmann H, et al. Inflammation-Induced Emergency Megakaryopoiesis Driven by Hematopoietic Stem Cell-like Megakaryocyte Progenitors. Cell Stem Cell. 2015;17(4):422-34. Epub 2015/08/25. doi: 10.1016/j.stem.2015.07.007. PubMed PMID: 26299573.

Häcker V. Archiv f. mikr. Anat. 1892;(39):556-581.

Hadland BK, Huppert SS, Kanungo J, Xue Y, Jiang R, Gridley T, et al. A requirement for Notch1 distinguishes 2 phases of definitive hematopoiesis during development. Blood. 2004;104(10):3097-105. Epub 2004/07/15. doi: 10.1182/blood-2004-03-1224. PubMed PMID: 15251982; PubMed Central PMCID: PMCPMC5998659.

Haeckel E. Natürliche Schöpfungsgeschichte 1868 (Berlin: Georg Reimer).

Haeckel E. Anthropogenie, 1st edn 1874 (Leipzig:Wilhelm Engelmann).

Haeckel E. Anthropogenie, 3rd edn 1877 (Leipzig:Wilhelm Engelmann).

Haferkamp I, Schmitz-Esser S, Linka N, Urbany C, Collingro A, Wagner M, et al. A candidate NAD + transporter in an intracellular bacterial symbiont related to Chlamydiae. Nature. 2004;432(7017):622-5. Epub 2004/12/04. doi: 10.1038/nature03131. PubMed PMID: 15577910. 
Hall BK, Miyake T. All for one and one for all: condensations and the initiation of skeletal development. BioEssays. 2000;22(2):138-47. doi: 10.1002/(sici)15211878(200002)22:2<138::Aid-bies5>3.0.Co;2-4.

Hamey FK, Gottgens B. Machine learning predicts putative hematopoietic stem cells within large single-cell transcriptomics data sets. Exp Hematol. 2019;78:11-20. Epub 2019/09/13. doi: 10.1016/j.exphem.2019.08.009. PubMed PMID: 31513832; PubMed Central PMCID: PMCPMC6900257.

Harrison DE. Competitive repopulation: a new assay for long-term stem cell functional capacity. Blood. 1980;55(1):77-81. doi: 10.1182/blood.V55.1.77.77.

Harrison DE, Zhong RK, Jordan CT, Lemischka IR, Astle CM. Relative to adult marrow, fetal liver repopulates nearly five times more effectively long-term than short-term. Exp Hematol. 1997 Apr;25(4):293-7. PMID: 9131003.

Henninger J, Santoso B, Hans S, Durand E, Moore J, Mosimann C, et al. Clonal fate mapping quantifies the number of haematopoietic stem cells that arise during development. Nat Cell Biol. 2017;19(1):17-27. Epub 2016/11/22. doi: 10.1038/ncb3444. PubMed PMID: 27870830; PubMed Central PMCID: PMCPMC5535785.

Hodgson GS, Bradley TR. Properties of haematopoietic stem cells surviving 5fluorouracil treatment: evidence for a pre-CFU-S cell? Nature. 1979;281(5730):381-2 . Epub 1979/10/04. doi: 10.1038/281381a0. PubMed PMID: 481601.

Houssaint E. Differentiation of the mouse hepatic primordium. II. Extrinsic origin of the haemopoietic cell line. Cell Differentiation. 1981;10(5):243-52. doi: 10.1016/00456039(81)90007-5.

Ikuta K, Weissman IL. Evidence that hematopoietic stem cells express mouse c-kit but do not depend on steel factor for their generation. Proc Natl Acad Sci U S A. 1992;89(4):1502-6. Epub 1992/02/15. doi: 10.1073/pnas.89.4.1502. PubMed PMID: 1371359; PubMed Central PMCID: PMCPMC48479.

Isern J, Fraser ST, He Z, Baron MH. The fetal liver is a niche for maturation of primitive erythroid cells. Proc Natl Acad Sci U S A. 2008;105(18):6662-7. Epub 2008/05/01. doi: 10.1073/pnas.0802032105. PubMed PMID: 18445648; PubMed Central PMCID: PMCPMC2373346.

Isern J, Garcia-Garcia A, Martin AM, Arranz L, Martin-Perez D, Torroja C, et al. The neural crest is a source of mesenchymal stem cells with specialized hematopoietic stem cell niche function. Elife. 2014;3:e03696. Epub 2014/09/26. doi: 10.7554/eLife.03696. PubMed PMID: 25255216; PubMed Central PMCID: PMCPMC4381911.

Itkin T, Gur-Cohen S, Spencer JA, Schajnovitz A, Ramasamy SK, Kusumbe AP, et al. Distinct bone marrow blood vessels differentially regulate haematopoiesis. Nature. 
2016;532(7599):323-8. Epub 2016/04/14. doi: 10.1038/nature17624. PubMed PMID: 27074509; PubMed Central PMCID: PMCPMC6450701

Jacobson LO, Simmons EL, Marks EK, Robson MJ, Bethard WF, Gaston EO. The role of the spleen in radiation injury and recovery. J Lab Clin Med. 1950 May;35(5):746-70. PMID: 15422209.

Jaffredo T, Gautier R, Eichmann A, Dieterlen-Lièvre F. Intraaortic hemopoietic cells are derived from endothelial cells during ontogeny. Development. 1998 Nov;125(22):457583. PMID: 9778515.

Johnson GR, Moore MA. Role of stem cell migration in initiation of mouse foetal liver haemopoiesis. Nature. 1975;258(5537):726-8. Epub 1975/12/25. doi: 10.1038/258726a0. PubMed PMID: 1207754.

Josefsdottir KS, Baldridge MT, Kadmon CS, King KY. Antibiotics impair murine hematopoiesis by depleting the intestinal microbiota. Blood. 2017;129(6):729-39. Epub 2016/11/24. doi: 10.1182/blood-2016-03-708594. PubMed PMID: 27879260; PubMed Central PMCID: PMCPMC5301822.

Katayama Y, Battista M, Kao WM, Hidalgo A, Peired AJ, Thomas SA, et al. Signals from the sympathetic nervous system regulate hematopoietic stem cell egress from bone marrow. Cell. 2006;124(2):407-21. Epub 2006/01/28. doi: 10.1016/j.cell.2005.10.041. PubMed PMID: 16439213.

Kawamoto H, Ikawa T, Ohmura K, Fujimoto S, Katsura Y. T Cell Progenitors Emerge Earlier Than B Cell Progenitors in the Murine Fetal Liver. Immunity. 2000;12(4):441-50. doi: 10.1016/s1074-7613(00)80196-x.

Kennedy M, D'Souza SL, Lynch-Kattman M, Schwantz S, Keller G. Development of the hemangioblast defines the onset of hematopoiesis in human ES cell differentiation cultures. Blood. 2007;109(7):2679-87. Epub 2006/12/07. doi: 10.1182/blood-2006-09047704. PubMed PMID: 17148580; PubMed Central PMCID: PMCPMC1852226.

Kent DG, Copley MR, Benz C, Wohrer S, Dykstra BJ, Ma E, et al. Prospective isolation and molecular characterization of hematopoietic stem cells with durable self-renewal potential. Blood. 2009;113(25):6342-50. Epub 2009/04/21. doi: 10.1182/blood-2008-12192054. PubMed PMID: 19377048.

Khan JA, Mendelson A, Kunisaki Y, Birbrair A, Kou Y, Arnal-Estape A, et al. Fetal liver hematopoietic stem cell niches associate with portal vessels. Science.

2016;351(6269):176-80. Epub 2015/12/05. doi: 10.1126/science.aad0084. PubMed PMID: 26634440; PubMed Central PMCID: PMCPMC4706788. 
Kiel MJ, Yilmaz OH, Iwashita T, Yilmaz OH, Terhorst C, Morrison SJ. SLAM family receptors distinguish hematopoietic stem and progenitor cells and reveal endothelial niches for stem cells. Cell. 2005;121(7):1109-21. Epub 2005/07/02. doi:

10.1016/j.cell.2005.05.026. PubMed PMID: 15989959.

Kim I, Yilmaz OH, Morrison SJ. CD144 (VE-cadherin) is transiently expressed by fetal liver hematopoietic stem cells. Blood. 2005;106(3):903-5. Epub 2005/04/16. doi: 10.1182/blood-2004-12-4960. PubMed PMID: 15831702; PubMed Central PMCID: PMCPMC1895147.

Kinder SJ, Tsang TE, Quinlan GA, Hadjantonakis AK, Nagy A, Tam PP. The orderly allocation of mesodermal cells to the extraembryonic structures and the anteroposterior axis during gastrulation of the mouse embryo. Development. 1999 Nov;126(21):4691701. PMID: 10518487.

Kingsley PD, Malik J, Fantauzzo KA, Palis J. Yolk sac-derived primitive erythroblasts enucleate during mammalian embryogenesis. Blood. 2004;104(1):19-25. Epub 2004/03/20. doi: 10.1182/blood-2003-12-4162. PubMed PMID: 15031208.

Kissa K, Herbomel P. Blood stem cells emerge from aortic endothelium by a novel type of cell transition. Nature. 2010;464(7285):112-5. Epub 2010/02/16. doi:

10.1038/nature08761. PubMed PMID: 20154732.

Kobayashi M, Shelley WC, Seo W, Vemula S, Lin Y, Liu Y, et al. Functional B-1 progenitor cells are present in the hematopoietic stem cell-deficient embryo and depend on Cbfbeta for their development. Proc Natl Acad Sci U S A. 2014a;111(33):12151-6. Epub 2014a/08/06. doi: 10.1073/pnas.1407370111. PubMed PMID: 25092306; PubMed Central PMCID: PMCPMC4143017.

Kobayashi T, Kronenberg HM. Overview of skeletal development. Methods Mol Biol. 2014;1130:3-12. Epub 2014b/02/01. doi: 10.1007/978-1-62703-989-5_1. PubMed PMID: 24482161 .

Kondo M, Weissman IL, Akashi K. Identification of Clonogenic Common Lymphoid Progenitors in Mouse Bone Marrow. Cell. 1997;91(5):661-72. doi: 10.1016/s00928674(00)80453-5.

Kowalczyk MS, Tirosh I, Heckl D, Rao TN, Dixit A, Haas BJ, et al. Single-cell RNA-seq reveals changes in cell cycle and differentiation programs upon aging of hematopoietic stem cells. Genome Res. 2015;25(12):1860-72. Epub 2015/10/03. doi: 10.1101/gr.192237.115. PubMed PMID: 26430063; PubMed Central PMCID: PMCPMC4665007.

Kumano K, Chiba S, Kunisato A, Sata M, Saito T, Nakagami-Yamaguchi E, et al. Notch1 but Not Notch2 Is Essential for Generating Hematopoietic Stem Cells from Endothelial Cells. Immunity. 2003;18(5):699-711. doi: 10.1016/s1074-7613(03)00117-1. 
Kumaravelu P, Hook L, Morrison AM, Ure J, Zhao S, Zuyev S, et al. Quantitative developmental anatomy of definitive haematopoietic stem cells/long-term repopulating units (HSC/RUs): role of the aorta-gonad-mesonephros (AGM) region and the yolk sac in colonisation of the mouse embryonic liver. Development. 2002 Nov;129(21):4891-9. Erratum in: Development. 2003 Jan;130(2):425. PMID: 12397098.

Kunisaki Y, Bruns I, Scheiermann C, Ahmed J, Pinho S, Zhang D, et al. Arteriolar niches maintain haematopoietic stem cell quiescence. Nature. 2013;502(7473):637-43. Epub 2013/10/11. doi: 10.1038/nature12612. PubMed PMID: 24107994; PubMed Central PMCID: PMCPMC3821873.

Lambertsen RH, Weiss L. A model of intramedullary hematopoietic microenvironments based on stereologic study of the distribution of endocloned marrow colonies. Blood. 1984;63(2):287-97. doi: 10.1182/blood.V63.2.287.287.

Lancrin C, Sroczynska P, Stephenson C, Allen T, Kouskoff V, Lacaud G. The haemangioblast generates haematopoietic cells through a haemogenic endothelium stage. Nature. 2009;457(7231):892-5. Epub 2009/02/03. doi: 10.1038/nature07679. PubMed PMID: 19182774; PubMed Central PMCID: PMCPMC2661201.

Langen UH, Pitulescu ME, Kim JM, Enriquez-Gasca R, Sivaraj KK, Kusumbe AP, et al. Cell-matrix signals specify bone endothelial cells during developmental osteogenesis. Nat Cell Biol. 2017;19(3):189-201. Epub 2017/02/22. doi: 10.1038/ncb3476. PubMed PMID: 28218908 ; PubMed Central PMCID: PMCPMC5580829.

Lawson ND, Scheer N, Pham VN, Kim CH, Chitnis AB, Campos-Ortega JA, et al. Notch signaling is required for arterial-venous differentiation during embryonic vascular development. Development. 2001 Oct;128(19):3675-83. PMID: 11585794.

Lepault F, Weissman IL. An in vivo assay for thymus-homing bone marrow cells. Nature. 1981;293(5828):151-4. Epub 1981/09/10. doi: 10.1038/293151a0. PubMed PMID: 6115320.

Liakhovitskaia A, Rybtsov S, Smith T, Batsivari A, Rybtsova N, Rode C, et al. Runx1 is required for progression of CD41+ embryonic precursors into HSCs but not prior to this. Development. 2014;141(17):3319-23. Epub 2014/08/21. doi: 10.1242/dev.110841. PubMed PMID: 25139854; PubMed Central PMCID: PMCPMC4199125.

Lis R, Karrasch CC, Poulos MG, Kunar B, Redmond D, Duran JGB, et al. Conversion of adult endothelium to immunocompetent haematopoietic stem cells. Nature. 2017;545(7655):439-45. Epub 2017/05/18. doi: 10.1038/nature22326. PubMed PMID: 28514438 ; PubMed Central PMCID: PMCPMC5794215.

Lorenz E, Congdon C, Uphoff D. Modification of acute irradiation injury in mice and guinea-pigs by bone marrow injections. Radiology. 1952;58(6):863-77. Epub 1952/06/01. doi: 10.1148/58.6.863. PubMed PMID: 14941986. 
Lu R, Neff NF, Quake SR, Weissman IL. Tracking single hematopoietic stem cells in vivo using high-throughput sequencing in conjunction with viral genetic barcoding. Nat Biotechnol. 2011;29(10):928-33. Epub 2011/10/04. doi: 10.1038/nbt.1977. PubMed PMID: 21964413; PubMed Central PMCID: PMCPMC3196379.

Magli MC, Iscove NN, Odartchenko N. Transient nature of early haematopoietic spleen colonies. Nature. 1982;295(5849):527-9. Epub 1982/02/11. doi: 10.1038/295527a0.

PubMed PMID: 7057912.

Main JM PR. Successful Skin Homografts After the Administration of High Dosage X Radiation and Homologous Bone Marrow. JNCI: Journal of the National Cancer Institute. 1955. doi: 10.1093/jnci/15.4.1023.

Mantel CR, O'Leary HA, Chitteti BR, Huang X, Cooper S, Hangoc G, et al. Enhancing Hematopoietic Stem Cell Transplantation Efficacy by Mitigating Oxygen Shock. Cell. 2015;161(7):1553-65. Epub 2015/06/16. doi: 10.1016/j.cell.2015.04.054. PubMed PMID: 26073944; PubMed Central PMCID: PMCPMC4480616.

Martin MA, Bhatia M. Analysis of the human fetal liver hematopoietic microenvironment. Stem Cells Dev. 2005;14(5):493-504. Epub 2005/11/25. doi: 10.1089/scd.2005.14.493. PubMed PMID: 16305335.

Matsushita Y, Ono W, Ono N. Growth plate skeletal stem cells and their transition from cartilage to bone. Bone. 2020;136:115359. Epub 2020/04/11. doi: 10.1016/j.bone.2020.115359. PubMed PMID: 32276155; PubMed Central PMCID: PMCPMC7246136.

Maximow AA. Relation of Blood Cells to Connective Tissues and Endothelium. Physiological Reviews. 1924;4(4):533-63. doi: 10.1152/physrev.1924.4.4.533.

McCabe A, Zhang Y, Thai V, Jones M, Jordan MB, MacNamara KC. MacrophageLineage Cells Negatively Regulate the Hematopoietic Stem Cell Pool in Response to Interferon Gamma at Steady State and During Infection. Stem Cells. 2015;33(7):2294305. Epub 2015/04/17. doi: 10.1002/stem.2040. PubMed PMID: 25880153; PubMed Central PMCID: PMCPMC4693298.

McCulloch EA, Till JE. The radiation sensitivity of normal mouse bone marrow cells, determined by quantitative marrow transplantation into irradiated mice. Radiat Res. 1960 Jul;13:115-25. PMID: 13858509.

McGrath KE, Koniski AD, Malik J, Palis J. Circulation is established in a stepwise pattern in the mammalian embryo. Blood. 2003;101(5):1669-76. Epub 2002/10/31. doi: 10.1182/blood-2002-08-2531. PubMed PMID: 12406884.

McGrath KE, Kingsley PD, Koniski AD, Porter RL, Bushnell TP, Palis J. Enucleation of primitive erythroid cells generates a transient population of "pyrenocytes" in the 
mammalian fetus. Blood. 2008;111(4):2409-17. Epub 2007/11/23. doi: 10.1182/blood2007-08-107581. PubMed PMID: 18032705; PubMed Central PMCID: PMCPMC2234067.

McGrath KE, Frame JM, Fegan KH, Bowen JR, Conway SJ, Catherman SC, et al. Distinct Sources of Hematopoietic Progenitors Emerge before HSCs and Provide Functional Blood Cells in the Mammalian Embryo. Cell Rep. 2015;11(12):1892-904. Epub 2015/06/23. doi: 10.1016/j.celrep.2015.05.036. PubMed PMID: 26095363; PubMed Central PMCID: PMCPMC4490098.

McInnes L, Healy J, Melville J. UMAP: Uniform Manifold Approximation and Projection for dimension reduction. arXiv:1802.03426v2 [stat.ML] 6 Dec 2018

McKinney-Freeman SL, Jackson KA, Camargo FD, Ferrari G, Mavilio F, Goodell MA. Muscle-derived hematopoietic stem cells are hematopoietic in origin. Proc Natl Acad Sci U S A. 2002;99(3):1341-6. Epub 2002/02/07. doi: 10.1073/pnas.032438799. PubMed PMID: 11830662; PubMed Central PMCID: PMCPMC122192.

Medvinsky A, Dzierzak E. Definitive Hematopoiesis Is Autonomously Initiated by the AGM Region. Cell. 1996a;86(6):897-906. doi: 10.1016/s0092-8674(00)80165-8.

Medvinsky AL, Gan OI, Semenova ML, Samoylina NL. Development of day-8 colonyforming unit-spleen hematopoietic progenitors during early murine embryogenesis: spatial and temporal mapping. Blood. 1996b;87(2):557-66. doi: 10.1182/blood.V87.2.557.bloodjournal872557.

Medvinsky A, Rybtsov S, Taoudi S. Embryonic origin of the adult hematopoietic system: advances and questions. Development. 2011;138(6):1017-31. Epub 2011/02/24. doi: 10.1242/dev.040998. PubMed PMID: 21343360.

Mende N, Jolly A, Percin GI, Gunther M, Rostovskaya M, Krishnan SM, et al. Prospective isolation of nonhematopoietic cells of the niche and their differential molecular interactions with HSCs. Blood. 2019;134(15):1214-26. Epub 2019/08/02. doi: 10.1182/blood.2019000176. PubMed PMID: 31366622.

Mendez-Ferrer S, Lucas D, Battista M, Frenette PS. Haematopoietic stem cell release is regulated by circadian oscillations. Nature. 2008;452(7186):442-7. Epub 2008/02/08. doi: 10.1038/nature06685. PubMed PMID: 18256599.

Mendez-Ferrer S, Michurina TV, Ferraro F, Mazloom AR, Macarthur BD, Lira SA, et al. Mesenchymal and haematopoietic stem cells form a unique bone marrow niche. Nature. 2010;466(7308):829-34. Epub 2010/08/13. doi: 10.1038/nature09262. PubMed PMID: 20703299; PubMed Central PMCID: PMCPMC3146551. 
Miller CL, Dykstra B, Eaves CJ. Characterization of mouse hematopoietic stem and progenitor cells. Curr Protoc Immunol. 2008 Feb;Chapter 22:Unit 22B.2. doi: 10.1002/0471142735.im22b02s80. PMID: 18432636.

Moignard V, Macaulay IC, Swiers G, Buettner F, Schutte J, Calero-Nieto FJ, et al. Characterization of transcriptional networks in blood stem and progenitor cells using high-throughput single-cell gene expression analysis. Nat Cell Biol. 2013;15(4):363-72. Epub 2013/03/26. doi: 10.1038/ncb2709. PubMed PMID: 23524953; PubMed Central PMCID: PMCPMC3796878.

Moore KA, Ema H, Lemischka IR. In Vitro Maintenance of Highly Purified, Transplantable Hematopoietic Stem Cells. Blood. 1997a;89(12):4337-47. doi: 10.1182/blood.V89.12.4337.

Moore KA, Pytowski B, Witte L, Hicklin D, Lemischka IR. Hematopoietic activity of a stromal cell transmembrane protein containing epidermal growth factor-like repeat motifs. Proc Natl Acad Sci U S A. 1997b;94(8):4011-6. Epub 1997/04/15. doi: 10.1073/pnas.94.8.4011. PubMed PMID: 9108096; PubMed Central PMCID: PMCPMC20559.

Morita Y, Ema H, Nakauchi H. Heterogeneity and hierarchy within the most primitive hematopoietic stem cell compartment. J Exp Med. 2010;207(6):1173-82. Epub 2010/04/28. doi: 10.1084/jem.20091318. PubMed PMID: 20421392; PubMed Central PMCID: PMCPMC2882827.

Morita Y, Iseki A, Okamura S, Suzuki S, Nakauchi H, Ema H. Functional characterization of hematopoietic stem cells in the spleen. Exp Hematol. 2011;39(3):3519 e3. Epub 2010/12/28. doi: 10.1016/j.exphem.2010.12.008. PubMed PMID: 21185906.

Morrison SJ, Weissman IL. The long-term repopulating subset of hematopoietic stem cells is deterministic and isolatable by phenotype. Immunity. 1994;1(8):661-73. doi: 10.1016/1074-7613(94)90037-x.

Morrison SJ, Hemmati HD, Wandycz AM, Weissman IL. The purification and characterization of fetal liver hematopoietic stem cells. Proc Natl Acad Sci U S A. 1995 Oct 24;92(22):10302-6. doi: 10.1073/pnas.92.22.10302. PMID: 7479772; PMCID: PMC40784.

Morrison SJ, Wandycz AM, Hemmati HD, Wright DE, Weissman IL. Identification of a lineage of multipotent hematopoietic progenitors. Development. 1997 May;124(10):1929-39. PMID: 9169840.

Morrison SJ, Scadden DT. The bone marrow niche for haematopoietic stem cells. Nature. 2014;505(7483):327-34. Epub 2014/01/17. doi: 10.1038/nature12984. PubMed PMID: 24429631; PubMed Central PMCID: PMCPMC4514480. 
Müller J. Handbuch der Physiologie des Menschen 1844 (Coblenz: Jakob Hölscher).

Müller AM, Medvinsky A, Strouboulis J, Grosveld F, Dzierzakt E. Development of hematopoietic stem cell activity in the mouse embryo. Immunity. 1994;1(4):291-301. doi: 10.1016/1074-7613(94)90081-7.

Müller-Sieburg CE, Whitlock CA, Weissman IL. Isolation of two early B lymphocyte progenitors from mouse marrow: A committed Pre-Pre-B cell and a clonogenic Thy-1lo hematopoietic stem cell. Cell. 1986;44(4):653-62. doi: 10.1016/0092-8674(86)90274-6.

Müller-Sieburg CE, Cho RH, Thoman M, Adkins B, Sieburg HB. Deterministic regulation of hematopoietic stem cell self-renewal and differentiation. Blood. 2002;100(4):1302-9. doi: 10.1182/blood.V100.4.1302.h81602001302_1302_1309.

Müller-Sieburg CE, Cho RH, Karlsson L, Huang JF, Sieburg HB. Myeloid-biased hematopoietic stem cells have extensive self-renewal capacity but generate diminished lymphoid progeny with impaired IL-7 responsiveness. Blood. 2004;103(11):4111-8. Epub 2004/02/21. doi: 10.1182/blood-2003-10-3448. PubMed PMID: 14976059.

Na Nakorn T, Traver D, Weissman IL, Akashi K. Myeloerythroid-restricted progenitors are sufficient to confer radioprotection and provide the majority of day 8 CFU-S. Journal of Clinical Investigation. 2002;109(12):1579-85. doi: 10.1172/jci0215272.

Naito M, Yamamura F, Nishikawa S, Takahashi K. Development, differentiation, and maturation of fetal mouse yolk sac macrophages in cultures. J Leukoc Biol. 1989;46(1):110. Epub 1989/07/01. doi: 10.1002/jlb.46.1.1. PubMed PMID: 2732624.

Nakamura-Ishizu A, Takubo K, Kobayashi H, Suzuki-Inoue K, Suda T. CLEC-2 in megakaryocytes is critical for maintenance of hematopoietic stem cells in the bone marrow. J Exp Med. 2015;212(12):2133-46. Epub 2015/11/11. doi:

10.1084/jem.20150057. PubMed PMID: 26552707; PubMed Central PMCID: PMCPMC4647260.

Naveiras O, Nardi V, Wenzel PL, Hauschka PV, Fahey F, Daley GQ. Bone-marrow adipocytes as negative regulators of the haematopoietic microenvironment. Nature. 2009;460(7252):259-63. Epub 2009/06/12. doi: 10.1038/nature08099. PubMed PMID: 19516257; PubMed Central PMCID: PMCPMC2831539.

Nestorowa S, Hamey FK, Pijuan Sala B, Diamanti E, Shepherd M, Laurenti E, et al. A single-cell resolution map of mouse hematopoietic stem and progenitor cell differentiation. Blood. 2016;128(8):e20-31. Epub 2016/07/02. doi: 10.1182/blood-201605-716480. PubMed PMID: 27365425; PubMed Central PMCID: PMCPMC5305050.

Neumann E. Zentralbl. f. d. mediz. Wiss. 1868; (44):689. 
Neumann E. Arch. f. Mikrosk. Anatomie und Entwicklungsgeschichte 1912;(207):480520.

Nolta JA, Thiemann FT, Arakawa-Hoyt J, Dao MA, Barsky LW, Moore KA, et al. The AFT024 stromal cell line supports long-term ex vivo maintenance of engrafting multipotent human hematopoietic progenitors. Leukemia. 2002 Mar;16(3):352-61. doi: 10.1038/sj.leu.2402371. PMID: 11896538.

North T, Gu TL, Stacy T, Wang Q, Howard L, Binder M, et al. Cbfa2 is required for the formation of intra-aortic hematopoietic clusters. Development. 1999 Jun;126(11):256375. PMID: 10226014.

North TE, de Bruijn MFTR, Stacy T, Talebian L, Lind E, Robin C, et al. Runx1 Expression Marks Long-Term Repopulating Hematopoietic Stem Cells in the Midgestation Mouse Embryo. Immunity. 2002;16(5):661-72. doi: 10.1016/s10747613(02)00296-0.

North TE, Goessling W, Walkley CR, Lengerke C, Kopani KR, Lord AM, et al. Prostaglandin E2 regulates vertebrate haematopoietic stem cell homeostasis. Nature. 2007;447(7147):1007-11. Epub 2007/06/22. doi: 10.1038/nature05883. PubMed PMID: 17581586; PubMed Central PMCID: PMCPMC2775137.

Nowell PC, Cole LJ, Habermeyer JG, Roan PL. Growth and continued function of rat marrow cells in x-radiated mice. Cancer Res. 1956 Mar;16(3):258-61. PMID: 13304871.

Oguro H, Ding L, Morrison SJ. SLAM family markers resolve functionally distinct subpopulations of hematopoietic stem cells and multipotent progenitors. Cell Stem Cell. 2013;13(1):102-16. Epub 2013/07/06. doi: 10.1016/j.stem.2013.05.014. PubMed PMID: 23827712; PubMed Central PMCID: PMCPMC3736853.

Okuda T, van Deursen J, Hiebert SW, Grosveld G, Downing JR. AML1, the Target of Multiple Chromosomal Translocations in Human Leukemia, Is Essential for Normal Fetal Liver Hematopoiesis. Cell. 1996;84(2):321-30. doi: 10.1016/s0092-8674(00)809861.

Olsen BR, Reginato AM, Wang W. Bone development. Annu Rev Cell Dev Biol. 2000;16:191-220. Epub 2000/10/14. doi: 10.1146/annurev.cellbio.16.1.191. PubMed PMID: 11031235.

Osawa M, Hanada K, Hamada H, Nakauchi H. Long-term lymphohematopoietic reconstitution by a single CD34-low/negative hematopoietic stem cell. Science. 1996;273(5272):242-5. Epub 1996/07/12. doi: 10.1126/science.273.5272.242. PubMed PMID: 8662508. 
Ottersbach K, Dzierzak E. The murine placenta contains hematopoietic stem cells within the vascular labyrinth region. Dev Cell. 2005;8(3):377-87. Epub 2005/03/02. doi: 10.1016/j.devcel.2005.02.001. PubMed PMID: 15737933.

Ovchinnikov DA, van Zuylen WJ, DeBats CE, Alexander KA, Kellie S, Hume DA. Expression of Gal4-dependent transgenes in cells of the mononuclear phagocyte system labeled with enhanced cyan fluorescent protein using Csf1r-Gal4VP16/UAS-ECFP double-transgenic mice. J Leukoc Biol. 2008;83(2):430-3. Epub 2007/11/01. doi: 10.1189/jlb.0807585. PubMed PMID: 17971498.

Owen RD. Immunogenetic Consequences of Vascular Anastomoses between Bovine Twins. Science. 1945;102(2651):400-1. Epub 1945/10/19. doi:

10.1126/science.102.2651.400. PubMed PMID: 17755278.

Palis J, Chan RJ, Koniski A, Patel R, Starr M, Yoder MC. Spatial and temporal emergence of high proliferative potential hematopoietic precursors during murine embryogenesis. Proc Natl Acad Sci U S A. 2001;98(8):4528-33. Epub 2001/04/11. doi: 10.1073/pnas.071002398. PubMed PMID: 11296291; PubMed Central PMCID: PMCPMC31868.

Pappenheim A. Virchows Arch. 1896;(145):587-643.

Pappenheim A. Fol. Haematol. 1908;(6):217-242.

Paul F, Arkin Y, Giladi A, Jaitin DA, Kenigsberg E, Keren-Shaul H, et al. Transcriptional Heterogeneity and Lineage Commitment in Myeloid Progenitors. Cell. 2015;163(7):1663-77. Epub 2015/12/03. doi: 10.1016/j.cell.2015.11.013. PubMed PMID: 26627738.

Pellin D, Loperfido M, Baricordi C, Wolock SL, Montepeloso A, Weinberg OK, et al. A comprehensive single cell transcriptional landscape of human hematopoietic progenitors. Nat Commun. 2019;10(1):2395. Epub 2019/06/05. doi: 10.1038/s41467-019-10291-0. PubMed PMID: 31160568; PubMed Central PMCID: PMCPMC6546699.

Pereira CF, Chang B, Gomes A, Bernitz J, Papatsenko D, Niu X, et al. Hematopoietic Reprogramming In Vitro Informs In Vivo Identification of Hemogenic Precursors to Definitive Hematopoietic Stem Cells. Dev Cell. 2016;36(5):525-39. Epub 2016/03/10. doi: 10.1016/j.devcel.2016.02.011. PubMed PMID: 26954547; PubMed Central PMCID: PMCPMC4785845.

Pietras EM, Reynaud D, Kang YA, Carlin D, Calero-Nieto FJ, Leavitt AD, et al. Functionally Distinct Subsets of Lineage-Biased Multipotent Progenitors Control Blood Production in Normal and Regenerative Conditions. Cell Stem Cell. 2015;17(1):35-46. Epub 2015/06/23. doi: 10.1016/j.stem.2015.05.003. PubMed PMID: 26095048; PubMed Central PMCID: PMCPMC4542150. 
Pietras EM. Inflammation: a key regulator of hematopoietic stem cell fate in health and disease. Blood. 2017;130(15):1693-8. Epub 2017/09/07. doi: 10.1182/blood-2017-06780882. PubMed PMID: 28874349; PubMed Central PMCID: PMCPMC5639485.

Ploemacher RE, van der Sluijs JP, Voerman JS, Brons NH. An in vitro limiting-dilution assay of long-term repopulating hematopoietic stem cells in the mouse. Blood.

1989;74(8):2755-63. doi: 10.1182/blood.V74.8.2755.2755.

Pluznik DH, Sachs L. The cloning of normal "mast" cells in tissue culture. J Cell Physiol. 1965;66(3):319-24. Epub 1965/12/01. doi: 10.1002/jcp.1030660309. PubMed PMID: 5884359.

Porter RL, Georger MA, Bromberg O, McGrath KE, Frisch BJ, Becker MW, et al. Prostaglandin E2 increases hematopoietic stem cell survival and accelerates hematopoietic recovery after radiation injury. Stem Cells. 2013;31(2):372-83. Epub 2012/11/22. doi: 10.1002/stem.1286. PubMed PMID: 23169593; PubMed Central PMCID: PMCPMC3580384.

Potts KS, Sargeant TJ, Markham JF, Shi W, Biben C, Josefsson EC, et al. A lineage of diploid platelet-forming cells precedes polyploid megakaryocyte formation in the mouse embryo. Blood. 2014;124(17):2725-9. Epub 2014/08/01. doi: 10.1182/blood-2014-02559468. PubMed PMID: 25079356.

Potts KS, Sargeant TJ, Dawson CA, Josefsson EC, Hilton DJ, Alexander WS, et al. Mouse prenatal platelet-forming lineages share a core transcriptional program but divergent dependence on MPL. Blood. 2015;126(6):807-16. Epub 2015/05/23. doi: 10.1182/blood-2014-12-616607. PubMed PMID: 25995324.

Ratajczak CK, Muglia LJ. Insights into parturition biology from genetically altered mice. Pediatr Res. 2008;64(6):581-9. Epub 2008/08/06. doi: 10.1203/PDR.0b013e31818718d2. PubMed PMID: 18679156.

Raynaud CM, Butler JM, Halabi NM, Ahmad FS, Ahmed B, Rafii S, et al. Endothelial cells provide a niche for placental hematopoietic stem/progenitor cell expansion through broad transcriptomic modification. Stem Cell Res. 2013;11(3):1074-90. Epub 2013/08/28. doi: 10.1016/j.scr.2013.07.010. PubMed PMID: 23978474.

Rhodes KE, Gekas C, Wang Y, Lux CT, Francis CS, Chan DN, et al. The emergence of hematopoietic stem cells is initiated in the placental vasculature in the absence of circulation. Cell Stem Cell. 2008;2(3):252-63. Epub 2008/03/29. doi: 10.1016/j.stem.2008.01.001. PubMed PMID: 18371450; PubMed Central PMCID: PMCPMC2888040.

Robert-Moreno A, Espinosa L, de la Pompa JL, Bigas A. RBPjkappa-dependent Notch function regulates Gata2 and is essential for the formation of intra-embryonic 
hematopoietic cells. Development. 2005;132(5):1117-26. Epub 2005/02/04. doi: 10.1242/dev.01660. PubMed PMID: 15689374.

Rodewald HR, Dessing M, Dvorak AM, Galli SJ. Identification of a committed precursor for the mast cell lineage. Science. 1996;271(5250):818-22. Epub 1996/02/09. doi: 10.1126/science.271.5250.818. PubMed PMID: 8629001.

Rodriguez-Fraticelli AE, Wolock SL, Weinreb CS, Panero R, Patel SH, Jankovic M, et al. Clonal analysis of lineage fate in native haematopoiesis. Nature. 2018;553(7687):2126. Epub 2018/01/13. doi: 10.1038/nature25168. PubMed PMID: 29323290; PubMed Central PMCID: PMCPMC5884107.

Rowlinson JM, Gering M. Hey2 acts upstream of Notch in hematopoietic stem cell specification in zebrafish embryos. Blood. 2010;116(12):2046-56. Epub 2010/06/01. doi: 10.1182/blood-2009-11-252635. PubMed PMID: 20511544.

Rybtsov S, Sobiesiak M, Taoudi S, Souilhol C, Senserrich J, Liakhovitskaia A, et al. Hierarchical organization and early hematopoietic specification of the developing HSC lineage in the AGM region. J Exp Med. 2011;208(6):1305-15. Epub 2011/06/01. doi: 10.1084/jem.20102419. PubMed PMID: 21624936; PubMed Central PMCID: PMCPMC3173253.

Rybtsov S, Batsivari A, Bilotkach K, Paruzina D, Senserrich J, Nerushev O, et al. Tracing the origin of the HSC hierarchy reveals an SCF-dependent, IL-3-independent CD43(-) embryonic precursor. Stem Cell Reports. 2014;3(3):489-501. Epub 2014/09/23. doi: 10.1016/j.stemcr.2014.07.009. PubMed PMID: 25241746; PubMed Central PMCID: PMCPMC4266012.

Sabin FR. Studies on the origin of blood vessels and of red blood corpuscules as seen in the living blastoderm of chicks during the second day of incubation. Contrib. Embryol. 1920;(9):213-262.

Sandler VM, Lis R, Liu Y, Kedem A, James D, Elemento O, et al. Reprogramming human endothelial cells to haematopoietic cells requires vascular induction. Nature. 2014;511(7509):312-8. Epub 2014/07/18. doi: 10.1038/nature13547. PubMed PMID: 25030167; PubMed Central PMCID: PMCPMC4159670.

Sanjuan-Pla A, Macaulay IC, Jensen CT, Woll PS, Luis TC, Mead A, et al. Plateletbiased stem cells reside at the apex of the haematopoietic stem-cell hierarchy. Nature. 2013;502(7470):232-6. Epub 2013/08/13. doi: 10.1038/nature12495. PubMed PMID: 23934107.

Sasaki T, Mizuochi C, Horio Y, Nakao K, Akashi K, Sugiyama D. Regulation of hematopoietic cell clusters in the placental niche through $\mathrm{SCF} / \mathrm{Kit}$ signaling in embryonic mouse. Development. 2010;137(23):3941-52. Epub 2010/10/29. doi:

10.1242/dev.051359. PubMed PMID: 20980401. 
Sawai CM, Babovic S, Upadhaya S, Knapp D, Lavin Y, Lau CM, et al. Hematopoietic Stem Cells Are the Major Source of Multilineage Hematopoiesis in Adult Animals. Immunity. 2016;45(3):597-609. Epub 2016/09/04. doi: 10.1016/j.immuni.2016.08.007. PubMed PMID: 27590115; PubMed Central PMCID: PMCPMC5054720.

Sawen P, Eldeeb M, Erlandsson E, Kristiansen TA, Laterza C, Kokaia Z, et al. Murine HSCs contribute actively to native hematopoiesis but with reduced differentiation capacity upon aging. Elife. 2018;7. Epub 2018/12/19. doi: 10.7554/eLife.41258. PubMed PMID: 30561324; PubMed Central PMCID: PMCPMC6298771.

Schaefer BC, Schaefer ML, Kappler JW, Marrack P, Kedl RM. Observation of antigendependent CD8+ T-cell/ dendritic cell interactions in vivo. Cell Immunol.

2001;214(2):110-22. Epub 2002/06/29. doi: 10.1006/cimm.2001.1895. PubMed PMID: 12088410.

Schofield R. The relationship between the spleen colony-forming cell and the haemopoietic stem cell. Blood Cells. 1978;4(1-2):7-25. PMID: 747780.

Senn JS, McCulloch EA, Till JE. Comparison of Colony-Forming Ability of Normal and LeukÆmic Human Marrow in Cell Culture. The Lancet. 1967;290(7516):597-8. doi: 10.1016/s0140-6736(67)90742-8.

Severe N, Karabacak NM, Gustafsson K, Baryawno N, Courties G, Kfoury Y, et al. Stress-Induced Changes in Bone Marrow Stromal Cell Populations Revealed through Single-Cell Protein Expression Mapping. Cell Stem Cell. 2019;25(4):570-83 e7. Epub 2019/07/08. doi: 10.1016/j.stem.2019.06.003. PubMed PMID: 31279774; PubMed Central PMCID: PMCPMC6778015.

Seyda M, Elkhal A, Quante M, Falk CS, Tullius SG. T Cells Going Innate. Trends Immunol. 2016;37(8):546-56. Epub 2016/07/13. doi: 10.1016/j.it.2016.06.004. PubMed PMID: 27402226; PubMed Central PMCID: PMCPMC5633931.

Shalaby F, Rossant J, Yamaguchi TP, Gertsenstein M, Wu XF, Breitman ML, et al. Failure of blood-island formation and vasculogenesis in Flk-1-deficient mice. Nature. 1995;376(6535):62-6. Epub 1995/07/06. doi: 10.1038/376062a0. PubMed PMID: 7596435 .

Shen FW, Saga Y, Litman G, Freeman G, Tung JS, Cantor H, et al. Cloning of Ly-5 cDNA. Proc Natl Acad Sci U S A. 1985;82(21):7360-3. Epub 1985/11/01. doi: 10.1073/pnas.82.21.7360. PubMed PMID: 3864163; PubMed Central PMCID: PMCPMC391344.

Shen FW, Tung JS, Boyse EA. Further definition of the Ly-5 system. Immunogenetics. 1986;24(3):146-9. Epub 1986/01/01. doi: 10.1007/BF00364741. PubMed PMID: 3489673. 
Silberstein L, Goncalves KA, Kharchenko PV, Turcotte R, Kfoury Y, Mercier F, et al. Proximity-Based Differential Single-Cell Analysis of the Niche to Identify Stem/Progenitor Cell Regulators. Cell Stem Cell. 2016;19(4):530-43. Epub 2016/08/16. doi: 10.1016/j.stem.2016.07.004. PubMed PMID: 27524439; PubMed Central PMCID: PMCPMC5402355.

Siminovitch L, McCulloch EA, Till JE. The Distribution of Colony-Forming Cells among Spleen Colonies. J Cell Comp Physiol. 1963;62:327-36. Epub 1963/12/01. doi: 10.1002/jcp.1030620313. PubMed PMID: 14086156.

Smith LG, Weissman IL, Heimfeld S. Clonal analysis of hematopoietic stem-cell differentiation in vivo. Proc Natl Acad Sci U S A. 1991;88(7):2788-92. Epub 1991/04/01. doi: 10.1073/pnas.88.7.2788. PubMed PMID: 1672767; PubMed Central PMCID: PMCPMC51324.

Solaimani Kartalaei P, Yamada-Inagawa T, Vink CS, de Pater E, van der Linden R, Marks-Bluth J, et al. Whole-transcriptome analysis of endothelial to hematopoietic stem cell transition reveals a requirement for Gpr56 in HSC generation. J Exp Med. 2015;212(1):93-106. Epub 2014/12/31. doi: 10.1084/jem.20140767. PubMed PMID: 25547674; PubMed Central PMCID: PMCPMC4291529.

Sonoda T, Hayashi C, Kitamura Y. Presence of mast cell precursors in the yolk sac of mice. Developmental Biology. 1983;97(1):89-94. doi: 10.1016/0012-1606(83)90066-0.

Spangrude GJ, Heimfeld S, Weissman IL. Purification and characterization of mouse hematopoietic stem cells. Science. 1988;241(4861):58-62. Epub 1988/07/01. doi: 10.1126/science.2898810. PubMed PMID: 2898810.

Spindler TJ, Tseng AW, Zhou X, Adams GB. Adipocytic cells augment the support of primitive hematopoietic cells in vitro but have no effect in the bone marrow niche under homeostatic conditions. Stem Cells Dev. 2014;23(4):434-41. Epub 2013/10/03. doi: 10.1089/scd.2013.0227. PubMed PMID: 24083324; PubMed Central PMCID: PMCPMC3996940.

St John AL, Abraham SN. Innate immunity and its regulation by mast cells. J Immunol. 2013;190(9):4458-63. Epub 2013/04/23. doi: 10.4049/jimmunol.1203420. PubMed PMID: 23606723; PubMed Central PMCID: PMCPMC3645001.

Stoeckius M, Hafemeister C, Stephenson W, Houck-Loomis B, Chattopadhyay PK, Swerdlow H, et al. Simultaneous epitope and transcriptome measurement in single cells. Nat Methods. 2017;14(9):865-8. Epub 2017/08/02. doi: 10.1038/nmeth.4380. PubMed PMID: 28759029; PubMed Central PMCID: PMCPMC5669064.

Sugimoto Y, Yamasaki A, Segi E, Tsuboi K, Aze Y, Nishimura T, et al. Failure of parturition in mice lacking the prostaglandin F receptor. Science. 1997;277(5326):681-3. Epub 1997/08/01. doi: 10.1126/science.277.5326.681. PubMed PMID: 9235889. 
Sun J, Ramos A, Chapman B, Johnnidis JB, Le L, Ho YJ, et al. Clonal dynamics of native haematopoiesis. Nature. 2014;514(7522):322-7. Epub 2014/10/09. doi:

10.1038/nature13824. PubMed PMID: 25296256; PubMed Central PMCID:

PMCPMC4408613.

Swiers G, Baumann C, O'Rourke J, Giannoulatou E, Taylor S, Joshi A, et al. Early dynamic fate changes in haemogenic endothelium characterized at the single-cell level. Nat Commun. 2013;4:2924. Epub 2013/12/12. doi: 10.1038/ncomms3924. PubMed PMID: 24326267; PubMed Central PMCID: PMCPMC4362711.

Taichman RS, Emerson SG. Human osteoblasts support hematopoiesis through the production of granulocyte colony-stimulating factor. J Exp Med. 1994;179(5):1677-82. Epub 1994/05/01. doi: 10.1084/jem.179.5.1677. PubMed PMID: 7513014; PubMed Central PMCID: PMCPMC2191506.

Taichman RS, Reilly MJ, Emerson SG. Human osteoblasts support human hematopoietic progenitor cells in vitro bone marrow cultures. Blood. 1996;87(2):518-24. doi:

10.1182/blood.V87.2.518.bloodjournal872518.

Takahashi K, Yamamura F, Naito M. Differentiation, maturation, and proliferation of macrophages in the mouse yolk sac: a light-microscopic, enzyme-cytochemical, immunohistochemical, and ultrastructural study. J Leukoc Biol. 1989;45(2):87-96. Epub 1989/02/01. doi: 10.1002/jlb.45.2.87. PubMed PMID: 2536795.

Taoudi S, Morrison AM, Inoue H, Gribi R, Ure J, Medvinsky A. Progressive divergence of definitive haematopoietic stem cells from the endothelial compartment does not depend on contact with the foetal liver. Development. 2005;132(18):4179-91. Epub 2005/08/19. doi: 10.1242/dev.01974. PubMed PMID: 16107475.

Tavian M, Coulombel L, Luton D, Clemente HS, Dieterlen-Lievre F, Peault B. Aortaassociated CD34+ hematopoietic cells in the early human embryo. Blood. 1996;87(1):6772. doi: 10.1182/blood.V87.1.67.67.

Thiemann FT, Moore KA, Smogorzewska EM, Lemischka IR, Crooks GM. The murine stromal cell line AFT024 acts specifically on human CD34+CD38- progenitors to maintain primitive function and immunophenotype in vitro. Exp Hematol. 1998 Jul;26(7):612-9. PMID: 9657136.

Thomas ED, Lochte HL, Jr., Lu WC, Ferrebee JW. Intravenous infusion of bone marrow in patients receiving radiation and chemotherapy. N Engl J Med. 1957;257(11):491-6. Epub 1957/09/12. doi: 10.1056/NEJM195709122571102. PubMed PMID: 13464965.

Tikhonova AN, Dolgalev I, Hu H, Sivaraj KK, Hoxha E, Cuesta-Dominguez A, et al. The bone marrow microenvironment at single-cell resolution. Nature. 2019;569(7755):222-8. Epub 2019/04/12. doi: 10.1038/s41586-019-1104-8. PubMed PMID: 30971824; PubMed Central PMCID: PMCPMC6607432. 
Till JE, McCulloch EA. A direct measurement of the radiation sensitivity of normal mouse bone marrow cells. 1961. Radiat Res. 2012;178(2):AV3-7. Epub 2012/08/09. doi: 10.1667/rrav01.1. PubMed PMID: 22870977.

Tober J, Koniski A, McGrath KE, Vemishetti R, Emerson R, de Mesy-Bentley KK, et al. The megakaryocyte lineage originates from hemangioblast precursors and is an integral component both of primitive and of definitive hematopoiesis. Blood. 2007;109(4):143341. Epub 2006/10/26. doi: 10.1182/blood-2006-06-031898. PubMed PMID: 17062726; PubMed Central PMCID: PMCPMC1794060.

Trentin JJ. Mortality and skin transplantability in X-irradiated mice receiving isologous, homologous or heterologous bone marrow. Proc Soc Exp Biol Med. 1956;92(4):688-93. Epub 1956/08/01. doi: 10.3181/00379727-92-22582. PubMed PMID: 13370496.

Tusi BK, Wolock SL, Weinreb C, Hwang Y, Hidalgo D, Zilionis R, et al. Population snapshots predict early haematopoietic and erythroid hierarchies. Nature.

2018;555(7694):54-60. Epub 2018/02/22. doi: 10.1038/nature25741. PubMed PMID: 29466336; PubMed Central PMCID: PMCPMC5899604.

Uchida N, Aguila HL, Fleming WH, Jerabek L, Weissman IL. Rapid and sustained hematopoietic recovery in lethally irradiated mice transplanted with purified Thy-1.11o Lin-Sca-1+ hematopoietic stem cells. Blood. 1994 Jun 15;83(12):3758-79. PMID: 7911343.

Uchida N, Dykstra B, Lyons KJ, Leung FY, Eaves CJ. Different in vivo repopulating activities of purified hematopoietic stem cells before and after being stimulated to divide in vitro with the same kinetics. Exp Hematol. 2003;31(12):1338-47. Epub 2003/12/10. doi: 10.1016/j.exphem.2003.09.001. PubMed PMID: 14662343.

Ueno H, Weissman IL. Clonal analysis of mouse development reveals a polyclonal origin for yolk sac blood islands. Dev Cell. 2006;11(4):519-33. Epub 2006/10/03. doi: 10.1016/j.devcel.2006.08.001. PubMed PMID: 17011491.

Van Laere AS, Nguyen M, Braunschweig M, Nezer C, Collette C, Moreau L, et al. A regulatory mutation in IGF2 causes a major QTL effect on muscle growth in the pig. Nature. 2003;425(6960):832-6. Epub 2003/10/24. doi: 10.1038/nature02064. PubMed PMID: 14574411.

Velten L, Haas SF, Raffel S, Blaszkiewicz S, Islam S, Hennig BP, et al. Human haematopoietic stem cell lineage commitment is a continuous process. Nat Cell Biol. 2017;19(4):271-81. Epub 2017/03/21. doi: 10.1038/ncb3493. PubMed PMID: 28319093; PubMed Central PMCID: PMCPMC5496982.

Watanabe Y, Aiba Y, Katsura Y. T cell progenitors in the murine fetal liver: differences from those in the adult bone marrow. Cell Immunol. 1997;177(1):18-25. Epub 1997/04/10. doi: 10.1006/cimm.1997.1094. PubMed PMID: 9140092. 
Weismann A. Die Continuität des Keimplasmas als Grundlage einer Theorie der Vererbung 1885 (Jena: Gustav Fischer).

Weissman IL, Shizuru JA. The origins of the identification and isolation of hematopoietic stem cells, and their capability to induce donor-specific transplantation tolerance and treat autoimmune diseases. Blood. 2008;112(9):3543-53. Epub 2008/10/25. doi:

10.1182/blood-2008-08-078220. PubMed PMID: 18948588; PubMed Central PMCID: PMCPMC2574516.

Whitlock CA, Witte ON. Long-term culture of B lymphocytes and their precursors from murine bone marrow. Proc Natl Acad Sci U S A. 1982;79(11):3608-12. Epub 1982/06/01. doi: 10.1073/pnas.79.11.3608. PubMed PMID: 6980414; PubMed Central PMCID: PMCPMC346472.

Whitlock CA, Tidmarsh GF, Muller-Sieburg C, Weissman IL. Bone marrow stromal cell lines with lymphopoietic activity express high levels of a pre-B neoplasia-associated molecule. Cell. 1987;48(6):1009-21. doi: 10.1016/0092-8674(87)90709-4.

Wilson A, Laurenti E, Oser G, van der Wath RC, Blanco-Bose W, Jaworski M, et al. Hematopoietic stem cells reversibly switch from dormancy to self-renewal during homeostasis and repair. Cell. 2008;135(6):1118-29. Epub 2008/12/09. doi: 10.1016/j.cell.2008.10.048. PubMed PMID: 19062086.

Wilson EB. The Cell in Development and Inheritance 1896 (New York: Macmillan).

Wilson NK, Kent DG, Buettner F, Shehata M, Macaulay IC, Calero-Nieto FJ, et al. Combined Single-Cell Functional and Gene Expression Analysis Resolves Heterogeneity within Stem Cell Populations. Cell Stem Cell. 2015;16(6):712-24. Epub 2015/05/26. doi: 10.1016/j.stem.2015.04.004. PubMed PMID: 26004780; PubMed Central PMCID: PMCPMC4460190.

Wineman J, Moore K, Lemischka I, Muller-Sieburg C. Functional heterogeneity of the hematopoietic microenvironment: rare stromal elements maintain long-term repopulating stem cells. Blood. 1996;87(10):4082-90. doi:

10.1182/blood.V87.10.4082.bloodjournal87104082.

Winkler IG, Sims NA, Pettit AR, Barbier V, Nowlan B, Helwani F, et al. Bone marrow macrophages maintain hematopoietic stem cell (HSC) niches and their depletion mobilizes HSCs. Blood. 2010;116(23):4815-28. Epub 2010/08/18. doi: 10.1182/blood2009-11-253534. PubMed PMID: 20713966.

Wolber FM, Leonard E, Michael S, Orschell-Traycoff CM, Yoder MC, Srour EF. Roles of spleen and liver in development of the murine hematopoietic system. Experimental Hematology. 2002;30(9):1010-9. doi: 10.1016/s0301-472x(02)00881-0. 
Wolf NS, Koné A, Priestley GV, Bartelmez SH. In vivo and in vitro characterization of long-term repopulating primitive hematopoietic cells isolated by sequential Hoechst 33342-rhodamine 123 FACS selection. Exp Hematol. 1993 May;21(5):614-22. PMID: 8513861.

Wolock SL, Krishnan I, Tenen DE, Matkins V, Camacho V, Patel S, et al. Mapping Distinct Bone Marrow Niche Populations and Their Differentiation Paths. Cell Rep. 2019;28(2):302-11 e5. Epub 2019/07/11. doi: 10.1016/j.celrep.2019.06.031. PubMed PMID: 31291568 ; PubMed Central PMCID: PMCPMC6684313.

Wong PM, Chung SW, White JS, Reicheld SM, Patterson M, Clarke BJ, et al. Adult hemoglobins are synthesized in murine fetal hepatic erythropoietic cells. Blood. 1983;62(6):1280-8. doi: 10.1182/blood.V62.6.1280.1280.

Worton RG, McCulloch EA, Till JE. Physical separation of hemopoietic stem cells from cells forming colonies in culture. J Cell Physiol. 1969;74(2):171-82. Epub 1969/10/01. doi: 10.1002/jcp.1040740209. PubMed PMID: 4902120.

Wu AM, Till JE, Siminovitch L, McCulloch EA. A cytological study of the capacity for differentiation of normal hemopoietic colony-forming cells. J Cell Physiol.

1967;69(2):177-84. Epub 1967/04/01. doi: 10.1002/jcp.1040690208. PubMed PMID: 6033948.

Wu AM, Siminovitch L, Till JE, McCulloch EA. Evidence for a relationship between mouse hemopoietic stem cells and cells forming colonies in culture. Proc Natl Acad Sci U S A. 1968;59(4):1209-15. Epub 1968/04/01. doi: 10.1073/pnas.59.4.1209. PubMed PMID: 5240024; PubMed Central PMCID: PMCPMC224853.

Xu C, Gao X, Wei Q, Nakahara F, Zimmerman SE, Mar J, et al. Stem cell factor is selectively secreted by arterial endothelial cells in bone marrow. Nat Commun. 2018;9(1):2449. Epub 2018/06/24. doi: 10.1038/s41467-018-04726-3. PubMed PMID: 29934585; PubMed Central PMCID: PMCPMC6015052.

Xu MJ, Matsuoka S, Yang FC, Ebihara Y, Manabe A, Tanaka R, et al. Evidence for the presence of murine primitive megakaryocytopoiesis in the early yolk sac. Blood. 2001;97(7):2016-22. Epub 2001/03/27. doi: 10.1182/blood.v97.7.2016. PubMed PMID: 11264166.

Yamamoto R, Morita Y, Ooehara J, Hamanaka S, Onodera M, Rudolph KL, et al. Clonal analysis unveils self-renewing lineage-restricted progenitors generated directly from hematopoietic stem cells. Cell. 2013;154(5):1112-26. Epub 2013/09/03. doi: 10.1016/j.cell.2013.08.007. PubMed PMID: 23993099.

Yamazaki S, Ema H, Karlsson G, Yamaguchi T, Miyoshi H, Shioda S, et al. Nonmyelinating Schwann cells maintain hematopoietic stem cell hibernation in the bone 
marrow niche. Cell. 2011;147(5):1146-58. Epub 2011/11/29. doi:

10.1016/j.cell.2011.09.053. PubMed PMID: 22118468.

Yokomizo T, Dzierzak E. Three-dimensional cartography of hematopoietic clusters in the vasculature of whole mouse embryos. Development. 2010;137(21):3651-61. Epub 2010/09/30. doi: 10.1242/dev.051094. PubMed PMID: 20876651; PubMed Central PMCID: PMCPMC2964097.

Yokomizo T, Watanabe N, Umemoto T, Matsuo J, Harai R, Kihara Y, et al. Hlf marks the developmental pathway for hematopoietic stem cells but not for erythro-myeloid progenitors. J Exp Med. 2019;216(7):1599-614. Epub 2019/05/12. doi:

10.1084/jem.20181399. PubMed PMID: 31076455; PubMed Central PMCID: PMCPMC6605751.

Yoshimoto M, Montecino-Rodriguez E, Ferkowicz MJ, Porayette P, Shelley WC, Conway SJ, et al. Embryonic day 9 yolk sac and intra-embryonic hemogenic endothelium independently generate a B-1 and marginal zone progenitor lacking B-2 potential. Proc Natl Acad Sci U S A. 2011;108(4):1468-73. Epub 2011/01/07. doi:

10.1073/pnas.1015841108. PubMed PMID: 21209332; PubMed Central PMCID: PMCPMC3029764.

Yoshimoto M, Porayette P, Glosson NL, Conway SJ, Carlesso N, Cardoso AA, et al. Autonomous murine T-cell progenitor production in the extra-embryonic yolk sac before HSC emergence. Blood. 2012;119(24):5706-14. Epub 2012/03/21. doi: 10.1182/blood2011-12-397489. PubMed PMID: 22431573; PubMed Central PMCID: PMCPMC3382930.

Zhang J, Niu C, Ye L, Huang H, He X, Tong WG, et al. Identification of the haematopoietic stem cell niche and control of the niche size. Nature. 2003;425(6960):836-41. Epub 2003/10/24. doi: 10.1038/nature02041. PubMed PMID: 14574412 .

Zhang CC, Lodish HF. Insulin-like growth factor 2 expressed in a novel fetal liver cell population is a growth factor for hematopoietic stem cells. Blood. 2004;103(7):2513-21. Epub 2003/11/01. doi: 10.1182/blood-2003-08-2955. PubMed PMID: 14592820.

Zhao M, Perry JM, Marshall H, Venkatraman A, Qian P, He XC, et al. Megakaryocytes maintain homeostatic quiescence and promote post-injury regeneration of hematopoietic stem cells. Nat Med. 2014;20(11):1321-6. Epub 2014/10/20. doi: 10.1038/nm.3706. PubMed PMID: 25326798.

Zhao Y, Zhou J, Liu D, Dong F, Cheng H, Wang W, et al. ATF4 plays a pivotal role in the development of functional hematopoietic stem cells in mouse fetal liver. Blood. 2015;126(21):2383-91. Epub 2015/09/19. doi: 10.1182/blood-2015-03-633354. PubMed PMID: 26384355; PubMed Central PMCID: PMCPMC4653766. 
Zheng S, Papalexi E, Butler A, Stephenson W, Satija R. Molecular transitions in early progenitors during human cord blood hematopoiesis. Mol Syst Biol. 2018;14(3):e8041. Epub 2018/03/17. doi: 10.15252/msb.20178041. PubMed PMID: 29545397; PubMed Central PMCID: PMCPMC5852373.

Zoja L. Fol. Clin. Chim. Micr. 1910;(2):370-372

Zovein AC, Hofmann JJ, Lynch M, French WJ, Turlo KA, Yang Y, et al. Fate tracing reveals the endothelial origin of hematopoietic stem cells. Cell Stem Cell. 2008;3(6):62536. Epub 2008/12/02. doi: 10.1016/j.stem.2008.09.018. PubMed PMID: 19041779; PubMed Central PMCID: PMCPMC2631552. 


\section{VITA}

Trent Hall was born in Marion, Arkansas in 1989. After graduating from Marion High School, he attended the University of Central Arkansas for his B.S. (2011) and M.S. (2013) in Biology. Trent then spent two years as a research technician in the lab of Shannon McKinney-Freeman at St. Jude Children's Research Hospital before joining the McKinney-Freeman lab as a University of Tennessee Health Science Center graduate student in 2015. Trent will receive his PhD in Biomedical Sciences from UTHSC in September 2020. 